\title{
MONITORING THE MIGRATIONS OF WILD SNAKE RIVER SPRING/SUMMER CHINOOK SALMON SMOLTS, 1995
}

\author{
Prepared by: \\ Stephen Achord \\ M. Brad Eppard \\ Benjamin P. Sandford \\ and \\ Gene M. Matthews
}

Funded by:

Coastal Zone and Estuarine Studies Division

Northwest Fisheries Science Center

National Marine Fisheries Service

National Oceanic and Atmospheric

and

U. S. Department of Energy

Bonneville Power Administration

Environment, Fish and Wildlife

P-0. Box 3621

Portland, OR 97208-362 1

Project Number 9 1-028

Contract Number DE-A179-91BP18800

September 1996 


\section{CONTENTS}

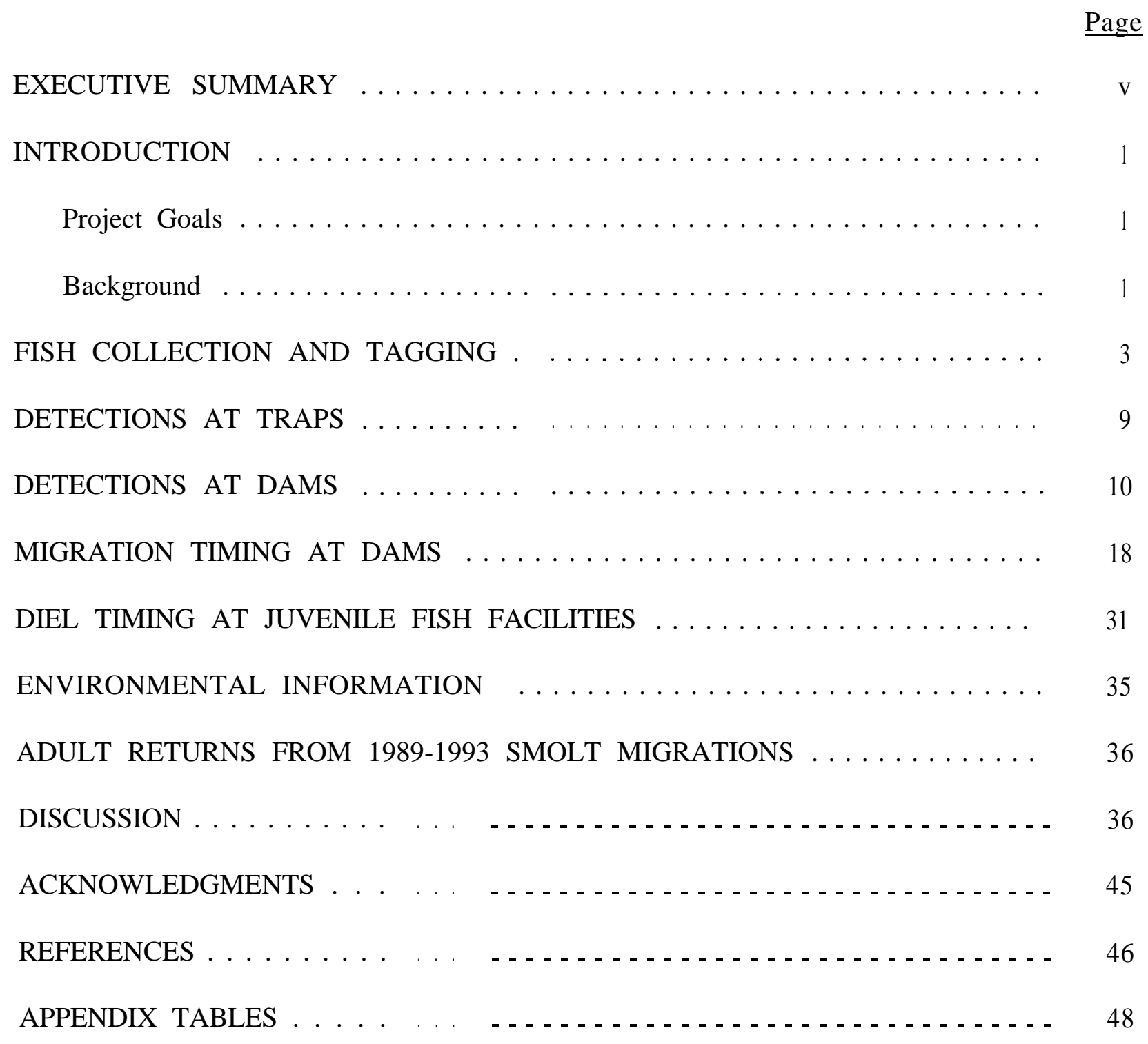




\section{EXECUTIVE SUMMARY}

We PIT tagged wild spring/summer chinook salmon parr in the Snake River Basin in 1994 and subsequently monitored these fish during their smolt migration through Lower Granite, Little Goose. Lower Monumental. McNary. John Day. and Bonneville Dams during spring. summer. and fall 1995. This report details our findings, which are summarized below.

1) We PIT tagged and released 18.459 wild chinook salmon parr in 17 streams in Idaho in July and August 1994.

2) The average overall observed mortality from collection, tagging. and 24-hour holding was $1.5 \%$. No PIT tags were lost during the 24-hour holding period to assess delayed mortality from collection. handling. and tagging.

3) In 1995. the overall adjusted percentage of PIT-tagged fish released and subsequently detected at the sis dams averaged $11.8 \%$ (range 6.3 to $28.9 \%$. depending on stream of origin).

4) Fish that were larger at release were detected at a significantly higher rate the following spring and summer than their smaller cohorts $(\mathrm{P}<0.0001)$.

5) Wild fish migrating in April and May were significantly larger at release than fish migrating after May $(\mathrm{P}<0.0001)$.

6) At McNary Dam in 1995. the 62 wild chinook salmon smolts that were weighed and measured grew an average of $42.2 \mathrm{~mm}$ in length and gained an average of $10.0 \mathrm{~g}$ in weight over an average of 284.1 days.

7) In 1995. migration timing of wild spring/summer chinook salmon smolts at Lower Granite Dam was unique compared to previous years. with peak passage on 9 May. However. as observed in previous l-ears. peak detections of fish from individual streams in 1995 
occurred over an extended period. During the 6 years before 1995, passage timing of wild fish at this dam was highly variable and generally independent of river flows before mid-May. In contrast. during this period. peak passage of wild fish after about mid-May tended to coincide well with periods of peak river flow. In 1995. sustained high flows from mid-May to early June moved wild fish through the dam at a more uniform rate than in previous years. and over $90 \%$ had passed when peak flows occurred at the dam on 6 June.

8) Before 1995. we observed a 2-week shift in timing of wild fish at Lower Granite Dam between relatively warm and relatively cold years. In the colder-than-normal years of 1989. 1991. and $1993,50 \%$ of all wild fish passed the dam by mid-May, while $90 \%$ passed by mid-June (except 1993 when high flows moved 90\% through the dam by the end of May). In the warmer-than-normal years of 1990. 1992. and 1994. 50\% of all wild fish passed this dam from 29 April to 4 May. and $90 \%$ passed by the end of May. In 1995, we experienced near-normal weather conditions in late winter and early spring and observed intermediate passage timing at the dam (compared to previous years) with 50 and 90\% passage on 9 May and 5 June. respectively.

9) Die1 timing patterns of wild chinook salmon smolts exiting from the fish and debris separators varied among the dams. At Lower Granite and Lower Monumental Dams. more wild fish exited the separators during nighttime hours $(1800-0600 \mathrm{~h})$ than exited during the day (0600-1800 h). but the difference was significant only at Lower Granite Dam. At both Little Goose and McNary Dams. more wild fish exited the separators during daytime hours than exited at night. but the difference was significant only at Little Goose Dam. 


\section{INTRODUCTION}

\section{Project Goals}

The goals of this study are to 1) characterize the migration timing of different wild stocks of Snake River spring/summer chinook salmon smolts at dams on the Snake and Columbia Rivers, 2) determine if consistent patterns are apparent, and 3) determine what environmental factors influence migration timing.

\section{Background}

The National Marine Fisheries Service (NMFS) began a cooperative study with the U.S. Army Corps of Engineers (COE) in 1988 to mark wild Snake River spring and summer chinook salmon parr with Passive Integrated Transponder (PIT) tags for transportation research. This project continued through mid-1991. with migrating smolts monitored as they passed Lower Granite. Little Goose. and McNary Dams during spring and summer 1989-1991 (Matthews et al. 1990, 1992; Achord et al. 1992). Information from this study demonstrated that the timing of various wild stocks through Lower Granite Dam differed among streams of origin and also differed from patterns for hatchey-reared fish. Generally. the migrations of wild spring chinook salmon were later and more protracted than those of their hatchery-reared counterparts. and they also exhibited variable timing patterns over the 3 years. Conversely. the migrations of wild summer chinook salmon were earlier and more protracted than those of their hatchery counterparts. 
The present study began with the 1992 migration of wild chinook salmon smolts (Achord et al. 1994). Warmer-than-normal weather and higher-than-normal water temperatures in late winter and spring appeared to elicit an early migration timing for all wild smolts in 1992. The migration timing of wild spring chinook salmon smolts in 1992 was earlier than for the previous 3 years. Also. most wild summer chinook salmon smolts migrated earlier than wild spring chinook salmon smohs. However. as was observed during previous years, all wild stocks exhibited protracted and variable migration timing at Lower Granite Dam.

In 1993. cooler-than-normal weather and low water temperatures from late winter to early summer appeared to elicit a late migration timing: however. high flows during the third week of May moved a large portion of wild spring/summer chinook salmon through the dams (Achord et al. 1995a). As observed in previous years. wild stocks also exhibited variable migration timing at Lower Granite Dam; however. the middle 80\% passage time of wild fish at the dam was more compressed in 1993 than in earlier years.

In 1994. migration timing of wild spring/summer chinook salmon smolts at Lower Granite Dam was similar to timing in 1990 and 1992. with peak passage in all 3 years in April; however. peak detections of fish from individual streams in 1994 occurred from late April to late May. As obsened for 1990 and 1992. 1994 was also warmer than normal during late winter and spring.

Prior to 1992. decisions on dam operations and use of stored Water relied on recoveries of branded hatchery fish. index counts at traps and dams. and flow patterns at the dams. In 1992. a more complete approach integrated PIT-tag detections of several wild spring and summer chinook salmon stocks at Lower Granite Dam. We initiated a database on wild fish, 
which addresses several goals of the Columbia River Basin Fish and Wildlife Program of the Pacific Northwest Electric Power Planning Council and Conservation Act (1980). Section 304(d) of the program states: "The monitoring program will provide information on the migrational characteristics of the various stocks of salmon and steelhead within the Columbia Basin." Further. Section 20 l (b) urges conservation of genetic diversity. This will only be possible if wild stocks are preserved. The advent of PIT-tag technology has provided the opportunity to precisely track the smolt migrations of many stocks as they pass through the hydroelectric comples on their way to the ocean.

This report provides information on PIT tagging of wild chinook salmon parr in 1994. and the subsequent monitoring of these fish. Fish were monitored as they migrated through some juvenile migrant traps in 1994 and 1995 as well as Lower Granite. Little Goose. Lower Monumental. McNary. John Day. and Bonneville Dams during 1995.

\section{FISH COLLECTION AND TAGGING}

In 1992. Oregon Department of Fish and Wildlife (ODFW) began PIT tagging wild chinook salmon parr in the Grande Ronde and Imnaha River drainages in northeast Oregon. All tagging. detection. and timing information for fish from these streams in 1994-1995 will be reported by ODFW. However. with ODFW's concurrence. NMFS will continue to report the timing at Lower Granite Dam of fish from those streams in Oregon where we PIT tagged wild chinook salmon from 1988 to 1991.

We collected and PIT tagged wild chinook salmon parr from various reaches of each targeted stream during July. and August 1994 (Fig. 1). Our primary objective was to collect 


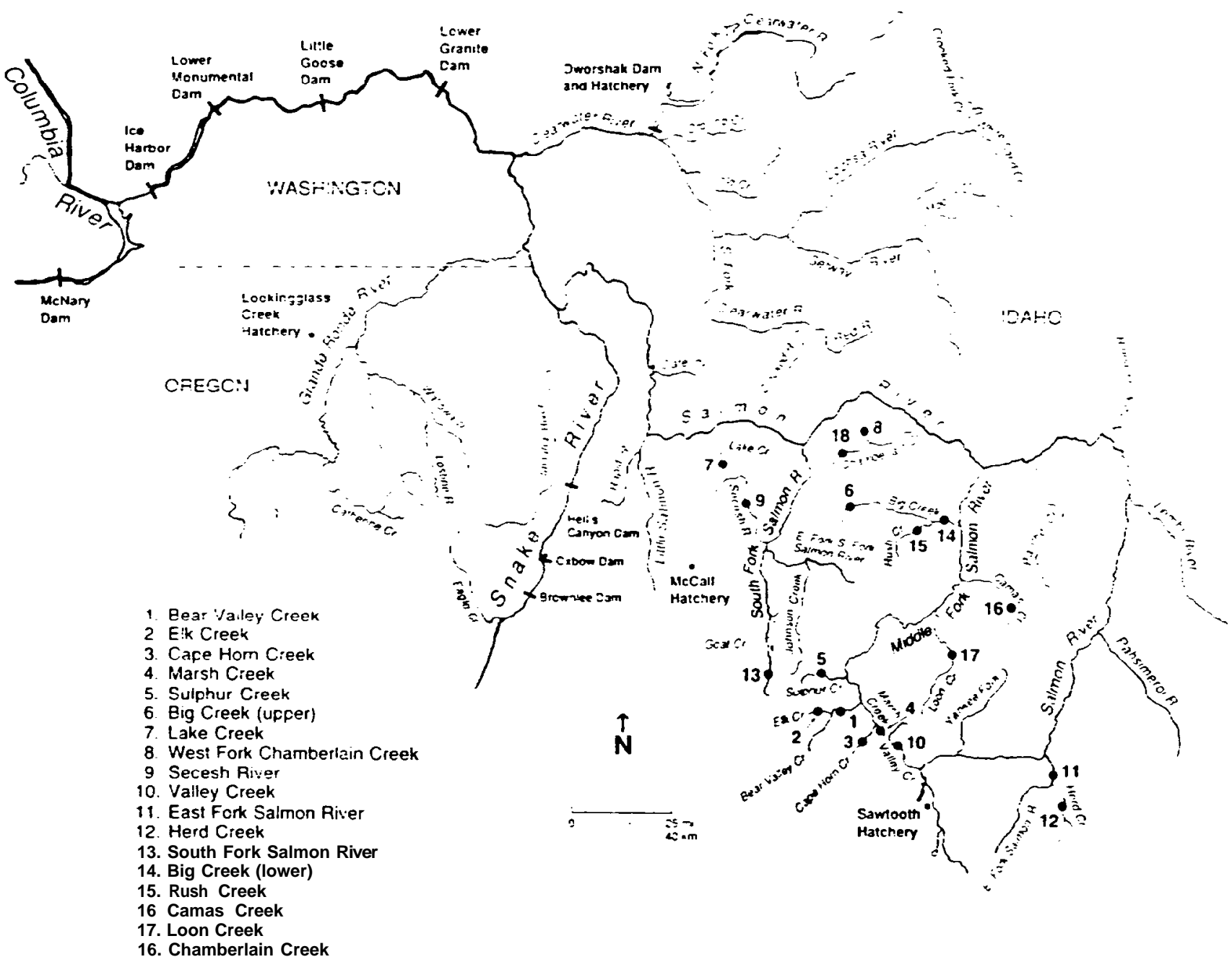

Figure i. Study area where wild spring/summer chinook salmon parr were PIT tagged during summer 1994 
pat-r in these streams quickly and with minimal impact to the fish. Areas of high parr concentrations were located by snorkeling in advance of collection. Thus. we concentrated our collection and marking efforts in areas within each stream where parr abundance was highest.

Collection and PIT-tagging procedures described by Matthews et al. (1990) and Achord et al. (1994. 1995a. 1995b) were used for our field work in 1994.

From 27 July to 24 August 1994. we collected 24.874 wild chinook salmon parr in Idaho over a distance of about 50 stream kilometers (Table 1 and Appendix Table 1). Of these. 18.459 fish were PIT tagged and released back into the streams. Numbers tagged and released per stream ranged from 15 in Rush Creek to 1.575 in Marsh Creek. Fork lengths of tagged and released wild fish ranged from 45 to $118 \mathrm{~mm}$ (mean $65 \mathrm{~mm}$ ). Weights ranged from 1.2 to $12.0 \mathrm{~g}$ (mean $3.6 \mathrm{~g}$ ).

Table 2 provides a summary of species other than chinook salmon observed during electrofishing or seining operations. The most abundant of these was sculpin. We caution that the numbers of fish in Table 2 do not represent abundance of all other fish in the areas of collection.

Mortality associated with collection and tagging procedures was $10 \mathrm{w}$. and 24-hour tag loss was zero (Table 3 and Appendix Table 2). Average collection mortality was $1.3 \%$ (all collection mortality occurred during electrofishing). average tagging mortality was $0.1 \%$. and average 24-hour delaved mortality was $0.5 \%$. The average overall observed mortality was $1.5 \%$. 
Table 1. Summary of wild chinook salmon parr collected, PIT tagged and released; average fork lengths and weights; and approximate distances covered in streams of Idaho during July and August 1994.

\begin{tabular}{|c|c|c|c|c|c|}
\hline Tagging location & $\begin{array}{l}\text { Numbcr } \\
\text { collected }\end{array}$ & $\begin{array}{l}\text { Number tagged } \\
\text { and released }\end{array}$ & $\begin{array}{l}\text { Av erage length } \\
\text { of tagged fish }(\mathrm{mm})\end{array}$ & $\begin{array}{l}\text { Average weight } \\
\text { of tagged fish (g) }\end{array}$ & $\begin{array}{c}\text { Kilometers } \\
\text { covered in } \\
\text { streams }\end{array}$ \\
\hline Brar Valley Creek & 1.558 & 1.455 & 63 & 3.5 & 5 \\
\hline Elk Creek & 1.542 & 1.512 & 67 & 4.1 & 4 \\
\hline Sulphur Creek & 769 & 728 & 62 & 3.0 & 2 \\
\hline Marsh Creek & 1.680 & 1.575 & 69 & 4.0 & 3 \\
\hline Cape horn Creek & 3.833 & 1.443 & 62 & 2.6 & 1 \\
\hline Valley Creek & 1.944 & 1.552 & 64 & 3.7 & 2 \\
\hline Camas Creek & 1.986 & 1.528 & 61 & 3.2 & 3 \\
\hline Loon Creek & 1.023 & $96-\mathrm{t}$ & 65 & 3.5 & 2 \\
\hline Herd Creek & 568 & 531 & 74 & 3.9 & 2 \\
\hline E. Fork Salmon River & 1.070 & 986 & 74 & 5.4 & 3 \\
\hline Big Creek (upper) & 862 & 757 & 62 & 3.4 & 3 \\
\hline S. Fork Salmon River & 4.017 & 1.569 & 59 & 2.6 & 5 \\
\hline Big Creek (lower) & 757 & 727 & 75 & 5.3 & 4 \\
\hline Rush Creek & 15 & 15 & 75 & - & 2 \\
\hline W. Fork Chamberlain Creek & 928 & 917 & 66 & 3.3 & 2 \\
\hline Chamberlain Creek & 266 & 241 & 65 & - & 2 \\
\hline Secesh River & 1.593 & 1.551 & 63 & 3.2 & 3 \\
\hline Lake Creek & 463 & 405 & 63 & - & 2 \\
\hline Totals or av erages & 24.874 & 18.459 & 65 & 3.6 & 50 \\
\hline
\end{tabular}


Table 2. Summay of species other than chinook salmon observed during collection operations in various Idaho streams. July and August 1994.

\begin{tabular}{|c|c|c|c|c|c|c|c|c|}
\hline Stream & Steelhead & $\begin{array}{l}\text { Brook } \\
\text { trout }\end{array}$ & Whitefish & $\begin{array}{c}\text { Curthraat } \\
\text { trout }\end{array}$ & $\begin{array}{l}\text { Bull } \\
\text { trout }\end{array}$ & Sculpin & Dace & Sucker \\
\hline Bear Valley Creek & 316 & 528 & 205 & 0 & 0 & 455 & 107 & 0 \\
\hline Elk Creek & 161 & 430 & $\Sigma$ & 0 & 0 & 209 & 2 & 0 \\
\hline Sulphur Creek & 367 & 0 & 151 & I & 0 & 1.782 & 7 & 0 \\
\hline Marsh Creek & 202 & 174 & 253 & 0 & 0 & 156 & 2 & 0 \\
\hline Cape Horn Creek & 2 & 96 & 3 & 0 & 0 & 154 & ᄀ & 0 \\
\hline Valley Creek & 17 & 220 & 67 & 0 & 0 & 338 & S-I & 0 \\
\hline Camas Creek & 1.076 & 1 & I & 0 & $n$ & 0 & 0 & 0 \\
\hline Loon Creek & 797 & 1 & I & & 8 & 1.151 & 0 & 0 \\
\hline herd Creek & 388 & 0 & 2 & 0 & ᄀ & 202 & 0 & 0 \\
\hline E. Fork Salmon River & 1.186 & 1 & & 0 & 0 & 1.187 & 0 & 0 \\
\hline Big Creel, ( upper) & 233 & 45 & $!$ & 0 & 4 & 1.862 & 0 & 0 \\
\hline S. Fork Salmon River & 388 & 21 & 282 & 3 & 0 & 369 & 3 & 0 \\
\hline Big Creek lower ) & 994 & & 0 & 3 & 0 & 2248 & 2.425 & 0 \\
\hline Rush Creek & & $\cdots \cdot$ & - & & .. & ----- & $-\cdots$ & -- \\
\hline W. Fork Chamberlain Creek & 362 & 0 & 52 & 0 & $I-I$ & 95 & 0 & 0 \\
\hline Chamberlain Creek & 219 & 0 & 50 & 0 & 0 & 350 & 0 & 0 \\
\hline Secesh riv er & 312 & Ђ & 4 & 0 & 0 & 386 & 169 & 0 \\
\hline Lake Creek & $1-1$ & 78 & $\underline{22}$ & 0 & 6 & 410 & 9 & 0 \\
\hline Totals & 7.162 & 1.747 & I.162 & 2 & 34 & 11.354 & 2.810 & 0 \\
\hline
\end{tabular}

${ }^{3}$ Big Creek (lower) numbers of fish includes numbers collected in Rush Creek. 
Table 3. Mortality and tag loss for wild chinook salmon parr collected and PIT tagged in Idaho. July and August 1994.

\begin{tabular}{|c|c|c|c|c|c|}
\hline \multirow[b]{2}{*}{ Tagging location } & \multicolumn{4}{|c|}{$\begin{array}{l}\text { Mortality } \% \\
\end{array}$} & 24-hour \\
\hline & Collection & Tagging & 24-hour & Overall tag & $\operatorname{loss}(\%)$ \\
\hline Bear Valley Creek & 1.7 & 0.2 & 0.4 & 2.0 & 0.0 \\
\hline Elk Creek & 1.7 & 0.1 & 0.0 & 1.8 & 0.0 \\
\hline Sulphur Creek & 0.4 & 0.0 & 0.0 & 0.4 & 0.0 \\
\hline Marsh Creek & 0.2 & 0.9 & 0.0 & 1.1 & 0.0 \\
\hline Cape Horn Creek & 0.1 & 0.0 & 0.6 & 0.2 & 0.0 \\
\hline Valley Creek & 0.3 & 0.9 & 9.9 & 0.3 & 0.0 \\
\hline Camas Creek & 27 & 0.0 & 0.7 & 3.0 & 0.0 \\
\hline Loon Creek & 2.1 & 0.0 & --- & 2.1 & --- \\
\hline Herd Creek & 4.8 & 0.9 & --- & $4 . S$ & --- \\
\hline E. Fork Salmon River & 7.1 & 0.0 & --- & 7.4 & --- \\
\hline Big Creek (upper) & 1.1 & 0.0 & --- & 1.4 & --- \\
\hline S. Fork Salmon River & 0.6 & 0.1 & 0.8 & 0.7 & 0.0 \\
\hline Big Creek (lower) & 3.8 & 0.0 & --- & 3.8 & --- \\
\hline Rush Creek & 0.0 & 0.0 & --- & 0.0 & --- \\
\hline W. Fork Chamberlain Creek & 0.0 & 0.0 & --- & 0.0 & --- \\
\hline Chamberlain Creek & 0.4 & 0.0 & --- & 0.4 & --- \\
\hline Secesh River & 0.6 & 0.0 & --- & 0.6 & --- \\
\hline Lake Creek & 0.2 & 0.2 & --- & 0.4 & --- \\
\hline Averages & 1.3 & 0.1 & 0.5 & 1.5 & 0.0 \\
\hline
\end{tabular}




\section{DETECTIONS AT TRAPS}

During fall 1994. juvenile migrant fish traps were operated on Marsh Creek and the South Fork of the Salmon River. During spring 1995. juvenile migrant fish traps were operated on the lower Salmon River near Whitebird. Idaho. and the Snake River at Lewiston. Idaho. All traps were operated by the Idaho Department of Fish and Game.

A total of 158 previously PIT-tagged wild spring/summer chinook salmon from Idaho were detected at the 4 juvenile migrant fish traps combined in fall 1993 and spring 1995. A total of 122. 12. 15. and 9 were detected at the Marsh Creek trap. the Salmon River trap. the South Fork Salmon River trap. and the Snake River trap. respectively. At the migrant trap on Marsh Creek. 56 of the 122 fish detected were weighed and measured. They had grown an average of $7.7 \mathrm{~mm}$ in length (range $0-23 \mathrm{~mm}$ ) and gained an average of $\mathrm{I} .3 \mathrm{~g}$ in weight. with an average of 51.2 days between measurements. The overall average length of released fish from Marsh Creek in summer $(69 \mathrm{~mm})$. was the same as the overall averagc length at release for fish detected at the Marsh Creek trap in the fall. Of the 15 fish detected at the South Fork of the Salmon River trap. 3 were measured and had grown an average of $7.0 \mathrm{~mm}$ (range 1-1 3 $\mathrm{mm}$ ) over an average of 55.6 days between measurements. So measurements were recorded on the remaining 21 PIT-tagged fish detected at the 2 downstream juvenile migrant fish traps on the Salmon and Snake Rivers in spring 1995.

\section{DETECTIONS AT DAMS}

During spring summer. and fall 1995. surviving chinook salmon PIT tagged for this study migrated volitionally downstream throug,= the hydroelectric complex on the Snake and 
Columbia Rivers. Of the eight dams the smolts passed. four were equipped with complete smolt collection and PIT-tag monitoring systems: Lower Granite. Little Goose. and Lower Monumental Dams on the Snake River. and McNary Dam on the Columbia River (Fig. I). Two additional dams below McNary Dam. John Day and Bonneville Dams. were equipped with PIT-tag detection gear within their sub-sampling systems.

At the four smolt collection dams. all smolts guided away from the turbine intakes and into the juvenile bypass systems were electronically interrogated for PIT tags as they passed through the distribution flumes downstream from the outlet orifices of the fish and debris separators. The PIT-tag monitor systems were the same as those described by Prentice et al. (1990). Dates and times to the nearest second were recorded on a computer as PIT-tagged fish passed through the numbered detector coils in the fish distribution flumes. All detection data were transferred once each day to the mainframe computer operated by the Pacific States Marine Fisheries Commission in Portland. Oregon.

Since the PIT-tag detection/diversion systems (Matthews et al. 1990. 1992; Achord et al. 1992) were operational at Lower Granite. Little Goose. Lower Monumental. and McNary Dams throughout the 'migration season. most PIT-tagged fish were diverted back to the river below these dams. Therefore. to accurately portray timing at the dams for the various wild stocks of fish. we used first-time detections at each dam and adjusted these detections daily for spill. The equation used to adjust the daily detections for individual streams and 
combined populations at each dam was

number detected

$\mathrm{X}$

$=$

average daily powerhouse flow

average daily flow spilled

with Xrounded to the nearest whole number and added to the number detected to produce an adjusted number of PIT-tagged fish passing each dam daily for individual or combined populations!.

From 10 April to 22 September 1995. an adjusted total of 2.173 fish PIT tagged in Idaho were detected (first-time) at the 6 dams (Table 4 and Appendix Tables 4.A-2 1 B). Based upon the number of parr PIT tagged and released in 199-1 (18.459). the overall average adjusted percentage of first-time detections at the six dams was $11.8 \%$. with averages of 6.5. 2.9. 1.6. 0.7. 0.0. and $0.0 \%$ at Lower Granite. Little Goose. Lower Monumental. McNary. John Day. and Bonneville Dams. respectively. The proportions of adjusted total fish detected at the sis dams were 55.4. 24.9. 14.0 5.7. 0.0. and 0.0\% for Lower Granite. Little Goose. Lower Monumental. McNary John Day. and Bonneville Dams. respectively. The overall detection rates at the four collector dams varied by stream of origin (Fig. 2 and Table 4). ranging from $6.3 \%$ of the Valley Creek fish to $28.9 \%$ of the Big Creek (lower) fish.

Due to rounding. total adjusted numbers for daily detections of fish from combined streams in Appendix Tables 23-26 will not add up to the total adjusted detections for individual streams in Table 4. 
Table 4. Summary of' first-time detections and detections adjusted for spill of PIT-tagged wild spring/summer chinook salmon smolts from Idaho at four dams from April to October 1995 See Table $t$ for numbers released.

\begin{tabular}{|c|c|c|c|c|c|c|c|c|c|c|c|c|}
\hline \multirow[b]{4}{*}{ SIream } & \multicolumn{12}{|c|}{ xetectioms } \\
\hline & \multicolumn{3}{|c|}{ L.uwer (iranitc Dam } & \multicolumn{3}{|c|}{ I ittle goose dam } & \multicolumn{3}{|c|}{ I ower monumental dam } & \multicolumn{3}{|c|}{ McNary Dam } \\
\hline & \multirow[t]{2}{*}{ Unadjusted } & \multicolumn{2}{|c|}{$\lambda$ Adjusted } & \multirow[t]{2}{*}{ Inadjusicd } & \multicolumn{2}{|c|}{ Adiusted } & \multirow[t]{2}{*}{ Inadjusked } & \multicolumn{2}{|c|}{ Adjusted } & \multirow[t]{2}{*}{ Inadjusled } & \multicolumn{2}{|c|}{ Adjusted } \\
\hline & & $\mathbf{N}$ & "0 & & $\mathbf{N}$ & $0_{0}$ & & $\mathbf{N}$ & $\%$ & & $\mathbf{N}$ & $\%$ \\
\hline Bear Valley Creek. & 73 & 82 & 56 & 18 & 20 & 14 & 14 & 14 & 10 & 2 & & 02 \\
\hline Ek Creek & 75 & 80 & 53 & 28 & 31 & 20 & 16 & 17 & 11 & 3 & 6 & 04 \\
\hline Sulphur Creek & 56 & 61 & 8.4 & 34 & 41 & 50 & IX & $\boldsymbol{2 1}$ & 29 & 3 & 5 & 07 \\
\hline Marsh Creek & 103 & । 16 & 7.4 & 35 & 46 & 29 & 21 & 23 & 15 & $"$ & 17 & 11 \\
\hline Cape I hom Creek & 84 & 95 & 66 & 39 & 47 & 3.3 & 19) & $\mathbf{2 1}$ & 15 & " & 12 & 08 \\
\hline Valey Creek & 50 & 52 & 33 & 24 & 24 & 15 & 19 & 19 & 12 & $\therefore$ & 3 & 02 \\
\hline camas creek & 59 & 66 & 43 & 32 & 40 & 26 & 25 & 27 & 18 & 6 & 12 & 08 \\
\hline Loon Creek & 83 & 95 & 48 & 40 & 52 & 54 & 30 & 33 & 34 & 2 & 4 & 04 \\
\hline Herd Creck & 36 & 38 & 71 & 17 & 27 & 39 & 10 & 11 & 21 & 2 & 3 & 06 \\
\hline 1. fork Salmon River & 69 & 71 & 72 & 16 & 20 & 20 & 6 & 6 & 06 & 4 & 7 & 07 \\
\hline Big Creek (upper) & 50 & 56 & 74 & 23 & 25 & 3.3 & 10 & 17 & 22 & 4 & 8 & 11 \\
\hline S Fork Salmon River & 78 & 88 & 56 & $\mathbf{2}$ & 27 & 17 & (1) & 20 & 11 & 6 & II & 07 \\
\hline Big Creek (lower) & $1 \mathbf{1 2}$ & 121 & 16. 6 & 37 & 50 & 69 & 22 & 26 & 36 & & 13 & 18 \\
\hline Rush C'reek & 2 & 2 & 133 & & 1 & 67 & । & 1 & 67 & 0 & 0 & 00 \\
\hline W Fork Chamberlain C recth & 43 & 48 & 52 & ? & 24 & 26 & 7 & 8 & 09 & 2 & & 03 \\
\hline Chamberlain Creek & 14 & 15 & 62 & II & 14 & 5.8 & 5 & 5 & 21 & 0 & 0 & 00 \\
\hline Secesh River & 86 & 92 & 54 & 44 & 52 & $3 i$ & 28 & 31 & 20 & 8 & 14 & 09 \\
\hline Lake Creek & 24 & 26 & 6.4 & -0 & -6 & 1.5 & -4 & 4 & 10 & 1. & $\underline{2}$ & 0.5 \\
\hline Totals or averages & |.097 & 1.204 & 65 & 449 & 541 & 29 & 280 & 304 & 16 & 67 & 123 & 0.7 \\
\hline
\end{tabular}

" One additional fish from this stream had a first-time detection at J ohn Day Dam 


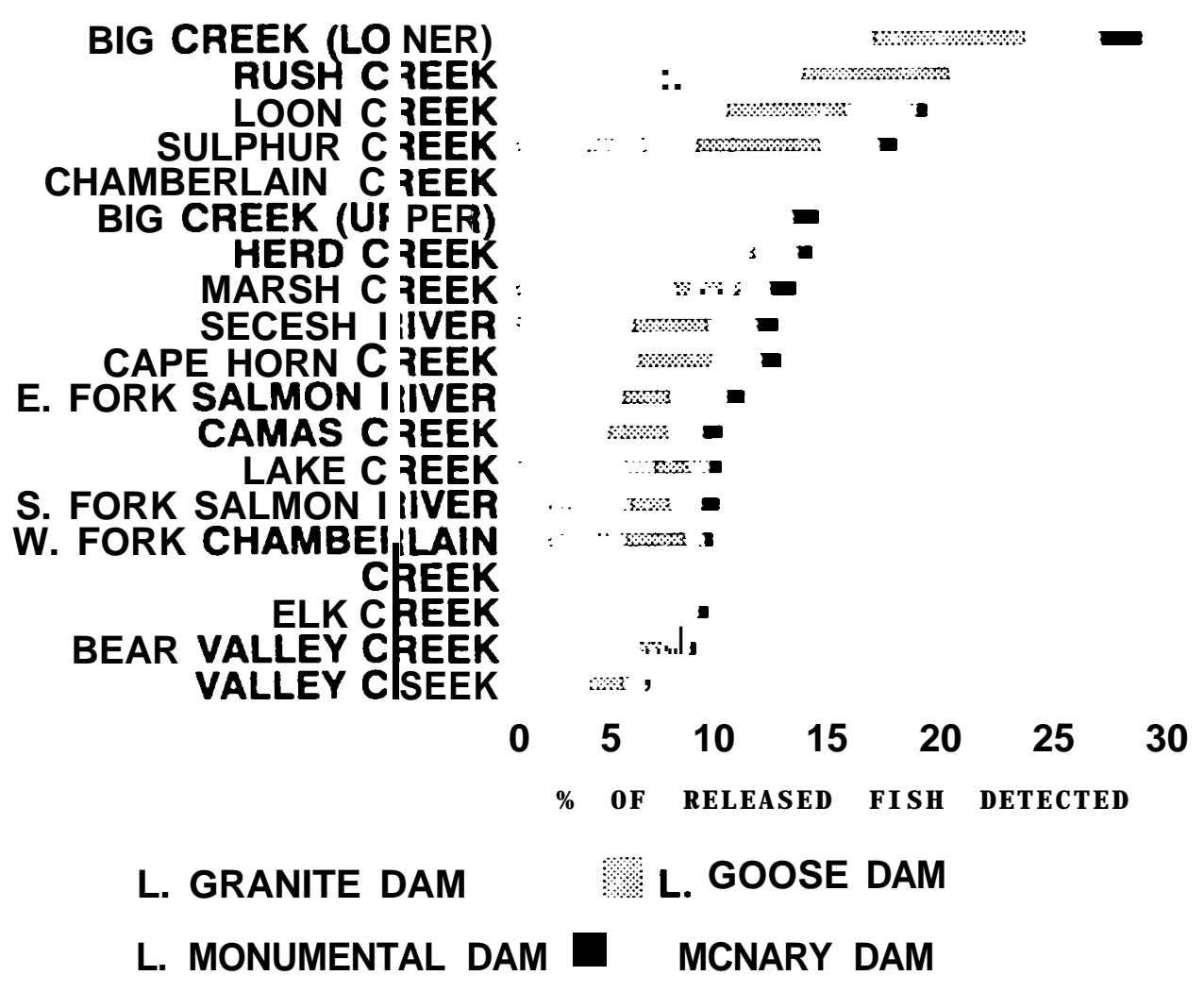

Figure 2. Percent (adjusted for spill) of PIT-tagged wild spring/summer chinook salmon smolts detected at Lower Granite. Little Goose. Lower Monumental. and McNary Dams in 1995. 
From 1990 to 1993 . the vast majority of chinook salmon parr collected in Idaho were collected by electrofishing. Therefore. we could not statistically compare subsequent detection rates at dams for released fish following electrotishing or seining. In 1994. in the South Fork of the Salmon River and Valley Creek. enough fish were collected by both collection methods to conduct a statistical analysis which compared the two methods with respect to detections at dams in 1995. We found no significant difference in detections (unadjusted) at the dams for fish released the previous year following collection by electrofishing (7.2\%) or seining (6.9\%) $(\mathrm{P}>0.05)$.

To ascertain how water temperature may have affected study fish during tagging. we examined the differences among groups in combined detection rates at dams the following spring (Appendix Table 22). The detection rate (unadjusted) of groups from all streams when tagging began with water temperatures $13^{\circ} \mathrm{C}$ or geater was $10.5 \%$. When tagging began at temperatures less than $13^{\circ} \mathrm{C}$. the detection rate was $10.1 \%$. A two-sample Z-test showed no significant difference between these percentages $(\mathrm{P}>0.05)$.

We also analyzed the detection rates on fish from groups released at different water temperatures. The detection rate of groups released when water temperatures were $13^{\circ} \mathrm{C}$ or greater was $10.0 \%$. When water temperatures were less than $13^{\circ} \mathrm{C}$. the detection rate was not significantly different $(10.6 \%)(\mathrm{P}>0.05)$.

When we added tagging and release water temperatures. we found the detection rate for groups tagged and released with additive water temperatures of $25^{\circ} \mathrm{C}$ or greater was $10.3 \%$. With additive water temperatures less than $25^{\circ} \mathrm{C}$. the detection rate was $10.2 \%$. This difference was not statistically significant $(\mathrm{P}>0.05)$. 
At release. the average fork length for all fish was $65 \mathrm{~mm}$. However. for fish detected the following spring at the dams. the average fork length at release was $67 \mathrm{~mm}$. A chi-square comparison of the length distributions showed these lengths were significantly different $(\mathrm{P}<0.0001)$. Figure 3 shows the relationship between length at release and eventual detection at the dams. Fish $63 \mathrm{~mm}$ or smaller were detected at a significantly lower rate than expected $(\mathrm{P}<0.0001)$. whereas fish $65-84 \mathrm{~mm}$ were detected at a significantly higher rate than expected $(\mathrm{P}<0.03)$.

We also found a significant difference in fork lengths at time of release between fish that migrated through the dams in April and May and fish that migrated after May $(\mathrm{P}<0.0001)$ : fish migrating after May were on average $6 \mathrm{~mm}$ smaller when released than fish migrating before this time. These data suggest that fish size may be an important factor influencing migration timing or overwintering location with respect to proximity to the first dam.

During a portion of the migration season at McNary Dam. we tested the new PIT-tag detection diversion system. The diverted fish were scanned for PIT tags. weighed. and measured. This allowed us to collect information on fork length and weight gains for wild fish from time of tagging and release until recovery at the dam (Table 5). The average gains in fork length and weight were $42.2 \mathrm{~mm}$ and $10.0 \mathrm{~g}$. respectively. over an average of 284. 1 day.

Of a total of 20 (including fish detected at previous dams) wild PIT-tagged chinook salmon smolts detected at John Day and Bonneville Dam's sub-sampling systems. 8 were measured and grew an average of $45 \mathrm{~mm}$ (range 31-67 mm). over an average of 288.8 days between measurements. 


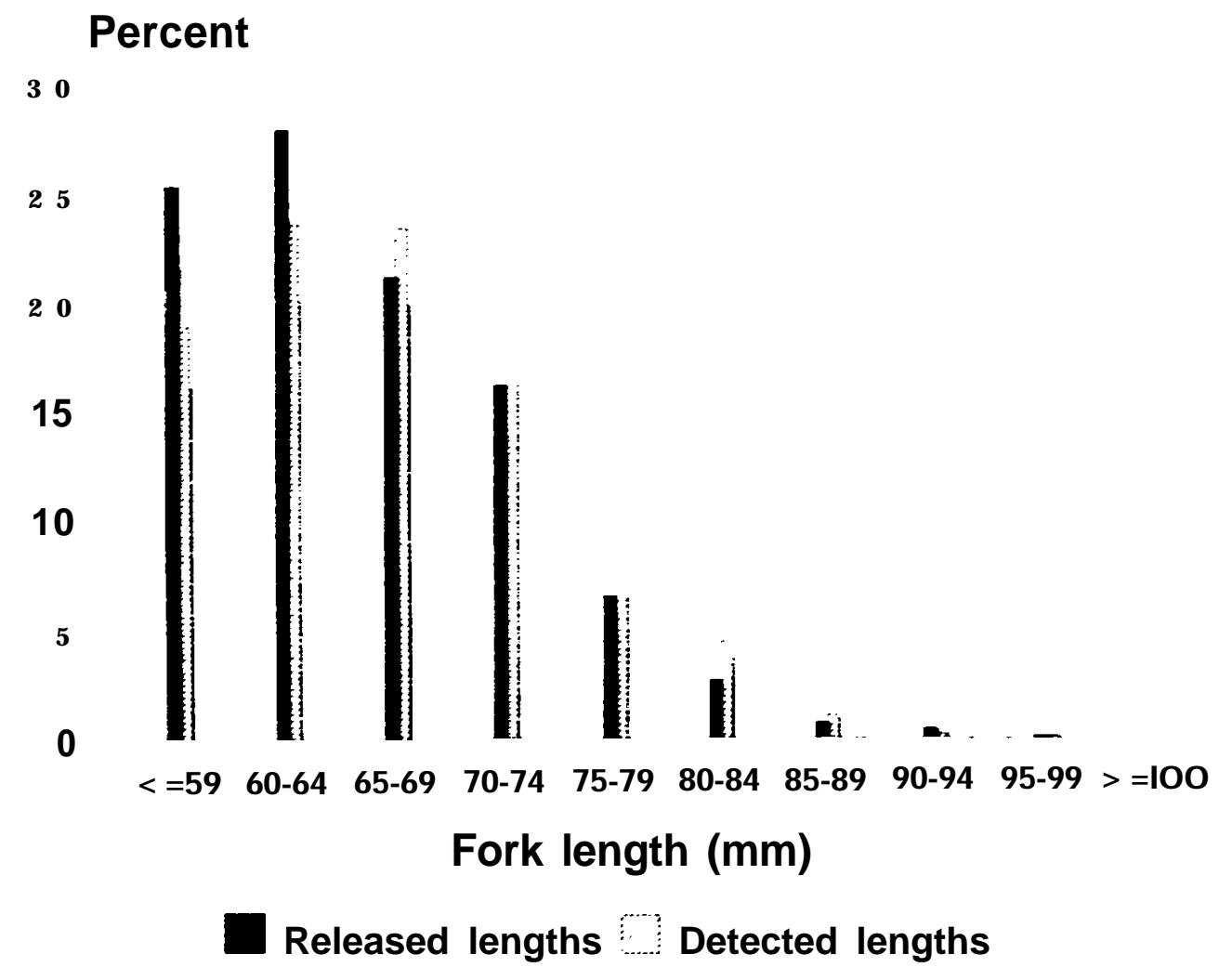

Figure 3. Percent, by fork length increments, of PIT-tagged wild spring/summer chinook salmon parr released in Idaho streams in 1994 and percent of fish detected for these length increments at Lower Granite, Little Goose, Lower Monumental, McNary, and John Day Dams in spring and summer 1995. 
Table 5. Increases in length $(\mathrm{mm})$ and weight $(\mathrm{g})$ for wild spring/summer chinook salmon from tagging in summer 1994 to recovery at McNary Dam in spring 1995 Tagged fish were recovered during PIT-tag detection/diversion tests.

\begin{tabular}{|c|c|c|c|c|c|c|c|}
\hline \multirow[b]{2}{*}{ Stream } & \multicolumn{3}{|c|}{ lenght increase } & \multicolumn{3}{|c|}{ Weight increase } & \multirow{2}{*}{$\begin{array}{l}\text { Average } \\
\text { days }\end{array}$} \\
\hline & $\mathrm{N}$ & Average & Range & $\mathrm{N}$ & Average & Range & \\
\hline Rear Valley Creek & 2 & 44.0 & $43.0-45.0$ & 1 & 12.2 & (......... & 301.2 \\
\hline Elk Creek & 7 & 42.6 & $35.0-54.0$ & 3 & to. 1 & $7.6-11.9$ & 292.2 \\
\hline Sulphur Creek & 6 & 39.5 & $34.0-49.0$ & 2 & 6.5 & $6.5-6.5$ & 296.3 \\
\hline Cape Horn Creek & 3 & 46.7 & $42.0-52.0$ & 1 & 11.6 & --------- & 299.2 \\
\hline Marsh Creek & 8 & 41.0 & $20.0-54.0$ & 3 & 11.3 & $9.9-12.6$ & 289.8 \\
\hline Valley Creek & 1 & 41.0 & -.......- & -- & $\cdots$ & $\cdots \cdots$ & 300.0 \\
\hline Camas Creek & 6 & 4522 & $23.0-56.0$ & 2 & 12.0 & to. $3-13.6$ & 281.1 \\
\hline Loon Creek & 10 & 45.5 & $31.0-60.0$ & 4 & 8.2 & $6.3-9.8$ & 287.0 \\
\hline E. Fork Salmon River & 5 & 40.0 & $32.0-49.0$ & 4 & 10.3 & $7.6-15.1$ & 271.0 \\
\hline Big Creek (upper) & 2 & 40.0 & $42.0-\mathrm{SO} .2$ & 1 & I I.7 & & 288.2 \\
\hline Big Creek (tower) & 0 & 38.2 & $31.0-41.0$ & & ---- & & 263.8 \\
\hline Chamberlain Creek & 4 & $38 . \mathrm{X}$ & $33.0-44.0$ & & & -...-..-. & 265.9 \\
\hline W. Fork Chamberlain Creek & 1 & 47.0 & -......- & & & & 275.7 \\
\hline Rush Crcek & I & 32.0 & -......... & -- & & & 262.7 \\
\hline Totals or averages & 62 & 42.2 & $23.0-60.0$ & 21 & 10.0 & $6.3-15.1$ & 284.1 \\
\hline
\end{tabular}




\section{MIGRATION TIMING AT DAMS}

Migration timing at dams was calculated by totaling the adjusted number of detections in 3-day intervals and dividing by the total adjusted detections during the season. This method was applied to detection data for fish from individual and combined streams. Timing of smolt migrations from individual streams was calculated at Lower Granite Dam (Fig. 4). while migration timing for smolts from all Idaho streams combined was calculated at all four collector dams (Fig. 5).

Fish from Big (lower)/Rush Creeks in the Middle Fork of the Salmon River drainage, Secesh River in the South Fork of the Salmon River drainage. East Fork Salmon River and Herd Creek in the upper Salmon River. and the Lostine River and Imnaha River (upper) in Oregon had the earliest timings at Lower Granite Dam (Fig. 4 and Table 6). Over 50\% of the fish from these streams passed the dam by 3 May. and most peak passage dates for fish from these streams occurred in April (Appendix Tables 12A, 13A. 16A. 17A. 20A. and Fig. 4). Fish from East Fork of the Salmon River had the earliest passage period of all streams. while fish from Big (lower)/Rush Creeks had an early and the most compressed passage period at the dam.

Fish from Bear Valley. Elk. Marsh. Cape Horn. Loon. and Camas Creeks in the Middle Fork of the Salmon River drainage. from the West Fork Chamberlain/Chamberlain Creeks tributaries of the main Salmon River. from Lake Creek and the South Fork of the Salmon River in the South Fork drainage. and from Catherine Creek in Oregon showed a later passage period at Lower Granite Dam than the aforementioned streams (Fig. 4 and Table 6). The $50 \%$ passage dates for tish from these streams ranged from 9 to 19 May. Peak passage dates 
BEAR VALLEY CREEK

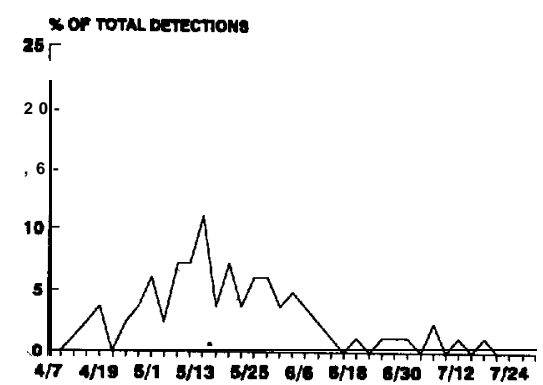

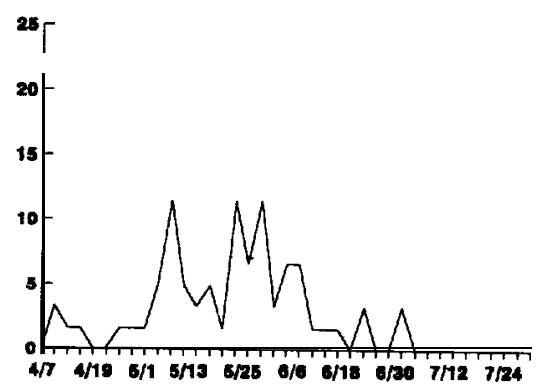

CAPE HORN CREEK

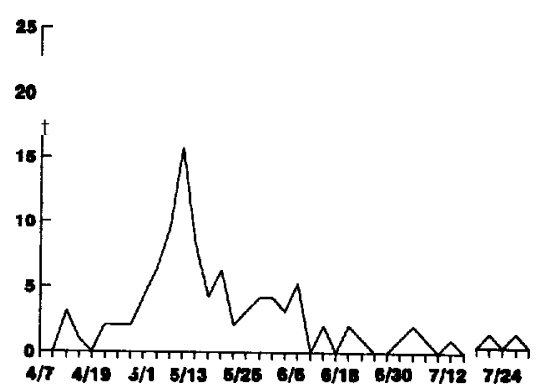

\section{ELK CREEK}

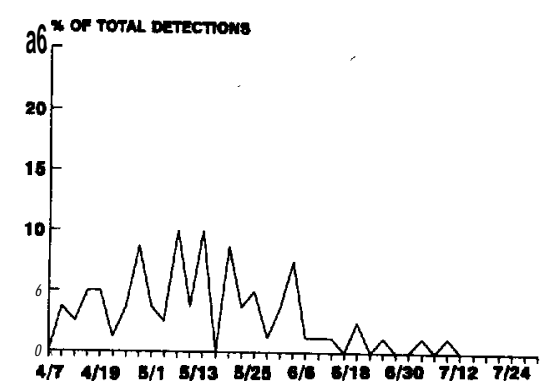

MARSH CREEK

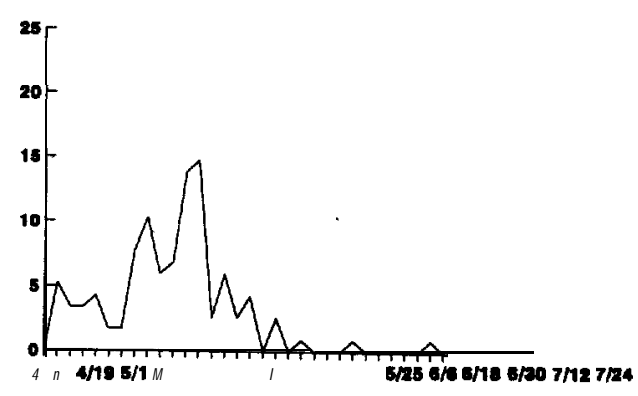

VALLEY CREEK

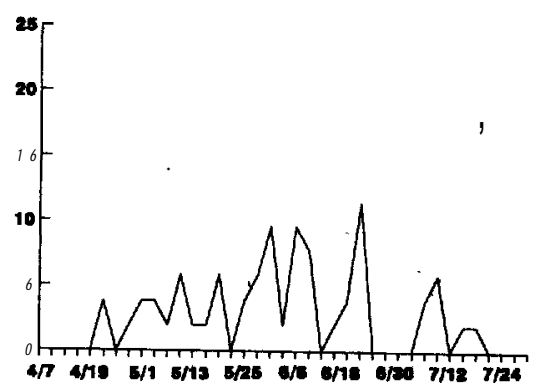

Figure 4. The migration timing (adjusted for spill) at Lower Granite Dam in 1995 of PITtagged wild spring/summer chinook salmon smolts from individual or combined streams in Idaho and Oregon. 
CAMAS CREEK

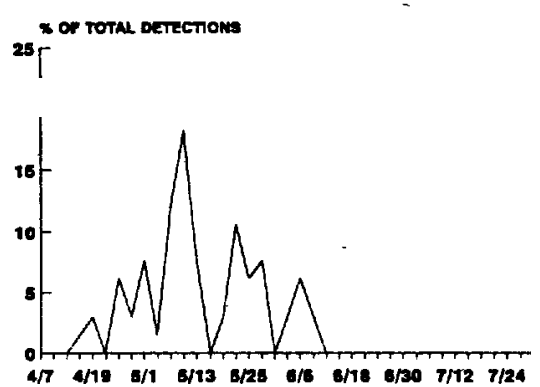

HERD CREEK

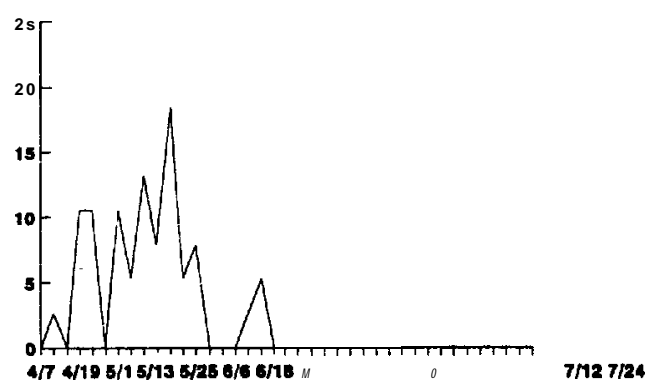

BIG CREEK (UPPER)

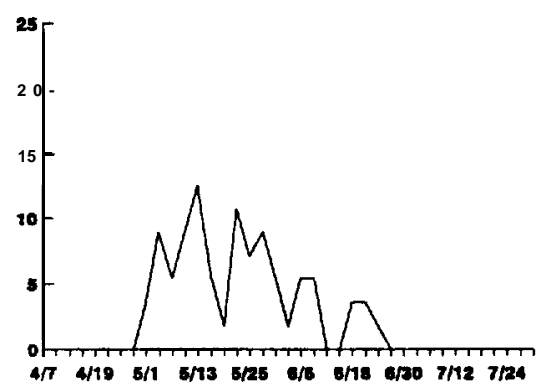

BIG CREEK (LOWER)/ RUSH CREEK

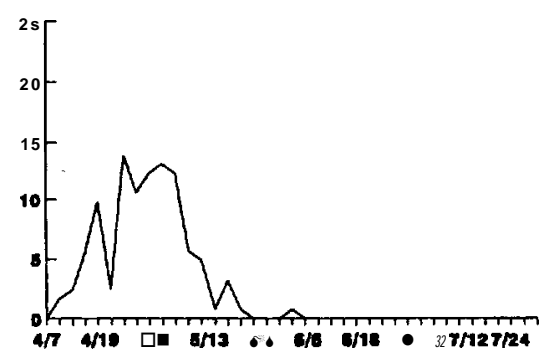

LOON CREEK

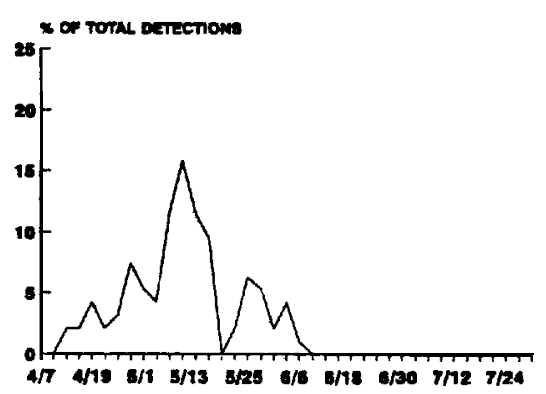

\section{EAST FORK SALMON RIVER}

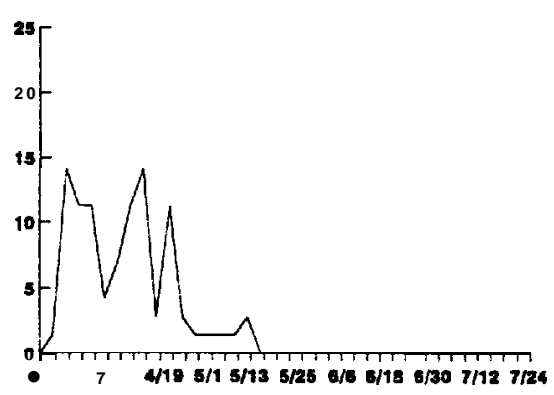

\section{SOUTH FORK SALMON RIVER}

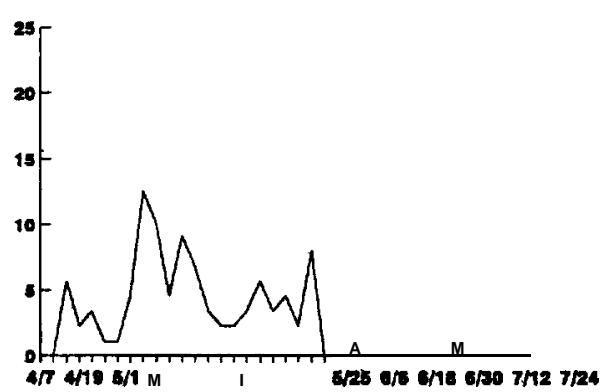

\section{WEST FORK CHAMBERLAIN /CHAMBERLAIN CREEKS}

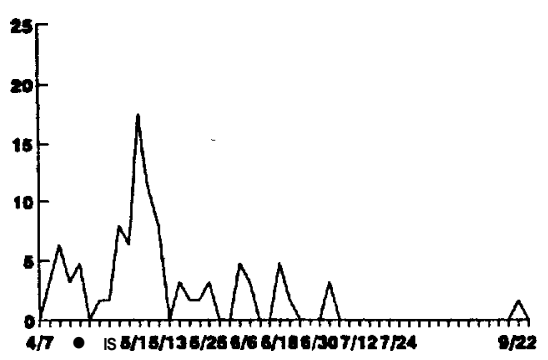

Figure 4. Continued. 
SECESH RIVER

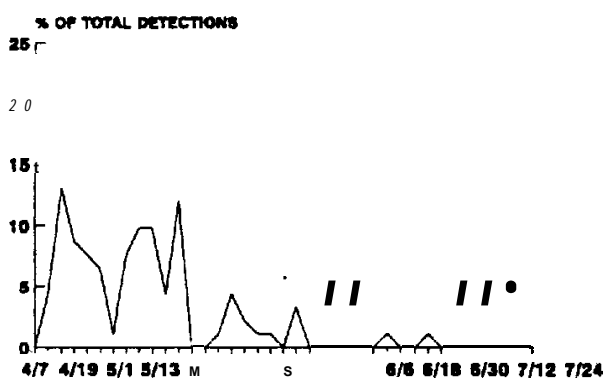

GRANDE RONDE RIVER (UPPER)

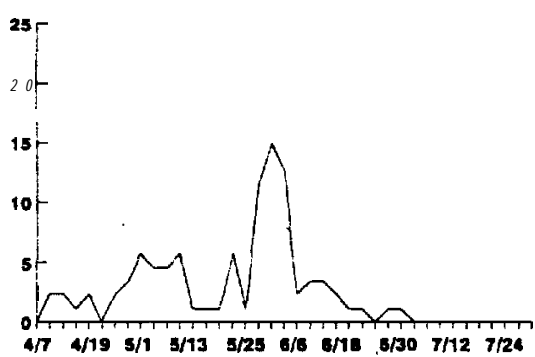

LOSTINE RIVER

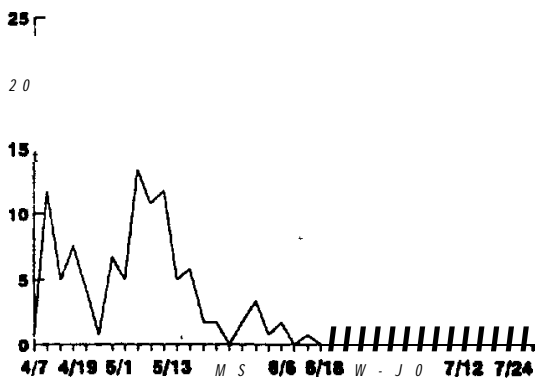

LAKE CREEK

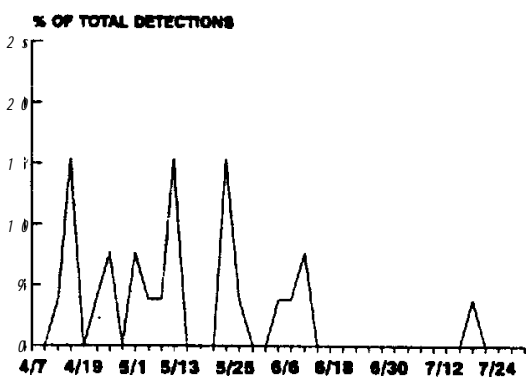

CATHERINE CREEK

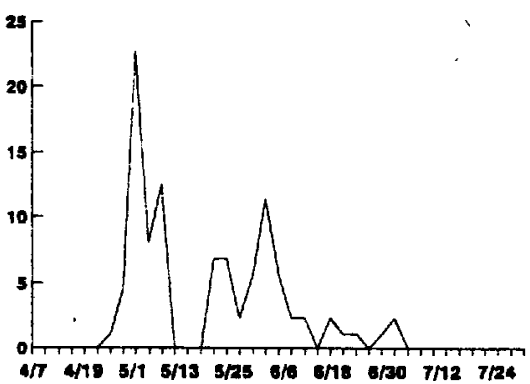

IMNAHA RIVER (UPPER)

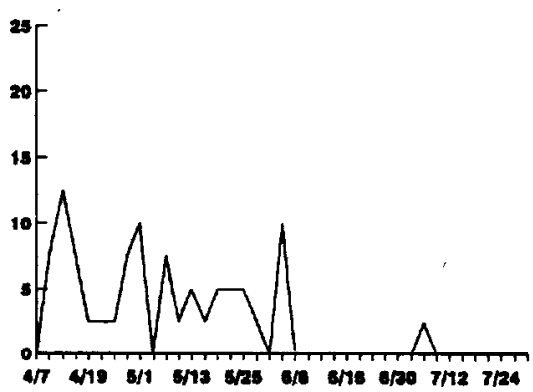

Figure 4. Continued. 


\section{Lower Granite Dam}

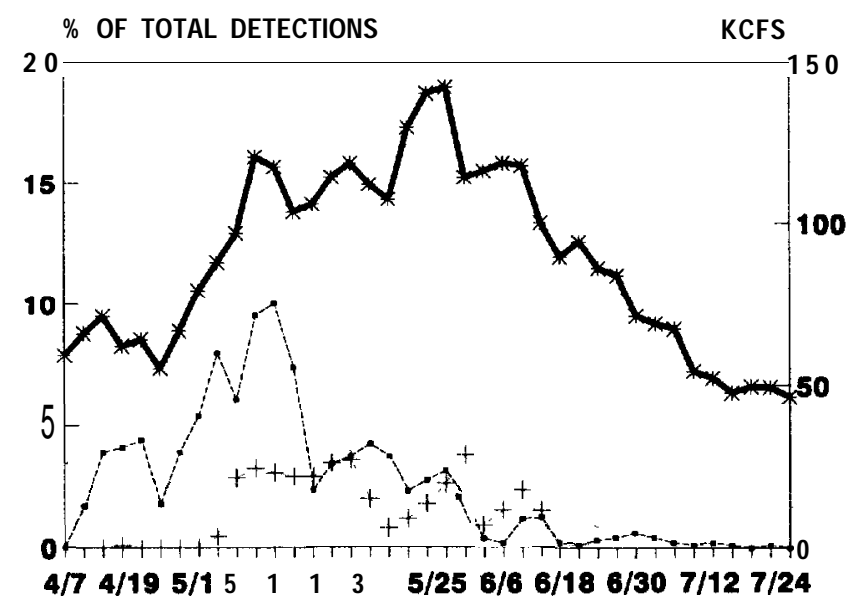

\section{Little Goose Dam}

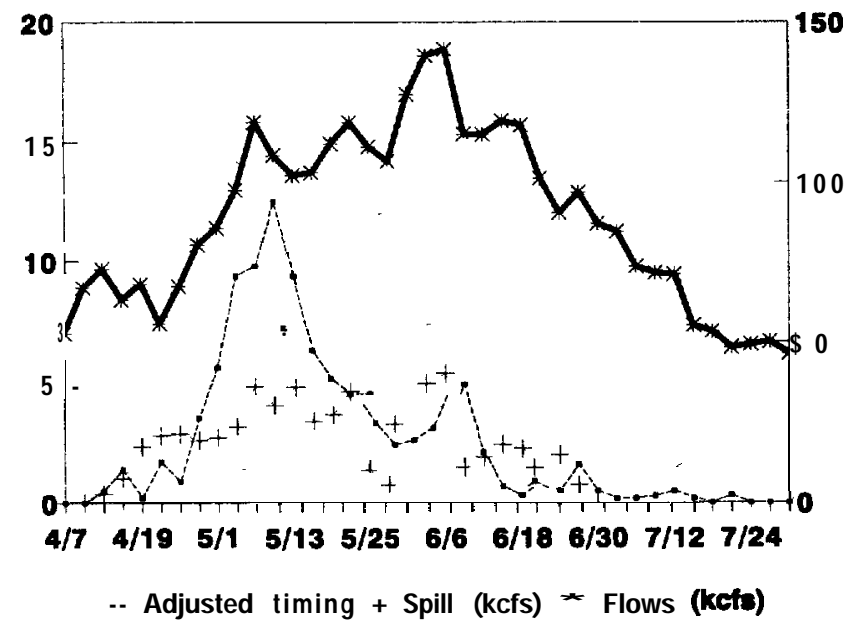

Figure 5. The overall migration timing of PIT-tagged wild spring/summer chinook salmon smolts at Lower Granite, Little Goose, Lower Monumental, and McNary Dams in 1995, with associated river spill and flows at these dams. Data represent detections from all Idaho streams combined by 3-day intervals and average river spill and flows at the dams over the same time periods. 


\section{Lower Monumental Dam}

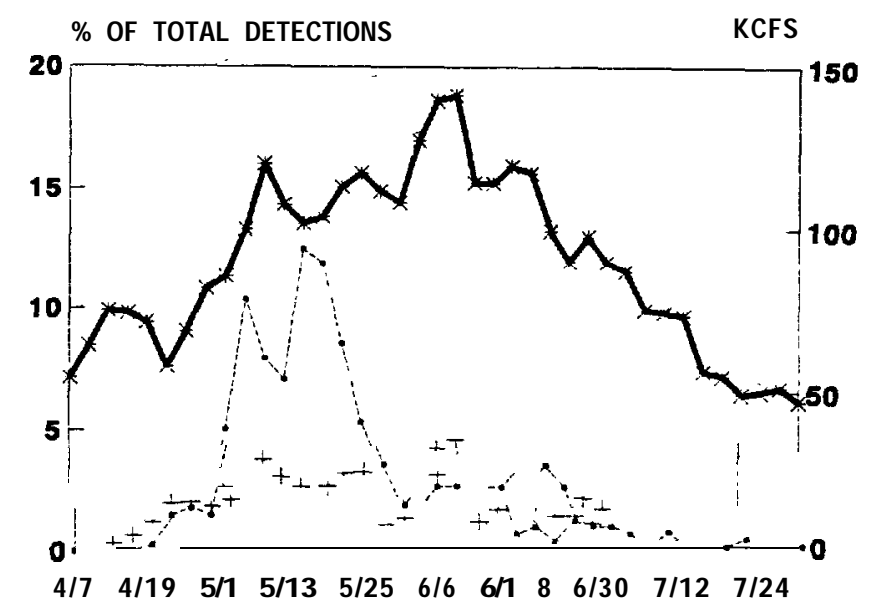

\section{McNary Dam}

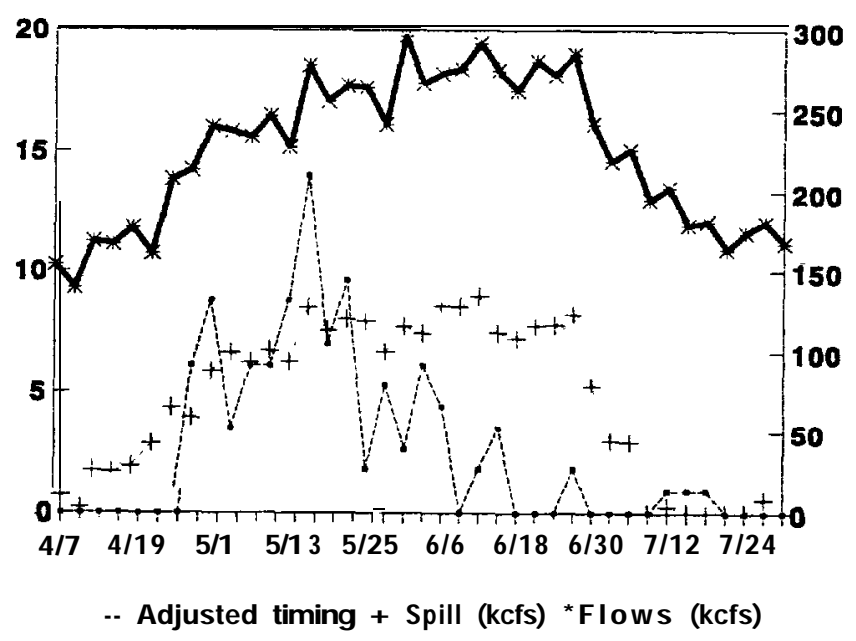

Figure 5. Continued. 
Table 6. Historical and 1995 passage dates at Lower Granite Dam for PIT-tagged wild spring/summer chinook salmon smolts from streams in Idaho and Oregon.

\begin{tabular}{|c|c|c|c|c|}
\hline \multirow[b]{2}{*}{ Year } & \multicolumn{4}{|c|}{ Passage dates at Lower Granite Dam } \\
\hline & $10 \%$ & $50 \%$ & $90 \%$ & Range \\
\hline \multicolumn{5}{|c|}{ Bear Valley Creek } \\
\hline 1990 & 19 April & 05 May & 31 May & 11 April - 18 July \\
\hline 1991 & 03 May & 20 May & 12 June & 18 April - 23 June \\
\hline 1992 & 15 April & 02 May & 24 May & 07 April - 28 June \\
\hline 1993 & 29 April & 16 May & 22 June & 22 April - 27 July \\
\hline 1994 & 22 April & 06 May & 29 May & 16 April - 15 July \\
\hline 1995 & 28 April & 18 May & 12 June & 13 April - 20 July \\
\hline \multicolumn{5}{|c|}{ Elk Creek } \\
\hline $1990 "$ & - & ------- & -...-..... & 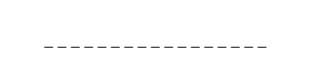 \\
\hline 1991 & 03 May & 20 May & 16 June & 25 April - 24 June \\
\hline 1992 & 11 April & 30 April & 28 May & 05 April - 17 July \\
\hline 1993 & 02 May & 16 May & 11 June & 21 April - 26 June \\
\hline 1994 & 23 April & 04 May & 21 May & 18 April - 09 July \\
\hline 1995 & 18 April & 11 May & 05 June & 10 April - 09 July \\
\hline \multicolumn{5}{|c|}{ Sulphur Creek } \\
\hline 1990 & 18 April & 30 April & 31 May & 11 April - 27 June \\
\hline $1991^{b}$ & --------- & -------- & ------- & $\longrightarrow$ \\
\hline 1992 & 16 April & 03 May & 23 May & 10 April - 01 June \\
\hline 1993 & 28 April & 16 May & 12 June & 24 April- 28 June \\
\hline $1994^{b}$ & ---- & $\mathrm{m}^{-------}$ & ----- & ---------------- \\
\hline 1995 & 02 May & 23 May & 09 June & 11 April - 09 July \\
\hline
\end{tabular}


Table 6. Continued.

\begin{tabular}{lcccc}
\hline & \multicolumn{4}{c}{ Passage dates at Lower Granite Dam } \\
\cline { 2 - 4 } Year & $10 \%$ & $50 \%$ & $90 \%$ & Range \\
\hline
\end{tabular}

Cape Horn Creek

$1990^{\circ}$

1991

1992

1993

$1994^{b}$

1995

1990

1991

1992

1993

1994

1995

1990

1991

1992

1993

1994

1995

1993

1994

1995
24 April

12 April

08 May

29 April
16 May
28 April
19 May

14 May

\section{Marsh Creek}

29 April

20 May

07 May

15 May

04 May

09 May
31 May

09 June

02 June

27 May

18 May

24 May

\section{Valley Creek}

24 April
16 April
11 May
15 April
30 April
24 April
04 May

14 May

12 June

08 May

05 June

20 May

20 June

30 April

27 May

16 May

02 June

04 May

03 June

02 June

08 July

\section{Camas Creek}

$\begin{array}{ll}1993 & 03 \text { May } \\ 1994 & 30 \text { April } \\ \mathbf{1 9 9 5} & 27 \text { April }\end{array}$

16 May

27 May

15 May

26 May

12 May

05 June

\section{May}

30 May

26 June

19 June
19 April - 06 June

10 April - 01 June

05 May - 01 July

14 April - 28 July

09 April - 17 June

09 April - 01 July

17 April - 18 June

10 April - 13 July

24 April - 10 August

16 April - 08 August

11 April - 0S July

24 April - 24 June

12 April - 29 June

21 April- 13 July

13 April - 04 June

24 April - 06 June

22 April - 09 June

22 April - 18 July

24 April - 11 July

17 April - 11 June 
Table 6. Continued.

\begin{tabular}{lllll}
\hline & \multicolumn{5}{c}{ Passage dates at Lower Granite Dam } \\
\cline { 2 - 5 } Year & $10 \%$ & $\mathbf{5 0 \%}$ & $\mathbf{9 0 \%}$ & Range \\
\hline
\end{tabular}

\section{Loon Creek}

$\begin{array}{lllll}1993 & \mathbf{0 5} \text { May } & \text { 12 May } & \text { 17 May } & \text { 03 May - 25 June } \\ 1994 & 29 \text { April } & \text { 10 May } & \text { 24 May } & \text { 22 April - 07 June } \\ \mathbf{1 9 9 5} & \mathbf{2 3} \text { April } & \mathbf{1 1} \text { May } & \mathbf{2 8} \text { May } & \text { 13 April - 0 7 June }\end{array}$

\section{East Fork Salmon River}

\begin{tabular}{|c|c|c|c|c|}
\hline 1989 & 22 April & 03 May & 18 May & 07 April - 08 June \\
\hline $1990^{b}$ & -------- & --.-- & ------- & - - - - - - - \\
\hline 1991 & 22 April & 09 May & 26 May & 16 April - 20 June \\
\hline 1992 & 13 April & 21 April & 16 May & 10 April - 03 June \\
\hline 1993 & 25 April & 06 May & 18 May & 22 April - 01 June \\
\hline 1994 & 22 April' & 28 April & 17 May & 20 April - 25 May \\
\hline 1995 & 14 April & 28 April & 10 May & 11 April - 27 May \\
\hline
\end{tabular}

\section{Herd Creek}

1992

1993

1994"

1995

14 April

26 April

18 April

$1990^{\mathrm{b}}$

1991

1992

1993

1994

1995
25 April

20 April

14 April

29 April

27 April

20 April
20 April

30 April

03 May

\section{South Fork Salmon River}

13 May

14 June

-----a--

16 May

29 April

16 May

15 May

10 May

-----m

10 June

27 May

02 June

28 June

10 June
10 May

18 May

14 May

11 April - 28 May

13 April - 18 May 26 April - 31 May
16 April - 20 June

17 April - 13 July

07 April - 27 July

26 April - 28 June

22 April - 09 July

13 April - 13 July 
Table 6. Continued.

\begin{tabular}{|c|c|c|c|c|}
\hline \multirow[b]{2}{*}{ Year } & \multicolumn{4}{|c|}{ Passage dates at Lower Granite Dam } \\
\hline & $10 \%$ & $50 \%$ & $90 \%$ & Range \\
\hline \multicolumn{5}{|c|}{ Big Creek (upper) } \\
\hline 1990 & 27 April & 30 May & 22 June & 17 April - 18 July \\
\hline 1991 & 18 May & 10 June & 26 June & 26 April - 01 July \\
\hline 1992 & 22 April & 08 May & 03 June & 15 April - 26 June \\
\hline 1993 & 08 May & 18 May & 26 May & 26 April - 15 Junk \\
\hline 1994 & 03 May & 19 May & 19 July & 25 April - 30 August \\
\hline 1995 & 05 May & 23 May & 09 June & 02 May - 26 June \\
\hline \multicolumn{5}{|c|}{ Big Creek (lower)/Rush Creek } \\
\hline 1993 & 24 April & 29 April & 13 May & 21 April - 16 May \\
\hline 1994 & 23 April & 29 April & 11 May & 21 April - 15 June \\
\hline 1995 & 19 April & 01 May & 14 May & 11 April - 05 June \\
\hline \multicolumn{5}{|c|}{ West Fork Chamberlain Creek } \\
\hline $1992 "$ & 15 April & 26 April & 03 June & 12 April - 24 June \\
\hline 1993 & 28 April & 15 May & 23 June & 23 April - 22 July \\
\hline $1994 "$ & 24 April & 01 May & 05 July & 24 April - 04 September \\
\hline $1995^{c}$ & 16 April & 09 May & 20 June & 12 April - 22 September \\
\hline \multicolumn{5}{|c|}{ Secesh River } \\
\hline 1989 & 20 April & 27 April & 09 June & 09 April - 19 July \\
\hline 1990 & 14 April & 22 April & 07 June & 10 April - 13 July \\
\hline 1991 & 20 April & 27 April & 14 June & 13 April - 20 July \\
\hline 1992 & 13 April & 29 April & 04 June & 05 April - 03 July \\
\hline 1993 & 26 April & 16 May & 16 June & 22 April - 15 July \\
\hline 1994 & 22 April & 26 April & 11 July & 21 April - 07 August \\
\hline 1995 & 14 April & Ol May & 24 May & 10 April - 10 July \\
\hline
\end{tabular}


Table 6. Continued.

\begin{tabular}{|c|c|c|c|c|}
\hline \multirow[b]{2}{*}{ Year } & \multicolumn{4}{|c|}{ Passage dates at Lower Granite Dam } \\
\hline & $10 \%$ & $\mathbf{5 0 \%}$ & $90 \%$ & $\mathrm{R}$ a n g e \\
\hline \multicolumn{5}{|c|}{ Lake Creek } \\
\hline 1989 & 23 April & 02 May & 16 June & 12 April - 01 July \\
\hline $1990^{\mathrm{b}}$ & & -------- & ---- & $\longrightarrow$ \\
\hline $1991^{b}$ & & $--w-w---$ & ---W---- & - \\
\hline $1992^{b}$ & & ------- & $-\mathrm{e}^{----}$ & \\
\hline 1993 & 23 April & 09 May & 22 June & 22 April - 25 June \\
\hline 1994 & 21 April & 28 April & 19 May & 20 April - 24 June \\
\hline 1995 & 17 April & 10 May & 10 June & 14 April - 20 July \\
\hline \multicolumn{5}{|c|}{ Catherine Creek } \\
\hline 1991 & 01 May & 14 May & 08 June & 17 April - 23 June \\
\hline 1992 & 16 April & 01 May & 21 May & 09 April - 29 June \\
\hline 1993 & 06 May & 18 May & 05 June & 29 April - 26 June \\
\hline 1994 & 25 April & 11 May & 20 May & 13 April - 26 July \\
\hline 1995 & 01 May & 19 May & 09 June & 26 April - 02 July \\
\hline \multicolumn{5}{|c|}{ Grande Ronde River (upper) } \\
\hline 1989 & 12 May & 06 June & 19 June & 27 April - 22 July \\
\hline $1990^{b}$ & ------- & ---m--e- & ---- & --e m----.- \\
\hline $1991^{b}$ & ------- & ----m-e- & $---\mathrm{e}$ & $\longrightarrow$ \\
\hline $1992^{b}$ & -------- & ------- & $w^{-} c^{--}$ & ---------------- \\
\hline 1993 & 05 May & 16 May & 25 May & 23 April - 20 June \\
\hline 1994 & 28 April & 23 May & 07 July & 23 April - 29 August \\
\hline 1995 & 27 April & 29 May & 12 June & 12 April - 01 July \\
\hline
\end{tabular}


Table 6. Continued.

\begin{tabular}{|c|c|c|c|c|}
\hline \multirow[b]{2}{*}{ Year } & \multicolumn{4}{|c|}{ Passage dates at Lower Granite Dam } \\
\hline & $10 \%$ & $50 \%$ & $90 \%$ & Range \\
\hline \multicolumn{5}{|c|}{ Imnaha River (lower) } \\
\hline 1989 & 11 April & 30 April & 11 May & 04 April - 05 June \\
\hline 1990 & 10 April & 18 April & 09 May & 05 April - 27 May \\
\hline 1991 & 20 April & 01 May & 13 May & 14 April - 15 May \\
\hline 1992 & 10 April & 21 April & 03 May & 06 April - 21 May \\
\hline $1993^{b}$ & & $\ldots .$. & ------ & --------------- \\
\hline $1994^{b}$ & & -------- & $\cdots$ & $-\ldots-\ldots$ \\
\hline $1995^{b}$ & & & & \\
\hline \multicolumn{5}{|c|}{ Imnaha River (upper) } \\
\hline 1993 & 24 April & 14 May & 28 May & 15 April - 23 June \\
\hline 1994 & 24 April & 08 May & 09 June & 20 April - 11 August \\
\hline 1995 & 13 April & 02 May & 03 June & 10 April $07 \mathrm{~J}$ u 1 y \\
\hline \multicolumn{5}{|c|}{ Lostine River } \\
\hline $1990 "$ & $\longrightarrow$ & -ב---- & & \\
\hline 1991 & 29 April & 14 May & 26 May & 20 April -09 July \\
\hline 1992 & 16 April & 30 April & 11 May & 12 April - 02 June \\
\hline 1993 & 23 April & 03 May & '17 May & 17 April $\theta 1$ June \\
\hline 1994 & 22 April & 30 April & 16 May & 19April-07 June \\
\hline 1995 & 12 April & 02 May & 17May & OS April - 09 June \\
\hline
\end{tabular}

a Insufficient numbers detected to estimate timing.

$b$ No fish were tagged for this migration year.

c Includes fish from Chamberlain Creek. 
for fish from these streams were distributed from early to late-May (Appendix Tables 4A, 5A, 7A, 8A, 1OA, 11A, 15A, 18A, 19A, 21A, and Fig. 4).

Fish from Big Creek (upper) and Sulphur Creek in the Middle Fork of the Salmon River drainage, Valley Creek in the upper Salmon River, and the Grande Ronde River (upper) in Oregon had the latest passage periods at Lower Granite Dam (Fig. 4 and Table 6). The 50\% passage dates for fish from these streams ranged from 23 May to 2 June. Peak passage dates for fish from these streams occurred over an extended period from early May to late June (Appendix Tables 6A, 9A, 14A, and Fig. 4). Fish from both Valley and West Fork Chamberlain/Chamberlain Creeks had the most protracted passage period at the dam. Valley Creek fish had the latest passage period of all streams at the dam.

Passage distributions for wild chinook salmon smolts from Idaho streams were quite variable at Lower Granite Dam in 1995. Analyses of passage distributions were potentially biased due to low numbers and the spill program, even though detection numbers were. adjusted for spill. We used the Student-Newmann-Keuls (SNK) multiple comparison method to make between-stream comparisons for each statistic. Oregon streams were not included in this analysis. There were many significant $(\mathrm{P}<0.05)$ timing differences for the 10,50 , and $90 \%$ passage distributions at the dam between streams using the median of the individual stream bootstrap standard errors. Overall, for the middle $80 \%$ passage time in days at the dam, Lake Creek and West Fork Chamberlain/Chamberlain Creeks fish had significantly longer passage times than Big (lower)/Rush Creeks, East Fork of the Salmon River, or Herd Creek fish $(\mathrm{P}<0.05)$. Valley Creek fish had significantly longer passage time at the dam than Big (lower)/Rush Creeks, East Fork of the Salmon River, Herd Creek, Loon Creek, 
Marsh Creek, Big Creek (upper), Sulphur Creek, Camas Creek, or the Secesh River fish $(\mathrm{P}<0.05)$.

Timing of smolts from individual streams in Idaho is not presented here for Little Goose, Lower Monumental, McNary, John Day, and Bonneville Dams. See Appendix Tables 4A21B for this information.

We combined all detections of wild fish from Idaho streams at each of the four collector dams and compared the timing at each dam with river flows during the same periods (Fig. 5). Overall passage occurred between early April and late September at Lower Granite Dam, with the middle $80 \%$ passage from late April to early June (Table 7). The peak passage date was 9 May, which coincided with an increasing flow period, but prior to peak flows at this dam in early June (Appendix Table 23). The middle $80 \%$ passage of wild fish occurred between early May and the first two weeks of June for Little Goose, Lower Monumental, and McNary Dams (Table 7). Peak passage periods for fish at Little Goose, Lower Monumental, and McNary Dams coincided-with moderate to high river flows during mid-May; these peaks occurred prior to peak flows at these dams, which extended from late May to early June (Fig. 5 and Appendix Tables 24-26).

\section{DIEL TIMING AT JUVENILE FISH FACILITIES}

Diel timings at collector dams were based on detections of fish exiting the fish and debris separators at the juvenile fish facilities. Timing was calculated by totaling detections of combined populations of wild fish for each of the 24 daily hours through the migration and dividing by the total detected for the migration (Fig. 6). 
Table 7. Passage-dates at Lower Granite, Little Goose, Lower Monumental, and McNary Dams for combined populations of PIT-tagged wild spring/summer chinook salmon smolts from Idaho in 1995.

\begin{tabular}{lcccc}
\hline \multirow{2}{*}{ Site } & \multicolumn{4}{c}{ Passage periods at dams } \\
\cline { 2 - 5 } & $10 \%$ & $50 \%$ & $90 \%$ & Range \\
\hline \hline Lower Granite Dam & 19 April & 10 May & 6 June & 10 April - 22 September \\
Little Goose Dam & 1 May & 14 May & 11 June & 13 April - 7 September \\
Lower Monumental Dam & 3 May & 16 May & 11 June & 19 April - 27 August \\
McNary Dam & 2 May & 18 May & 6 June & 28 April - 2 July \\
\hline
\end{tabular}



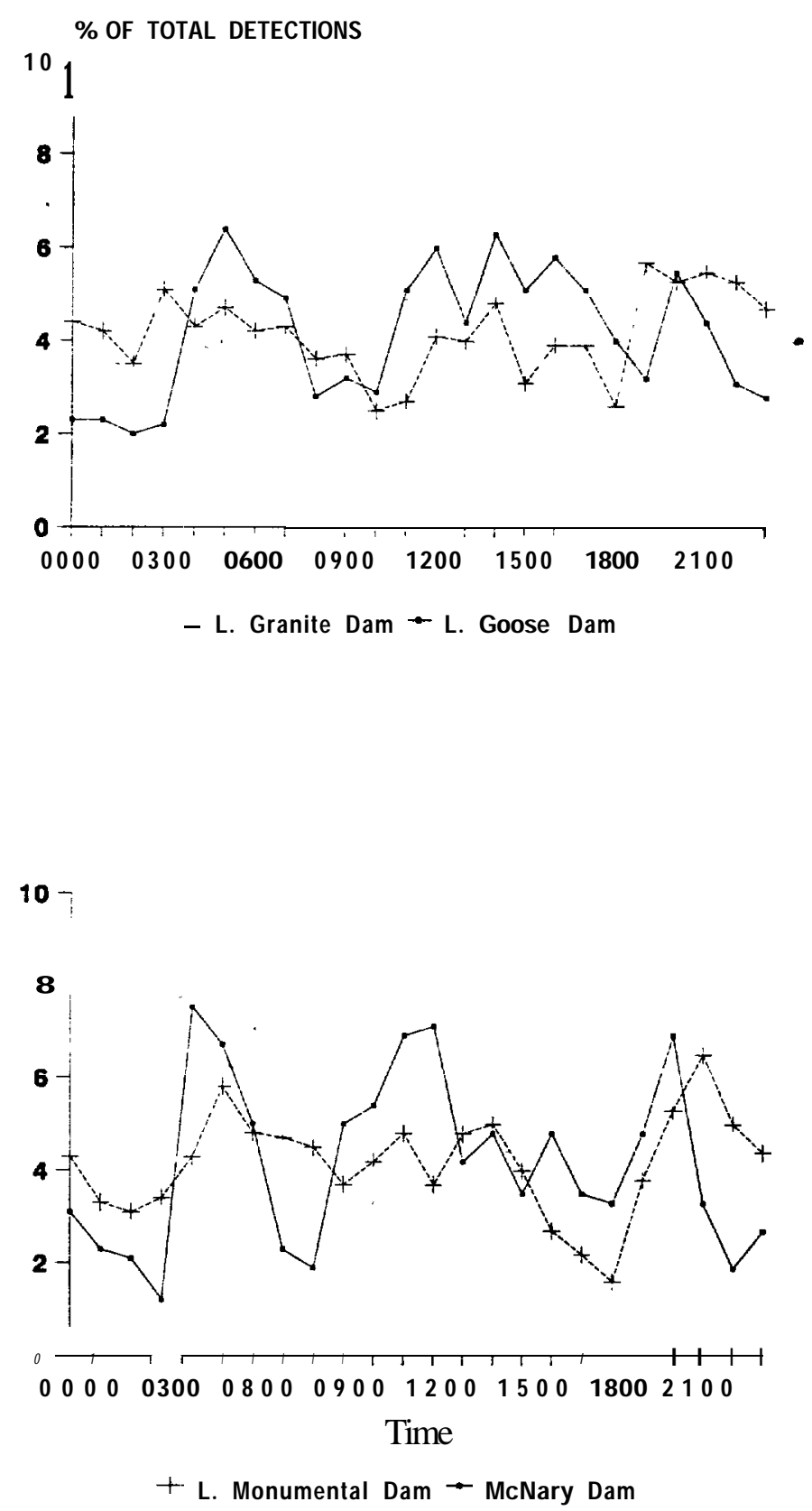

Figure 6. Diel passage timing of all PIT-tagged wild chinook salmon smolts from Idaho at Lower Granite, Little Goose, Lower Monumental, and McNary Dams in 1995. Diel timing was calculated on an hourly basis for fish exiting the fish and debris separators at the dams during the migration. 
At Lower Granite Dam, significantly more wild smolts exited the separator from 1800 to $0600 \mathrm{~h}$ (mostly nighttime hours, 55.2\%) than exited from 0600 to $1800 \mathrm{~h}$ (daytime hours, 44.8\%) $(\mathrm{P}<0.0007)$. When we examined passage in 6-hour periods, the lowest numbers of fish exited the separator from 0600 to $1200 \mathrm{~h}$, and the highest numbers exited from 1800 to 0000 h. Using chi-square tests, we found significantly fewer fish detected from 0600 to $1200 \mathrm{~h}$ than from 0000 to $0600 \mathrm{~h}$ or from 1800 to $0000 \mathrm{~h}$, and significantly fewer fish were detected from 1200 to $1800 \mathrm{~h}$ than from 1800 to $0000 \mathrm{~h}(\mathrm{P}<0.002)$. Peak passage from the separator occurred in early morning and evening.

At Little Goose Dam, significantly more wild smolts exited the fish and debris separator from 0600 to $1800 \mathrm{~h}(56.7 \%)$ than exited from 1800 to $0600 \mathrm{~h}(43.3 \%)$ ( $<<0.0002)$. The lowest numbers of fish exited the separator from 0000 to $0600 \mathrm{~h}$, and the highest numbers exited from 1200 to $1800 \mathrm{~h}$. Significantly more fish were detected from 1200 to $1800 \mathrm{~h}$ than for the other three 6-hour time periods $(\mathrm{P}<0.0001)$. Peak passage times were early morning and mid-afternoon.

At Lower Monumental Dam, although more wild smolts exited the fish and debris separator from 1800 to $0600 \mathrm{~h}(50.7 \%)$ than exited from 0600 to $1800 \mathrm{~h}(49.3 \%)$, the difference was not significant $(\mathrm{P}>0.6)$. The lowest numbers of fish exited from 1200 to $1800 \mathrm{~h}$ and the highest numbers exited from 0600 to $1200 \mathrm{~h}$; however, there were no significant differences between the four time periods $(\mathrm{P}>0.2)$. The major peak passage period was from 2100 to $2200 \mathrm{~h}$.

At McNary Dam, more wild smolts exited the fish and debris separator from 0600 to $1800 \mathrm{~h}(54.3 \%)$ than exited from 1800 to $0600 \mathrm{~h}(45.7 \%)$, but the difference was not 
significant $(\mathrm{P}>0.05)$. Equally low numbers of fish exited from 1800 to $0000 \mathrm{~h}$ and 0000 to $0600 \mathrm{~h}$. The highest numbers exited from 1200 to $1800 \mathrm{~h}$. There were no significant differences between the four time periods $(\mathrm{P}>0.2)$. Peak passage periods occurred in early morning, midday, and evening.

\section{ENVIRONMENTAL INFORMATION}

One goal of this study is to identify relationships between environmental factors where wild parr reside and subsequent migration timing of smolts the following spring at downstream traps and dams. Since 1993, NMFS has worked with Pacific Northwest Laboratories (PNL) through Bonneville Power Administration funding to obtain environmental data.

In 1993, PNL personnel conducted an extensive review of historical and current environmental information collected in Idaho study streams. In November and December 1993, they installed environmental monitoring systems in the Middle Fork of the Salmon River near Thomas Creek, Marsh Creek, Valley Creek, the upper Salmon River near Sawtooth Hatchery, and the Salmon River below its confluence with the Yankee Fork. Monitors will be installed in other study streams during the next few years. Achord et al. (1995b) provided additional information about stream monitors.

Appendix Tables 27-3 1 provide a summary of environmental information collected at these 5 sites from August 1994 to July 1995. 


\section{ADULT RETURNS FROM 1989-1993 SMOLT MIGRATIONS}

Although adult return information is not an objective of this study, there is considerable interest concerning the return of PIT-tagged adult wild spring/summer chinook salmon to the Snake River. Of the wild spring/summer chinook salmon PIT tagged and released for the 1989 through 1993 smolt migrations, 20 were detected as adults at the adult trap at Lower Granite Dam through 1995. Of the 20 adults, 12 had been transported as smolts from Lower Granite Dam to below Bonneville Dam, 4 had been transported as smolts from Little Goose Dam to below Bonneville Dam, and 4 had never been detected at any dam during previous smolt migrations.

\section{DISCUSSION}

Mortalities associated with collection and tagging in 1994 were comparable to those in earlier years (Matthews et al. 1990, 1992; Achord et al. 1992, 1994, 1995a, 1995b).

With the addition of juvenile migrant fish traps on Marsh Creek and South Fork of the Salmon River, more PIT-tagged wild fish were monitored at traps than in previous years. At Marsh Creek trap; where the vast majority of PIT-tagged wild fish were monitored (at traps), we compared the overall detection rate (unadjusted) at the dams for released fish in the summer $(10.7 \%)$, to that of fish detected and released from the trap in fall $(18.8 \%)$. This $75.7 \%$ increase in detection rate at the dams for released previously PIT-tagged fish detected at traps in the fall may indicate a higher survival for known fall migrants and/or reflect mortality in the stream over an average of 51 days from summer to fall in 1994. Kiefer and Lockhart (1993 and 1994) observed a 100\% increase in overall detection rates at the dams the 
following springs for PIT-tagged chinook salmon emigrating from the upper Salmon River in fall 1990 and 1991 (9.5 and 7.1\%), compared to the overall detection rates at the dams for chinook salmon parr PIT tagged in summer 1990 and 1991 (4.7 and 3.5\%).

For data collected over the last 7 years, length-distribution curves showed that, generally, wild fish released and subsequently detected at dams are slightly larger than fish that are released but not detected. The reason for this slight difference in size is unknown. However, it appears that larger fish, tagged and released the previous summers, survived slightly better and/or were guided slightly better into the collection systems at the dams than smaller fish.

Another consistent trend we "have observed over the years is the difference in migration timing at dams with respect to size at tagging. Wild fish migrating in April and May were . significantly larger at release than fish migrating after May. This consistent trend suggests that size is an important factor related to either the initiation of smoltification or other lifehistory dynamics that affect the migrational timing of wild fish.

In 1995, the overall detection rate (adjusted) of wild fish at the four collector dams was lower than in 1993 (Achord et al. 1995a) or 1994 (Achord et al. 1995b). Densities of chinook salmon parr in all streams in 1994 were the highest since 1989. For example, in 1994, we collected 2.6 times more fish than in 1993 while sampling $29.6 \%$ less stream distance. In 1994 density-dependant effects may have contributed to the lower overall average detection rate at the dams in 1995. However, other factors, such as changes in spill patterns and fish guiding efficiencies (installation of some extended-length turbine intake screens), may have contributed to the difference in dam detection rates between the 2 years. 
As observed in 1993 and 1994, fish from Big (lower)/Rush Creeks had the highest detection rate at the dams in 1995 .

Average gains in fork length and weight observed for wild chinook salmon from time of release to recovery at McNary Dam, were similar to those observed at Lower Monumental, Little Goose, and Lower Granite Dams during previous studies (Matthews et al. 1992; Achord et al. 1992, 1995a, 1995b). The average length gain for, the eight wild fish measured at John * Day and Bonneville Dams was similar to those measured at McNary Dam.

In 1995, as in 1992, 1993, and 1994, protracted passage distributions and small sample sizes at Lower Granite Dam made it difficult to statistically quantify small differences in arrival timing among fish from different streams. Since the timing of fish from all Idaho streams (excluding Oregon streams) tended to be early and very protracted at the dam in 1992, there were no statistically significant timing differences among streams for that year. Timing of fish from most Idaho streams was late in 1993, with more fish passing the dam during a shorter period, so it was possible to detect some significant timing differences among fish from different streams in that year. In 1994, the middle $80 \%$ arrival timing distributions of fish from Idaho streams were quite variable, ranging from 18 to 80 days; therefore, some significant timing differences were detected. In 1995, the middle $80 \%$ arrival timing distributions of fish from Idaho streams were also quite variable, ranging from 25 to 65 days, and several significant timing differences 'were observed. Fish from the East Fork of the Salmon River had the earliest passage distribution, while Valley Creek fish displayed the latest passage distribution at the dam. Table 6 shows the variability in passage dates at Lower Granite Dam for individual streams in Idaho and Oregon over the years. 
In 1995, peak detections of wild fish occurred on 9 May, and did not coincide with peak river flows at Lower Granite Dam. However, the peak passage period around 9 May. at the dam did coincide with increasing river flows. This period of medium-to-high flows was followed by high flows throughout the remaining month of May, with flows peaking in early June at the dam. In the 6 years before 1995, passage timing of wild smolts at this dam has been highly variable and generally independent of river flows before mid-May (Fig. 7). In contrast, peak passage of wild fish after mid-May has coincided well with periods of peak river flow at this dam before 1995. It appears the sustained high flows from mid-May to early June moved wild fish through this dam in a more uniform fashion than in previous years, and by the time peak flows occurred on 6 June, $90 \%$ had passed the dam. Raymond (1979) showed that the peaks of migration for the composite population of spring and summer chinook salmon smolts (mostly wild) passing Ice Harbor Dam from 1964-1969 preceded the periods of maximum river discharge in most years. During these years, fish passage peaked between 26 April and '13 May. With respect to river flow, our observations match the results of Raymond for wild fish migrating before mid-May.

Annual overall climatic variation is emerging as an important factor controlling overall timing of wild spring/summer chinook salmon smolts at Lower Granite Dam. Figures 7 and 8 give a perspective on timing of combined populations (Idaho and Oregon) of wild spring/summer chinook salmon smolts from 1989 through 1995 at this dam. In the colderthan-normal years of 1989, 1991, and 1993, 50\% of all wild fish had passed the dam by midMay, while $90 \%$ passed by mid-June (except in 1993, when high flows moved 90\% through the dam by the end of May). In the warmer-than-normal years of 1990, 1992, and 1994, 50\% 

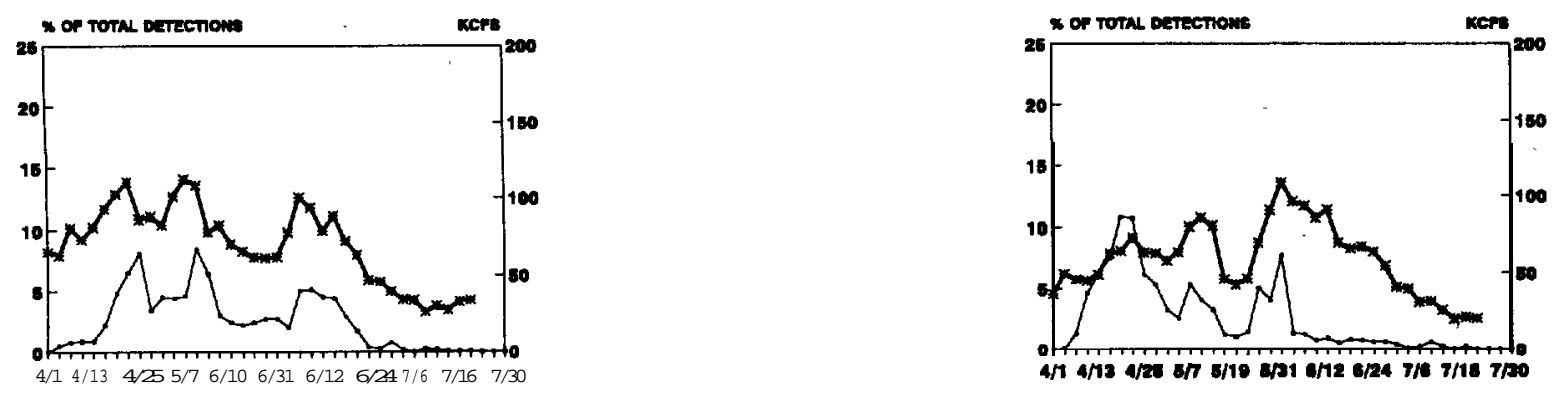

1991

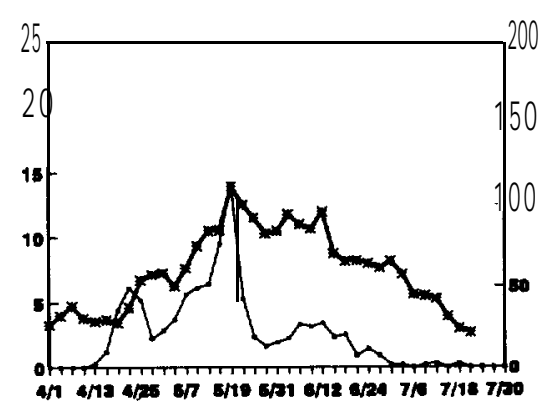

1992

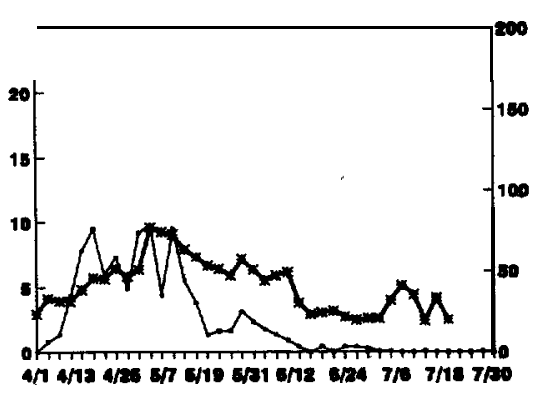

1993

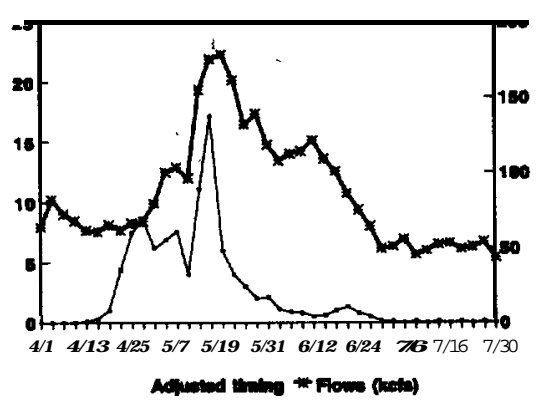

1994

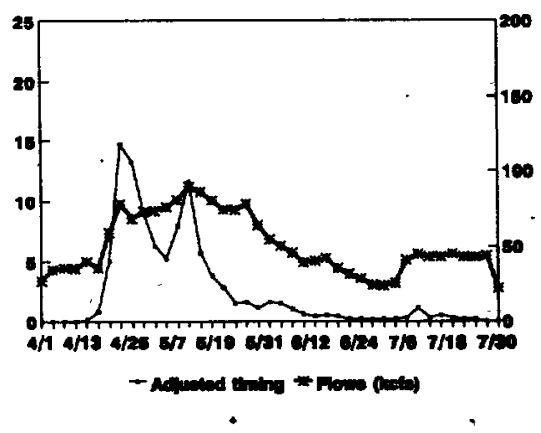

Figure 7. A historical perspective of migration timing (adjusted in spill years) of wild spring/summer chinook salmon smolts at Lower Granite Dam 1989-1995, with associated river flows at the dam. Data represent PIT-tag detections from Idaho and Oregon streams combined by 3-day intervals and average river flows at the dam over the same time periods. 


\section{5}

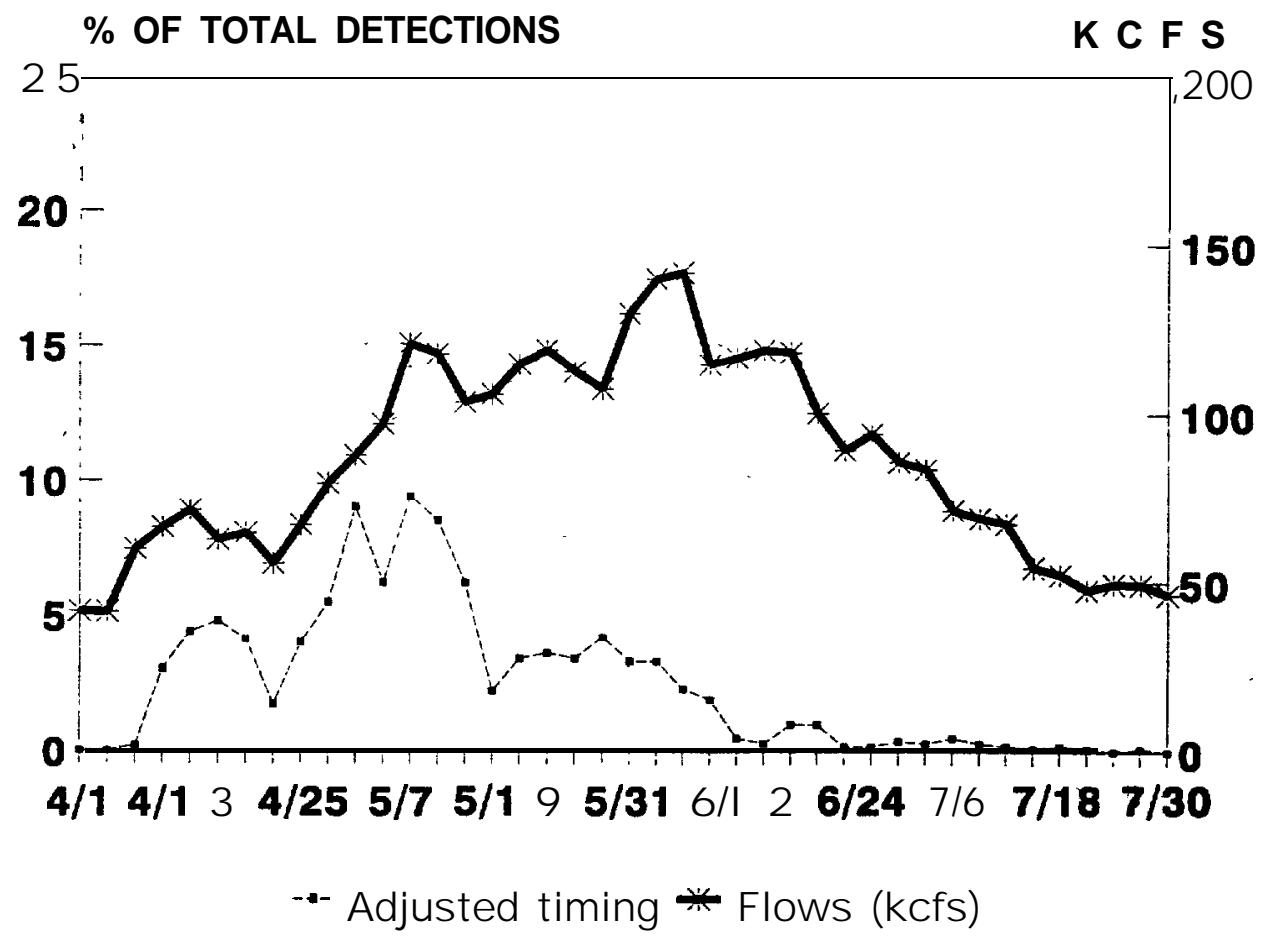

Figure 7. Continued. 


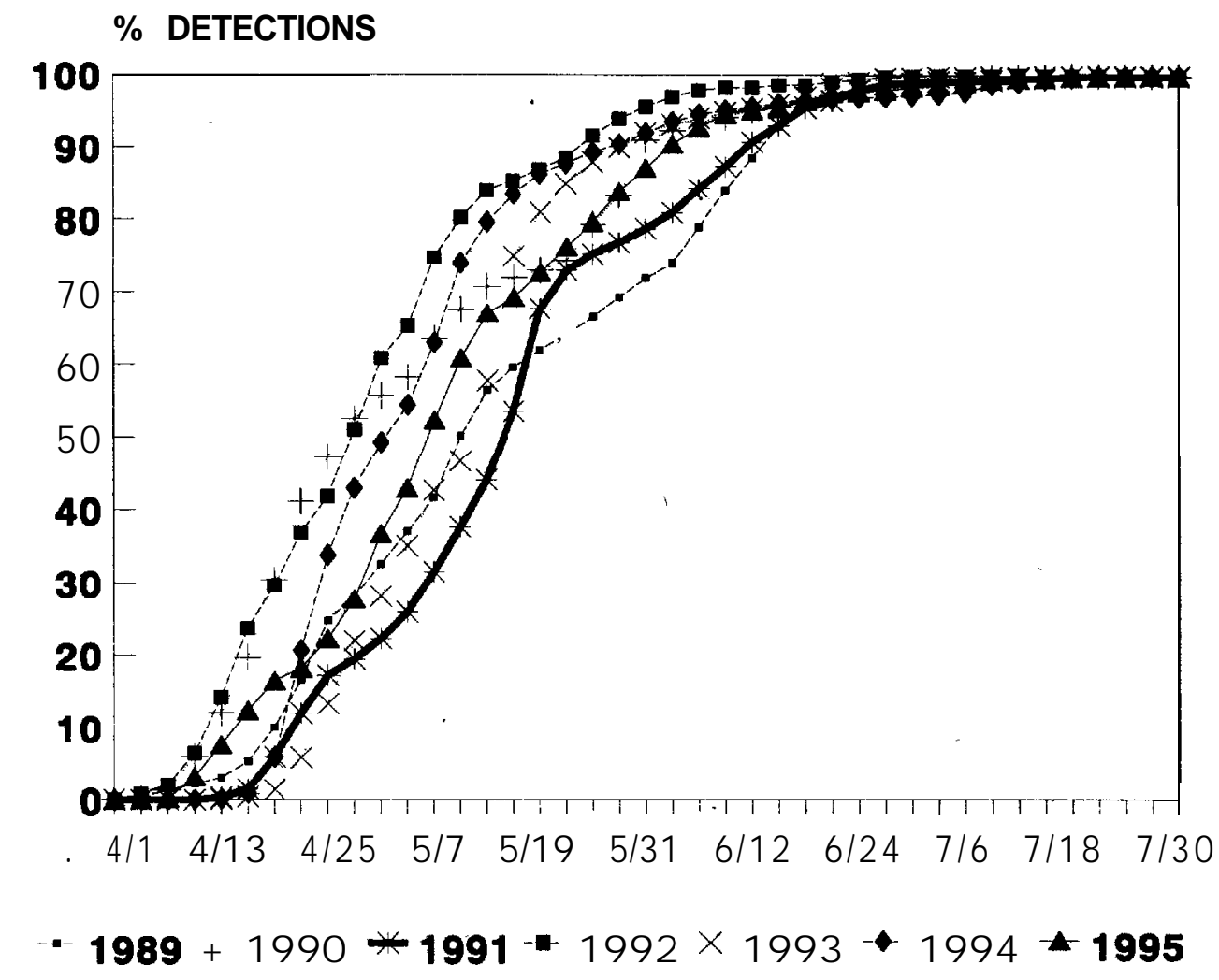

Figure 8. Cumulative percentages of total detections (adjusted for spill) of PIT-tagged wild spring/summer chinook salmon smolts detected at Lower Granite Dam, 1989-1995. Data represent PIT-tag detections from Idaho and Oregon streams combined by 3day intervals. 
of all wild fish passed this dam from 29 April to 4 May, and 90\% passed by the end of May. Over these 6 years, we see a consistent 2-week shift in timing of wild fish at this dam between relatively warm and relatively cold years. In 1995, we see intermediate passage dates of 9 May and 5 June for the 50 and $90 \%$ passage dates, respectively, for these combined wild populations (Fig. 8). In 1995, we experienced near-normal weather conditions in late winter and early spring. Raymond (1979) cited water temperature as one of the most important factors for triggering the downstream movements of hatchery-reared and wild chinook salmon smolts in spring. He also noted that the latest migrations occurred in years when runoff was delayed by cold weather.

In 1992 and 1993, peak detections of wild fish at the collector dams below Lower Granite Dam coincided well with peak river flows. We were unable to determine whether the increased river flow moved these groups of fish through the reservoirs or were simply coincidental with their arrival at the dams. Since peak detections at these dams have consistently occurred nearly simultaneously with increased flow, it seemed likely that the fish were near the dams and were moved through them rapidly by the increased flow. However, this did not apparently occur in 1994. Peak detections at the lower collector dams did not coincide well with peak flows. In fact, peak flows at these dams coincided with significant decreases in wild fish detections, even though detections were adjusted for spill. We found no explanation for this difference. In 1995, peak detections of wild fish at these dams coincided with medium to high flows prior to peak flows.

After examining chinook salmon smolt passage timing at the dams over the last 7 years, it has become clear that flow is only one of several factors that influences passage timing. 
Other factors. such as water temperature. turbidity. physiological development. variability in stock behavior. fish size. and other yet unknown conditions may equally affect wild smolt passage timing at dams.

In 1995. as was observed in 1992. 1993. and 1994, diel timings of wild fish exiting the fish and debris separators during the migration season varied among dams. In 1992. slightly more wild fish exited the separator at Lower Granite Dam during daytime hours. but in 1993. 1994. and 1995. significantly more wild fish exited the separator during mostly nighttime hours. At Little Goose Dam, the diel timing in 1995 was similar to timing observed in 1992 and 1993. with more wild fish exiting the separator during daytime hours. We also observed more wild fish exiting the separator during daytime hours in 1994, but the proportion was larger than in the other 3 years. At Louer Monumental Dam. more wild fish exited the separator during mostly nighttime hours in 1994 and 1995. but the difference was significant only in 1994, At McNary Dam. more wild fish exited the separator during daytime hours in all 4 years: however. in 1992 and 1994. a larger proportion exited the separator during daytime hours than in 1993 and 1995. We offer no explanation for diel timing differences observed at the dam separators over the years. 


\section{ACKNOWLEDGMENTS}

We thank Neil N. Paasch. Kenneth W. McIntyre. Eric E. Hockersmith, Paul A. Ocker.

Leslie K. Timme. and others of our staff who participated in collecting and PIT tagging the thousands of fish involved in this study. We also thank Brad Gilmore of Pacific Northwest Laboratories for providing environmental information included in this report. 


\section{REFERENCES}

Achord. S.. J. R. Harmon. D. M. Marsh, B. P. Sandford. K. W. McIntyre. K. L. Thomas, N. N. Paasch. and G. M. Matthews. 1992. Research related to transportation of juvenile salmonids on the Columbia and Snake Rivers, 1991. Report to U.S. Army Corps of Engineers. Contract DACW68-84-H0034. 57 p. plus Appendix. (Available from Northwest Fisheries Science Center. 2725 Montlake Blvd. E., Seattle, WA 981122097.)

Achord. S.. D. J. Kamikawa. B. P. Sandford. and G. M. Matthews. 1995a. Monitoring the migrations of wild Snake River spring/summer chinook salmon smolts, 1993. Report to Bonneville Power Administration. Project 91-028. Contract DE-A179-91 BP1 8800. 88 p. (Available from Northwest Fisheries Science Center. 2725 Montlake Blvd. E., Seattle. WA 98112-2097.)

Achord, S., D. J. Kamikawa. B. P. Sandford, and G. M. Matthews. 1995b. Monitoring the migrations of wild Snake River spring/summer chinook salmon smolts. 1994. Report to Bonneville Power Administration. Project 91-028, Contract DE-AI79-91BPI 8800. 100 p. (Available from Northwest Fisheries Science Center. 2725 Montlake Blvd. E.. Seattle. WA. 98112-2097.)

Achord. S.. G. M. Matthews, D. M. Marsh. B. P. Sandford. and D. J. Kamikawa. 1994. Monitoring the migrations of wild Snake River spring and summer chinook salmon smolts. 1992. Report to Bonneville Power Administration. Project 91-28. Contract DE-AI79-9 1 BP 18800. 73 p. (Available from Northwest Fisheries Science Center, 2725 Montlake Blvd. E.. Seattle. WA 98112-2097.)

Kiefer. R. B. and J. N. Lockhart.

1993. Idaho habitat \& natural production monitoring: Part II. Report to Bonneville Power Administration, Project 91-73, Contract DE-BI79-91BP21182. 66 p. (Available from Bonneville Power Administration. Division of Fish and Wildlife. P.O. Box 3621. Portland. OR. 97283-3621.)

Kiefer. R. B. and J. N. Lockhart.

1994. Intensive evaluation and monitoring of chinook salmon and steelhead trout production. Crooked River and upper Salmon River sites. Report to Bonneville Power Administration. Project 9 I-73. Contract DE-BI79-91 BP21 182. 69 p. (Available from Bonneville Power Administration. Division of Fish and Wildlife. P.0. Box 3621, Portland. OR. 97283-3621.) 
Matthews. G. M.. S. Achord. J. R. Harmon. 0. W. Johnson. D. M. Marsh. B. P. Sandford. N. N. Paasch. K. W. McIntyre. and K. L. Thomas. 1992. Evaluation of transportation of juvenile salmonids and related research on the Columbia and Snake Rivers. 1990. Report to U.S. Army Corps of Engineers. Contract DACW68-84-H0034. 51 p. plus Appendix. (Available from Northwest Fisheries Science Center. 2725 Montlake Blvd. E.. Seattle WA 9811 Z-2097.)

Matthews. G. M.. J. R. Harmon. S. Achord. 0. W. Johnson. and L. A. Kubin. 1990. Evaluation of transportation of juvenile salmonids and related research on the Snake and Columbia Rivers. 1989. Report to U.S. Army Corp of Engineers. Contract DACW68-84-H0034. 59 p. plus Appendix. (Available from Northwest Fisheries Science Center. 272.5 Montlake Blvd. E.. Seattle WA 98112-2097.)

Pacific Northwest Electric Power Planning Council and Conservation Act. 1980. Columbia River Basin Fish and Wildlife Program. Northwest Power Planning Council. Portland. OR.

Prentice. E. F.. T. A. Flagg. and C. S. McCutcheon. 1990. PIT-tag monitoring systems for hydroelectric dams and fish hatcheries. Am. Fish. Soc. Symp. 7323-334.

Raymond. H. L. 1979. Effects of dams and impoundments on migrations of juvenile chinook salmon and steelhead from the Snake River. 1966 to 1975. Trans. Am. Fish. Soc. 108(6):505-529. 
APPENDIX TABLES 
Appendix Table 1. Summary of tagging dates, number collected, tagged, released, and minimum, maximum, and average lengths and weights of wild chinook salmon parr PIT tagged in various Idaho streams in 1994.

\begin{tabular}{|c|c|c|c|c|c|c|c|c|c|c|c|c|c|}
\hline \multirow[b]{2}{*}{ Stream } & \multirow{2}{*}{\multicolumn{2}{|c|}{ Tagging }} & \multirow{2}{*}{\multicolumn{2}{|c|}{ dates }} & \multirow{3}{*}{$\begin{array}{c}\begin{array}{c}\text { Number } \\
\text { collected }\end{array} \\
1,558\end{array}$} & \multirow{3}{*}{$\begin{array}{r}\begin{array}{r}\text { Number } \\
\text { tagged }\end{array} \\
1,460\end{array}$} & \multirow{3}{*}{$\begin{array}{c}\begin{array}{c}\text { Number } \\
\text { released }\end{array} \\
\mathbf{1 , 4 5 5}\end{array}$} & \multicolumn{3}{|c|}{ Length $(\mathbf{m m})$} & \multicolumn{3}{|c|}{ Weight (9) } \\
\hline & & & & & & & & \multirow{2}{*}{$\begin{array}{l}\text { Range } \\
\text { 49- }\end{array}$} & \multirow{2}{*}{\multicolumn{2}{|c|}{$\begin{array}{lc} & \text { Average } \\
82 & 63.1\end{array}$}} & \multicolumn{2}{|c|}{ Range } & \multirow{2}{*}{$\frac{\text { Average }}{3.5}$} \\
\hline Bear Valley Creek & 27 & Jul & -29 & Jul & & & & & & & 1.6 & 7.7 & \\
\hline Elk Creek & 29 & Jul & -01 & Aug & 1,542 & 1,514 & 1,512 & $51-$ & 85 & 66.7 & 2.3 & 8.2 & 4.1 \\
\hline Sulphur Creek & 31 & Jul & -01 & Aug & 769 & 728 & 728 & $52-$ & 85 & 62.0 & 1.4 & 6.7 & 3.0 \\
\hline Marsh Creek & & 02 & Aug & & 1,680 & 1,590 & 1,575 & $49-$ & 118 & 69.0 & 1.3 & 8.5 & 4.0 \\
\hline Cape Horn Creek & & 03 & Aug & & 3,833 & 1,445 & 1,443 & $47-$ & 100 & 61.7 & 1.4 & 5.0 & 2.7 \\
\hline Valley Creek & 04 & Aug & -05 & Aug & 1,944 & 1,552 & 1,552 & $51-$ & 109 & 64.2 & 2.2 & 6.6 & 3.7 \\
\hline Camas Creek & 08 & Aug & -10 & Aug & 1,986 & 1,534 & 1,528 & $49-$ & 86 & 61.5 & 1.6 & 7.1 & 3.2 \\
\hline Loon Creek & & 09 & Aug & & 1,023 & 964 & 964 & $50-$ & 84 & 64.6 & 1.7 & 6.7 & 3.5 \\
\hline Herd Creek & 12 & Aug & -13 & Aug & 568 & 534 & 534 & $53-$ & 99 & 73.7 & 3.9 & 3.9 & 3.9 \\
\hline E. F. Salmon River & 12 & Aug & -13 & Aug & 1,070 & 986 & 986 & $50-$ & 96 & 73.8 & 1.8 & -11.8 & 5.4 \\
\hline Big Creek (upper) & & 17 & Aug & & 862 & 757 & 757 & $45-$ & 82 & 62.1 & 1.7 & 6.7 & 3.4 \\
\hline S. F. Salmon River & 16 & Aug & -18 & Aug & 4,017 & 1,574 & 1,571 & $40-$ & 94 & 59.0 & 1.3 & 8.5 & 2.6 \\
\hline Big Creek (lower) & & 21 & Aug & & 757 & 727 & 727 & $53-$ & 91 & 75.2 & 2.8 & 7.4 & 5.3 \\
\hline Rush Creek & & 21 & Aug & & 15 & 15 & 15 & $69-$ & 93 & 74.9 & -- & --- & --- \\
\hline W F. Chamberlain & & 21 & Aug & & 928 & 917 & 917 & $52-$ & 97 & 66.2 & 1.6 & -11.0 & 3.3 \\
\hline Chamberlain Creek & & 22 & Aug & & 266 & 241 & 241 & 53 & 79 & 64.9 & --- & ---- & --- \\
\hline Secesh River & 23 & Aug & -24 & Aug & 1,593 & 1,551 & 1,551 & $49-$ & 97 & 62.9 & 1.2 & -12.0 & 3.2 \\
\hline Lake Creek & & 24 & Aug & & 463 & 406 & 405 & 51 & 99 & 62.9 & --- & ----- & --- \\
\hline Totals or averages & 27 & Jul & -24 & Aug & 24,074 & 18,495 & 18,462 & $45-$ & 118 & 65.0 & 1.2 & -12.0 & 3.6 \\
\hline
\end{tabular}


Appendix Table 2. A summary of observed collection mortality for PIT-tagged wild chinook salmon parr collected from streams in Idaho during July and August 1993.

\begin{tabular}{|c|c|c|c|c|c|c|c|}
\hline St-ream & $\begin{array}{l}\text { Collection } \\
\text { method }\end{array}$ & $\begin{array}{l}\text { Number } \\
\text { collected }\end{array}$ & $\begin{array}{l}\text { Number } \\
\text { tagged }\end{array}$ & $\begin{array}{l}\text { Number } \\
\text { rejected }\end{array}$ & $\begin{array}{l}\text { Percent } \\
\text { rejected }\left(\frac{\circ}{\circ}\right)\end{array}$ & $\frac{\text { Observed collection }}{\text { Mortality }}$ & $\frac{\text { mortality }}{\left(\frac{\circ}{0}\right)}$ \\
\hline Bear Valley Creek & seine $\bar{x}$ shcck & 1,558 & 1,450 & 71 & $4.6)$ & $2-t$ & 1.7 \\
\hline Elk Creek & seine \& shcck & 1,542 & 1,514 & 2 & C.1: & 26 & 17 \\
\hline Sulphur Creek & seine \& shock & 769 & 728 & 38 & ; 4.9$)$ & 3 & o. 4 \\
\hline Marsh Creek & seine \& shock & 1,680 & 1,590 & 96 & l 5. 1: & 4 & (0. 2' \\
\hline Cape $\because 33 n$ Creek & seize \& shock & 3,933 & 1,445 & 2,383 & (62. 2) & 5 & 0. 1 \\
\hline Valley Creek & seize \& shock & 1,444 & 1,552 & 357 & $\therefore-y \quad \|$ & 5 & 0.3 \\
\hline Camas Creek & seine a shock & 1,986 & i. 534 & 399 & i $23.1 \mathrm{i}$ & 53 & 2. 7 \\
\hline Loon Creek & shock & 1,023 & 964 & 37 & $: 3.6 j$ & 22 & (2. 2) \\
\hline Herd Creek & shcck & 568 & 534 & 7 & 2 & 27 & 4. 8 \\
\hline E. F. Salmon River & shock & 1,070 & 986 & 5 & 5; & 79 & $7 \cdot 4$ \\
\hline Big Creek (upper) & seine \& shcck & 962 & 757 & 33 & 10.8 & 12 & 1.4 \\
\hline S. F. Salmon River & seine \& shock & 4, 017 & 1,574 & 2,417 & 60.2 & 25 & 0.7 \\
\hline Big Creek (lower) & shock & 757 & 727 & 1 & 0. 1: & 29 & (3. 8) \\
\hline Rush Creek & shock & 15 & 15 & 0 & ---- & 0 & $\cdots--$ \\
\hline A F. Chamberlain Creek & seine & 428 & 417 & 11 & 1. 2 & 0 & $\cdots--$ \\
\hline Chamberlain Creek & shock & 266 & 241 & 24 & 9.0 & 1 & 0.4 \\
\hline Secesh Rive-- & shock & 1,593 & 1,551 & 33 & 2.1 & 9 & $(0.5)$ \\
\hline Lake Creek & shock & 463 & 406 & 56 & 12. 1 & 1 & (0.2) \\
\hline Tctals & & 24,874 & 19,495 & 6,050 & 24.3 & 323 & $1 \cdot 3$ \\
\hline
\end{tabular}


Appendix Table 3. A summary of observed post-tagging mortality and tag loss for PIT-tagged wild chinook salmon parr collected from streams in Idaho during July and August 1994.

\begin{tabular}{|c|c|c|c|c|c|c|c|c|c|}
\hline \multirow{2}{*}{ Stream } & \multirow{2}{*}{$\begin{array}{l}\text { Number } \\
\text { tagged }\end{array}$} & \multicolumn{5}{|c|}{ Observed post-tassins mortality } & \multirow{2}{*}{\multicolumn{2}{|c|}{24 hour tag loss }} & \multirow{2}{*}{$\begin{array}{l}\text { Number } \\
\text { releasec }\end{array}$} \\
\hline & & $\frac{1 / 2-3 h}{\text { Mortality }}$ & $\frac{\text { hours }}{10}$ & $\frac{24 \mathrm{hc}}{\mathrm{Numher} h e 1 \mathrm{~d}}$ & $\frac{12 s}{M a r t a l i t y}$ & $70^{\circ}$ & & & \\
\hline Bear Valley Creek & 1,460 & 3 & (0.2) & 448 & 2 & $(0.5)$ & 0 & $(0.0)$ & 1,455 \\
\hline Elk Creek & 1,514 & 2 & $(0.1)$ & 389 & 0 & $(0.0)$ & 0 & $(0.0)$ & 1,512 \\
\hline Sulphur Creek & 728 & 0 & $(0.0)$ & 75 & 0 & $(0.0)$ & 0 & $(0.0)$ & 728 \\
\hline Marsh Creek & 1,590 & 15 & $(0.9)$ & 128 & 0 & $(0.0)$ & 0 & $(0.0)$ & 1,575 \\
\hline Capehorn Creek & 1,445 & 0 & $(0.0)$ & 176 & 1 & ( 0.61 & 0 & $(0.0)$ & 1,443 \\
\hline Valley Creek & 1,552 & $\mathbf{0}$ & $(0.0)$ & 111 & 0 & $(0.0)$ & 0 & $(0.0)$ & 1,552 \\
\hline Camas Creek & 1,534 & 0 & $(0.0)$ & 812 & 6 & (0.7) & 0 & $(0.0)$ & 1,528 \\
\hline Loon Creek & 964 & 0 & $(0.0)$ & 0 & & $(---)$ & -- & $(--)$ & 964 \\
\hline Herd Creek & 534 & 0 & $(0.0)$ & 0 & & $(---)$ & -- & $(---)$ & 534 \\
\hline E. F. Salmon River & 986 & $\mathbf{0}$ & $(0.0)$ & 0 & & $(---)$ & -- & $(---)$ & 986 \\
\hline Big Creek (upper) & 757 & 0 & $(0.0)$ & 0 & & $(---)$ & -- & $(---)$ & 757 \\
\hline s. F. Salmon River & 1,574 & 1 & $(0.1)$ & 369 & 3 & $(0.8)$ & 0 & $(0.0)$ & 1,571 \\
\hline Big Creek (lower) & 727 & 0 & $(0.0)$ & 0 & - - & $(---)$ & -- & $(---)$ & 727 \\
\hline Rush Creek & 15 & 0 & $(0.0)$ & 0 & - - & $(---)$ & -- & $(---)$ & 15 \\
\hline ii. F. Chamberlain Creek & 917 & 0 & $(0.0)$ & 0 & -- & $(---)$ & -- & $(---)$ & 917 \\
\hline Chamberlain Creek & 241 & 0 & $(0.0)$ & 0 & & $(---)$ & -- & $(---)$ & 241 \\
\hline Secesh River & 1,551 & 0 & $(0.0)$ & 0 & -- & $(---)$ & -- & $(---)$ & 1,551 \\
\hline Lake Creek & 406 & 1 & (0.3) & 0 & & $(---)$ & -- & $(---)$ & 405 \\
\hline Total & 18,495 & 22 & $(0.1)$ & 2508 & 12 & $(0.5)$ & 0 & $(0.0)$ & 18,462 \\
\hline
\end{tabular}


Appendix Table 4A. Detections of PIT-tagged smolts by date at three Snake River dams for wild chinook salmon from Bear Valley Creek, 1995. Numbers in parentheses are first detections at the dams that have been adjusted for spill.

Tagging site: Bear Valley Creek

Release date: 27 - 29 July 1994

Release site: Bear Valley Creek

Number released: 1,455

Release river kilometer(s) above Lower Granite Dam: 628 - 633

\begin{tabular}{|c|c|c|c|c|c|c|}
\hline \multirow[b]{2}{*}{$\begin{array}{c}\text { Detection } \\
\text { date }\end{array}$} & \multirow{3}{*}{$\begin{array}{c}\text { Lower Granite } \\
\text { First } \\
\text { detection }\end{array}$} & \multicolumn{2}{|c|}{ Little Goose } & \multicolumn{3}{|c|}{ Lower Monumental } \\
\hline & & $\begin{array}{c}\text { First } \\
\text { detection }\end{array}$ & $\begin{array}{l}\text { Previous } \\
\text { detections } \\
\text { at } 1 \text { dam }\end{array}$ & $\begin{array}{l}\text { First } \\
\text { detection }\end{array}$ & $\begin{array}{l}\text { Previous } \\
\text { detections } \\
\text { at } 1 \text { dam }\end{array}$ & $\begin{array}{l}\text { Previous } \\
\text { detections } \\
\text { at } 2 \text { dams }\end{array}$ \\
\hline 13 Apr & & & & & & \\
\hline 16 Apr & 1 & & & & & \\
\hline 18 Apr & 1 & & & & & \\
\hline 19 Apr & 1 & & & & & \\
\hline 20 Apr & 1 & & & & & \\
\hline 21 Apr & 1 & & & & & \\
\hline 24 Apr & & & & 1 & & \\
\hline 25 Apr & 1 & & & & & \\
\hline 27 Apr & 1 & & & & & \\
\hline 28 Apr & 2 & & & & & \\
\hline 30 Apr & 1 & & & & & \\
\hline 01 May & 2 & & & & & \\
\hline 02 May & 1 & & & & & \\
\hline 03 May & 2 & & & & & \\
\hline 04 May & 1 & & & & & \\
\hline
\end{tabular}


Appendix Table 4A. Continued.

\begin{tabular}{|c|c|c|c|c|c|c|c|c|}
\hline \multirow{2}{*}{\multicolumn{2}{|c|}{$\begin{array}{l}\text { Detection } \\
\text { date }\end{array}$}} & \multirow{2}{*}{\multicolumn{2}{|c|}{$\begin{array}{c}\text { Lower Granite } \\
\text { First } \\
\text { detection }\end{array}$}} & \multicolumn{2}{|c|}{ Little Goose } & \multicolumn{3}{|c|}{ Lower Monumental } \\
\hline & & & & $\begin{array}{c}\text { First } \\
\text { detection }\end{array}$ & $\begin{array}{c}\text { Previous } \\
\text { detections } \\
\text { at } \mathbf{1} \text { dam }\end{array}$ & $\begin{array}{l}\text { First } \\
\text { detection }\end{array}$ & $\begin{array}{l}\text { Previous } \\
\text { detections } \\
\text { at } \mathbf{1} \text { dam }\end{array}$ & \multirow[t]{2}{*}{$\begin{array}{l}\text { Previous } \\
\text { detections } \\
\text { at } 2 \text { dams }\end{array}$} \\
\hline 05 & May & 1 & & 1 & & & 1 & \\
\hline 06 & May & & & 1 & & & & \\
\hline 07 & May & 1 & & & & 1 & & \\
\hline 08 & May & & & & & & & \\
\hline 09 & May & 41 & & & & & & \\
\hline 10 & May & 2 & & & & & & \\
\hline 11 & May & 21 & 3) & & & & & \\
\hline 12 & May & 1 & & & & & & 1 \\
\hline 13 & May & 2 & & $1 \quad(2)$ & & & 1 & 1 \\
\hline 14 & May & 21 & 3) & & & 2 & & \\
\hline 15 & May & 31 & 4) & & & & & \\
\hline 16 & May & & & & & & 2 & \\
\hline 18 & May & 21 & 3) & & & & & \\
\hline 19 & May & & & & & & & \\
\hline 20 & May & 21 & 3) & & & & & \\
\hline 21 & May & 21 & 3) & & & 1 & & \\
\hline 22 & May & & & & & & & 1 \\
\hline 23 & May & & & & & 1 & 1 & \\
\hline 24 & May & 21 & 3) & & & & & \\
\hline
\end{tabular}


Appendix Table 4A. Continued.

\begin{tabular}{|c|c|c|c|c|c|c|}
\hline \multirow{2}{*}{$\begin{array}{l}\text { Detection } \\
\text { date }\end{array}$} & \multirow{2}{*}{$\begin{array}{c}\text { Lower Granite } \\
\text { First } \\
\text { detection }\end{array}$} & \multicolumn{2}{|c|}{ Little Goose } & \multicolumn{3}{|c|}{ Lower Monumental } \\
\hline & & $\begin{array}{c}\text { First } \\
\text { detection }\end{array}$ & $\begin{array}{l}\text { Previous } \\
\text { detections } \\
\text { at } 1 \text { dam }\end{array}$ & $\begin{array}{l}\text { First } \\
\text { detection }\end{array}$ & $\begin{array}{l}\text { Previous } \\
\text { detections } \\
\text { at } 1 \text { dam }\end{array}$ & $\begin{array}{l}\text { Previous } \\
\text { detections } \\
\text { at } 2 \text { dams }\end{array}$ \\
\hline 25 May & 1 & 1 & & 1 & & \\
\hline 26 May & 1 & & 3 & & & 1 \\
\hline 27 May & 3 & 2 & & & & \\
\hline 28 May & 1 & & & & & \\
\hline 29 May & 4 & & 1 & & & \\
\hline 30 May & & & & & & \\
\hline 31 May & 2 & & & & & \\
\hline 01 Jun & & & & & & \\
\hline 02 Jun & 1 & & & & & \\
\hline 03 Jun & 2 & & & & & \\
\hline 04 Jun & 1 & & & & & \\
\hline 05 Jun & 1 & 2 & & & & \\
\hline 06 Jun & 1 & & & & & \\
\hline $07 J$ un & 1 & & & & & \\
\hline 08 Jun & 1 & & 2 & & & \\
\hline 09 Jun & & & 1 & 1 & & \\
\hline 10 Jun & $1 \quad 2$ & & 1 & & & \\
\hline 11 Jun & & & 1 & 1 & 1 & \\
\hline 12 Jun & 1 & & 1 & & & \\
\hline
\end{tabular}


Appendix Table 4A. Continued.

\begin{tabular}{|c|c|c|c|c|c|c|}
\hline \multirow{2}{*}{$\begin{array}{l}\text { Detection } \\
\text { date }\end{array}$} & \multirow{2}{*}{$\begin{array}{c}\text { Lower Granite } \\
\text { First } \\
\text { detection }\end{array}$} & \multicolumn{2}{|c|}{ Little Goose } & \multicolumn{3}{|c|}{ Lower Monumental } \\
\hline & & $\begin{array}{c}\text { First } \\
\text { detection }\end{array}$ & $\begin{array}{l}\text { Previous } \\
\text { detections } \\
\text { at } 1 \text { dam }\end{array}$ & $\begin{array}{l}\text { First } \\
\text { detection }\end{array}$ & $\begin{array}{l}\text { Previous } \\
\text { detections } \\
\text { at } 1 \text { dam }\end{array}$ & $\begin{array}{l}\text { Previous } \\
\text { detections } \\
\text { at } 2 \text { dams }\end{array}$ \\
\hline 13 Jun & & & 1 & & & \\
\hline 14 Jun & & 1 & & & & 2 \\
\hline 15 Jun & & & & & & 1 \\
\hline 16 Jun & & & 1 & & & \\
\hline 17 Jun & & & & & & 1 \\
\hline 18 Jun & & & & 1 & & 1 \\
\hline 20 Jun & 1 & & & & & \\
\hline 24 Jun & 1 & & & & & \\
\hline 27 Jun & 1 & & & & & \\
\hline 28 Jun & & 1 & & & & \\
\hline 30 Jun & 1 & & & & & \\
\hline $01 \mathrm{Jul}$ & & 1 & & & & \\
\hline $02 \mathrm{Jul}$ & & & & & & \\
\hline $03 \mathrm{Jul}$ & & & & & & \\
\hline $05 \mathrm{Jul}$ & & & & & & 1 \\
\hline $06 \mathrm{Jul}$ & 1 & & & & & \\
\hline $08 \mathrm{Jul}$ & 1 & & & & & \\
\hline $09 \mathrm{Jul}$ & & & & & & \\
\hline $11 \mathrm{Jul}$ & & & & & 1 & \\
\hline
\end{tabular}


Appendix Table 4.4. Continued.

\begin{tabular}{|c|c|c|c|c|c|c|}
\hline \multirow[b]{2}{*}{$\begin{array}{c}\text { Detection } \\
\text { date }\end{array}$} & \multirow{2}{*}{$\begin{array}{c}\text { Lower Granite } \\
\text { First } \\
\text { detection }\end{array}$} & \multicolumn{2}{|c|}{ Little Goose } & \multicolumn{3}{|c|}{ Lower Monumental } \\
\hline & & $\begin{array}{c}\text { First } \\
\text { detection }\end{array}$ & $\begin{array}{l}\text { Previous } \\
\text { detections } \\
\text { at } 1 \text { dam }\end{array}$ & $\begin{array}{l}\text { First } \\
\text { detection }\end{array}$ & $\begin{array}{l}\text { Previous } \\
\text { detections } \\
\text { at } 1 \text { dam }\end{array}$ & $\begin{array}{l}\text { Previous } \\
\text { detections } \\
\text { at } 2 \text { dams }\end{array}$ \\
\hline $13 \mathrm{Jul}$ & 1 & & & & & \\
\hline $14 \mathrm{JuI}$ & & & 1 & & & \\
\hline $17 \mathrm{Jul}$ & & & 2 & & & 1 \\
\hline 19 Jul & & & 1 & & & \\
\hline $20 \mathrm{Jul}$ & 1 & & & & & \\
\hline $21 \mathrm{Jul}$ & & & & & & 1 \\
\hline 05 Aug & & & & & & 1 \\
\hline 06 Aug & & & & 1 & & \\
\hline .27 Aug & & & & 1 & & \\
\hline Totals & $73 \quad(82)$ & $(20)$ & 28 & 14 & 21 & 17 \\
\hline
\end{tabular}


Appendix Table 4B. Detections of PIT-tagged smolts by date at three Columbia River dams for wild chinook salmon from Bear Valley Creek. 1995. Numbers in parentheses are first detections at the dams that have been adjusted for spill.

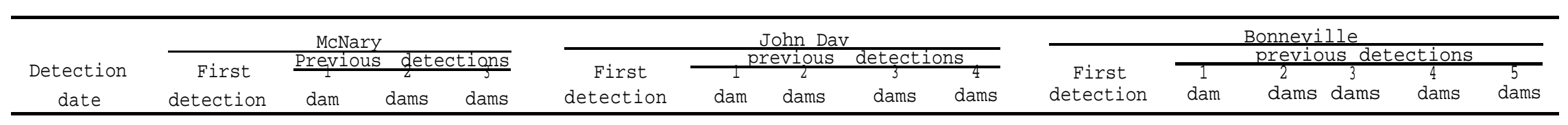

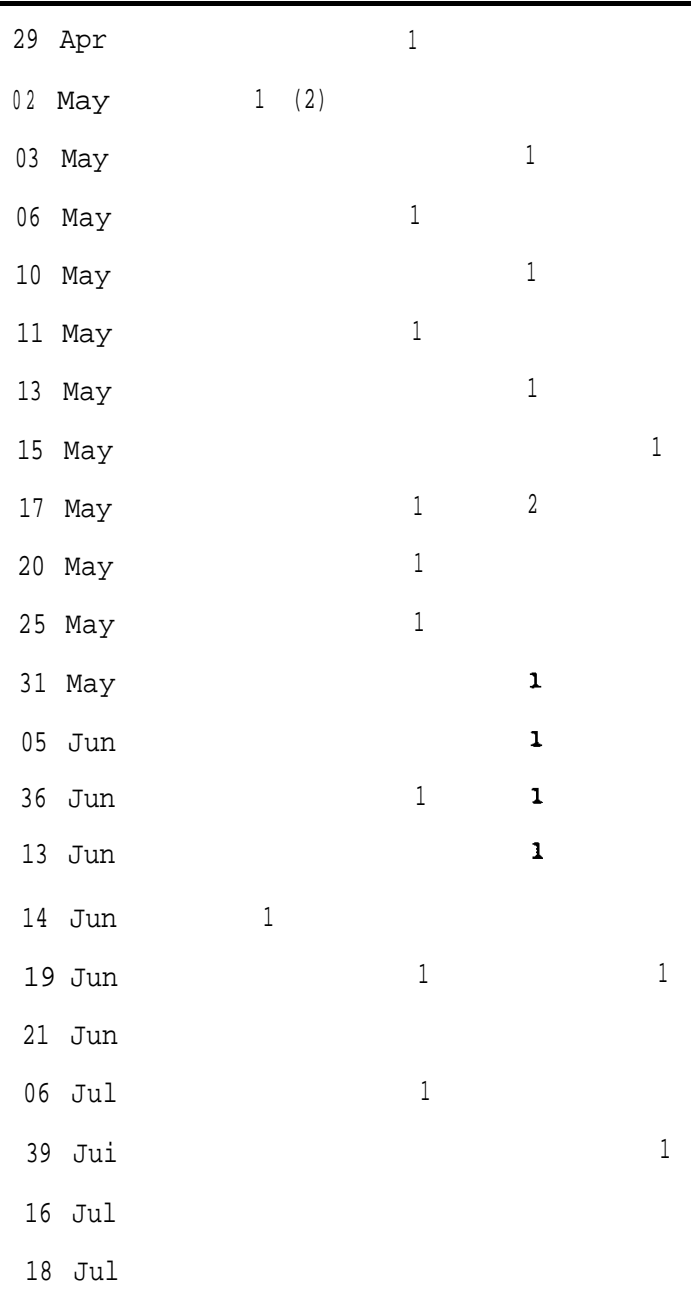


Appendix Table 4B. Continued.

\begin{tabular}{|c|c|c|c|c|c|c|c|c|c|c|c|c|c|c|c|}
\hline \multirow[b]{2}{*}{$\begin{array}{l}\text { Detection. } \\
\text { date }\end{array}$} & \multicolumn{4}{|c|}{ McNary } & \multicolumn{5}{|c|}{ John Day } & \multicolumn{6}{|c|}{ Bonneviile } \\
\hline & $\begin{array}{c}\text { Birst } \\
\text { detectior. }\end{array}$ & $\begin{array}{c}1 \\
\text { dam }\end{array}$ & $\begin{array}{c}2 \\
\text { dams }\end{array}$ & $\begin{array}{c}3 \\
\text { dams }\end{array}$ & $\begin{array}{c}\text { First } \\
\text { detection }\end{array}$ & $\begin{array}{c}1 \\
\text { dam }\end{array}$ & $\begin{array}{c}\frac{2}{2} \\
\text { dams }\end{array}$ & $\begin{array}{c}3 \\
\text { dams }\end{array}$ & $\begin{array}{c}\frac{15}{4 a m s} \\
\text { dats }\end{array}$ & $\begin{array}{c}\text { First } \\
\text { detection }\end{array}$ & $\begin{array}{c}1 \\
\text { dam }\end{array}$ & $\begin{array}{c}2 \\
\text { dams }\end{array}$ & dams & dams & $\begin{array}{c}5 \\
\text { dams }\end{array}$ \\
\hline 06 sep & & 1 & & & & & & & & & & & & & \\
\hline Totals & $2(3)$ & 10 & 13 & 3 & & & & 1 & & & & & & & \\
\hline
\end{tabular}


Appendix Table 5A Detections of PIT-tagged smolts by date at three Snake River dams for wild chinook salmon from Elk Creek, 1995. Numbers in parentheses are first detections at the dams that have been adjusted for spill.

Tagging site: Elk Creek

Release date: 29 July - 01 Aug 1994

Release site: Elk Creek

Number released: 1,512

Release river kilometer(s) above Lower Granite Dam: 634 - 637

\begin{tabular}{|c|c|c|c|c|c|c|}
\hline \multirow{2}{*}{$\begin{array}{l}\text { Detection } \\
\text { date }\end{array}$} & \multirow{3}{*}{$\begin{array}{c}\text { Lower Granite } \\
\begin{array}{c}\text { First } \\
\text { detection }\end{array} \\
1\end{array}$} & \multirow{2}{*}{\multicolumn{2}{|c|}{$\begin{array}{cc}\text { Little } & \text { Goose } \\
\text { First } & \text { detectious } \\
\text { detection } & \text { at } 1 \text { dam }\end{array}$}} & \multicolumn{3}{|c|}{ Lower Monumental } \\
\hline & & & & $\begin{array}{l}\text { First } \\
\text { detection }\end{array}$ & $\begin{array}{l}\text { Previous } \\
\text { detections } \\
\text { at } 1 \text { dam }\end{array}$ & $\begin{array}{l}\text { Previous } \\
\text { detections } \\
\text { at } 2 \text { dams }\end{array}$ \\
\hline 10 Apr & & & & & & \\
\hline 11 Apr & 1 & & & & & \\
\hline 12 Apr & 1 & & & & & \\
\hline 13 Apr & 1 & & & & & \\
\hline 15 Apr & 1 & & & & & \\
\hline 17 Apr & 2 & & & & & \\
\hline 18 Apr & 2 & & & & & \\
\hline 20 Apr & 1 & & & & & \\
\hline 21 Apr & 3 & & & & 1 & \\
\hline 22 Apr & & & & 1 & & \\
\hline 23 Apr & 1 & & & & & \\
\hline 26 Apr & 2 & & & & 1 & \\
\hline 27 Apr & 1 & & & & & \\
\hline 28 Apr & 3 & & & & & \\
\hline 29 Apr & 3 & & & & 1 & \\
\hline
\end{tabular}


Appendix Table 5A. Continued.

\begin{tabular}{|c|c|c|c|c|c|c|c|c|c|c|}
\hline \multirow{2}{*}{\multicolumn{2}{|c|}{$\begin{array}{l}\text { Detection } \\
\text { date }\end{array}$}} & \multirow{2}{*}{\multicolumn{2}{|c|}{$\begin{array}{c}\text { Lower Granite } \\
\text { First } \\
\text { detection }\end{array}$}} & \multicolumn{3}{|c|}{ Little Goose } & \multicolumn{4}{|c|}{ Lower Monumental } \\
\hline & & & & \multicolumn{2}{|c|}{$\begin{array}{c}\text { First } \\
\text { detection }\end{array}$} & $\begin{array}{l}\text { Previous } \\
\text { detections } \\
\text { at } 1 \text { dam }\end{array}$ & \multicolumn{2}{|c|}{$\begin{array}{l}\text { First } \\
\text { detection }\end{array}$} & \multirow[t]{2}{*}{$\begin{array}{l}\text { Previous } \\
\text { detections } \\
\text { at } 1 \text { dam }\end{array}$} & \multirow[t]{2}{*}{$\begin{array}{l}\text { Previous } \\
\text { detections } \\
\text { at } 2 \text { dams }\end{array}$} \\
\hline 30 & Apr & 1 & & & & & & & & \\
\hline 01 & May & 1 & & & & 1 & 1 & & & \\
\hline 02 & May & & & 1 & & & & & & \\
\hline 03 & May & 2 & & 1 & & 1 & 1 & & & \\
\hline 04 & May & 1 & & & & & & & & \\
\hline 05 & May & 1 & & & & 1 & 1 & & & \\
\hline 06 & May & & & 1 & & 1 & 1 & & & \\
\hline 07 & May & 1 & & & & & & & & 1 \\
\hline 08 & May & & & 1 & & & 2 & 3 & & \\
\hline 09 & May & 6 & 7 & 1 & & & & & & \\
\hline 10 & May & 2 & & 1 & & & 1 & & & \\
\hline 11 & May & 1 & & 2 & 3 & & & & & \\
\hline 12 & May & & & & & & 1 & & & 1 \\
\hline 13 & May & 2 & & 1 & 2 & & & & & 1 \\
\hline 14 & May & 5 & 6 & & & & & & & 1 \\
\hline 16 & May & & & & & & 1 & & & \\
\hline 17 & May & & & 1 & & & 1 & & & \\
\hline 18 & May & & & 1 & & & & & & \\
\hline 19 & May & 2 & 3 & & & & & & & \\
\hline
\end{tabular}


Appendix Table 5A. Continued.

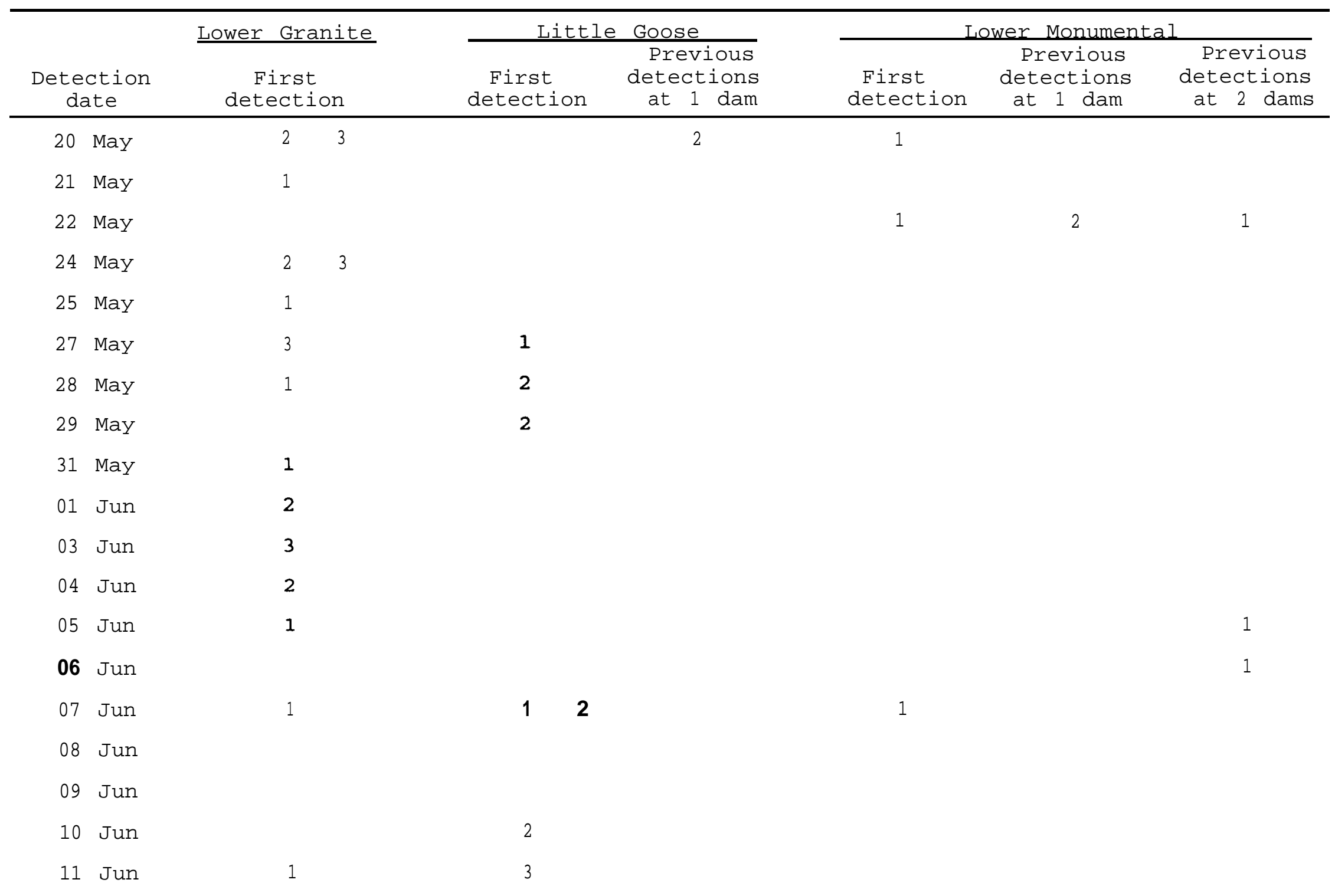


Appendix Table 5A. Continued.

\begin{tabular}{|c|c|c|c|c|c|c|}
\hline \multirow[b]{2}{*}{$\begin{array}{c}\text { Detection } \\
\text { date }\end{array}$} & \multirow{3}{*}{$\begin{array}{c}\text { Lower Granite } \\
\begin{array}{c}\text { First } \\
\text { detection }\end{array} \\
1\end{array}$} & \multicolumn{2}{|c|}{ Little Goose } & \multicolumn{3}{|c|}{ Lower Monumental } \\
\hline & & $\begin{array}{c}\text { First } \\
\text { detection }\end{array}$ & $\begin{array}{r}\text { Previous } \\
\text { detections } \\
\text { at } 1 \text { dam }\end{array}$ & $\begin{array}{l}\text { First } \\
\text { detection }\end{array}$ & $\begin{array}{l}\text { Previous } \\
\text { detections } \\
\text { at } 1 \text { dam }\end{array}$ & $\begin{array}{l}\text { Previous } \\
\text { detections } \\
\text { at } 2 \text { dams }\end{array}$ \\
\hline 12 Jun & & & & & & \\
\hline 13 Jun & & & & & 1 & \\
\hline 14 Jun & & 1 & & & & 1 \\
\hline 15 Jun & & 1 & & & & \\
\hline 16 Jun & & 1 & & & 1 & \\
\hline 19 Jun & 2 & & & & 2 & \\
\hline 22 Jun & & & & & & \\
\hline 25 Jun & & & & & & \\
\hline 26 Jun & 1 & & & & & \\
\hline 28 Jun & & & & & & \\
\hline 29 Jun & & 1 & & & & \\
\hline $01 \mathrm{Jul}$ & & & & & & \\
\hline $04 \mathrm{Jul}$ & & & & & & \\
\hline $05 \mathrm{Jul}$ & 1 & 1 & & & & \\
\hline $06 \mathrm{Jul}$ & & & & & 1 & \\
\hline $09 \mathrm{Jul}$ & 1 & & & & & \\
\hline $14 \mathrm{Jul}$ & & & & & 1 & \\
\hline
\end{tabular}


Appendix Table 5A. Continued.

\begin{tabular}{|c|c|c|c|c|c|c|}
\hline \multirow[b]{2}{*}{$\begin{array}{c}\text { Detection } \\
\text { date }\end{array}$} & \multirow{2}{*}{$\begin{array}{c}\text { Lower Granite } \\
\text { First } \\
\text { detection }\end{array}$} & \multicolumn{2}{|c|}{ Little Goose } & \multicolumn{3}{|c|}{ Lower Monumental } \\
\hline & & $\begin{array}{c}\text { First } \\
\text { detection }\end{array}$ & $\begin{array}{r}\text { Previous } \\
\text { detections } \\
\text { at } 1 \text { dam }\end{array}$ & $\begin{array}{l}\text { First } \\
\text { detection }\end{array}$ & $\begin{array}{l}\text { Previous } \\
\text { detections } \\
\text { at } 1 \text { dam }\end{array}$ & $\begin{array}{l}\text { Previous } \\
\text { detections } \\
\text { at } 2 \text { dams }\end{array}$ \\
\hline $24 \mathrm{Jul}$ & & & & & 1 & \\
\hline Totals & $(80)$ & (31) & 18 & (17) & 23 & 8 \\
\hline
\end{tabular}


Appendix Table 5B. Detections of PIT-tagged smolts by date at three Columbia River dams for wild chinook salmon from Elk Creek, 1995. Numbers in parentheses are first detections at the dams that have been adjusted for spill.

\begin{tabular}{|c|c|c|c|c|c|c|c|c|c|c|c|c|c|c|c|}
\hline \multirow{3}{*}{$\begin{array}{l}\text { Dezecsicn } \\
\text { date }\end{array}$} & \multicolumn{4}{|c|}{ McNary } & \multicolumn{5}{|c|}{ John Day } & \multicolumn{6}{|c|}{ Bonneville } \\
\hline & \multirow{2}{*}{$\begin{array}{c}\text { Firs: } \\
\text { detect-on }\end{array}$} & \multirow{2}{*}{\multicolumn{3}{|c|}{$\begin{array}{ccc}\text { Previous } & \text { detections } \\
1 & 2 & 3 \\
\text { dam } & \text { dams } & \text { dams }\end{array}$}} & \multirow{2}{*}{$\begin{array}{c}\text { First } \\
\text { detection }\end{array}$} & \multicolumn{4}{|c|}{ Previous detections } & \multirow{2}{*}{$\begin{array}{c}\text { First } \\
\text { detection }\end{array}$} & \multirow{2}{*}{$\frac{1}{d a c}$} & \multicolumn{4}{|c|}{ Previous detections } \\
\hline & & & & & & $\begin{array}{c}1 \\
\text { dam }\end{array}$ & $\begin{array}{c}2 \\
\text { dams }\end{array}$ & $\begin{array}{c}3 \\
\text { dams }\end{array}$ & $\frac{4}{\text { dans }}$ & & & $\begin{array}{c}2 \\
\text { dams }\end{array}$ & $\begin{array}{c}3 \\
\text { dams }\end{array}$ & $\begin{array}{c}4 \\
\text { dams }\end{array}$ & $\begin{array}{c}\frac{5}{d a m s} \\
\text { das }\end{array}$ \\
\hline $23 \mathrm{Apr}$ & & 1 & & & & & & & & & & & & & \\
\hline 01 May & & 1 & 1 & & & & & & & & & & & & \\
\hline C3 May & & 1 & & & & & & & & & & & & & \\
\hline 04 May & & i & & & & & & & & & & & & & \\
\hline 36 May & & 1 & & & & & & & & & & & & & \\
\hline 37 May & & 1 & & & & & & & & & & & & & \\
\hline ay & & 1 & 1 & & & & & & & & & & & & \\
\hline 11 Kay & & 1 & & & & & & & & & & & & & \\
\hline 12 Kay & & 1 & & & & & & & & & & & & & \\
\hline 13 нау & 1 (2) & & & & & & & & & & & & & & \\
\hline 14 !-lay & & 1 & & & & & & & & & & & & & \\
\hline 15 May & $\begin{array}{l}-\quad(2) \\
\end{array}$ & & & & & & & & & & & & & & \\
\hline 15 May & & & 1 & & & & & & & & & & & & \\
\hline 18 May & 1,2 & & & & & & & & & & & & & & \\
\hline 20 May & & & & & & & & & & & & & & & \\
\hline 21 May & & & 1 & & & & & & & & & & & & \\
\hline $32 \mathrm{Jun}$ & & & 1 & & & & & & & & & & & & \\
\hline 33 Jun & & & 1 & & & & & & & & & & & & \\
\hline $34 \mathrm{Jun}$ & & 1 & & & & & & & & & & & & & \\
\hline 06 Jun & & 1 & & & & & & & & & & & & & \\
\hline 13 Jun & & & & & & & & & & & & & & & \\
\hline 18 Jun & & & & : & & & & & & & & & & & \\
\hline
\end{tabular}


Appendix Table 5B. Continued.

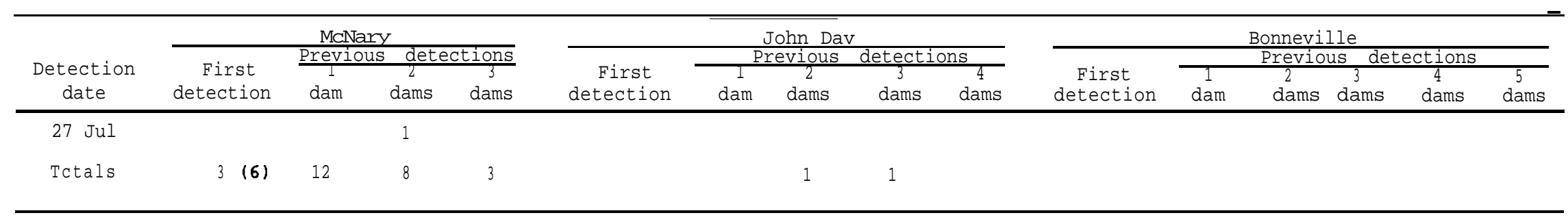


Appendix Table 6A. Detections of PIT-tagged smolts by date at three Snake River dams for wild chinook salmon from Sulphur Creek, 1995. Numbers in parentheses are first detections at the dams that have been adjusted for spill.

Tagging site: Sulphur Creek

Release site: Sulphur Creek

Release date: 31 July - 01 Aug 1994

Release river kilometer(s) above Lower Granite Dam: 604 - 606

$$
\text { Number released: } 728
$$

\begin{tabular}{|c|c|c|c|c|c|c|}
\hline \multirow{2}{*}{$\begin{array}{l}\text { Detection } \\
\text { date }\end{array}$} & \multirow{2}{*}{$\begin{array}{c}\text { Lower Granite } \\
\text { First } \\
\text { detection }\end{array}$} & \multicolumn{2}{|c|}{ Little Goose } & \multicolumn{3}{|c|}{ Lower Monumental } \\
\hline & & $\begin{array}{c}\text { First } \\
\text { detection }\end{array}$ & $\begin{array}{l}\text { Previous } \\
\text { detections } \\
\text { at } 1 \text { dam }\end{array}$ & $\begin{array}{l}\text { First } \\
\text { detection }\end{array}$ & $\begin{array}{l}\text { Previous } \\
\text { detections } \\
\text { at } 1 \text { dam }\end{array}$ & $\begin{array}{l}\text { Previous } \\
\text { detections } \\
\text { at } 2 \text { dams }\end{array}$ \\
\hline 11 Apr & 2 & & & & & \\
\hline 14 Apr & 1 & & & & & \\
\hline 16 Apr & & & 1 & & & \\
\hline 18 Apr & 1 & & & & & \\
\hline 19 Apr & & & & & & \\
\hline 21 Apr & & & & & & \\
\hline 25 Apr & 1 & & & & & \\
\hline 26 Apr & & & & & & \\
\hline 28 Apr & 1 & & & & & \\
\hline 02 May & 1 & & & & & \\
\hline 03 May & & & & & 1 & \\
\hline 04 May & & & & & & \\
\hline 05 May & & & & & 1 & \\
\hline 06 May & 3 & & & & & \\
\hline 07 May & & & & & & \\
\hline
\end{tabular}


Appendix Table 6A. Continued.

\begin{tabular}{|c|c|c|c|c|c|c|c|}
\hline \multirow{2}{*}{\multicolumn{2}{|c|}{$\begin{array}{l}\text { Detection } \\
\text { date }\end{array}$}} & \multirow{2}{*}{$\begin{array}{c}\text { Lower Granite } \\
\text { First } \\
\text { detection }\end{array}$} & \multicolumn{2}{|c|}{ Little Goose } & \multicolumn{3}{|c|}{ Lower Monumental } \\
\hline & & & $\begin{array}{c}\text { First } \\
\text { detection }\end{array}$ & $\begin{array}{r}\text { Previous } \\
\text { detections } \\
\text { at } \mathbf{1} \text { dam }\end{array}$ & $\begin{array}{l}\text { First } \\
\text { detection }\end{array}$ & $\begin{array}{l}\text { Previous } \\
\text { detections } \\
\text { at } \mathbf{1} \text { dam }\end{array}$ & $\begin{array}{l}\text { Previous } \\
\text { detections } \\
\text { at } 2 \text { dams }\end{array}$ \\
\hline 08 & May & 1 & 2 (3) & & & 1 & \\
\hline 09 & May & $5 \quad 6)$ & & & $2 \quad 3)$ & & \\
\hline 10 & May & 2 & 1 & & & 1 & \\
\hline 11 & May & & \begin{tabular}{l|l}
5 & $(7)$
\end{tabular} & & & & \\
\hline 12 & May & 1 & & & & & \\
\hline 13 & May & & $2 \quad(3)$ & & 1 & 4 & \\
\hline 14 & May & 1 & $1 \mid 2$ & & & & \\
\hline 15 & May & 1 & & & 1 & & \\
\hline 16 & May & 1 & $2 \quad(3)$ & & & 1 & \\
\hline 1 & May & 1 & 1 & & $4(5)$ & 1 & \\
\hline 1 & May & 1 & & & & & \\
\hline 19 & May & & & & & 1 & \\
\hline 20 & May & & & 1 & 1 & & \\
\hline 21 & May & 1 & & & & & \\
\hline 22 & May & $2 \quad 3)$ & & & 1 & & \\
\hline 23 & May & $2(3)$ & & & $2(3)$ & & \\
\hline 24 & May & 1 & \begin{tabular}{l|l}
2 & 1 \\
3
\end{tabular} & & & & \\
\hline 25 & May & 2 & & 2 & 1 & 1 & \\
\hline 26 & May & 1 & 2 & 1 & & 1 & \\
\hline
\end{tabular}


Appendix Table 6A. Continued.

\begin{tabular}{|c|c|c|c|c|c|c|}
\hline \multirow{2}{*}{$\begin{array}{c}\text { Detection } \\
\text { date }\end{array}$} & \multirow{3}{*}{ 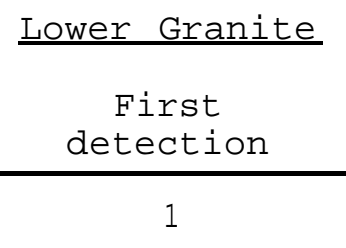 } & \multicolumn{2}{|c|}{ Little Goose } & \multicolumn{3}{|c|}{ Lower Monumental } \\
\hline & & \multirow{2}{*}{$\frac{\begin{array}{c}\text { First } \\
\text { detection }\end{array}}{1}$} & \multirow{2}{*}{$\begin{array}{l}\text { Previous } \\
\text { detections } \\
\text { at } 1 \text { dam } \\
2\end{array}$} & \multirow[t]{2}{*}{$\begin{array}{l}\text { First } \\
\text { detection }\end{array}$} & \multirow{2}{*}{$\begin{array}{c}\text { Previous } \\
\text { detections } \\
\text { at } 1 \text { dam } \\
1\end{array}$} & \multirow[t]{2}{*}{$\begin{array}{l}\text { Previous } \\
\text { detections } \\
\text { at } 2 \text { dams }\end{array}$} \\
\hline 27 May & & & & & & \\
\hline 28 May & 5 & & 1 & & 1 & 1 \\
\hline 29 May & 2 & 1 & & & 1 & 1 \\
\hline 30 May & & & 2 & & & \\
\hline 31 May & & & & & 1 & \\
\hline 01 Jun & 2 & & 1 & & & 2 \\
\hline 03 Jun & 1 & 1 & & & & \\
\hline 04 Jun & 1 & 1 & 1 & & 1 & \\
\hline 05 Jun & 2 & & & 1 & 1 & \\
\hline 06 Jun & $3(4)$ & & & & & \\
\hline 07 Jun & & & 2 & & & \\
\hline 08 Jun & & & 2 & & & \\
\hline 09 Jun & 1 & & & & 1 & \\
\hline 10 Jun & & & & & 1 & 2 \\
\hline 11 Jun & & & 1 & & & \\
\hline 12 Jun & & & 1 & & 3 & \\
\hline 13 Jun & & & & 1 & & \\
\hline 14 Jun & 1 & & & & & \\
\hline 15 Jun & 1 & & & & & \\
\hline
\end{tabular}


Appendix Table 6A. Continued.

\begin{tabular}{|c|c|c|c|c|c|c|}
\hline \multirow[b]{2}{*}{$\begin{array}{c}\text { Detection } \\
\text { date }\end{array}$} & \multirow{2}{*}{$\begin{array}{c}\text { Lower Granite } \\
\text { First } \\
\text { detection }\end{array}$} & \multicolumn{2}{|c|}{ Little Goose } & \multicolumn{3}{|c|}{ Lower Monumental } \\
\hline & & $\begin{array}{c}\text { First } \\
\text { detection }\end{array}$ & $\begin{array}{r}\text { Previous } \\
\text { detections } \\
\text { at } 1 \text { dam }\end{array}$ & $\begin{array}{l}\text { First } \\
\text { detection }\end{array}$ & $\begin{array}{c}\text { Previous } \\
\text { detections } \\
\text { at } 1 \text { dam }\end{array}$ & $\begin{array}{l}\text { Previous } \\
\text { detections } \\
\text { at } 2 \text { dams }\end{array}$ \\
\hline 17 Jun & & & & & 2 & \\
\hline 21 Jun & 1 & 1 & & & & \\
\hline 23 Jun & 1 & & 1 & & & \\
\hline 30 Jun & & & 1 & & & \\
\hline $01 \mathrm{Jul}$ & 1 & & 1 & & & \\
\hline $02 \mathrm{Jul}$ & 1 & & & & & \\
\hline $03 \mathrm{Jul}$ & & & & & & 1 \\
\hline $05 \mathrm{Jul}$ & & & 1 & & & \\
\hline $07 \mathrm{Jul}$ & & & & & & 1 \\
\hline $09 \mathrm{Jul}$ & & & 1 & & & \\
\hline $14 \mathrm{Jul}$ & & & & & & 1 \\
\hline Totals & $56 \quad(61)$ & $34 \quad(41)$ & 27 & $18 \quad(21)$ & 26 & 12 \\
\hline
\end{tabular}

\section{๙ิ}


Appendix Table 6B. Detections of PIT-tagged smolts by date at three Columbia River dams for wild chinook salmon from Sulphur Creek, 1995. Numbers in parentheses are first detections at the darns that have been adjusted for spill.

\begin{tabular}{|c|c|c|c|c|c|c|c|c|c|c|c|c|c|c|}
\hline \multirow[b]{3}{*}{$\begin{array}{l}\text { Detecticn } \\
\text { date }\end{array}$} & \multicolumn{3}{|c|}{ McNarv } & \multicolumn{5}{|c|}{ John Day } & \multicolumn{6}{|c|}{ Bonneville } \\
\hline & & Previous det & tions & & & vious & etect & & & & revio & s det & ctions & \\
\hline & $\begin{array}{c}\text { Firs: } \\
\text { detection }\end{array}$ & $\begin{array}{cc}1 & 2 \\
\text { dam } & \text { dams }\end{array}$ & $\begin{array}{c}3 \\
\text { dams }\end{array}$ & $\begin{array}{c}\text { First } \\
\text { detection }\end{array}$ & $\begin{array}{c}1 \\
\text { dam }\end{array}$ & $\begin{array}{c}2 \\
\text { dams }\end{array}$ & $\begin{array}{c}3 \\
\text { dams }\end{array}$ & $\frac{4}{d a m s}$ & $\begin{array}{c}\text { First } \\
\text { detection }\end{array}$ & $\begin{array}{c}1 \\
\text { dam }\end{array}$ & dams & dams & $\begin{array}{c}4 \\
\text { dams }\end{array}$ & $\begin{array}{l}5 \\
\text { dams }\end{array}$ \\
\hline
\end{tabular}

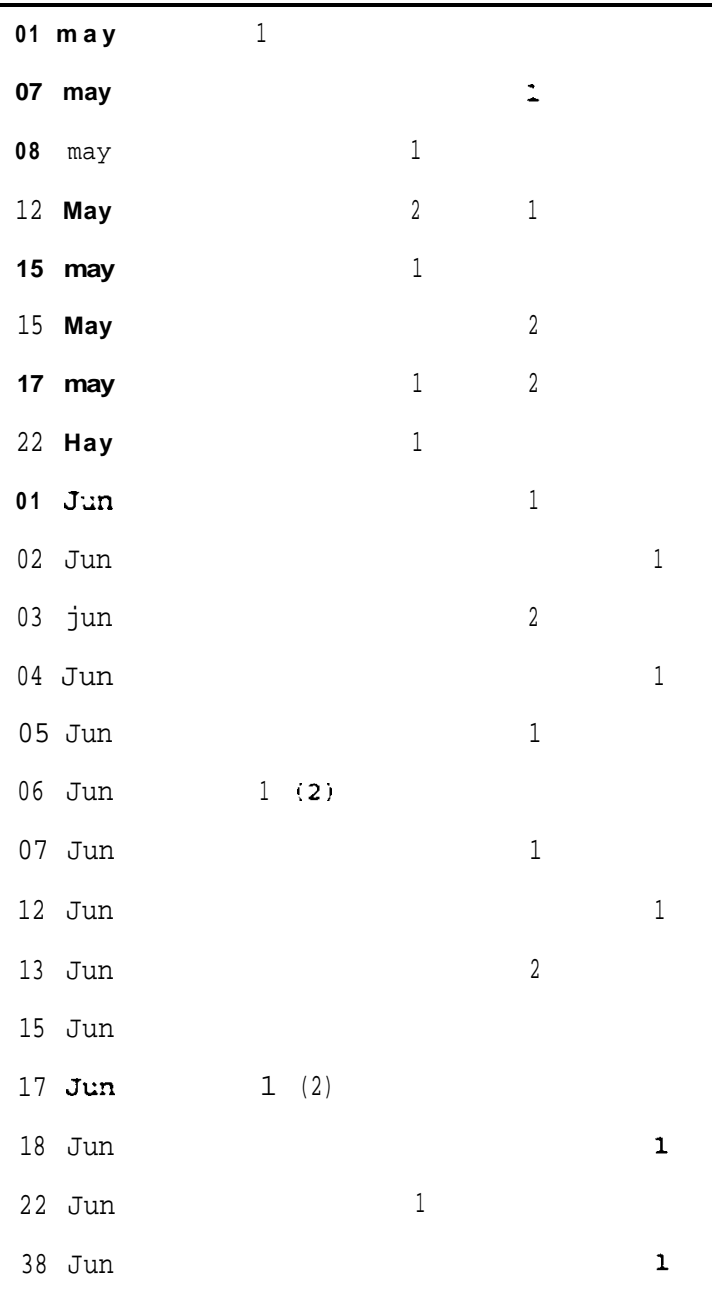


Appendix Table 6B. Continued.

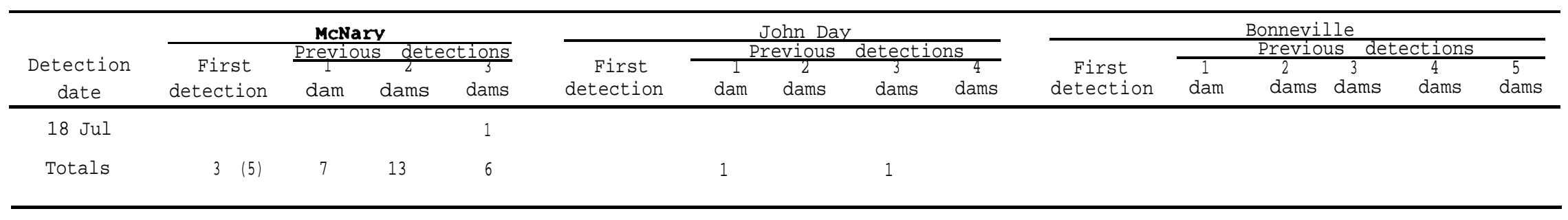


Appendix Table 7A. Detections of PIT-tagged smolts by date at three Snake River dams for wild chinook salmon from Marsh Creek, 1995. Numbers in parentheses are first detections at the darns that have been adjusted for spill.

Tagging site: Marsh Creek

Release site: Marsh Creek

Release river kilometer(s) above Lower Granite Dam: 630 - 633
Release date: 02 - 03 Aug 1994

Number released: 1,575

\begin{tabular}{|c|c|c|c|c|c|c|}
\hline \multirow{2}{*}{$\begin{array}{c}\text { Detection } \\
\text { date }\end{array}$} & \multirow{3}{*}{$\begin{array}{c}\text { Lower Granite } \\
\begin{array}{c}\text { First } \\
\text { detection }\end{array} \\
2\end{array}$} & \multicolumn{2}{|c|}{ Little Goose } & \multicolumn{3}{|c|}{ Lower Monumental } \\
\hline & & $\begin{array}{c}\text { First } \\
\text { detection }\end{array}$ & $\begin{array}{r}\text { Previous } \\
\text { detections } \\
\text { at } 1 \text { dam }\end{array}$ & $\begin{array}{l}\text { First } \\
\text { detection }\end{array}$ & $\begin{array}{l}\text { Previous } \\
\text { detections } \\
\text { at } 1 \text { dam }\end{array}$ & $\begin{array}{l}\text { Previous } \\
\text { detections } \\
\text { at } 2 \text { dams }\end{array}$ \\
\hline 11 Apr & & & & & & \\
\hline 12 Apr & 4 & & & & & \\
\hline 13 Apr & 2 & & & & & \\
\hline 14 Apr & 2 & & & & & \\
\hline 15 Apr & & & & & & \\
\hline 16 Apr & 1 & & & & & \\
\hline 17 Apr & 1 & & & & & \\
\hline 18 Apr & 2 & & & & & \\
\hline 19 Apr & 1 & & & & & 3 \\
\hline 20 Apr & 3 & & & & & \\
\hline 21 Apr & 1 & & & & & \\
\hline 22 Apr & & 2) & & & & \\
\hline 24 Apr & 2 & & & & & 1 \\
\hline 26 Apr & 1 & & & & & \\
\hline 27 Apr & 1 & & & & & \\
\hline
\end{tabular}


Appendix Table 7A. Continued.

\begin{tabular}{|c|c|c|c|c|c|c|c|c|c|c|}
\hline \multirow{2}{*}{\multicolumn{2}{|c|}{$\begin{array}{c}\text { Detection } \\
\text { date }\end{array}$}} & & \multicolumn{3}{|c|}{ Little Goose } & \multicolumn{4}{|c|}{ Lower Monumental } \\
\hline & & & & $\begin{array}{l}\text { First } \\
\text { detect }\end{array}$ & ion & $\begin{array}{r}\text { Previous } \\
\text { detections } \\
\text { at } 1 \text { dam } \\
\end{array}$ & \multicolumn{2}{|c|}{$\begin{array}{l}\text { First } \\
\text { detection }\end{array}$} & \multirow{2}{*}{$\begin{array}{c}\text { Previous } \\
\text { detections } \\
\text { at } 1 \text { dam } \\
1\end{array}$} & \multirow[t]{2}{*}{$\begin{array}{l}\text { Previous } \\
\text { detections } \\
\text { at } 2 \text { dams }\end{array}$} \\
\hline 28 & Apr & \multicolumn{2}{|c|}{$\begin{array}{c}\text { Lower Granite } \\
\begin{array}{c}\text { First } \\
\text { detection }\end{array} \\
5\end{array}$} & 1 & & & & & & \\
\hline 29 & Apr & 2 & & 1 & & & 1 & & 1 & \\
\hline 30 & Apr & 2 & & & & & & & & \\
\hline 01 & May & 5 & & & & 2 & & & & \\
\hline 02 & May & 4 & & 1 & & & & & & \\
\hline 03 & May & 3 & & & & 1 & & & & \\
\hline 04 & May & 21 & 3) & 1 & & 2 & & & 2 & \\
\hline 05 & May & 21 & 3) & 1 & & & 2 & & & \\
\hline 06 & May & 1 & & 3 & ( 41 & 1 & 21 & 31 & 2 & 2 \\
\hline 07 & May & 1 & & & & 1 & & & 3 & \\
\hline 08 & May & & & 2 & ( 3) & 1 & & & 4 & 1 \\
\hline 09 & May & 61 & 7) & 3 & (4) & & & & & 1 \\
\hline 10 & May & 41 & 5) & & & & & & 1 & \\
\hline 1 & May & 51 & 6) & 4 & ( 6) & 4 & & & 2 & \\
\hline 12 & May & 41 & 5) & 1 & & & 1 & & 1 & 1 \\
\hline 13 & May & 3 & 4) & 1 & (2) & 1 & 21 & 3) & 3 & \\
\hline 14 & May & 4 & 5) & 1 & (2) & 2 & 2 & & 1 & \\
\hline 15 & May & 61 & 8) & 1 & & & & & 1 & \\
\hline 16 & May & & & & & 1 & 1 & & 1 & \\
\hline
\end{tabular}


Appendix Table 7A. Continued.

\begin{tabular}{|c|c|c|c|c|c|c|c|c|}
\hline \multirow{2}{*}{\multicolumn{2}{|c|}{$\begin{array}{l}\text { Detection } \\
\text { date }\end{array}$}} & \multirow{2}{*}{\multicolumn{2}{|c|}{$\begin{array}{c}\text { Lower Granite } \\
\text { First } \\
\text { detection }\end{array}$}} & \multicolumn{2}{|c|}{ Little Goose } & \multicolumn{3}{|c|}{ Lower Monumental } \\
\hline & & & & $\begin{array}{l}\text { First } \\
\text { detection }\end{array}$ & $\begin{array}{l}\text { Previous } \\
\text { detections } \\
\text { at } 1 \text { dam }\end{array}$ & \multirow{2}{*}{$\frac{\begin{array}{c}\text { First } \\
\text { detection }\end{array}}{1}$} & $\begin{array}{l}\text { Previous } \\
\text { detections } \\
\text { at } 1 \text { dam }\end{array}$ & \multirow[t]{2}{*}{$\begin{array}{l}\text { Previous } \\
\text { detections } \\
\text { at } 2 \text { dams }\end{array}$} \\
\hline 17 & May & & & 1 & 2 & & & \\
\hline 18 & May & 21 & 3) & $3 \quad 4)$ & 3 & & 4 & \\
\hline 19 & May & 1 & & 1 & & 1 & & 1 \\
\hline 20 & May & 21 & 3) & $2(3)$ & 1 & 1 & 1 & 1 \\
\hline 21 & May & 21 & 3) & & & 1 & 1 & \\
\hline 22 & May & 1 & & & & & 3 & \\
\hline 23 & May & 1 & & $2(3)$ & & & & \\
\hline 24 & May & 1 & & 1 & & & & \\
\hline 25 & May & 2 & & 1 & & & & \\
\hline 26 & May & & & & & 1 & & \\
\hline 27 & May & 3 & & & & & & \\
\hline 28 & May & & & 1 & & 1 & & 2 \\
\hline 29 & May & & & & & & & 2 \\
\hline 01 & Jun & 3 & & 1 & & & & \\
\hline 02 & Jun & & & & & & & \\
\hline 03 & Jun & & & & & & & 1 \\
\hline 05 & Jun & & & & & & & 1 \\
\hline 07 & Jun & & & & & & & \\
\hline 08 & Jun & & & & & & & 1 \\
\hline
\end{tabular}


Appendix Table 7A. Continued.

\begin{tabular}{|c|c|c|c|c|c|c|}
\hline \multirow[b]{2}{*}{$\begin{array}{c}\text { Detection } \\
\text { date }\end{array}$} & \multirow{2}{*}{$\begin{array}{c}\text { Lower Granite } \\
\text { First } \\
\text { detection }\end{array}$} & \multicolumn{2}{|c|}{ Little Goose } & \multicolumn{3}{|c|}{ Lower Monumental } \\
\hline & & $\begin{array}{c}\text { First } \\
\text { detection }\end{array}$ & $\begin{array}{l}\text { Previous } \\
\text { detections } \\
\text { at } 1 \text { dam }\end{array}$ & $\begin{array}{l}\text { First } \\
\text { detection }\end{array}$ & $\begin{array}{l}\text { Previous } \\
\text { detections } \\
\text { at } 1 \text { dam }\end{array}$ & $\begin{array}{l}\text { Previous } \\
\text { detections } \\
\text { at } 2 \text { dams }\end{array}$ \\
\hline 09 Jun & & & & 1 & & \\
\hline 11 Jun & & & 1 & 1 & & 1 \\
\hline 14 Jun & & & & & & 1 \\
\hline 19 Jun & 1 & & & & & \\
\hline 23 Jun & & & 1 & & & \\
\hline 28 Jun & & & & 1 & & \\
\hline $08 \mathrm{Jul}$ & 1 & & & & & \\
\hline $20 \mathrm{Jul}$ & & & 1 & & & \\
\hline Totals & $(116)$ & $(46)$ & 46 & $21 \quad(23)$ & 34 & 20 \\
\hline
\end{tabular}


Appendix Table 7B. Detections of PIT-tagged smolts by date at three Columbia River dams for wild chinook salmon from Marsh Creek. 1995. Numbers in parentheses are first detections at the dams that have been adjusted for spill.

\begin{tabular}{|c|c|c|c|c|c|c|c|c|c|c|c|c|c|c|}
\hline \multirow[b]{2}{*}{$\begin{array}{l}\text { Detection } \\
\text { date }\end{array}$} & \multicolumn{4}{|c|}{ MCNary } & \multicolumn{5}{|c|}{$\frac{\text { John Day }}{\text { Previous detections }}$} & \multicolumn{5}{|c|}{$\frac{\text { Bonneville }}{\text { Previous detections }}$} \\
\hline & $\begin{array}{c}\text { First } \\
\text { detection }\end{array}$ & $\begin{array}{c}\frac{p r e v 1}{1} \\
\text { dam }\end{array}$ & 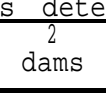 & \multirow{2}{*}{$\begin{array}{c}\frac{\frac{110 n s}{3}}{3} \\
\text { dams }\end{array}$} & $\begin{array}{c}\text { First } \\
\text { detection }\end{array}$ & \multirow{2}{*}{$\frac{1}{d a m}$} & $\frac{\frac{\text { evious }}{2}}{\text { dams }}$ & \multirow{2}{*}{$\begin{array}{c}\frac{\text { etect } 1}{3} \\
\text { dars }\end{array}$} & $\frac{\frac{15}{4}}{\text { dams }}$ & $\begin{array}{c}\text { First } \\
\text { detection }\end{array}$ & $\begin{array}{c}1 \\
\text { dam }\end{array}$ & \multirow{2}{*}{$\begin{array}{l}\text { Previous det } \\
\frac{3}{2} \\
\text { dams dams }\end{array}$} & \multirow[t]{2}{*}{ 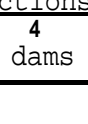 } & $\begin{array}{c}5 \\
\text { dams }\end{array}$ \\
\hline 26 Apr & & & & & & & & & & & & & & \\
\hline 29 Apr & & & & & & & & & & & & & & \\
\hline 31 May & & & & & & & & & & & & & & \\
\hline 32 May & & 1 & & & & & & & & & & & & \\
\hline 03 May & $1: 2:$ & & 1 & & & & & & & & & & & \\
\hline C4 May & & & & & & & & & & & & & & \\
\hline 09 May & & & & & & & & & & & & & & \\
\hline 10 May & & 2 & & & & & & & & & & & & \\
\hline 11 May & & & 2 & & & & & & & & & & & \\
\hline 12 May & $1 \quad$ ! 2; & & 1 & & & & & & & & & & & \\
\hline 13 May & & 1 & 1 & 2 & & & & & & & & & & \\
\hline 14 May & & 2 & & & & & & & & & & & & \\
\hline 15 May & 21 & & 1 & & & & & & & & & & & \\
\hline 16 May & & & 1 & 1 & & & & & & & & & & \\
\hline 13 May & 2: & & 1 & & & & & & & & & & & \\
\hline 13 May & & 1 & 2 & & & & & & & & & & & \\
\hline 21 May & & 1 & & & & & & & & & & & & \\
\hline 22 May & & & & & & & & & & & & & & \\
\hline 23 May & $1 ! 2$ & & & & & & & & & & & & & \\
\hline 25 May & & 1 & & & & & & & & & & & & \\
\hline 25 May & & & 2 & & & & & & & & & & & \\
\hline 28 May & $1: 2$ & & & & & & & & & & & & & \\
\hline
\end{tabular}


Appendix Table 7B. Continued.

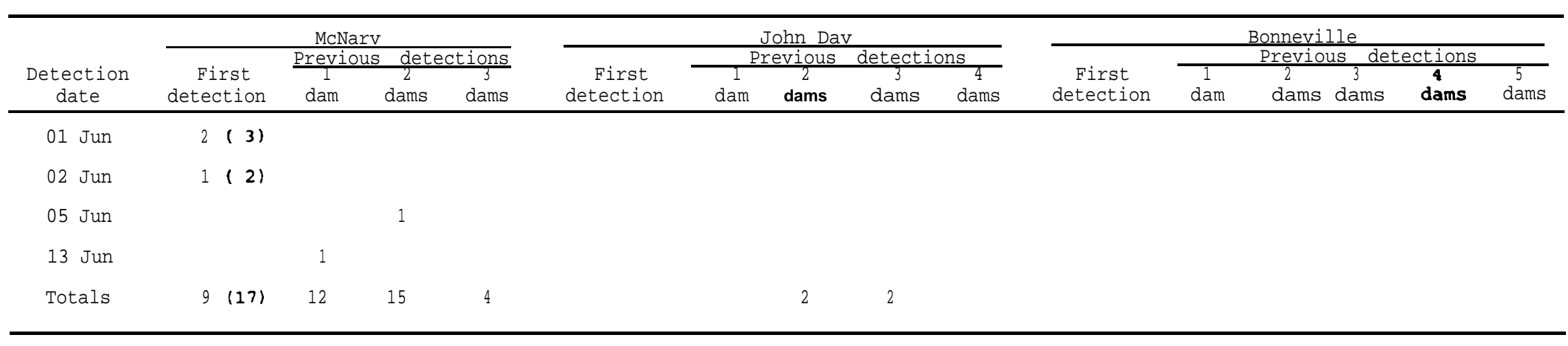


Appendix Table 8A. Detections of PIT-tagged smolts by date at three Snake River dams for wild chinook salmon from Cape Horn Creek. 1995. Numbers in parentheses are first detections at the dams that have been adjusted for spill.

Tagging site: Cape Horn Creek

Release site: Cape Horn Creek

Release river kilometer(s) above Lower Granite Dam: 635
Release date: 03 Aug - 04 Aug 1994

Number released: 1,443

\begin{tabular}{|c|c|c|c|c|c|c|}
\hline \multirow{2}{*}{$\begin{array}{c}\text { Detection } \\
\text { date }\end{array}$} & \multirow{3}{*}{$\begin{array}{c}\text { Lower Granite } \\
\text { First } \\
\text { detection }\end{array}$} & \multicolumn{2}{|c|}{ Little Goose } & \multicolumn{3}{|c|}{ Lower Monumental } \\
\hline & & \multirow[t]{2}{*}{$\begin{array}{c}\text { First } \\
\text { detection }\end{array}$} & $\begin{array}{r}\text { Previous } \\
\text { detections } \\
\text { at } 1 \text { dam }\end{array}$ & $\begin{array}{l}\text { First } \\
\text { detection }\end{array}$ & \multirow[t]{2}{*}{$\begin{array}{l}\text { Previous } \\
\text { detections } \\
\text { at } 1 \text { dam }\end{array}$} & \multirow[t]{2}{*}{$\begin{array}{l}\text { Previous } \\
\text { detections } \\
\text { at } 2 \text { dams }\end{array}$} \\
\hline 14 Apr & & & & & & \\
\hline 15 Apr & 2 & & & & & \\
\hline 18 Apr & 1 & 1 & & & & \\
\hline 22 Apr & & & 1 & & & \\
\hline 23 Apr & & & 1 & & & \\
\hline 24 Apr & 2 & & & & & \\
\hline 26 Apr & 1 & & & & & \\
\hline 27 Apr & 1 & & & & & \\
\hline 28 Apr & 1 & & 1 & & & \\
\hline 29 Apr & 1 & & & & & \\
\hline 30 Apr & & & & & & \\
\hline 01 May & 2 & & 1 & & & \\
\hline 02 May & 1 & & & & & \\
\hline 03 May & 1 & & 1 & & & \\
\hline 04 May & $3(4)$ & & & & & \\
\hline
\end{tabular}


Appendix Table 8A. Continued.

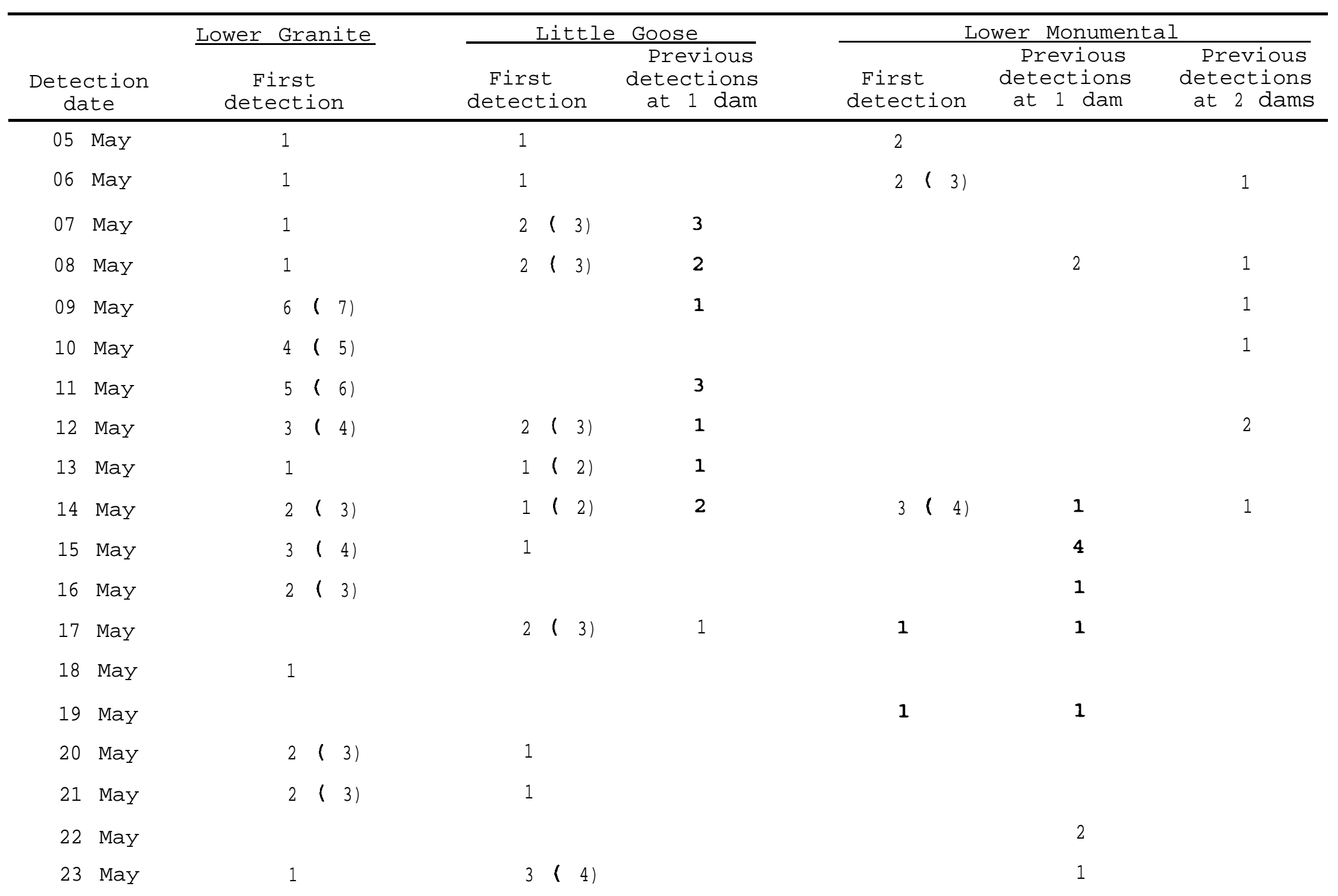


Appendix Table 8A. Continued.

\begin{tabular}{|c|c|c|c|c|c|c|}
\hline \multirow[b]{2}{*}{$\begin{array}{c}\text { Detection } \\
\text { date }\end{array}$} & \multirow{3}{*}{$\begin{array}{c}\text { Lower Granite } \\
\begin{array}{c}\text { First } \\
\text { detection }\end{array} \\
1\end{array}$} & \multicolumn{2}{|c|}{ Little Goose } & \multicolumn{3}{|c|}{ Lower Monumental } \\
\hline & & $\begin{array}{c}\text { First } \\
\text { detection }\end{array}$ & $\begin{array}{l}\text { Previous } \\
\text { detections } \\
\text { at } 1 \text { dam }\end{array}$ & $\begin{array}{l}\text { First } \\
\text { detection }\end{array}$ & $\begin{array}{l}\text { Previous } \\
\text { detections } \\
\text { at } 1 \text { dam }\end{array}$ & $\begin{array}{l}\text { Previous } \\
\text { detections } \\
\text { at } 2 \text { dams }\end{array}$ \\
\hline 24 May & & 1 & 1 & & 1 & \\
\hline 25 May & 1 & $3(4)$ & 1 & & 2 & \\
\hline 26 May & & & & & 1 & \\
\hline 27 May & 2 & & 3 & & & \\
\hline 28 May & 3 & & & & 1 & \\
\hline 29 May & 1 & & & & & \\
\hline 30 May & & & & & 1 & \\
\hline 31 May & 1 & & & & 1 & \\
\hline 01 Jun & 1 & & & & & \\
\hline 02 Jun & 2 & & & & 1 & \\
\hline 03 Jun & & & & & 3 & \\
\hline 04 Jun & & & & & & \\
\hline 05 Jun & 3 & & 1 & & & \\
\hline 06 Jun & 1 & 1 & & & 1 & \\
\hline 07 Jun & $3(4)$ & & 1 & & & \\
\hline 08 Jun & & 1 & & & 1 & \\
\hline 10 Jun & & 1 & 1 & & & 1 \\
\hline 11 Jun & & 1 & & & & \\
\hline 12 Jun & 1 & 1 & 1 & & & 1 \\
\hline
\end{tabular}


Appendix Table 8A. Continued.

\begin{tabular}{|c|c|c|c|c|c|c|}
\hline \multirow[b]{2}{*}{$\begin{array}{c}\text { Detection } \\
\text { date }\end{array}$} & \multirow{2}{*}{$\begin{array}{c}\text { Lower Granite } \\
\text { First } \\
\text { detection }\end{array}$} & \multicolumn{2}{|c|}{ Little Goose } & \multicolumn{3}{|c|}{ Lower Monumental } \\
\hline & & $\begin{array}{c}\text { First } \\
\text { detection }\end{array}$ & $\begin{array}{r}\text { Previous } \\
\text { detections } \\
\text { at } 1 \text { dam }\end{array}$ & $\begin{array}{l}\text { First } \\
\text { detection }\end{array}$ & $\begin{array}{l}\text { Previous } \\
\text { detections } \\
\text { at } 1 \text { dam }\end{array}$ & $\begin{array}{l}\text { Previous } \\
\text { detections } \\
\text { at } 2 \text { dams }\end{array}$ \\
\hline 13 Jun & & & & & & \\
\hline 14 Jun & & & & & 1 & 1 \\
\hline 16 Jun & & & & & 1 & \\
\hline 19 Jun & 2 & & & & & \\
\hline 23 Jun & 1 & 1 & & & & 1 \\
\hline 24 Jun & & 1 & & & & \\
\hline 28 Jun & & & & & 2 & \\
\hline 30 Jun & 1 & 1 & & & 1 & \\
\hline $02 \mathrm{Jul}$ & & & & 1 & & \\
\hline $03 \mathrm{Jul}$ & 1 & & & & 1 & \\
\hline $05 \mathrm{Jul}$ & 1 & & & & & \\
\hline $07 \mathrm{Jul}$ & 1 & & & & & \\
\hline $08 \mathrm{Jul}$ & & & 1 & & & \\
\hline $09 \mathrm{Jul}$ & & 1 & & 1 & & \\
\hline $10 \mathrm{Jul}$ & & & 2 & & & \\
\hline $13 \mathrm{Jul}$ & & & & & & \\
\hline $16 \mathrm{Jul}$ & & & & & & 2 \\
\hline $17 \mathrm{Jul}$ & & & 1 & & & \\
\hline $18 \mathrm{Jul}$ & & & 1 & & & \\
\hline
\end{tabular}


Appendix Table 8A. Continued.

\begin{tabular}{|c|c|c|c|c|c|c|}
\hline \multirow[b]{2}{*}{$\begin{array}{c}\text { Detection } \\
\text { date }\end{array}$} & \multirow{2}{*}{$\begin{array}{c}\text { Lower Granite } \\
\text { First } \\
\text { detection }\end{array}$} & \multicolumn{2}{|c|}{ Little Goose } & \multicolumn{3}{|c|}{ Lower Monumental } \\
\hline & & $\begin{array}{c}\text { First } \\
\text { detection }\end{array}$ & $\begin{array}{l}\text { Previous } \\
\text { detections } \\
\text { at } 1 \text { dam }\end{array}$ & $\begin{array}{l}\text { First } \\
\text { detection }\end{array}$ & $\begin{array}{l}\text { Previous } \\
\text { detections } \\
\text { at } 1 \text { dam }\end{array}$ & $\begin{array}{r}\text { Previous } \\
\text { detections } \\
\text { at } 2 \text { dams }\end{array}$ \\
\hline $22 \mathrm{Jul}$ & 1 & & & & & \\
\hline $24 \mathrm{Jul}$ & & & & & & 1 \\
\hline $25 \mathrm{Jul}$ & & & 1 & & & \\
\hline $28 \mathrm{Jul}$ & 1 & & & & & \\
\hline 02 Aug & & & 1 & & & \\
\hline 05 Aug & & & & & & \\
\hline 06 Aug & & & & & & \\
\hline 10 Aug & & 1 & & & & \\
\hline 21 Aug & & 1 & & & 1 & \\
\hline 17 Sep & & & & & 1 & \\
\hline Totals & $84 \quad(95)$ & $(47)$ & 37 & $19 \quad(21)$ & 34 & 22 \\
\hline
\end{tabular}


Appendix Table 8B. Detections of PIT-tagged smolts by date at three Columbia River dams for wild chinook salmon from Cape Horn Creek, 1995. Numbers in parentheses are first detections at the dams that have been adjusted for spill.

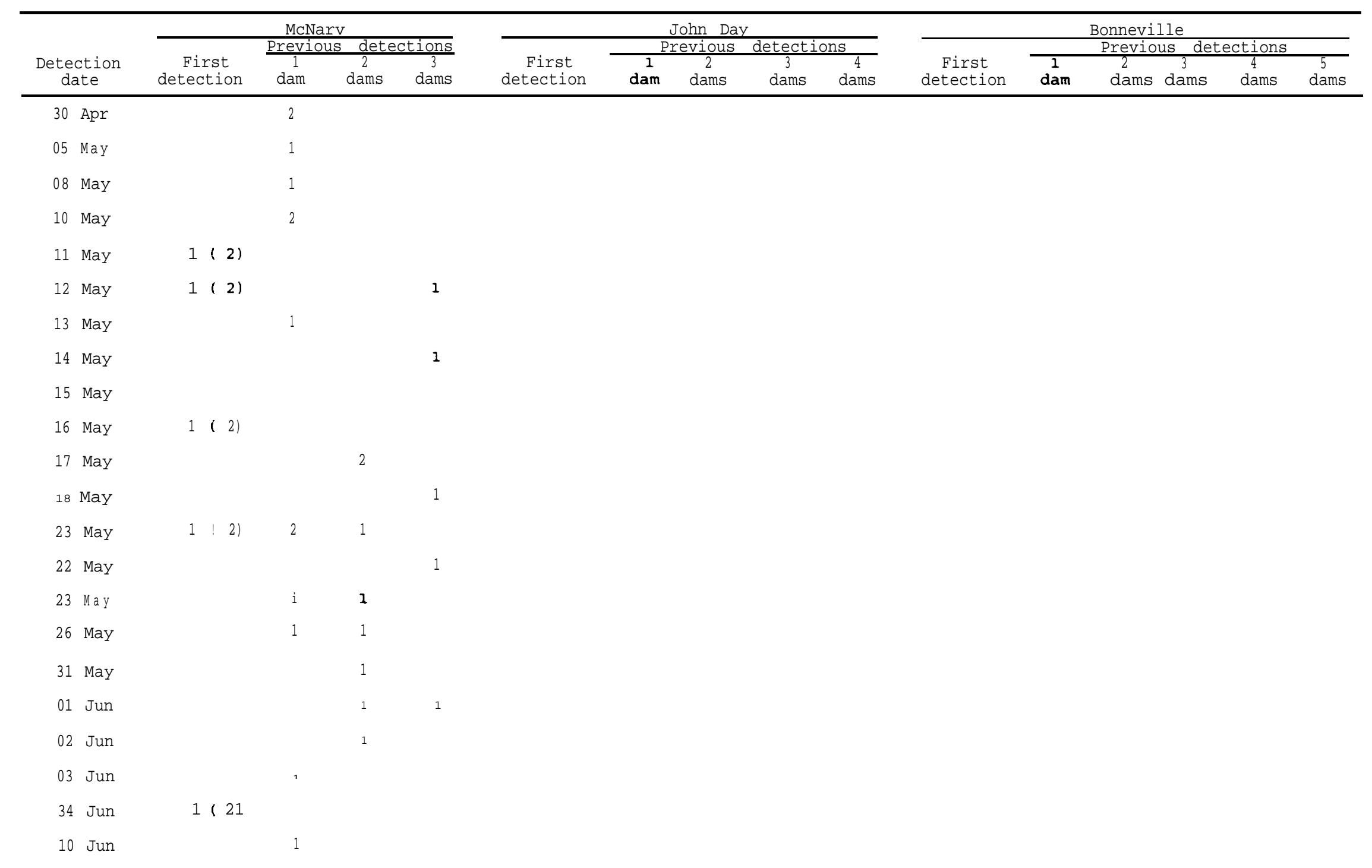


Appendix Table 8B. Continued.

\begin{tabular}{|c|c|c|c|c|c|c|c|c|c|c|c|c|c|c|c|}
\hline \multirow[b]{2}{*}{$\begin{array}{l}\text { Detection } \\
\text { date }\end{array}$} & \multicolumn{4}{|c|}{ McNarv } & \multicolumn{5}{|c|}{ John Dav } & \multicolumn{6}{|c|}{ Bonneville } \\
\hline & $\begin{array}{c}\text { First } \\
\text { detection }\end{array}$ & \multicolumn{3}{|c|}{$\frac{\text { Previous }}{1} \frac{1}{2}$} & $\begin{array}{c}\text { First } \\
\text { detection }\end{array}$ & \multicolumn{2}{|c|}{$\begin{array}{c}\text { Previous detections } \\
\end{array}$} & $\frac{\text { etect } 1}{3}$ dams & $\frac{15}{4}$ & $\begin{array}{c}\text { First } \\
\text { detection }\end{array}$ & $\begin{array}{c}1 \\
\text { dam }\end{array}$ & $\begin{array}{c}\frac{P r e v i o u}{2} \\
\text { dams }\end{array}$ & \multirow[t]{2}{*}{ 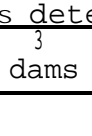 } & \multirow[t]{2}{*}{$\begin{array}{l}\frac{10 n s}{4} \\
\text { dams }\end{array}$} & $\begin{array}{c}5 \\
\text { dams }\end{array}$ \\
\hline 11 Jun & & $\mathrm{i}$ & & & & & & & & & & & & & \\
\hline 14 Jun & & & & & & & & & & & & & & & \\
\hline 15 Jun & $1(21$ & & & & & & & & & & & & & & \\
\hline 16 Jun & & & 1 & & & & & & & & & & & & \\
\hline 18 Jun & & & $I$ & & & & & & & & & & & & \\
\hline 20 Jun & & & 1 & & & & & & & & & & & & \\
\hline C2 Jul & & & 1 & & & & & & & & & & & & \\
\hline 04 Jul & & & 1 & & & & & & & & & & & & \\
\hline $14 \mathrm{Jul}$ & & 1 & & & & & & & & & & & & & \\
\hline 25 Jul 1 & & & 1 & & & & & & & & & & & & \\
\hline 31 Jul & & & & 1 & & & & & & & & & & & \\
\hline Totals & $6 \quad(12)$ & 15 & 14 & 5 & & 2 & & & & & & & & & \\
\hline
\end{tabular}


Appendix Table 9A. Detections of PIT-tagged smolts by date at three Snake River dams for wild chinook salmon from Valley Creek, 1995. Numbers in parentheses are first detections at the dams that have been adjusted for spill.

Tagging site: Valley Creek

Release date: 04 - 05 Aug 1994

Release site: Valley Creek

Number released: 1,552

Release river kilometer(s) above Lower Granite Dam: 743 - 757

\begin{tabular}{|c|c|c|c|c|c|c|c|}
\hline \multirow{2}{*}{\multicolumn{2}{|c|}{$\begin{array}{c}\text { Detection } \\
\text { date }\end{array}$}} & \multirow{2}{*}{$\begin{array}{c}\text { Lower Granite } \\
\text { First } \\
\text { detection }\end{array}$} & \multicolumn{2}{|c|}{ Little Goose } & \multicolumn{3}{|c|}{ Lower Monumental } \\
\hline & & & $\begin{array}{c}\text { First } \\
\text { detection }\end{array}$ & $\begin{array}{l}\text { Previous } \\
\text { detections } \\
\text { at } 1 \text { dam }\end{array}$ & $\begin{array}{l}\text { First } \\
\text { detection }\end{array}$ & $\begin{array}{l}\text { Previous } \\
\text { detections } \\
\text { at } 1 \text { dam }\end{array}$ & $\begin{array}{l}\text { Previous } \\
\text { detections } \\
\text { at } 2 \text { dams }\end{array}$ \\
\hline 22 & Apr & 2 & & & & & \\
\hline 28 & Apr & 1 & & & & & \\
\hline 30 & Apr & & & & & & \\
\hline 01 & May & & & 1 & & & \\
\hline 02 & May & 1 & & & & & \\
\hline 03 & May & 1 & & & & & \\
\hline 04 & May & 1 & & & & & \\
\hline 05 & May & & & & & & \\
\hline 06 & May & 1 & & 1 & & & \\
\hline 07 & May & & 1 & 1 & & & \\
\hline 09 & May & 1 & & & & & \\
\hline 10 & May & & & & 1 & & \\
\hline 11 & May & $2(3)$ & & & & 1 & \\
\hline 12 & May & & & & 1 & & \\
\hline 13 & May & & & & 1 & & \\
\hline
\end{tabular}


Appendix Table 9A. Continued.

\begin{tabular}{|c|c|c|c|c|c|c|}
\hline \multirow{2}{*}{$\begin{array}{c}\text { Detection } \\
\text { date }\end{array}$} & \multirow{3}{*}{$\begin{array}{c}\text { Lower Granite } \\
\begin{array}{c}\text { First } \\
\text { detection }\end{array} \\
1\end{array}$} & \multicolumn{2}{|c|}{ Little Goose } & \multicolumn{3}{|c|}{ Lower Monumental } \\
\hline & & \multirow[t]{2}{*}{$\begin{array}{c}\text { First } \\
\text { detection }\end{array}$} & \multirow[t]{2}{*}{$\begin{array}{r}\text { Previous } \\
\text { detections } \\
\text { at } 1 \text { dam }\end{array}$} & \multirow[t]{2}{*}{$\begin{array}{l}\text { First } \\
\text { detection }\end{array}$} & \multirow[t]{2}{*}{$\begin{array}{l}\text { Previous } \\
\text { detections } \\
\text { at } 1 \text { dam }\end{array}$} & \multirow[t]{2}{*}{$\begin{array}{l}\text { Previous } \\
\text { detections } \\
\text { at } 2 \text { dams }\end{array}$} \\
\hline 14 May & & & & & & \\
\hline 15 May & & & & & 1 & \\
\hline 16 May & 1 & & & & & \\
\hline 17 May & & & & & & \\
\hline 18 May & & & & & & \\
\hline 19 May & $2(3)$ & & & & 2 & \\
\hline 21 May & & & & & & \\
\hline 22 May & & & & & & \\
\hline 23 May & & & & & 1 & \\
\hline 24 May & & & & & & \\
\hline 25 May & & & & & & \\
\hline 26 May & & & 1 & & 1 & \\
\hline 27 May & 2 & & 1 & & 1 & \\
\hline 28 May & 1 & & & & & \\
\hline 29 May & 2 & & & & & \\
\hline 31 May & 2 & & & & & \\
\hline 01 Jun & 1 & & & & 1 & \\
\hline 02 Jun & 2 & & 2 & & & \\
\hline 03 Jun & & & 1 & & & \\
\hline
\end{tabular}


Appendix Table 9A. Continued.

\begin{tabular}{|c|c|c|c|c|c|c|}
\hline \multirow[b]{2}{*}{$\begin{array}{c}\text { Detection } \\
\text { date }\end{array}$} & \multirow{3}{*}{$\begin{array}{c}\text { Lower Granite } \\
\begin{array}{c}\text { First } \\
\text { detection }\end{array} \\
1\end{array}$} & \multicolumn{2}{|c|}{ Little Goose } & \multicolumn{3}{|c|}{ Lower Monumental } \\
\hline & & $\begin{array}{c}\text { First } \\
\text { detection }\end{array}$ & $\begin{array}{l}\text { Previous } \\
\text { detections } \\
\text { at } 1 \text { dam }\end{array}$ & $\begin{array}{l}\text { First } \\
\text { detection }\end{array}$ & $\begin{array}{l}\text { Previous } \\
\text { detections } \\
\text { at } 1 \text { dam }\end{array}$ & $\begin{array}{l}\text { Previous } \\
\text { detect.ions } \\
\text { at } 2 \text { dams }\end{array}$ \\
\hline 04 Jun & & 1 & & & & \\
\hline 06 Jun & 1 & 1 & & & 1 & \\
\hline 07 Jun & 2 & & 2 & & & \\
\hline 08 Jun & 2 & 1 & & & & \\
\hline 09 Jun & 3 & 1 & 2 & 2 & & 1 \\
\hline 10 Jun & & 1 & & & & \\
\hline 11 Jun & 1 & 1 & & & & \\
\hline 12 Jun & & 1 & 3 & & & 1 \\
\hline 13 Jun & & & & 1 & & 1 \\
\hline 14 Jun & & 2 & 1 & & & \\
\hline 15 Jun & 1 & & & & 1 & \\
\hline 16 Jun & & & & 1 & 1 & \\
\hline 17 Jun & & & & & 1 & \\
\hline 18 Jun & 1 & & & & & \\
\hline 19 Jun & 1 & & & & & 1 \\
\hline $22 J$ un & 3 & & & & & \\
\hline 23 Jun & 3 & 1 & & & 1 & \\
\hline 24 Jun & & & & & & \\
\hline 28 Jun & & 1 & 1 & & 1 & \\
\hline
\end{tabular}


Appendix Table 9A. Continued.

\begin{tabular}{|c|c|c|c|c|c|c|}
\hline \multirow[b]{2}{*}{$\begin{array}{c}\text { Detection } \\
\text { date }\end{array}$} & \multirow{2}{*}{$\begin{array}{c}\text { Lower Granite } \\
\text { First } \\
\text { detection }\end{array}$} & \multicolumn{2}{|c|}{ Little Goose } & \multicolumn{3}{|c|}{ Lower Monumental } \\
\hline & & $\begin{array}{c}\text { First } \\
\text { detection }\end{array}$ & $\begin{array}{l}\text { Previous } \\
\text { detections } \\
\text { at } 1 \text { dam }\end{array}$ & $\begin{array}{l}\text { First } \\
\text { detection }\end{array}$ & $\begin{array}{l}\text { Previous } \\
\text { detections } \\
\text { at } 1 \text { dam }\end{array}$ & $\begin{array}{l}\text { Previous } \\
\text { detections } \\
\text { at } 2 \text { dams }\end{array}$ \\
\hline 29 Jun & & 1 & & & & \\
\hline 30 Jun & & & 1 & & 1 & 1 \\
\hline & & 1 & & & & \\
\hline $02 \mathrm{Jul}$ & & & & & 1 & \\
\hline $03 \mathrm{Jul}$ & & & & & 1 & \\
\hline 05 Jui & & & & & 1 & \\
\hline $06 \mathrm{Jul}$ & & & & & 1 & \\
\hline $08 \mathrm{Jul}$ & 2 & & & & & \\
\hline 09 Jul & 1 & & & & & 1 \\
\hline $10 \mathrm{Jul}$ & & & & & & \\
\hline $11 \mathrm{Jul}$ & 2 & & & & & \\
\hline $13 \mathrm{Jul}$ & & & & & & \\
\hline $15 J$ ul & 1 & & & & & \\
\hline $18 \mathrm{Jul}$ & 1 & & 1 & & & \\
\hline $20 \mathrm{Jul}$ & & & 1 & & & \\
\hline $24 \mathrm{Jul}$ & & & 1 & & & \\
\hline $28 \mathrm{Jul}$ & & & 1 & & & 1 \\
\hline $29 \mathrm{Jul}$ & & & & & & 1 \\
\hline 04 Aug & & & & & & 1 \\
\hline
\end{tabular}


Appendix Table 9A. Continued.

\begin{tabular}{|c|c|c|c|c|c|c|}
\hline \multirow[b]{2}{*}{$\begin{array}{c}\text { Detection } \\
\text { date }\end{array}$} & \multirow{2}{*}{$\begin{array}{c}\text { Lower Granite } \\
\text { First } \\
\text { detection }\end{array}$} & \multicolumn{2}{|c|}{ Little Goose } & \multicolumn{3}{|c|}{ Lower Monumental } \\
\hline & & $\begin{array}{c}\text { First } \\
\text { detection }\end{array}$ & $\begin{array}{c}\text { Previous } \\
\text { detections } \\
\text { at } 1 \text { dam }\end{array}$ & $\begin{array}{l}\text { First } \\
\text { detection }\end{array}$ & $\begin{array}{l}\text { Previous } \\
\text { detections } \\
\text { at } 1 \text { dam }\end{array}$ & $\begin{array}{l}\text { Previous } \\
\text { detections } \\
\text { at } 2 \text { dams }\end{array}$ \\
\hline 07 Sep & & 1 & & & & \\
\hline Totals & $50 \quad(52)$ & 24 & 23 & 19 & 21 & 10 \\
\hline
\end{tabular}


Appendix Table 9B. Detections of PIT-tagged smolts by date at three Columbia River dams for wild chinook salmon from Valley Creek. 1995. Numbers in parentheses are first detections at the dams that have been adjusted for spill.

\begin{tabular}{|c|c|c|c|c|c|c|c|c|c|c|c|c|c|c|c|}
\hline \multirow{3}{*}{$\begin{array}{c}\text { Detection } \\
\text { daze }\end{array}$} & \multicolumn{4}{|c|}{ McNary } & \multirow{2}{*}{\multicolumn{5}{|c|}{$\begin{array}{l}\text { John Day } \\
\text { Previous detections }\end{array}$}} & \multirow{2}{*}{\multicolumn{6}{|c|}{$\begin{array}{l}\text { Bonneville } \\
\text { Previous detections }\end{array}$}} \\
\hline & \multirow{2}{*}{$\begin{array}{c}\text { First } \\
\text { detection }\end{array}$} & \multirow{2}{*}{\multicolumn{3}{|c|}{$\frac{\text { Previous dezections }}{\text { dam dams dams }}$}} & & & & & & & & & & & \\
\hline & & & & & $\begin{array}{c}\text { Firs: } \\
\text { detection }\end{array}$ & $d z l m$ & $\stackrel{2}{\text { dams }}$ & \multicolumn{2}{|c|}{$\begin{array}{cc}\text { detections } & \\
3 & 4 \\
\text { dams } & \text { dams }\end{array}$} & $\begin{array}{c}\text { First } \\
\text { detection }\end{array}$ & darn: & \multicolumn{2}{|c|}{$\begin{array}{l}\text { revious det } \\
2 \\
\text { dams dams }\end{array}$} & \multirow[t]{2}{*}{$\begin{array}{c}4 \\
\text { dams }\end{array}$} & $\begin{array}{c}5 \\
\text { dams } \\
\end{array}$ \\
\hline 06 May & & 1 & & & & & & & & & & & & & \\
\hline c? Yay & & : & & & & & & & & & & & & & \\
\hline $8 \mathrm{May}$ & & & 2 & & & & & & & & & & & & \\
\hline 15 may & $1 i 2:$ & & & & & & & & & & & & & & \\
\hline 17 may & & $\dot{z}$ & & & & & & & & & & & & & \\
\hline 22 may & & $=$ & & & & & & & & & & & & & \\
\hline 25 may & & 1 & & & & & & & & & & & & & \\
\hline 27 may & & 1 & & & & & & & & & & & & & \\
\hline 31 may & & 1 & & & & & & & & & & & & & \\
\hline $22 \mathrm{Jun}$ & & & - & & & & & & & & & & & & \\
\hline$=0 \mathrm{Jur}$ & & 1 & & & & & & & & & & & & & \\
\hline \multicolumn{16}{|l|}{$12 j u n$} \\
\hline 17 Jun & & 1 & & & & & & & & & & & & & \\
\hline 19 Jun & & & $\therefore$ & & & & & & & & & & & & \\
\hline 20 Jun & & & & 1 & & & & & & & & & & & \\
\hline 22 Jun & & & - & & & & & & & & & & & & \\
\hline 32 Jul & & & - & & & & & & & & & & & & \\
\hline $03 \mathrm{Jul}$ & & & & 1 & & & & & & & & & & & \\
\hline $36 \mathrm{Ju} ?$ & & 1 & $:$ & & & & & & & & & & & & \\
\hline \multicolumn{16}{|l|}{$07 \mathrm{Jul}$} \\
\hline $06 \mathrm{~J} \mathrm{ul}$ & & 1 & & & & & & & & & & & & & \\
\hline $12 \mathrm{Jul}$ & & & 1 & & & & & & & & & & & & \\
\hline
\end{tabular}


Appendix Table 9B. Continued.

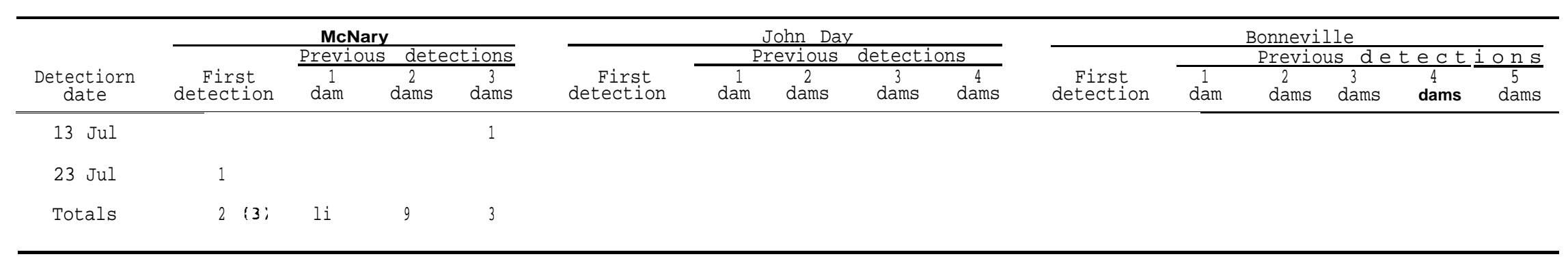


Appendix Table 10A. Detections of PIT-tagged smolts by date at three Snake River dams for wild chinook salmon from Camas Creek, 1995. Numbers in parentheses are first detections at the dams that have been adjusted for spill.

Tagging site: Camas Creek

Release date: 09 - 10 Aug 1994

Release site: Camas Creek

Number released: 1,528

Release river kilometer(s) above Lower Granite Dam: 526 - 529

\begin{tabular}{|c|c|c|c|c|c|c|c|}
\hline \multirow{2}{*}{\multicolumn{2}{|c|}{$\begin{array}{c}\text { Detection } \\
\text { date }\end{array}$}} & \multirow{3}{*}{$\begin{array}{c}\text { ower Granite } \\
\text { First } \\
\text { detection } \\
1\end{array}$} & \multicolumn{2}{|c|}{ Little Goose } & \multicolumn{3}{|c|}{ Lower Monumental } \\
\hline & & & $\begin{array}{l}\text { First } \\
\text { detection }\end{array}$ & \multirow[t]{2}{*}{$\begin{array}{l}\text { Previous } \\
\text { detections } \\
\text { at } 1 \text { dam }\end{array}$} & \multirow[t]{2}{*}{$\begin{array}{c}\text { First } \\
\text { detection }\end{array}$} & \multirow[t]{2}{*}{$\begin{array}{l}\text { Previous } \\
\text { detections } \\
\text { at } 1 \text { dam }\end{array}$} & \multirow[t]{2}{*}{$\begin{array}{l}\text { Previous } \\
\text { detections } \\
\text { at } 2 \text { dams }\end{array}$} \\
\hline 17 & Apr & & & & & & \\
\hline 20 & Apr & 1 & & & & & \\
\hline 21 & Apr & 1 & & & & & \\
\hline 25 & Apr & & & 1 & & & \\
\hline & Apr & 2 & & & 1 & & \\
\hline 27 & Apr & 2 & & & & & \\
\hline 29 & Apr & & & & 1 & & \\
\hline 30 & Apr & 2 & & & & & \\
\hline 01 & May & & & & & 1 & \\
\hline 0 & May & 2 & 1 & 1 & & 1 & \\
\hline 03 & May & 3 & & 1 & & 2 & \\
\hline 04 & May & 1 & 1 & & 1 & 1 & \\
\hline 06 & May & & $2(3)$ & & & 1 & \\
\hline 07 & May & 1 & & & $2(3)$ & 1 & \\
\hline 08 & May & 1 & 1 & & & 1 & \\
\hline
\end{tabular}


Appendix Table 1 OA. Continued.

\begin{tabular}{|c|c|c|c|c|c|c|c|}
\hline \multirow{2}{*}{\multicolumn{2}{|c|}{$\begin{array}{c}\text { Detection } \\
\text { date }\end{array}$}} & \multirow{2}{*}{$\begin{array}{c}\text { Lower Granite } \\
\text { First } \\
\text { detection }\end{array}$} & \multicolumn{2}{|c|}{ Little Goose } & \multicolumn{3}{|c|}{ Lower Monumental } \\
\hline & & & $\begin{array}{c}\text { First } \\
\text { detection }\end{array}$ & \multirow{2}{*}{$\begin{array}{r}\text { Previous } \\
\text { detections } \\
\text { at } 1 \text { dam } \\
1\end{array}$} & $\begin{array}{l}\text { First } \\
\text { detection }\end{array}$ & \multirow[t]{2}{*}{$\begin{array}{l}\text { Previous } \\
\text { detections } \\
\text { at } 1 \text { dam }\end{array}$} & \multirow[t]{2}{*}{$\begin{array}{l}\text { Previous } \\
\text { detections } \\
\text { at } 2 \text { dams }\end{array}$} \\
\hline 09 & May & $5(6)$ & $2(3)$ & & & & \\
\hline 10 & May & 2 & & & & 1 & \\
\hline 11 & May & $5(6)$ & 1 & 2 & & 1 & \\
\hline 12 & May & $3(4)$ & $2(3)$ & & 1 & & \\
\hline 13 & May & 1 & & 1 & 1 & 2 & \\
\hline 14 & May & $3(4)$ & $2(3)$ & & 2 & & \\
\hline 15 & May & & 1 & & & & \\
\hline 16 & May & & $2(3)$ & & 1 & & \\
\hline 17 & May & & $2(3)$ & & $3(4)$ & 4 & \\
\hline 18 & May & & & & & 2 & \\
\hline 19 & May & 1 & $2(3)$ & 1 & 1 & 1 & \\
\hline 20 & May & 1 & & & 1 & & \\
\hline 21 & May & & & & 1 & & \\
\hline 22 & May & & & & & & \\
\hline 23 & May & $2(3)$ & & & 1 & & \\
\hline 24 & May & $3(4)$ & & & & 1 & \\
\hline 25 & May & 2 & 1 & & & & 1 \\
\hline 26 & May & & & & & 1 & \\
\hline 27 & May & 2 & 2 & & & & \\
\hline
\end{tabular}


Appendix Table 1 OA. Continued.

\begin{tabular}{|c|c|c|c|c|c|c|}
\hline \multirow{2}{*}{$\begin{array}{l}\text { Detection } \\
\text { date }\end{array}$} & \multirow{3}{*}{ 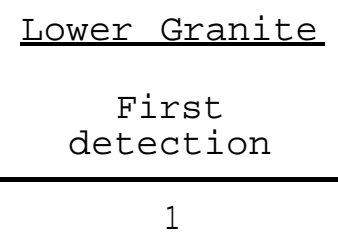 } & \multicolumn{2}{|c|}{ Little Goose } & \multicolumn{3}{|c|}{ Lower Monumental } \\
\hline & & $\begin{array}{c}\text { First } \\
\text { detection }\end{array}$ & $\begin{array}{l}\text { Previous } \\
\text { detections } \\
\text { at } 1 \text { dam }\end{array}$ & \multirow{2}{*}{$\begin{array}{c}\begin{array}{c}\text { First } \\
\text { detection }\end{array} \\
1\end{array}$} & \multirow{2}{*}{$\begin{array}{c}\text { Previous } \\
\text { detections } \\
\text { at } 1 \text { dam } \\
1\end{array}$} & \multirow[t]{2}{*}{$\begin{array}{l}\text { Previous } \\
\text { detections } \\
\text { at } 2 \text { dams }\end{array}$} \\
\hline 28 May & & & 1 & & & \\
\hline 29 May & 3 & & 1 & & & \\
\hline 30 May & 1 & & 1 & & 1 & \\
\hline 31 May & & 1 & & 2 & & \\
\hline 01 Jun & & 1 & 1 & & & \\
\hline 02 Jun & & & & & & 1 \\
\hline 03 Jun & 1 & 1 & 1 & & & \\
\hline 04 Jun & & & & & 1 & \\
\hline 05 Jun & 1 & & 1 & 1 & 1 & \\
\hline 06 Jun & $3(4)$ & & 1 & & & \\
\hline 07 Jun & & $1(2)$ & & & & \\
\hline 08 Jun & & 2 & 3 & & & \\
\hline 09 Jun & & & & 1 & 1 & \\
\hline 10 Jun & & & 1 & & 1 & 1 \\
\hline 11 Jun & 2 & 2 & 1 & & 1 & \\
\hline 12 Jun & & & & & & 1 \\
\hline 14 Jun & & & & & 1 & \\
\hline 15 Jun & & & 1 & & & 1 \\
\hline 16 Jun & & 1 & & & & \\
\hline
\end{tabular}


Appendix Table 1 OA. Continued.

\begin{tabular}{|c|c|c|c|c|c|c|}
\hline \multirow[b]{2}{*}{$\begin{array}{c}\text { Detection } \\
\text { date }\end{array}$} & \multirow{2}{*}{$\begin{array}{c}\text { Lower Granite } \\
\text { First } \\
\text { detection }\end{array}$} & \multicolumn{2}{|c|}{ Little Goose } & \multicolumn{3}{|c|}{ Lower Monumental } \\
\hline & & $\begin{array}{c}\text { First } \\
\text { detection }\end{array}$ & $\begin{array}{c}\text { Previous } \\
\text { detections } \\
\text { at } 1 \text { dam }\end{array}$ & $\begin{array}{l}\text { First } \\
\text { detection }\end{array}$ & $\begin{array}{l}\text { Previous } \\
\text { detections } \\
\text { at } 1 \text { dam }\end{array}$ & $\begin{array}{l}\text { Previxs } \\
\text { detections } \\
\text { at } 2 \text { dams }\end{array}$ \\
\hline 17 Jun & & & & & 1 & \\
\hline 18 Jun & & & & & & 1 \\
\hline 21 Jun & & & 1 & & & \\
\hline 29 Jun & & & & & & 1 \\
\hline Totals & $59 \quad(66)$ & $32 \quad(40)$ & 23 & $25 \quad(27)$ & 31 & 9 \\
\hline
\end{tabular}


Appendix Table 10B. Detections of PIT-tagged smolts by date at three Columbia River dams for wild chinook salmon from Camas Creek. 1995. Numbers in parentheses are first detections at the dams that have been adjusted for spill.

\begin{tabular}{|c|c|c|c|c|c|c|c|c|c|c|c|c|c|c|c|}
\hline \multirow[b]{2}{*}{$\begin{array}{l}\text { Detection } \\
\text { date }\end{array}$} & \multicolumn{4}{|c|}{ MoNary } & \multicolumn{5}{|c|}{$\begin{array}{l}\text { John Day } \\
\text { Previous jetections }\end{array}$} & \multicolumn{6}{|c|}{$\frac{\text { Bonneville }}{\text { previous detections }}$} \\
\hline & $\begin{array}{l}\text { Eist } \\
\text { detection }\end{array}$ & \multirow{2}{*}{$\begin{array}{l}\frac{P r e v 1}{1} \\
\text { dam }\end{array}$} & \multirow{2}{*}{$\begin{array}{l}\frac{5 \text { jete }}{2} \\
\text { dams }\end{array}$} & \multirow{2}{*}{$\begin{array}{l}\frac{\text { t1ons }}{3} \\
\text { dams }\end{array}$} & $\begin{array}{c}\text { First } \\
\text { detection }\end{array}$ & \multirow{2}{*}{$\frac{1}{d a m}$} & \multirow{2}{*}{$\begin{array}{l}\frac{e_{10 u s}}{2} \\
\text { dams }\end{array}$} & \multirow{2}{*}{$\begin{array}{l}3 \\
\text { dams }\end{array}$} & \multirow{2}{*}{$\begin{array}{c}4 \\
\text { dams }\end{array}$} & $\begin{array}{c}\text { First } \\
\text { detecticn }\end{array}$ & \multirow{2}{*}{$\begin{array}{l}1 \\
\text { dar. }\end{array}$} & \multirow{2}{*}{\multicolumn{3}{|c|}{$\begin{array}{ccc}\text { Previous } & \text { detections } \\
2 & 3 & 4 \\
\text { dams } & \text { dams } & \text { dams }\end{array}$}} & $\begin{array}{c}5 \\
\text { dams }\end{array}$ \\
\hline 03 May & & & & & & & & & & & & & & & \\
\hline 35 May & 2 & 1 & & & & & & & & & & & & & \\
\hline 08 may & & & - & & & & & & & & & & & & \\
\hline 11 may & & 2 & & & & & & & & & & & & & \\
\hline 13 may & & & & & & & & & & & & & & & \\
\hline $15 \mathrm{Yay}_{\mathbf{y}}$ & $: \quad i z:$ & & & & & & & & & & & & & & \\
\hline 16 may & & - & & & & & & & & & & & & & \\
\hline 17 may & & & 1 & & & & & & & & & & & & \\
\hline 18 ma $y$ & $1: 2:$ & & 1 & 1 & & & & & & & & & & & \\
\hline 19 may & & & & & & & & & & & & & & & \\
\hline 21 may & & & & & & & & & & & & & & & \\
\hline 22 may & 2 & & & & & & & & & & & & & & \\
\hline 23 Kay & 4 & & & & & & & & & & & & & & \\
\hline 30 may & & & & & & & & & & & & & & & \\
\hline $31 \mathrm{kay}$ & & 1 & & & & & & & & & & & & & \\
\hline 31 Zur. & & & & - & & & & & & & & & & & \\
\hline 02 jun & & & - & & & & & & & & & & & & \\
\hline $05 \mathrm{Jun}$ & & & & & & & & & & & & & & & \\
\hline CR Jun & & & & & & & & & & & & & & & \\
\hline Totals & $1 \quad(12)$ & 10 & 7 & 2 & & 1 & & & & & & & & & \\
\hline
\end{tabular}


Appendix Table 1 IA. Detections of PIT-tagged smolts by date at three Snake River dams for wild chinook salmon from Loon Creek, 1995. Numbers in parentheses are first detections at the dams that have been adjusted for spill.

Tagging site: Loon Creek

Release date: 09 Aug 1994

Release site: Loon Creek

Number released: 964

Release river kilometer(s) above Lower Granite Dam: 555 - 557

\begin{tabular}{|c|c|c|c|c|c|c|}
\hline \multirow[b]{2}{*}{$\begin{array}{c}\text { Detection } \\
\text { date }\end{array}$} & \multirow{3}{*}{$\begin{array}{c}\text { Lower Granite } \\
\begin{array}{c}\text { First } \\
\text { detection }\end{array} \\
2\end{array}$} & \multicolumn{2}{|c|}{ Little Goose } & \multicolumn{3}{|c|}{ Lower Monumental } \\
\hline & & $\begin{array}{c}\text { First } \\
\text { detection }\end{array}$ & $\begin{array}{l}\text { Previous } \\
\text { detections } \\
\text { at } 1 \text { dam }\end{array}$ & $\begin{array}{l}\text { First } \\
\text { detection }\end{array}$ & $\begin{array}{l}\text { Previous } \\
\text { detections } \\
\text { at } 1 \text { dam }\end{array}$ & $\begin{array}{l}\text { Previous } \\
\text { detections } \\
\text { at } 2 \text { dams }\end{array}$ \\
\hline 13 Apr & & & & & & \\
\hline 16 Apr & 1 & & & & & \\
\hline 17 Apr & & & 1 & & & \\
\hline 18 Apr & 1 & & & & & \\
\hline 19 Apr & 2 & & & & & \\
\hline 20 Apr & 2 & & & & & \\
\hline 21 Apr & & & & & & \\
\hline 22 Apr & 1 & & & & & \\
\hline 23 Apr & 1 & & & & & \\
\hline 24 Apr & & & & 1 & & \\
\hline 27 Apr & 3 & & & & & \\
\hline 28 Apr & 3 & & & & & \\
\hline 29 Apr & 4 & & & & & \\
\hline 01 May & 1 & & & & & \\
\hline 02 May & 1 & & & & & \\
\hline
\end{tabular}


Appendix Table I 1A. Continued.

\begin{tabular}{|c|c|c|c|c|c|c|c|}
\hline \multirow{2}{*}{\multicolumn{2}{|c|}{$\begin{array}{l}\text { Detection } \\
\text { date }\end{array}$}} & \multirow{2}{*}{$\begin{array}{c}\text { Lower Granite } \\
\text { First } \\
\text { detection }\end{array}$} & \multicolumn{2}{|c|}{ Little Goose } & \multicolumn{3}{|c|}{ Lower Monumental } \\
\hline & & & $\begin{array}{c}\text { First } \\
\text { detection }\end{array}$ & $\begin{array}{r}\text { Previous } \\
\text { detections } \\
\text { at } 1 \text { dam }\end{array}$ & $\begin{array}{l}\text { First } \\
\text { detection }\end{array}$ & $\begin{array}{l}\text { Previous } \\
\text { detections } \\
\text { at } 1 \text { dam }\end{array}$ & \multirow[t]{2}{*}{$\begin{array}{l}\text { Previous } \\
\text { detections } \\
\text { at } 2 \text { dams }\end{array}$} \\
\hline 03 & May & 3 & $2(3)$ & 1 & 1 & & \\
\hline 04 & May & $3(4)$ & $2(3)$ & 1 & 1 & & 1 \\
\hline 05 & May & & 1 & 1 & 1 & 1 & \\
\hline 06 & May & & & & 1 & 2 & \\
\hline 07 & May & & $4(6)$ & 1 & & 2 & \\
\hline 08 & May & 1 & $2(3)$ & & & 1 & \\
\hline 09 & May & $8 \quad(10)$ & & & & 2 & \\
\hline 10 & May & \begin{tabular}{l|l}
4 & $(5)$
\end{tabular} & 1 & & & 3 & \\
\hline 11 & May & $\begin{array}{lll}4 & 51\end{array}$ & $3(4)$ & & 1 & & \\
\hline 12 & May & $4(5)$ & 1 & & 2 & 1 & \\
\hline 13 & May & $6(7)$ & $2(3)$ & 1 & $3(4)$ & 1 & \\
\hline 14 & May & $3(4)$ & $2(3)$ & & 1 & 3 & \\
\hline 15 & May & & $3(4)$ & 1 & 1 & 2 & \\
\hline 16 & May & $2(3)$ & $2(3)$ & 2 & 2 & 1 & \\
\hline 17 & May & $2(3)$ & 1 & 1 & & 3 & \\
\hline 18 & May & $2(3)$ & 1 & 1 & $3(4)$ & 4 & \\
\hline 19 & May & & 1 & 2 & & & \\
\hline 20 & May & & $2(3)$ & 3 & 1 & 1 & \\
\hline 21 & May & & & 1 & $3(4)$ & & \\
\hline
\end{tabular}


Appendix Table I 1 A. Continued.

\begin{tabular}{|c|c|c|c|c|c|c|}
\hline \multirow[b]{2}{*}{$\begin{array}{c}\text { Detection } \\
\text { date }\end{array}$} & \multirow{2}{*}{$\begin{array}{c}\text { Lower Granite } \\
\text { First } \\
\text { detection }\end{array}$} & \multicolumn{2}{|c|}{ Little Goose } & \multicolumn{3}{|c|}{ Lower Monumental } \\
\hline & & $\begin{array}{c}\text { First } \\
\text { detection }\end{array}$ & $\begin{array}{l}\text { Previous } \\
\text { detections } \\
\text { at } 1 \text { dam }\end{array}$ & $\begin{array}{l}\text { First } \\
\text { detection }\end{array}$ & $\begin{array}{l}\text { Previous } \\
\text { detections } \\
\text { at } 1 \text { dam }\end{array}$ & $\begin{array}{l}\text { Previous } \\
\text { detections } \\
\text { at } 2 \text { dams }\end{array}$ \\
\hline 22 May & & & 2 & & 2 & 1 \\
\hline 23 May & 1 & & & & 1 & 1 \\
\hline 24 May & 1 & $2(3)$ & & & 1 & \\
\hline 25 May & & & & & 1 & \\
\hline 26 May & $3(4)$ & 1 & & & & \\
\hline 27 May & 2 & & & & & \\
\hline 28 May & 3 & 1 & & & 1 & \\
\hline 29 May & 2 & & & & & \\
\hline 30 May & & 2 & & & & \\
\hline 31 May & & 1 & & & & \\
\hline 01 Jun & 2 & & & & 1 & \\
\hline 02 Jun & & 1 & 2 & & & \\
\hline 03 Jun & 3 & 1 & 2 & & & \\
\hline 04 Jun & & 1 & & & & \\
\hline 05 Jun & 1 & & & & 1 & \\
\hline 06 Jun & & & & & & \\
\hline 07 Jun & 1 & & 1 & & 1 & \\
\hline 08 Jun & & & 1 & & & \\
\hline 09 Jun & & & & & 1 & \\
\hline
\end{tabular}


Appendix Table 11A. Continued.

\begin{tabular}{|c|c|c|c|c|c|c|}
\hline \multirow[b]{2}{*}{$\begin{array}{c}\text { Detection } \\
\text { date }\end{array}$} & \multirow{2}{*}{$\begin{array}{c}\text { Lower Granite } \\
\text { First } \\
\text { detection }\end{array}$} & \multicolumn{2}{|c|}{ Little Goose } & \multicolumn{3}{|c|}{ Lower Monumental } \\
\hline & & $\begin{array}{c}\text { First } \\
\text { detection }\end{array}$ & $\begin{array}{r}\text { Previous } \\
\text { detections } \\
\text { at } 1 \text { dam }\end{array}$ & $\begin{array}{l}\text { First } \\
\text { detection }\end{array}$ & $\begin{array}{l}\text { Previous } \\
\text { detections } \\
\text { at } 1 \text { dam }\end{array}$ & $\begin{array}{l}\text { Previous } \\
\text { detections } \\
\text { at } 2 \text { dams }\end{array}$ \\
\hline 10 Jun & & & 2 & & & \\
\hline 11 Jun & & & 2 & & & 1 \\
\hline 12 Jun & & & & 2 & & \\
\hline 14 Jun & & & & & & 1 \\
\hline 15 Jun & & & & & 1 & 1 \\
\hline 16 Jun & & & & & & 1 \\
\hline 23 Sun & & & & & & 1 \\
\hline 24 Jun & & & & 1 & & \\
\hline Totals & $83 \quad(95)$ & (52) & 36 & $30 \quad(33)$ & 38 & 16 \\
\hline
\end{tabular}


Appendix Table I IB. Detections of PIT-tagged smolts by date at three Columbia River dams for wild chinook salmon from Loon Creek. 1995. Numbers in parentheses are first detections at the dams that have been adjusted for spill.

\begin{tabular}{|c|c|c|c|c|c|c|c|c|c|c|c|c|c|c|c|}
\hline \multirow{3}{*}{$\begin{array}{c}\text { Detection } \\
\text { date }\end{array}$} & \multicolumn{4}{|c|}{ McNary } & \multicolumn{5}{|c|}{ John Day } & \multirow{2}{*}{\multicolumn{6}{|c|}{$\frac{\text { Bonneville }}{\text { Previous detections }}$}} \\
\hline & \multirow{2}{*}{$\begin{array}{c}\text { Firs: } \\
\text { detection }\end{array}$} & \multicolumn{3}{|c|}{ Previous getections } & \multirow{2}{*}{$\begin{array}{c}\text { First } \\
\text { detection }\end{array}$} & \multicolumn{2}{|c|}{$\begin{array}{l}\frac{\text { Previous }}{2} \\
1 \frac{2}{2}\end{array}$} & \multirow{3}{*}{$\begin{array}{l}\frac{\text { etect 1 }}{3} \\
\text { dams }\end{array}$} & \multirow{2}{*}{$\begin{array}{l}\frac{4}{4} \\
\text { dams }\end{array}$} & & & & & & \\
\hline & & dam & dams & dams & & dam & dams & & & $\begin{array}{c}\text { First } \\
\text { detection }\end{array}$ & $\frac{1}{d a m}$ & $\begin{array}{l}\frac{y e v i o l}{2} \\
\text { dams }\end{array}$ & \multirow{2}{*}{$\begin{array}{l}\frac{s \text { det }}{3} \\
\text { dams }\end{array}$} & \multirow{2}{*}{$\begin{array}{l}\frac{\text { tions }}{4} \\
\text { dams }\end{array}$} & $\begin{array}{l}5 \\
\text { dams }\end{array}$ \\
\hline $30 \mathrm{Apr}$ & & & i & & & & & & & & & & & & \\
\hline 07 May & & & 1 & & & & & & & & & & & & \\
\hline 08 May & & & & 1 & & & & & & & & & & & \\
\hline 09 May & & & - & & & & & & & & & & & & \\
\hline 10 May & & & 1 & & & & & & & & & & & & \\
\hline 12 May & & 1 & 2 & & & & & & & & & & & & \\
\hline 13 May & & & 2 & & & & & & & & & & & & \\
\hline 15 May & & & 1 & & & & & & & & & & & & \\
\hline 16 May & & 1 & & & & & & & & & & & & & \\
\hline 17 MAY & & 1 & $=$ & & & & & & & & & & & & \\
\hline 18 May & & & 2 & & & & & & & & & & & & \\
\hline 19May & & 2 & 1 & & & & & & & & & & & & \\
\hline 20 May & & 1 & : & & & & & & & & & & & & \\
\hline 21 May & 12 & & & & & & & & & & & & & & \\
\hline 22 May & & & 1 & & & & & & & & & & & & \\
\hline 23 May & 1 (2) & 2 & - & & & & & & & & & & & & \\
\hline 24 May & & & 1 & & & & & & & & & & & & \\
\hline 26 May & & 1 & & 1 & & & & & & & & & & & \\
\hline 27 May & & & & 1 & & & & & & & & & & & \\
\hline 29 May & & & 1 & & & & & & & & & & & & \\
\hline 31 Jun & & 1 & 2 & & & & & & & & & & & & \\
\hline $02 \mathrm{Jun}$ & & & 1 & & & & & & & & & & & & \\
\hline
\end{tabular}


Appendix Table 1 I B. Continued.

\begin{tabular}{|c|c|c|c|c|c|c|c|c|c|c|c|c|c|c|c|}
\hline \multirow{3}{*}{$\begin{array}{l}\text { Detection } \\
\text { date }\end{array}$} & \multicolumn{4}{|c|}{ McNary } & \multicolumn{5}{|c|}{ John Day } & \multicolumn{6}{|c|}{ Bonneville } \\
\hline & \multirow{2}{*}{$\begin{array}{c}\text { First } \\
\text { detection }\end{array}$} & \multirow{2}{*}{\multicolumn{3}{|c|}{$\frac{\text { Previous detections }}{\text { dams dams }}$}} & \multirow[b]{2}{*}{$\begin{array}{c}\text { First } \\
\text { detection }\end{array}$} & \multicolumn{4}{|c|}{ Previous detections } & \multirow[b]{2}{*}{$\begin{array}{c}\text { First } \\
\text { detection }\end{array}$} & \multicolumn{5}{|c|}{ Previous detections } \\
\hline & & & & & & $\begin{array}{c}1 \\
\text { dam }\end{array}$ & $\begin{array}{c}2 \\
\text { dams }\end{array}$ & $\begin{array}{c}3 \\
\text { dams }\end{array}$ & $\begin{array}{c}4 \\
\text { dams }\end{array}$ & & $\begin{array}{c}1 \\
\text { dam }\end{array}$ & dams & $\begin{array}{c}3 \\
\text { dams }\end{array}$ & $\begin{array}{c}4 \\
\text { dams }\end{array}$ & $\begin{array}{c}5 \\
\text { dams }\end{array}$ \\
\hline 09 j ur. & & 1 & & & & & & & & & & & & & \\
\hline $19 \mathrm{Jun}$ & & 1 & & 1 & & & & & & & & & & & \\
\hline $28 \mathrm{Jun}$ & & 1 & & & & & & & & & & & & & \\
\hline Totals & 24 & 13 & 21 & 4 & & & & & & & & & & & \\
\hline
\end{tabular}


Appendix Table 12A. Detections of PIT-tagged smolts by date at three Snake River dams for wild chinook salmon from Herd Creek. 1995. Numbers in parentheses are first detections at the dams that have been adjusted for spill.

Tagging site: Herd Creek

Release date: I2 - I3 Aug 1994

Release site: Herd Creek

Number released: 534

Release river kilometer(s) above Lower Granite Dam: 697 - 698

\begin{tabular}{|c|c|c|c|c|c|c|}
\hline \multirow{2}{*}{$\begin{array}{l}\text { Detection } \\
\text { date }\end{array}$} & \multirow{3}{*}{$\begin{array}{c}\frac{\text { Lower Granite }}{\text { First }} \\
\text { detection }\end{array}$} & \multicolumn{2}{|c|}{ Little Goose } & \multicolumn{3}{|c|}{ Lower Monumental } \\
\hline & & $\begin{array}{c}\text { First } \\
\text { detection }\end{array}$ & $\begin{array}{l}\text { Previous } \\
\text { detections } \\
\text { at } 1 \text { dam }\end{array}$ & $\begin{array}{l}\text { First } \\
\text { detection }\end{array}$ & $\begin{array}{l}\text { Previous } \\
\text { detections } \\
\text { at } 1 \text { dam }\end{array}$ & $\begin{array}{l}\text { Previous } \\
\text { detections } \\
\text { at } 2 \text { dams }\end{array}$ \\
\hline 11 Apr & & & & & & \\
\hline 16 Apr & 1 & & & & & \\
\hline 17 Apr & 1 & & & & & \\
\hline 18 Apr & 2 & & & & & \\
\hline 19 Apr & 2 & & & & 1 & \\
\hline 21 Apr & 2 & & & & & \\
\hline 22 Apr & & $1(2)$ & & & & \\
\hline 24 Apr & & & 1 & & & \\
\hline 25 Apr & 2 & & & & & \\
\hline 27 Apr & 2 & & & & & \\
\hline 29 Apr & & & & & & \\
\hline 30 Apr & 2 & & & & 1 & \\
\hline 01 May & 3 & & & & & \\
\hline 02 May & & & & & 1 & \\
\hline 03 May & 2 & & & & & \\
\hline
\end{tabular}


Appendix Table 12A. Continued.

\begin{tabular}{|c|c|c|c|c|c|c|c|}
\hline & & Lower Granite & Little & Goose & & wer Monument & \\
\hline $\begin{array}{r}\text { Dete } \\
d \bar{c}\end{array}$ & $\begin{array}{l}\text { ection } \\
\text { ate }\end{array}$ & $\begin{array}{c}\text { First } \\
\text { detection }\end{array}$ & $\begin{array}{c}\text { First } \\
\text { detection, }\end{array}$ & $\begin{array}{l}\text { Previous } \\
\text { detections } \\
\text { at } 1 \text { dam }\end{array}$ & $\begin{array}{l}\text { First } \\
\text { detection }\end{array}$ & $\begin{array}{l}\text { Previous } \\
\text { detections } \\
\text { at } 1 \text { dam }\end{array}$ & $\begin{array}{l}\text { Previous } \\
\text { detections } \\
\text { at } 2 \text { dams }\end{array}$ \\
\hline 04 & May & $2(3)$ & 1 & & & 1 & 1 \\
\hline 05 & May & & $2(3)$ & & & & \\
\hline 07 & May & 2 & 1 & & 1 & 1 & \\
\hline 08 & May & & & & 1 & 2 & \\
\hline 09 & May & $4 \quad(5)$ & & & & & \\
\hline 10 & May & 2 & & & 1 & 1 & \\
\hline 11 & May & & $\mathbf{i}$ & & & & \\
\hline 13 & May & 2 & $1(2)$ & & 1 & 1 & \\
\hline 14 & May & 1 & & & & & \\
\hline 15 & May & & 1 & 1 & & 1 & \\
\hline 16 & May & & $2(3)$ & & 1 & & \\
\hline 18 & May & & & 1 & & 1 & \\
\hline 20 & May & & & & $2(3)$ & & \\
\hline 21 & May & & 1 & 1 & & & \\
\hline 23 & May & & & & & & 1 \\
\hline 27 & May & & 1 & & & & \\
\hline 28 & Kay & & 1 & & & & \\
\hline 03 & Jun & & & & & & \\
\hline 05 & Jun & & & & & & \\
\hline
\end{tabular}


Appendix Table 12A.

\begin{tabular}{|c|c|c|c|c|c|c|}
\hline \multirow[b]{2}{*}{$\begin{array}{c}\text { Detection } \\
\text { date }\end{array}$} & \multirow{2}{*}{$\begin{array}{c}\text { Lower Granite } \\
\text { First } \\
\text { detection }\end{array}$} & \multicolumn{2}{|c|}{ Little Goose } & \multicolumn{3}{|c|}{ Lower Monumental } \\
\hline & & $\begin{array}{c}\text { First } \\
\text { detection }\end{array}$ & $\begin{array}{l}\text { Previous } \\
\text { detections } \\
\text { at } 1 \text { dam }\end{array}$ & $\begin{array}{l}\text { First } \\
\text { detection }\end{array}$ & $\begin{array}{l}\text { Previous } \\
\text { detections } \\
\text { at } 1 \text { dam }\end{array}$ & $\begin{array}{l}\text { Previous } \\
\text { detections } \\
\text { at } 2 \text { dams }\end{array}$ \\
\hline 08 Jun & & & & 1 & & \\
\hline Totals & $36 \quad(38)$ & $(21)$ & 11 & (11) & 12 & 2 \\
\hline
\end{tabular}


Appendix Table 12B. Detections of PIT-tagged smolts by date at three Columbia River dams for wild chinook salmon from Herd Creek. 1995. Numbers in parentheses are first detections at the dams that have been adjusted for spill.

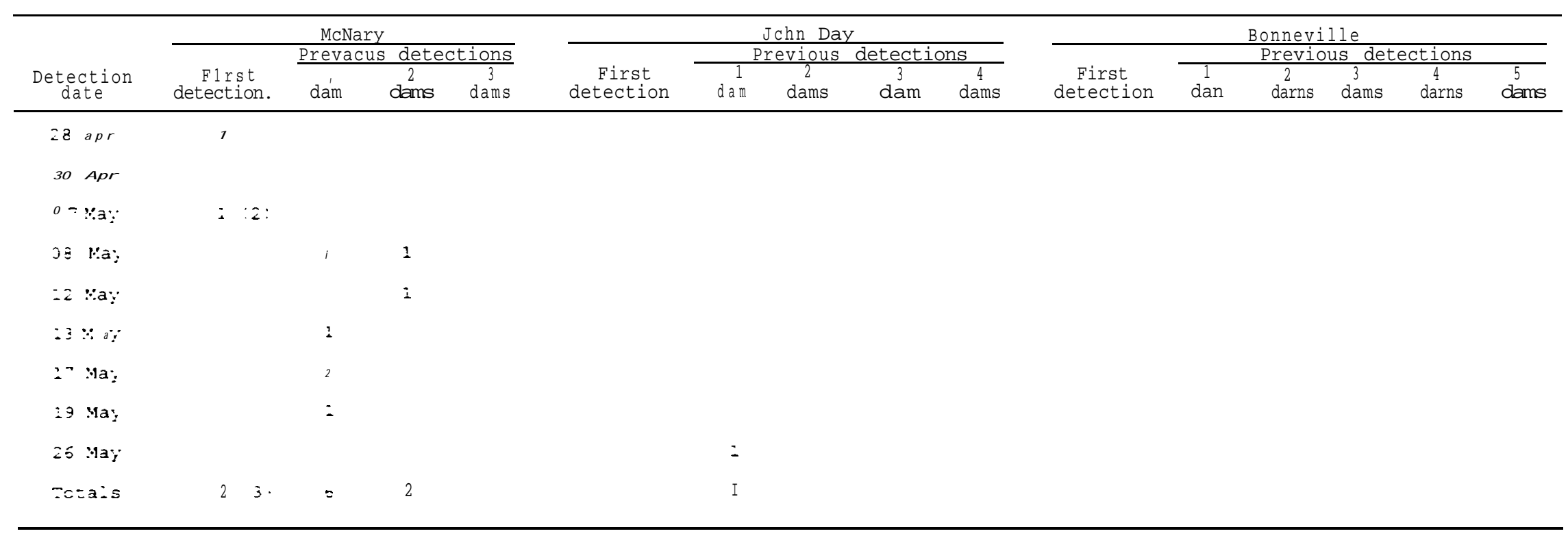


Appendix Table 13A. Detections of PIT-tagged smolts by date at three Snake River dams for wild chinook salmon from the East Fork of the Salmon River. 1995. Numbers in parentheses are first detections at the dams that have been adjusted for spill.

Tagging site: E. F. Salmon River

Release date: 12 - 13 Aug 1994

Release site: E. F. Salmon River

Number released: 986

Release river kilometer(s) above Lower Granite Dam: 695 - 698

\begin{tabular}{|c|c|c|c|c|c|c|}
\hline \multirow[b]{2}{*}{$\begin{array}{c}\text { Detection } \\
\text { date }\end{array}$} & \multirow{3}{*}{$\begin{array}{c}\text { Lower Granite } \\
\begin{array}{c}\text { First } \\
\text { detection }\end{array} \\
1\end{array}$} & \multicolumn{2}{|c|}{ Little Goose } & \multicolumn{3}{|c|}{ Lower Monumental } \\
\hline & & $\begin{array}{l}\text { First } \\
\text { detection }\end{array}$ & $\begin{array}{l}\text { Previous } \\
\text { detections } \\
\text { at } 1 \text { dam }\end{array}$ & $\begin{array}{l}\text { First } \\
\text { detection }\end{array}$ & $\begin{array}{l}\text { Previous } \\
\text { detections } \\
\text { at } 1 \text { dam }\end{array}$ & $\begin{array}{l}\text { Previous } \\
\text { detections } \\
\text { at } 2 \text { dams }\end{array}$ \\
\hline 11 Apr & & & & & & \\
\hline 13 Apr & 6 & & & & & \\
\hline 14 Apr & 1 & & & & & \\
\hline 15 Apr & 3 & & & & & \\
\hline 16 Apr & 3 & & & & & \\
\hline 17 Apr & 4 & 1 & & & & \\
\hline 18 Apr & 1 & & & & & \\
\hline 19 Apr & 1 & & & & & \\
\hline 20 Apr & 2 & & & & & \\
\hline 21 Apr & 5 & & & & & \\
\hline 22 Apr & 2 & & & & & \\
\hline 23 Apr & 1 & $1(2)$ & & & & \\
\hline 25 Apr & & $1(2)$ & 2 & & & \\
\hline 26 Apr & & & 1 & & & \\
\hline 27 Apr & & & & & & \\
\hline
\end{tabular}


Appendix Table 13A. Continued.

\begin{tabular}{|c|c|c|c|c|c|c|c|}
\hline \multirow{2}{*}{\multicolumn{2}{|c|}{$\begin{array}{l}\text { Detection } \\
\text { date }\end{array}$}} & \multirow{2}{*}{$\begin{array}{c}\text { Lower Granite } \\
\text { First } \\
\text { detection }\end{array}$} & \multicolumn{2}{|c|}{ Little Goose } & \multicolumn{3}{|c|}{ Lower Monumental } \\
\hline & & & $\begin{array}{l}\text { First } \\
\text { detection }\end{array}$ & \multirow[t]{2}{*}{$\begin{array}{l}\text { Previous } \\
\text { detections } \\
\text { at } 1 \text { dam }\end{array}$} & \multirow[t]{2}{*}{$\begin{array}{l}\text { First } \\
\text { detection }\end{array}$} & \multirow{2}{*}{$\begin{array}{c}\text { Previous } \\
\text { detections } \\
\text { at } 1 \text { dam } \\
2\end{array}$} & \multirow[t]{2}{*}{$\begin{array}{l}\text { Previous } \\
\text { detections } \\
\text { at } 2 \text { dams }\end{array}$} \\
\hline 28 & Apr & 5 & 1 & & & & \\
\hline 29 & Apr & 3 & & 1 & & 1 & \\
\hline 30 & Apr & & $3(4)$ & & & 2 & \\
\hline 01 & May & 2 & 1 & 2 & 1 & & \\
\hline 02 & May & 4 & 1 & 4 & 1 & & \\
\hline 03 & May & 4 & & 3 & & & \\
\hline 04 & May & 1 & & 1 & & 1 & \\
\hline 05 & May & 1 & & & 1 & 1 & \\
\hline 06 & May & & 1 & 3 & & & \\
\hline 07 & May & $\mathbf{x}$ & $2(3)$ & 2 & & & \\
\hline 08 & May & $2(3)$ & 1 & 1 & & $i$ & \\
\hline 09 & May & $3(4)$ & & & & 1 & \\
\hline 10 & May & 2 & 1 & 2 & & & \\
\hline 11 & May & & 1 & & & & \\
\hline 12 & May & & & & & 2 & \\
\hline 13 & May & $I$ & & 1 & & & \\
\hline 17 & May & $I$ & & & & & \\
\hline 20 & May & $I$ & & & & & \\
\hline 22 & May & & & & & & \\
\hline
\end{tabular}


Appendix Table 13A. Continued.

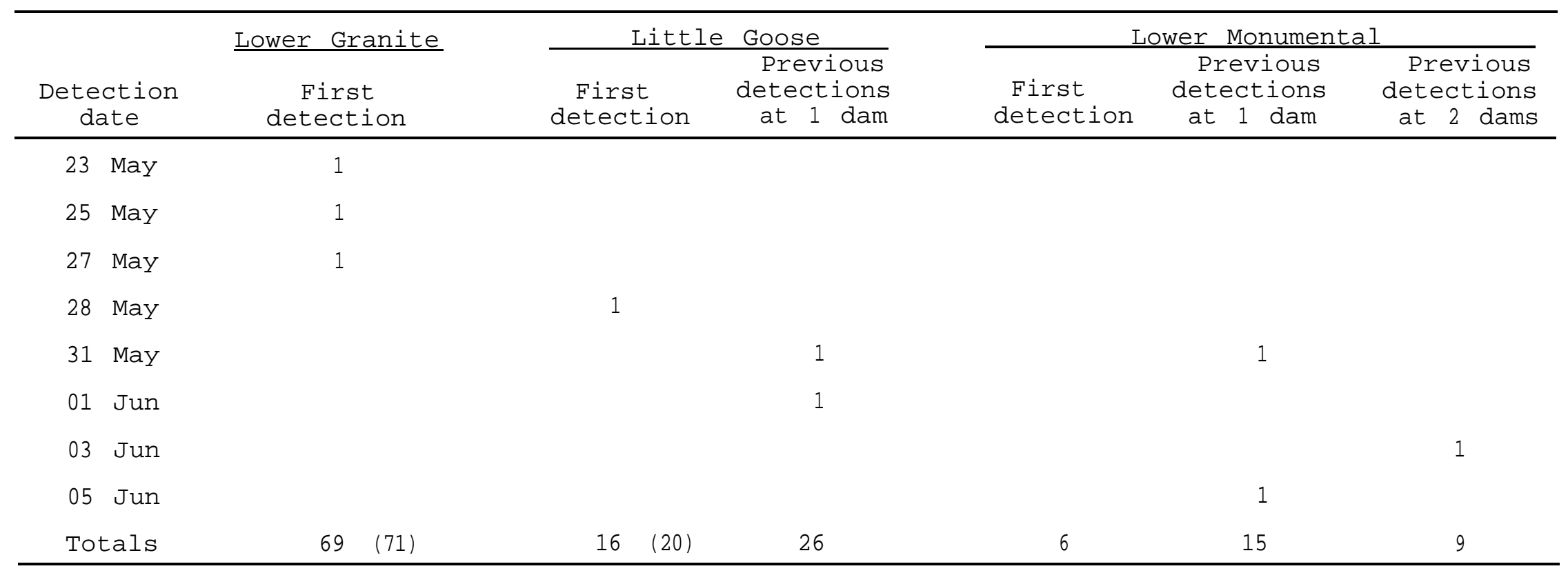


Appendix Table 13B Detections of PIT-tagged smolts by date at three Columbia River dams for wild chinook salmon from the East Fork of the Salmon River. 1995. Numbers in parentheses are first detections at the dams that have been adjusted for spill.

\begin{tabular}{|c|c|c|c|c|c|c|c|c|c|c|c|c|c|c|c|}
\hline \multirow{3}{*}{$\begin{array}{l}\text { Detection } \\
\text { daze }\end{array}$} & \multicolumn{4}{|c|}{ McNary } & \multicolumn{5}{|c|}{ 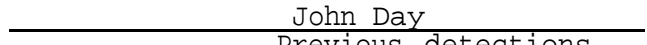 } & \multicolumn{6}{|c|}{$\frac{\text { Bonneeville }}{\text { Dreotila }}$} \\
\hline & \multirow[b]{2}{*}{ detection } & \multicolumn{3}{|c|}{ Frevious detections } & \multirow{2}{*}{$\begin{array}{c}\text { First } \\
\text { detection }\end{array}$} & \multicolumn{4}{|c|}{$\frac{\text { Previous detections }}{2}$} & \multirow{2}{*}{$\begin{array}{c}\text { First } \\
\text { detection } \\
\end{array}$} & \multirow{2}{*}{$\begin{array}{c}1 \\
\text { dam }\end{array}$} & \multirow{2}{*}{$\begin{array}{l}\frac{\frac{P r e v i d}{2}}{\text { dams }} \\
\end{array}$} & \multirow{3}{*}{$\begin{array}{l}\frac{1}{d e} \\
3 \\
\text { dams }\end{array}$} & \multirow{3}{*}{$\begin{array}{l}\text { tions } \\
\text { dams }\end{array}$} & \multirow{2}{*}{$\begin{array}{l}5 \\
\text { dams }\end{array}$} \\
\hline & & dam & darns & dams & & dam & $d^{2} m s$ & dams & $\begin{array}{c}4 \\
\text { dams }\end{array}$ & & & & & & \\
\hline 30 Apr & 1 & & & & & & & & & & & & & & \\
\hline 02 May & & 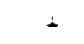 & & & & & & & & & & & & & \\
\hline 04 May & & - & & & & & & & & & & & & & \\
\hline 05 May & & & - & & & & & & & & & & & & \\
\hline 06 May & & & i & & & & & & & & & & & & \\
\hline 07 May & & & 2 & & & & & & & & & & & & \\
\hline 08 May & 1 (2) & 3 & 2 & & & & & & & & & & & & \\
\hline 09 Uа у & & 1 & 2 & & & & & & & & & & & & \\
\hline 10 May & & 2 & & & & & & & & & & & & & \\
\hline 13 May & & & - & & & & & & & & & & & & \\
\hline 14 May & $=2 i$ & & & & & & & & & & & & & & \\
\hline 16 May & & & & & & & & & & & & & & & \\
\hline 17 May & $1 \quad(2)$ & & 1 & & & & & & & & & & & & \\
\hline 24 May & & 1 & & & & & & & & & & & & & \\
\hline Totals & $4 \quad(7)$ & 10 & 11 & I & & & & & & & & & & & \\
\hline
\end{tabular}


Appendix Table 14A Detections of PIT-tagged smolts by date at three Snake River dams for wild chinook salmon from Big Creek (upper), 1995. Numbers in parentheses are first detections at the dams that have been adjusted for spill.

Tagging site: Big Creek (upper)

Release site: Big Creek (upper)

Release river kilometer(s) above Lower Granite Darn: 530 - 533
Release date: 17 Aug 1994

Number released: 757

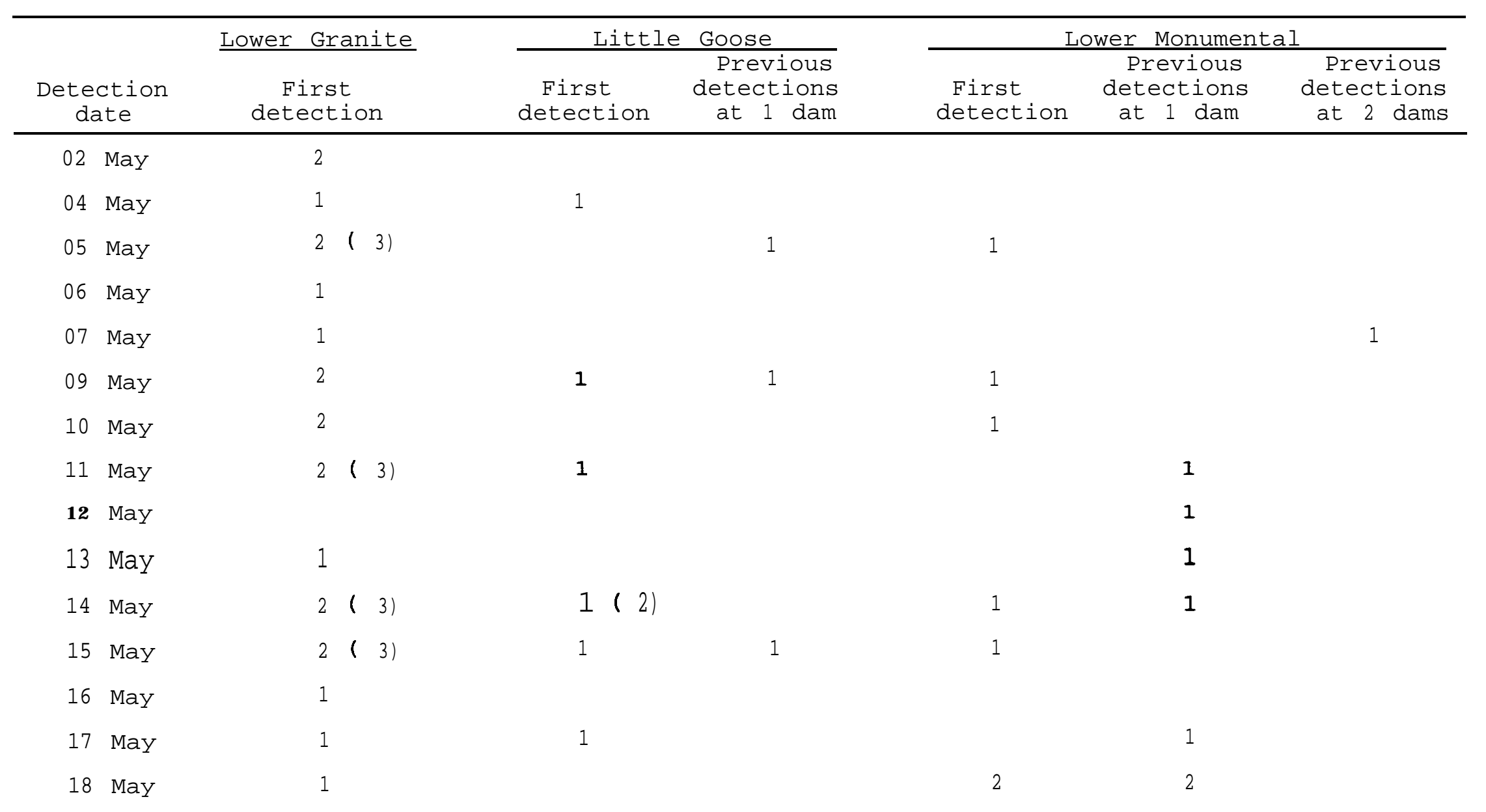


Appendix Table 14A. Continued.

\begin{tabular}{|c|c|c|c|c|c|c|c|}
\hline \multirow{2}{*}{\multicolumn{2}{|c|}{$\begin{array}{c}\text { Detection } \\
\text { date }\end{array}$}} & \multirow{2}{*}{$\begin{array}{c}\text { Lower Granite } \\
\text { First } \\
\text { detection }\end{array}$} & \multicolumn{2}{|c|}{ Little Goose } & \multicolumn{3}{|c|}{ Lower Monumental } \\
\hline & & & $\begin{array}{l}\text { First } \\
\text { detection }\end{array}$ & \multirow[t]{2}{*}{$\begin{array}{l}\text { Previous } \\
\text { detections } \\
\text { at } 1 \text { dam }\end{array}$} & $\begin{array}{l}\text { First } \\
\text { detection }\end{array}$ & $\begin{array}{l}\text { Previous } \\
\text { detections } \\
\text { at } 1 \text { dam }\end{array}$ & \multirow[t]{2}{*}{$\begin{array}{l}\text { Previous } \\
\text { detections } \\
\text { at } 2 \text { dams }\end{array}$} \\
\hline 19 & May & & $2(3)$ & & & 1 & \\
\hline 20 & May & 1 & & & & & \\
\hline 21 & May & & 1 & & & & \\
\hline 22 & May & & & 1 & & & \\
\hline 23 & May & $2(3)$ & & & & & \\
\hline 24 & May & $2(3)$ & & 1 & $2(3)$ & 1 & \\
\hline 25 & May & 1 & & & & & \\
\hline 26 & May & 1 & 2 & & & & \\
\hline 27 & May & 2 & & 1 & & & \\
\hline 28 & May & & 2 & 1 & & 1 & \\
\hline 29 & May & 3 & & 2 & & 2 & \\
\hline 30 & May & 2 & 1 & & & 1 & \\
\hline 31 & May & 1 & & & & & \\
\hline 01 & Jun & 1 & & & & & 1 \\
\hline 02 & Jun & 1 & & & & & 1 \\
\hline 03 & Jun & & 1 & 1 & & & \\
\hline 04 & Jun & & & 1 & & & \\
\hline 05 & Jun & & & 2 & & & \\
\hline 06 & Jun & & & & & 1 & \\
\hline
\end{tabular}


Appendix Table 14A. Continued.

\begin{tabular}{|c|c|c|c|c|c|c|}
\hline \multirow{2}{*}{$\begin{array}{l}\text { Detection } \\
\text { date }\end{array}$} & \multirow{3}{*}{ 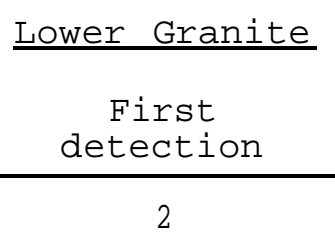 } & \multicolumn{2}{|c|}{ Little Goose } & \multicolumn{3}{|c|}{ Lower Monumental } \\
\hline & & $\begin{array}{c}\text { First } \\
\text { detection }\end{array}$ & $\begin{array}{c}\text { Previous } \\
\text { detections } \\
\text { at } 1 \text { dam }\end{array}$ & $\begin{array}{l}\text { First } \\
\text { detection }\end{array}$ & $\begin{array}{l}\text { Previous } \\
\text { detections } \\
\text { at } 1 \text { dam }\end{array}$ & $\begin{array}{l}\text { Previous } \\
\text { detections } \\
\text { at } 2 \text { dams }\end{array}$ \\
\hline 07 Jun & & & & 1 & & \\
\hline 08 Jun & 1 & 1 & & 1 & & \\
\hline 09 Jun & 2 & & 1 & & & \\
\hline 10 Jun & & 2 & & 1 & 1 & \\
\hline 11 Jun & 1 & & 1 & & & \\
\hline 12 Jun & & 1 & & 1 & 1 & \\
\hline 13 Jun & & & & & 1 & \\
\hline 14 Jun & & & & & & 1 \\
\hline 15 Jun & & & & & & 1 \\
\hline 17 Jun & & 1 & & & & \\
\hline 19 Jun & 1 & & & 1 & & \\
\hline 20 Jun & 1 & & & & & \\
\hline 21 Jun & 1 & & & & & \\
\hline 22 Jun & 1 & & 1 & & & \\
\hline 24 Jun & & & & & & 1 \\
\hline 26 Jun & 1 & & & & & \\
\hline 27 Jun & & & 2 & & & \\
\hline 29 Jun & & & & & & \\
\hline $01 \mathrm{Jul}$ & & & & & & \\
\hline
\end{tabular}


Appendix Table 14A. Continued.

\begin{tabular}{|c|c|c|c|c|c|c|}
\hline \multirow[b]{2}{*}{$\begin{array}{c}\text { Detection } \\
\text { date }\end{array}$} & \multirow{2}{*}{$\begin{array}{c}\text { Lower Granite } \\
\text { First } \\
\text { detection }\end{array}$} & \multicolumn{2}{|c|}{ Little Goose } & \multicolumn{3}{|c|}{ Lower Monumental } \\
\hline & & $\begin{array}{c}\text { First } \\
\text { detection }\end{array}$ & $\begin{array}{l}\text { Previous } \\
\text { detections } \\
\text { at } \mathbf{1} \text { dam }\end{array}$ & $\begin{array}{l}\text { First } \\
\text { detection }\end{array}$ & $\begin{array}{l}\text { Previous } \\
\text { detections } \\
\text { at } \mathbf{1} \text { dam }\end{array}$ & $\begin{array}{l}\text { Previous } \\
\text { detections } \\
\text { at } 2 \text { dams }\end{array}$ \\
\hline $03 \mathrm{Jul}$ & & & 1 & & & \\
\hline $07 \mathrm{Jul}$ & & & & & & 1 \\
\hline $11 \mathrm{Jul}$ & & & 1 & & & \\
\hline $21 \mathrm{Jul}$ & & 1 & & & & \\
\hline 27 Jul & & & & & & 1 \\
\hline 28 Aug & & 1 & & & & \\
\hline Totals & $50 \quad(56)$ & $23 \quad(25)$ & 21 & 16 (17) & 20 & 11 \\
\hline
\end{tabular}


Appendix Table 14B. Detections of PIT-tagged smolts by date at three Columbia River dams for wild chinook salmon from Big Creek (upper). 1995. Numbers in parentheses are first detections at the dams that have been adjusted for spill.

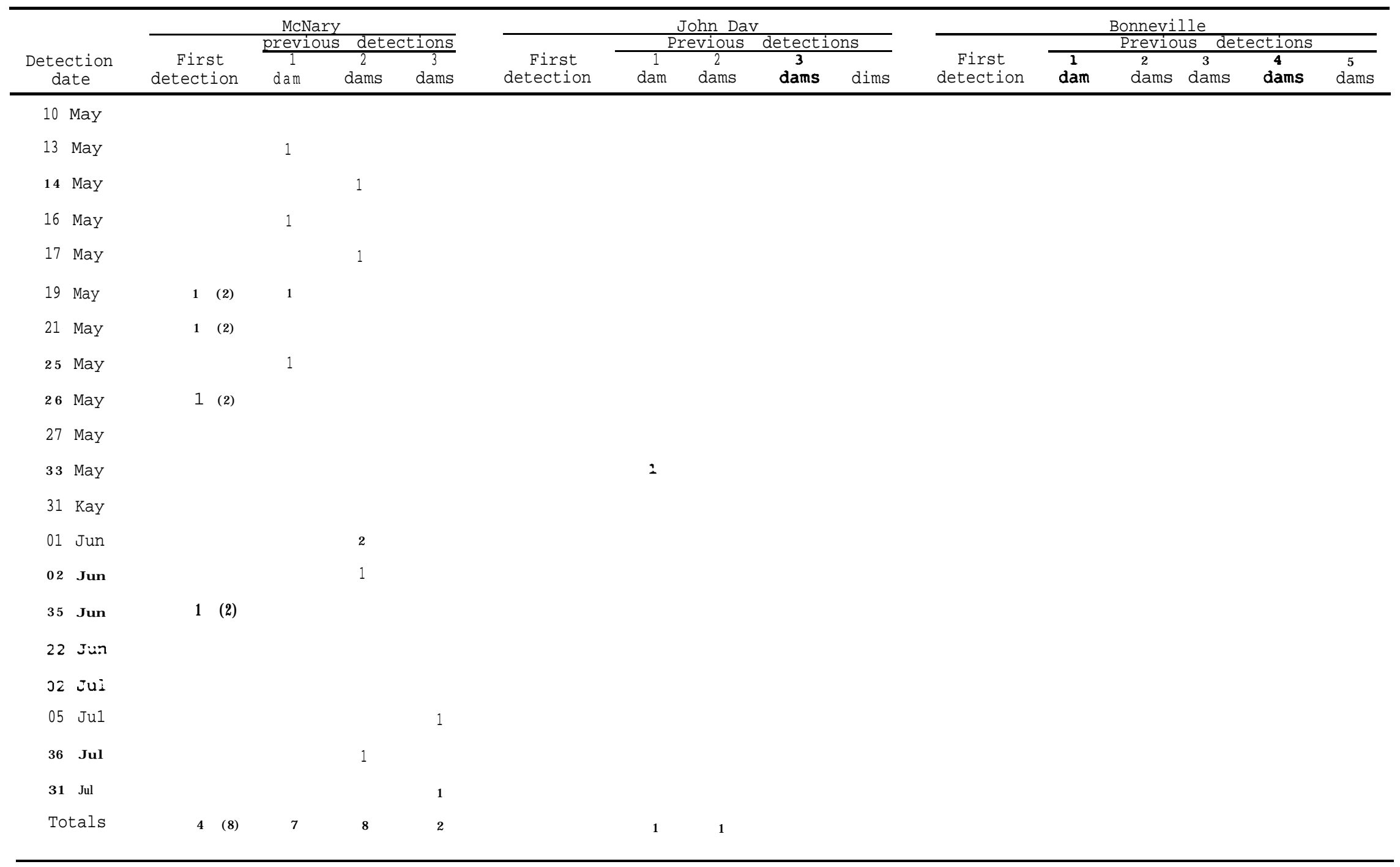


Appendix Table 15A. Detections of PIT-tagged smolts by date at three Snake River dams for wild chinook salmon from the South Fork of the Salmon River. 1995. Numbers in parentheses are first detections at the dams that have been adjusted for spill.

Tagging site: S. F. Salmon River

Release site: S. F. Salmon River

Release date: 16 - 18 Aug 1994

Release river kilometer(s) above Lower Granite Dam: 357 - 468

Number released: 1.571

\begin{tabular}{|c|c|c|c|c|c|c|}
\hline \multirow{2}{*}{$\begin{array}{c}\text { Detection } \\
\text { date }\end{array}$} & \multirow{3}{*}{ 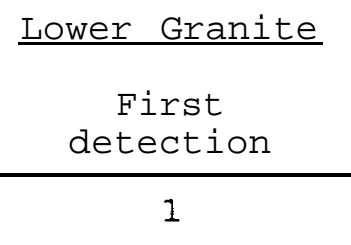 } & \multicolumn{2}{|c|}{ Little Goose } & \multicolumn{3}{|c|}{ Lower Monumental } \\
\hline & & \multirow[t]{2}{*}{$\begin{array}{l}\text { First } \\
\text { detection }\end{array}$} & \multirow[t]{2}{*}{$\begin{array}{l}\text { Previous } \\
\text { detections } \\
\text { at } 1 \text { dam }\end{array}$} & $\begin{array}{l}\text { First } \\
\text { detection }\end{array}$ & $\begin{array}{l}\text { Previous } \\
\text { detections } \\
\text { at } 1 \text { dam }\end{array}$ & \multirow[t]{2}{*}{$\begin{array}{l}\text { Previous } \\
\text { detections } \\
\text { at } 2 \text { dams }\end{array}$} \\
\hline 13 Apr & & & & & & \\
\hline 14 Apr & 2 & & & & & \\
\hline 15 Apr & 2 & & & & & \\
\hline 16 Apr & $\Sigma$ & & & & & \\
\hline 17 Apr & & 2 & & & & \\
\hline 18 Apr & $\perp$ & & & & & \\
\hline 19 Apr & 1 & & & & & \\
\hline 20 Apr & 1 & & 1 & & & \\
\hline 21 Apr & 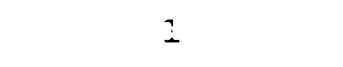 & & 1 & & & \\
\hline 22 Apr & & & & & & 1 \\
\hline 24 Apr & 1 & & & & & \\
\hline 25 Apr & & & & & & 1 \\
\hline 27 Apr & I & & & & & \\
\hline 28 Apr & 1 & & & & & \\
\hline 29 Apr & 2 & & & & & \\
\hline
\end{tabular}


Appendix Table 15A. Continued.

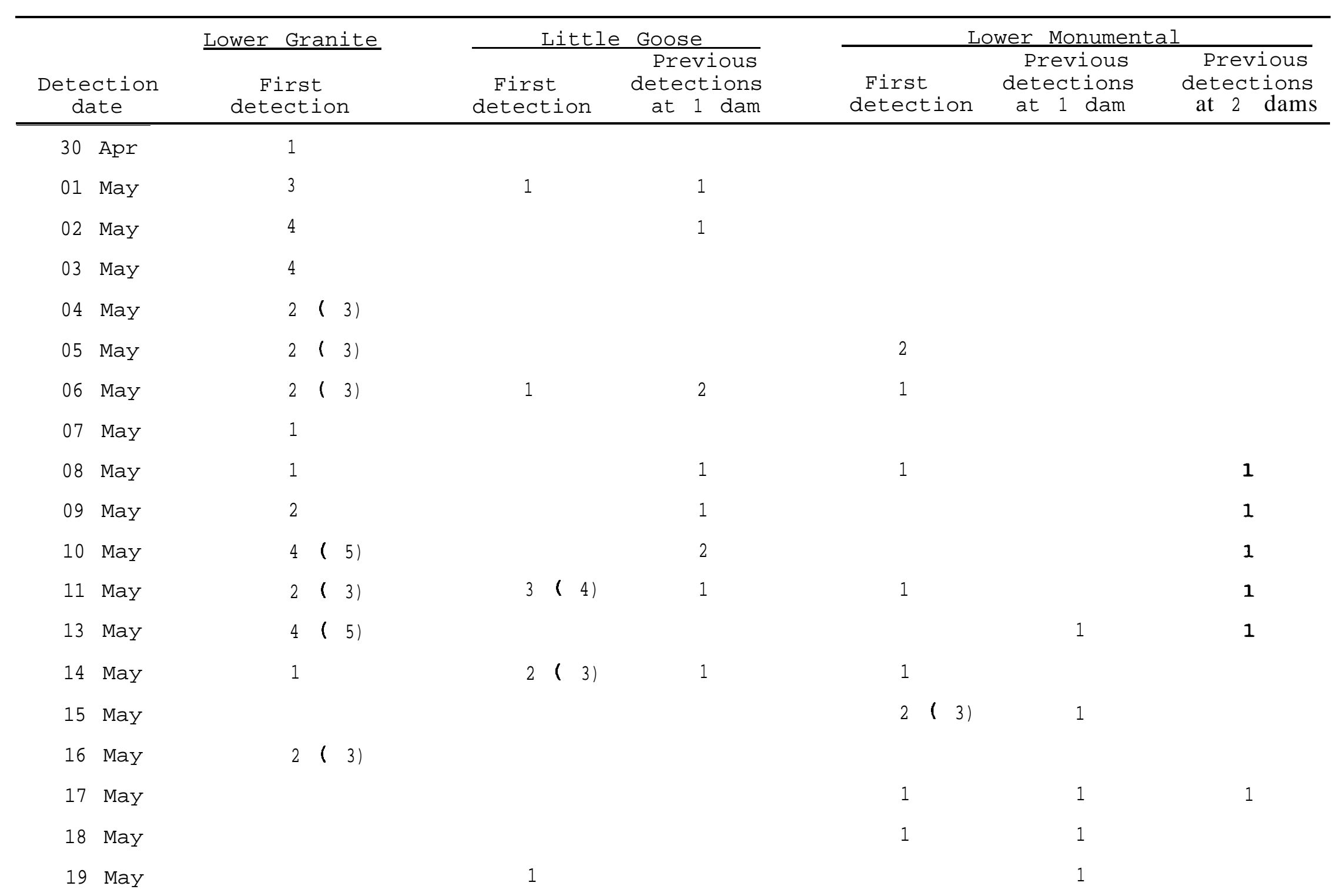


Appendix Table 15A. Continued.

\begin{tabular}{|c|c|c|c|c|c|c|c|}
\hline \multirow{2}{*}{\multicolumn{2}{|c|}{$\begin{array}{l}\text { Detection } \\
\text { date }\end{array}$}} & \multirow{3}{*}{$\begin{array}{c}\text { Lower Granite } \\
\begin{array}{c}\text { First } \\
\text { detection }\end{array} \\
1\end{array}$} & \multicolumn{2}{|c|}{ Little Goose } & \multicolumn{3}{|c|}{ Lower Monumental } \\
\hline & & & $\begin{array}{c}\text { First } \\
\text { detection }\end{array}$ & $\begin{array}{l}\text { Previous } \\
\text { detections } \\
\text { at } 1 \text { dam }\end{array}$ & \multirow{2}{*}{$\frac{\begin{array}{c}\text { First } \\
\text { detection }\end{array}}{1}$} & \multirow[t]{2}{*}{$\begin{array}{l}\text { Previous } \\
\text { detections } \\
\text { at } 1 \text { dam }\end{array}$} & \multirow[t]{2}{*}{$\begin{array}{l}\text { Previous } \\
\text { detections } \\
\text { at } 2 \text { dams }\end{array}$} \\
\hline 20 & May & & $2(3)$ & 1 & & & \\
\hline 21 & May & 1 & & & & & \\
\hline 22 & May & & & & 1 & 1 & \\
\hline 23 & May & 1 & & & & 3 & \\
\hline 24 & May & 1 & 1 & & & & \\
\hline 25 & May & & 1 & & & & \\
\hline 26 & May & & & & & & \\
\hline 27 & May & 2 & & & & & \\
\hline 28 & May & 2 & & & & & 1 \\
\hline 29 & May & 1 & & & & & \\
\hline 30 & May & 2 & & 1 & & & \\
\hline 01 & Jun & 3 & & & & & 1 \\
\hline 02 & Jun & & 1 & & 1 & & \\
\hline 03 & Jun & 1 & & & & & \\
\hline 04 & Jun & 2 & & & & & \\
\hline 05 & Jun & 1 & & & 1 & & \\
\hline 06 & Jun & 2 & & & $\beth$ & & \\
\hline 07 & Jun & & $1(2)$ & & & & \\
\hline 08 & Jun & & & & & & \\
\hline
\end{tabular}


Appendix Table I5A. Continued.

\begin{tabular}{|c|c|c|c|c|c|c|}
\hline \multirow[b]{2}{*}{$\begin{array}{c}\text { Detection } \\
\text { date }\end{array}$} & \multirow{2}{*}{$\begin{array}{c}\text { Lower Granite } \\
\text { First } \\
\text { detection }\end{array}$} & \multicolumn{2}{|c|}{ Little Goose } & \multicolumn{3}{|c|}{ Lower Monumental } \\
\hline & & $\begin{array}{c}\text { First } \\
\text { detection }\end{array}$ & $\begin{array}{r}\text { Previous } \\
\text { detections } \\
\text { at } 1 \text { dam }\end{array}$ & $\begin{array}{l}\text { First } \\
\text { detection }\end{array}$ & $\begin{array}{l}\text { Previous } \\
\text { detections } \\
\text { at } 1 \text { dam }\end{array}$ & $\begin{array}{l}\text { Previous } \\
\text { detections } \\
\text { at } 2 \text { dams }\end{array}$ \\
\hline 09 Jun & 1 & & & & 1 & \\
\hline $10 \mathrm{Jun}$ & $2(5)$ & 1 & & & & \\
\hline 11 Jun & 1 & 1 & & & 1 & 1 \\
\hline 12 Jun & & & & 1 & & \\
\hline 13 Jun & & 1 & & & 1 & \\
\hline 14 Jun & & & & 2 & 1 & \\
\hline 17 Jun & & & & & 1 & \\
\hline 18 Jun & & & & & $i$ & \\
\hline 19 Jun & & & 1 & & & \\
\hline 20 Jun & & & 1 & & 1 & \\
\hline 22 Jun & 1 & & & & & \\
\hline 24 Jun & & 1 & & & & \\
\hline 25 Jun & & 1 & & & & \\
\hline 27 Jun & & & & & 1 & \\
\hline 28 Jun & & & & & 1 & \\
\hline 29 Jun & & & & & & \\
\hline $02 \mathrm{Jul}$ & & & & & & \\
\hline 06 Jul & 1 & & & & & \\
\hline $08 \mathrm{Jul}$ & & & & & & \\
\hline
\end{tabular}


Appendix Table 15A. Continued.

\begin{tabular}{|c|c|c|c|c|c|c|}
\hline \multirow[b]{2}{*}{$\begin{array}{c}\text { Detection } \\
\text { date }\end{array}$} & \multirow{2}{*}{$\begin{array}{c}\text { Lower Granite } \\
\text { First } \\
\text { detection }\end{array}$} & \multicolumn{2}{|c|}{ Little Goose } & \multicolumn{3}{|c|}{ Lower Monumental } \\
\hline & & $\begin{array}{c}\text { First } \\
\text { detection }\end{array}$ & $\begin{array}{r}\text { Previous } \\
\text { detections } \\
\text { at } 1 \text { dam }\end{array}$ & $\begin{array}{l}\text { First } \\
\text { detection }\end{array}$ & $\begin{array}{l}\text { Previous } \\
\text { detections } \\
\text { at } 1 \text { dam }\end{array}$ & $\begin{array}{l}\text { Previous } \\
\text { detections } \\
\text { at } 2 \text { dams }\end{array}$ \\
\hline $10 \mathrm{Jul}$ & & & 2 & & 1 & \\
\hline $13 \mathrm{Jul}$ & 1 & & & & & \\
\hline $22 \mathrm{Jul}$ & & & & & & \\
\hline $26 \mathrm{Jul}$ & & & & & 1 & \\
\hline Totals & $78 \quad(88)$ & $(27)$ & 23 & $(20)$ & 30 & 12 \\
\hline
\end{tabular}


Appendix Table 15B Detections of PIT-tagged smolts by date at three Columbia River dams for wild chinook salmon from the South Fork of the Salmon River, 1995. Numbers in parentheses are first detections at the dams that have been adjusted for spill.

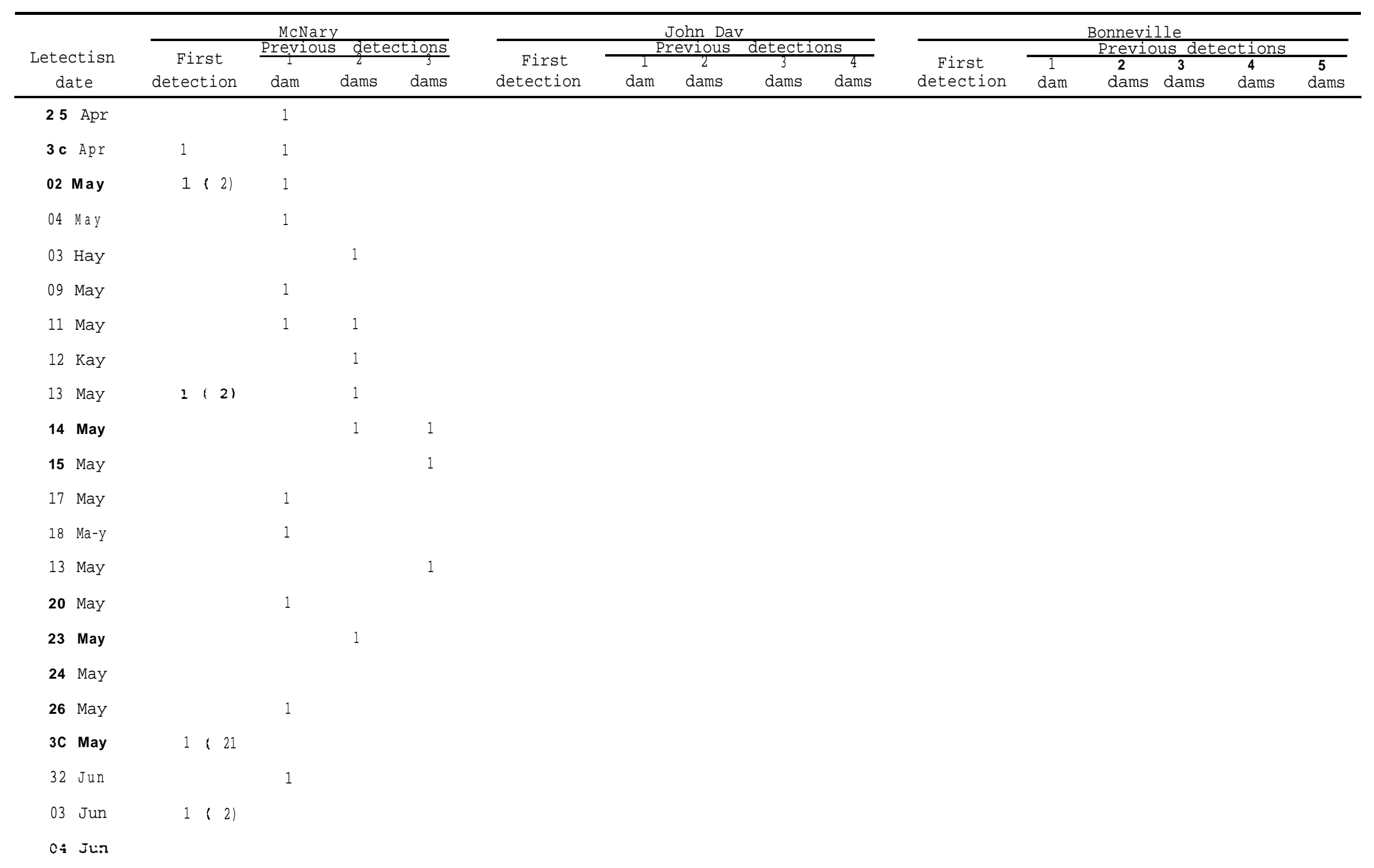


Appendix Table 1 5B. Continued.

\begin{tabular}{|c|c|c|c|c|c|c|c|c|c|c|c|c|c|c|c|c|}
\hline \multirow[b]{3}{*}{$\begin{array}{l}\text { Detection. } \\
\text { dare }\end{array}$} & \multicolumn{5}{|c|}{ MeNary } & \multicolumn{5}{|c|}{ John Day } & \multicolumn{6}{|c|}{ Bonneville } \\
\hline & \multirow{2}{*}{\multicolumn{2}{|c|}{$\begin{array}{c}\text { First } \\
\text { detection }\end{array}$}} & \multicolumn{3}{|c|}{ Previous detections } & \multirow[b]{2}{*}{ detectrsiton } & \multicolumn{4}{|c|}{ Previous detections } & \multirow[b]{2}{*}{$\begin{array}{l}\text { First } \\
\text { detection }\end{array}$} & \multicolumn{5}{|c|}{ Previous detections } \\
\hline & & & $\begin{array}{c}1 \\
\text { dam }\end{array}$ & dams & $\overline{d a^{3} m s}$ & & dam & $d a^{2} m s$ & $\begin{array}{c}3 \\
\text { dams }\end{array}$ & $\begin{array}{c}4 \\
\text { dams }\end{array}$ & & $\begin{array}{c}1 \\
\text { dam }\end{array}$ & $\begin{array}{c}2 \\
\text { dams }\end{array}$ & $\begin{array}{c}3 \\
\text { dams }\end{array}$ & $\begin{array}{c}4 \\
\text { dans }\end{array}$ & $\begin{array}{c}5 \\
\text { dams }\end{array}$ \\
\hline \multicolumn{17}{|l|}{ C5 Jin } \\
\hline $06 \mathrm{~J}$ un & 1 & 2 & & & & & & & & & & & & & & \\
\hline \multicolumn{17}{|l|}{19 Jun } \\
\hline $13 \mathrm{Jul}$ & & & & 1 & & & & & & & & & & & & \\
\hline 01 Aug & & & & 1 & & & & & & & & & & & & \\
\hline Totals & 6 & 11 & 13 & 8 & 4 & & & & 1 & & & & & & & \\
\hline
\end{tabular}


Appendix Table 16A Detections of PIT-tagged smolts by date at three Snake River dams for wild chinook salmon from Big Creek (lower). 1995. Numbers in parentheses are first detections at the dams that have been adjusted for spill.

Tagging site: Big Creek (lower)

Release date: 21 Aug 1994

Release site: Big Creek (Iower)

Release river kilometer(s) above Lower Granite Dam: 488 - 491

Number released: 727

\begin{tabular}{|c|c|c|c|c|c|c|}
\hline \multirow[b]{2}{*}{$\begin{array}{c}\text { Detection } \\
\text { date }\end{array}$} & \multirow{2}{*}{$\begin{array}{c}\text { Lower Granite } \\
\text { First } \\
\text { detection }\end{array}$} & \multicolumn{2}{|c|}{ Little Goose } & \multicolumn{3}{|c|}{ Lower Monumental } \\
\hline & & $\begin{array}{c}\text { First } \\
\text { detection }\end{array}$ & $\begin{array}{l}\text { Previous } \\
\text { detections } \\
\text { at } 1 \text { dam }\end{array}$ & $\begin{array}{l}\text { First } \\
\text { detection }\end{array}$ & $\begin{array}{l}\text { Previous } \\
\text { detections } \\
\text { at } 1 \text { dam }\end{array}$ & $\begin{array}{l}\text { Previous } \\
\text { detections } \\
\text { at } 2 \text { dams }\end{array}$ \\
\hline 11 Apr & 1 & & & & & \\
\hline 12 Apr & 1 & & & & & \\
\hline 13 Apr & 2 & & & & & \\
\hline 14 Apr & 1 & & & & & \\
\hline 15 Apr & & & & & & \\
\hline 16 Apr & 1 & & & & & \\
\hline 17 Apr & 5 & & 1 & & 1 & \\
\hline 18 Apr & 1 & & & & & \\
\hline 19 Apr & 1 & & & & & \\
\hline 20 Apr & 6 & & & & 1 & \\
\hline 21 Apr & 5 & & & & & \\
\hline 23 Apr & 2 & $1(2)$ & & & & \\
\hline 24 Apr & 1 & & & & & \\
\hline 25 Apr & 4 & & & & & \\
\hline 26 Apr & 9 & & & & 1 & \\
\hline
\end{tabular}


Appendix Table 16A. Continued.

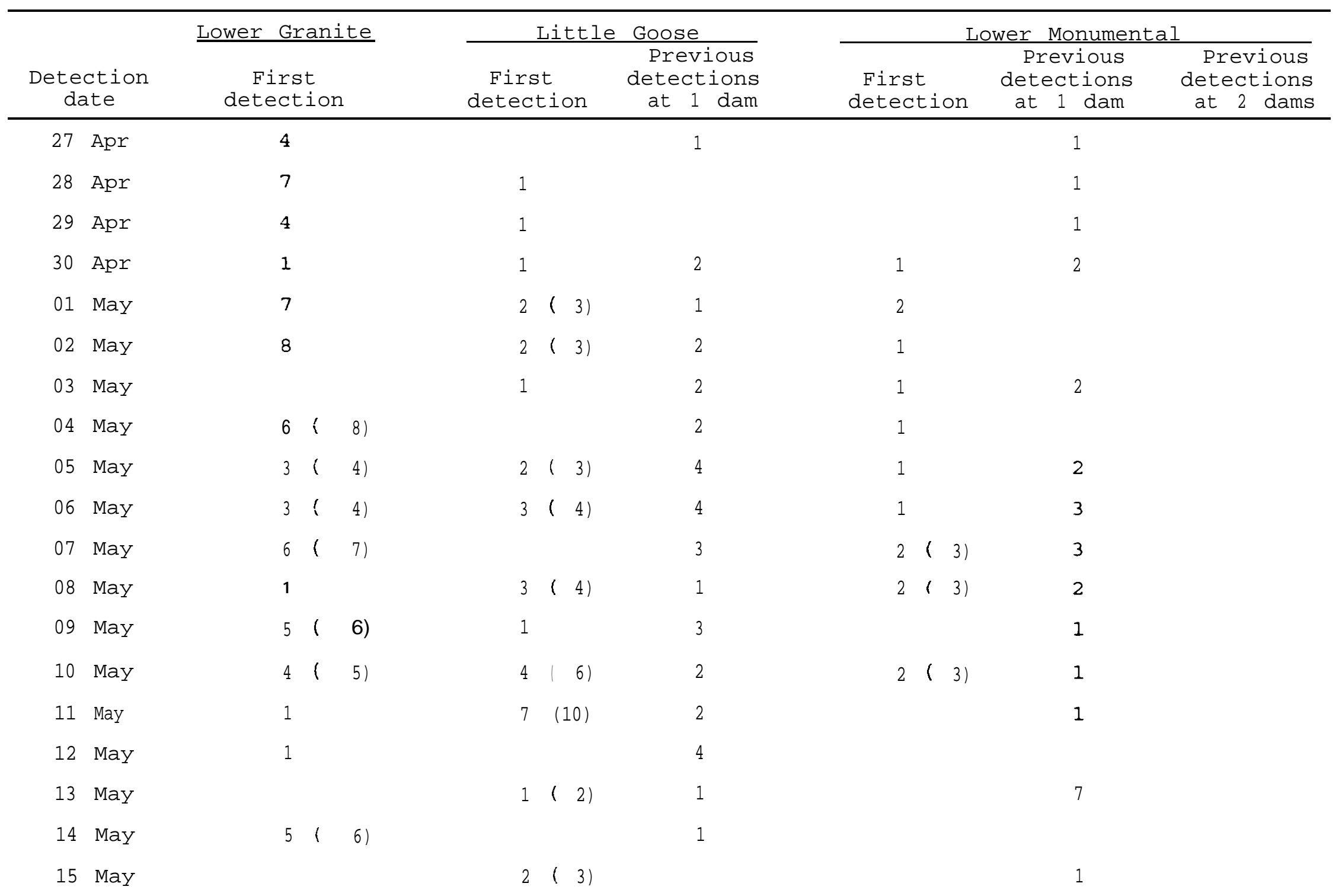


Appendix Table 16A. Continued.

\begin{tabular}{|c|c|c|c|c|c|c|c|}
\hline \multirow[b]{2}{*}{$\begin{array}{c}\text { Detection } \\
\text { date }\end{array}$} & \multirow{2}{*}{$\begin{array}{c}\text { Lower Granite } \\
\text { First } \\
\text { detection }\end{array}$} & \multicolumn{2}{|c|}{ Little Goose } & & \multicolumn{3}{|c|}{ Lower Monumental } \\
\hline & & $\begin{array}{c}\text { First } \\
\text { detection }\end{array}$ & $\begin{array}{r}\text { Previous } \\
\text { detections } \\
\text { at } 1 \text { dam }\end{array}$ & & $\begin{array}{l}\text { First } \\
\text { detection }\end{array}$ & $\begin{array}{l}\text { Previous } \\
\text { detections } \\
\text { at } 1 \text { dam }\end{array}$ & $\begin{array}{l}\text { Previous } \\
\text { detections } \\
\text { at } 2 \text { dams }\end{array}$ \\
\hline 16 May & & & & & 1 & & 1 \\
\hline 17 May & & & 1 & & $3(4)$ & & \\
\hline 18 May & 1 & & & & & 3 & 1 \\
\hline 19 May & 1 & & 1 & & & & \\
\hline 20 May & $2(3)$ & & & 1 & & & \\
\hline 21 May & & & & & & 1 & \\
\hline 22 May & & & & & & 2 & 1 \\
\hline 23 May & & & & & & 2 & \\
\hline 24 May & 1 & & 1 & & & & \\
\hline 25 May & & 2 & 1 & & & & \\
\hline 26 May & & & & & & & \\
\hline 27 May & & & 1 & & & & \\
\hline 31 May & & & & & & & \\
\hline 02 Jun & & & & & & 1 & \\
\hline 05 Jun & 1 & & & & & & \\
\hline 10 Jun & & & & & & 1 & \\
\hline Totals & $(121)$ & (50) & 42 & & $22 \quad(26)$ & 41 & 21 \\
\hline
\end{tabular}


Appendix Table 16B. Detections of PIT-tagged smolts by date at three Columbia River dams for wild chinook salmon from Big Creek (lower), 1995. Numbers in parentheses are first detections at the dams that have been adjusted for spill.

\begin{tabular}{|c|c|c|c|c|c|c|c|c|c|c|c|c|c|c|c|}
\hline \multirow[b]{3}{*}{$\begin{array}{c}\text { Detection } \\
\text { dare }\end{array}$} & \multirow{2}{*}{\multicolumn{4}{|c|}{ McNarv }} & \multirow{2}{*}{\multicolumn{5}{|c|}{ John Day }} & \multirow{2}{*}{\multicolumn{6}{|c|}{ Bonneville }} \\
\hline & & & & & & & & & & & & & & tions & \\
\hline & $\begin{array}{c}\text { First } \\
\text { detection }\end{array}$ & $\begin{array}{c}1 \\
\text { darn }\end{array}$ & $\begin{array}{c}2 \\
\text { dams }\end{array}$ & $\begin{array}{c}3 \\
\text { dams }\end{array}$ & $\begin{array}{c}\text { First } \\
\text { detectcion }\end{array}$ & dam & $\begin{array}{c}2 \\
\text { dams }\end{array}$ & $\begin{array}{c}3 \\
\text { dams }\end{array}$ & $\begin{array}{c}4 \\
\text { dams }\end{array}$ & $\begin{array}{c}\text { First } \\
\text { detection }\end{array}$ & $\begin{array}{c}1 \\
\text { dam }\end{array}$ & $\begin{array}{c}2 \\
\text { dams }\end{array}$ & $\begin{array}{c}3 \\
\text { dams }\end{array}$ & $\begin{array}{c}4 \\
\text { dams }\end{array}$ & $\begin{array}{c}5 \\
\text { dams }\end{array}$ \\
\hline
\end{tabular}

\begin{tabular}{|c|c|c|c|c|}
\hline $30 \mathrm{Apr}$ & 1 & $i$ & & \\
\hline 01 May & & $i$ & & \\
\hline 02 May & & & & \\
\hline 03 May & $1: 2)$ & & & \\
\hline 04 May & $1: 2^{\prime}$ & 1 & 1 & \\
\hline 05 May & & & 1 & \\
\hline 06 May & & 2 & & \\
\hline 07 May & & & 1 & \\
\hline 08 May & & & & \\
\hline 09 May & $1: 2$ & & 1 & \\
\hline 10 Hay & & & & 2 \\
\hline 11 Uay & & 3 & $i$ & \\
\hline ii May & & 1 & & 1 \\
\hline 14 Kay & & 2 & 2 & \\
\hline 16 May & $2 \quad 4 !$ & 1 & & \\
\hline 17 Kay & I $\quad 2:$ & 1 & 1 & \\
\hline 18 Kay & & & & \\
\hline 19May & & & & \\
\hline 20 May & & 1 & & \\
\hline 21 May & & & 2 & \\
\hline 22 May & & 1 & & \\
\hline
\end{tabular}


Appendix Table 16B. Continued.

\begin{tabular}{|c|c|c|c|c|c|c|c|c|c|c|c|c|c|c|c|}
\hline \multirow[b]{3}{*}{$\begin{array}{c}\text { Detection } \\
\text { date }\end{array}$} & \multicolumn{4}{|c|}{ McNary } & \multicolumn{5}{|c|}{ John Day } & \multicolumn{6}{|c|}{ Bonneville } \\
\hline & \multirow[b]{2}{*}{$\begin{array}{c}\text { First } \\
\text { detection }\end{array}$} & \multirow{2}{*}{$\begin{array}{c}\text { Previ } \\
\begin{array}{c}1 \\
\text { dam }\end{array}\end{array}$} & \multirow{2}{*}{$\begin{array}{c}\text { det } \\
2 \\
\text { dams }\end{array}$} & \multirow{2}{*}{$\frac{\frac{\text { tions }}{3}}{\text { dams }}$} & \multirow[b]{2}{*}{$\begin{array}{c}\text { First } \\
\text { detection }\end{array}$} & \multicolumn{4}{|c|}{ Previous detections } & \multirow[b]{2}{*}{$\begin{array}{c}\text { First } \\
\text { detection }\end{array}$} & \multicolumn{5}{|c|}{ Previous detections } \\
\hline & & & & & & $\begin{array}{c}1 \\
\text { dam }\end{array}$ & $\begin{array}{c}2 \\
\text { dams }\end{array}$ & $\begin{array}{c}3 \\
\text { dams }\end{array}$ & $\begin{array}{c}\frac{4}{\text { dams }} \\
\end{array}$ & & $\begin{array}{c}1 \\
\text { dam }\end{array}$ & $\begin{array}{c}2 \\
\text { dams }\end{array}$ & $\begin{array}{c}3 \\
\text { dams }\end{array}$ & $\begin{array}{c}4 \\
\text { dams }\end{array}$ & $\begin{array}{c}5 \\
\text { dams }\end{array}$ \\
\hline 23 May & & & & 1 & & & & & & & & & & & \\
\hline 28 May & & & & 1 & & & & & & & & & & & \\
\hline Totals & $7 \quad(13)$ & 20 & 10 & 8 & & & 1 & & & & & & 1 & & \\
\hline
\end{tabular}


Appendix Table 17A. Detections of PIT-tagged smolts by date at three Snake River dams for wild chinook salmon from Rush Creek, 1995. Numbers in parentheses are first detections at the dams that have been adjusted for spill.

Tagging site: Rush Creek

Release date: 21 Aug 1994

Release site: Rush Creek

Number released: 15

Release river kilometer(s) above Lower Granite Dam: 490

\begin{tabular}{|c|c|c|c|c|c|c|}
\hline \multirow[b]{2}{*}{$\begin{array}{c}\text { Detection } \\
\text { date }\end{array}$} & \multirow{2}{*}{$\begin{array}{c}\text { Lower Granite } \\
\text { First } \\
\text { detection }\end{array}$} & \multicolumn{2}{|c|}{ Little Goose } & \multicolumn{3}{|c|}{ Lower Monumental } \\
\hline & & $\begin{array}{l}\text { First } \\
\text { detection }\end{array}$ & $\begin{array}{c}\text { Previous } \\
\text { detections } \\
\text { at } 1 \text { dam. }\end{array}$ & $\begin{array}{l}\text { First } \\
\text { detection }\end{array}$ & $\begin{array}{c}\text { Previous } \\
\text { detections } \\
\text { at } 1 \text { dam }\end{array}$ & $\begin{array}{r}\text { Previous } \\
\text { detections } \\
\text { at } 2 \text { dams }\end{array}$ \\
\hline 29 Apr & 1 & & & & & \\
\hline 05 May & & & & & 1 & \\
\hline 07 May & & & & & & \\
\hline 09 May & 1 & & & & & \\
\hline 17 May & & & & & 1 & \\
\hline Totals & 2 & 1 & & 1 & 2 & \\
\hline
\end{tabular}


Appendix Table 17B. Detections of PIT-tagged smolts by date at three Columbia River dams for wild chinook salmon from Rush Creek, 1995. Numbers in parentheses are first detections at the dams that have been adjusted for spill.

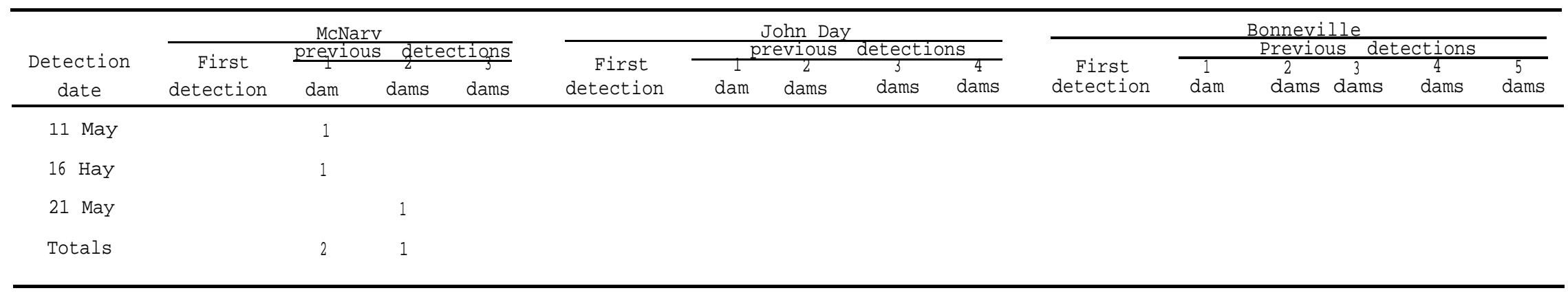


Appendix Table 18A. Detections of PIT-tagged smolts by date at three Snake River dams for wild chinook salmon from the West Fork of Chamberlain Creek, 1995. Numbers in parentheses are first detections at the dams that have been adjusted for spill.

Tagging site: W. F. Chamberlain Creek

Release site: W. F. Chamberlain Creek

Release river kilometer(s) above Lower Granite Dam: 437 - 438

Release date: 21 Aug 1994

Number released: 917

\begin{tabular}{|c|c|c|c|c|c|c|}
\hline \multirow{2}{*}{$\begin{array}{c}\text { Detection } \\
\text { date }\end{array}$} & \multirow{3}{*}{$\begin{array}{c}\text { Lower Granite } \\
\begin{array}{c}\text { First } \\
\text { detection }\end{array} \\
2\end{array}$} & \multicolumn{2}{|c|}{ Little Goose } & \multicolumn{3}{|c|}{ Lower Monumental } \\
\hline & & \multirow[t]{2}{*}{$\begin{array}{c}\text { First } \\
\text { detection }\end{array}$} & \multirow[t]{2}{*}{$\begin{array}{l}\text { Previous } \\
\text { detections } \\
\text { at } 1 \text { dam }\end{array}$} & $\begin{array}{l}\text { First } \\
\text { detection }\end{array}$ & $\begin{array}{l}\text { Previous } \\
\text { detections } \\
\text { at } 1 \text { dam }\end{array}$ & \multirow[t]{2}{*}{$\begin{array}{l}\text { Previous } \\
\text { detections } \\
\text { at } 2 \text { dams }\end{array}$} \\
\hline 12 Apr & & & & & & \\
\hline 13 Apr & 1 & & & & & \\
\hline 14 Apr & 2 & & & & & \\
\hline 15 Apr & 1 & 1 & & & & \\
\hline 16 Apr & 2 & & & & & \\
\hline 17 Apr & & & 1 & & & \\
\hline 19 Apr & 2 & & & & & \\
\hline 20 Apr & & & & & 1 & \\
\hline 22 Apr & & & 1 & & & 1 \\
\hline 23 Apr & & & & & 1 & \\
\hline 27 Apr & 1 & & & & & \\
\hline 29 Apr & & & & & & \\
\hline 01 May & 1 & & & & & \\
\hline 02 May & 2 & & 1 & & & \\
\hline 03 May & 1 & & & & & \\
\hline
\end{tabular}


Appendix Table 18A. Continued

\begin{tabular}{|c|c|c|c|c|c|c|c|}
\hline \multirow{2}{*}{\multicolumn{2}{|c|}{$\begin{array}{l}\text { Detection } \\
\text { date }\end{array}$}} & \multirow{2}{*}{$\begin{array}{c}\text { Lower Granite } \\
\text { First } \\
\text { detection }\end{array}$} & \multicolumn{2}{|c|}{ Little Goose } & \multicolumn{3}{|c|}{ Lower Monumental } \\
\hline & & & $\begin{array}{l}\text { First } \\
\text { detection }\end{array}$ & \multirow[t]{2}{*}{$\begin{array}{l}\text { Previous } \\
\text { detections } \\
\text { at } 1 \text { dam }\end{array}$} & $\begin{array}{l}\text { First } \\
\text { detection }\end{array}$ & \multirow[t]{2}{*}{$\begin{array}{l}\text { Previous } \\
\text { detections } \\
\text { at } 1 \text { dam }\end{array}$} & \multirow[t]{2}{*}{$\begin{array}{l}\text { Previous } \\
\text { detections } \\
\text { at } 2 \text { dams }\end{array}$} \\
\hline 04 & May & $3(4)$ & & & & & \\
\hline & May & & & & 1 & & \\
\hline 06 & May & & $3(4)$ & & & & \\
\hline 07 & May & & 1 & & & & \\
\hline 08 & May & & & & & & \\
\hline 09 & May & $3(4)$ & & & & & \\
\hline 10 & May & $4(5)$ & 1 & & $2(3)$ & & \\
\hline 11 & May & 1 & & & & & 1 \\
\hline 12 & May & & & & & & \\
\hline 13 & May & & $2(3)$ & & & & \\
\hline 14 & May & 1 & & & & & \\
\hline 15 & May & $2(3)$ & & & & & 1 \\
\hline 16 & May & & $2(3)$ & & & & 1 \\
\hline & May & & 1 & & & & \\
\hline 18 & May & & & & & 1 & \\
\hline 19 & May & & & & & 1 & \\
\hline 20 & May & 1 & & & & & \\
\hline 21 & May & 1 & & & & 1 & \\
\hline 23 & May & & & & & & \\
\hline
\end{tabular}


Appendix Table 18A. Continued.

\begin{tabular}{|c|c|c|c|c|c|c|}
\hline \multirow[b]{2}{*}{$\begin{array}{c}\text { Detection } \\
\text { date }\end{array}$} & \multirow{3}{*}{$\begin{array}{c}\frac{\text { Lower Granite }}{\text { First }} \\
\text { detection }\end{array}$} & \multicolumn{2}{|c|}{ Little Goose } & \multicolumn{3}{|c|}{ Lower Monumental } \\
\hline & & \multirow[t]{2}{*}{$\begin{array}{l}\text { First } \\
\text { detection }\end{array}$} & \multirow[t]{2}{*}{$\begin{array}{l}\text { Previous } \\
\text { detections } \\
\text { at } 1 \text { dam }\end{array}$} & \multirow[t]{2}{*}{$\begin{array}{l}\text { First } \\
\text { detection }\end{array}$} & \multirow[t]{2}{*}{$\begin{array}{l}\text { Previous } \\
\text { detections } \\
\text { at } 1 \text { dam }\end{array}$} & \multirow[t]{2}{*}{$\begin{array}{l}\text { Previous } \\
\text { detections } \\
\text { at } 2 \text { dams }\end{array}$} \\
\hline 24 May & & & & & & \\
\hline 26 May & 1 & & 1 & & 1 & \\
\hline 28 May & 1 & & & & & \\
\hline 29 May & 1 & & & & & \\
\hline 31 May & & 1 & & & & \\
\hline 01 Jun & & 1 & & & & \\
\hline 03 Jun & & 1 & & & 1 & \\
\hline 04 Jun & & & 1 & & 1 & \\
\hline 06 Jun & 1 & & & & & \\
\hline 08 Jun & 2 & & 1 & & & \\
\hline 09 Jun & & 1 & & & & \\
\hline 10 Jun & $1(2)$ & & 1 & & & \\
\hline 11 Jun & & & & & & 1 \\
\hline 13 Jun & & & & & & \\
\hline 17 Jun & & & & & & 1 \\
\hline 18 Jun & & & & & & \\
\hline 20 Jun & 2 & 1 & & & & \\
\hline 23 Jun & & & 3 & & & \\
\hline 25 Jun & & & 1 & & & \\
\hline
\end{tabular}


Appendix Table 18A. Continued.

\begin{tabular}{|c|c|c|c|c|c|c|}
\hline \multirow[b]{2}{*}{$\begin{array}{c}\text { Detection } \\
\text { date }\end{array}$} & \multirow{2}{*}{$\begin{array}{c}\text { Lower Granite } \\
\text { First } \\
\text { detection }\end{array}$} & \multicolumn{2}{|c|}{ Little Goose } & \multicolumn{3}{|c|}{ Lower Monumental } \\
\hline & & $\begin{array}{c}\text { First } \\
\text { detection }\end{array}$ & $\begin{array}{l}\text { Previous } \\
\text { detections } \\
\text { at } 1 \text { dam }\end{array}$ & $\begin{array}{l}\text { First } \\
\text { detection }\end{array}$ & $\begin{array}{l}\text { Previous } \\
\text { detections } \\
\text { at } 1 \text { dam }\end{array}$ & $\begin{array}{l}\text { Previous } \\
\text { detections } \\
\text { at } 2 \text { dams }\end{array}$ \\
\hline 27 Jun & & & & 1 & & \\
\hline 28 Jun & & 1 & & & & 1 \\
\hline 29 Jun & & 1 & & & & \\
\hline 30 Jun & & & & & 1 & \\
\hline $01 \mathrm{Jul}$ & & & & & 1 & \\
\hline $03 \mathrm{Jul}$ & 1 & & & & & \\
\hline $04 \mathrm{Jul}$ & & & & & & 1 \\
\hline 22 sep & 1 & & & & & \\
\hline Totals & $43 \quad(48)$ & $21 \quad(24)$ & 17 & $7 \quad(8)$ & 19 & 8 \\
\hline
\end{tabular}


Appendix Table 18B. Detections of PIT-tagged smolts by date at three Columbia River dams for wild chinook salmon from the West Fork of Chamberlain Creek, 1995. Numbers in parentheses are first detections at the dams that have been adjusted for spill.

\begin{tabular}{|c|c|c|c|c|c|c|c|c|c|c|c|c|c|c|c|}
\hline \multirow[b]{2}{*}{$\begin{array}{c}\text { Detection } \\
\text { date }\end{array}$} & \multicolumn{4}{|c|}{ McNary } & \multicolumn{5}{|c|}{ John Day } & \multicolumn{6}{|c|}{ Bonneville } \\
\hline & $\begin{array}{c}\text { First } \\
\text { detection }\end{array}$ & \multirow[t]{2}{*}{$\frac{\frac{\text { Previ }}{1}}{\text { dam }}$} & \multirow{2}{*}{$\begin{array}{c}\frac{\text { det }}{2} \\
\text { dams } \\
1\end{array}$} & \multirow[t]{2}{*}{$\begin{array}{l}\frac{\text { tions }}{3} \\
\text { dams }\end{array}$} & $\begin{array}{c}\text { Frrst } \\
\text { detection }\end{array}$ & \multirow{2}{*}{\multicolumn{2}{|c|}{ 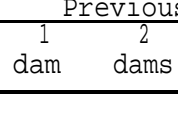 }} & \multirow[t]{2}{*}{$\frac{\frac{\text { etect }}{3}}{\text { dams }}$} & $\frac{\frac{15}{4}}{\text { dams }}$ & $\begin{array}{c}\text { First } \\
\text { detection }\end{array}$ & $\frac{1}{1}$ & \multirow[t]{2}{*}{$\frac{d e r}{2}$} & \multirow[t]{2}{*}{ dams } & \multirow[t]{2}{*}{ dams } & $\begin{array}{c}5 \\
\text { dams }\end{array}$ \\
\hline 02 May & & & & & & & & & & & & & & & \\
\hline 05 May & & 2 & & & & & & & & & & & & & \\
\hline 10 May & & & & & & & & & & & & & & & \\
\hline $11 \mathrm{Ka;}$ & & & & & & & & & & & & & & & \\
\hline 14 Kay & & & & & & & & & & & & & & & \\
\hline 18 May & & 2 & & & & & & & & & & & & & \\
\hline 24 May & & 1 & & & & & & & & & & & & & \\
\hline $10 \mathrm{Jun}$ & & & 1 & & & & & & & & & & & & \\
\hline 18 Jun & & 1 & & & & & & & & & & & & & \\
\hline $27 \mathrm{Jun}$ & $1 \quad(2)$ & & & & & & & & & & & & & & \\
\hline C4 Jul & & & 1 & & & & & & & & & & & & \\
\hline 15 Ju1 & 1 & & & & & & & & & & & & & & \\
\hline Totais & $2 \quad(3)$ & 6 & 6 & & & & & & & & & & & & \\
\hline
\end{tabular}


Appendix Table 19A. Detections of PIT-tagged smolts by date at three Snake River dams for wild chinook salmon from Chamberlain Creek. 1995. Numbers in parentheses are first detections at the dams that have been adjusted for spill.

Tagging site: Chamberlain Creek

Release date: 22 Aug 1994

Release site: Chamberlain Creek

Number released: 241

Release river kilometer(s) above Lower Granite Dam: 438

\begin{tabular}{|c|c|c|c|c|c|c|}
\hline \multirow[b]{2}{*}{$\begin{array}{c}\text { Detection } \\
\text { date }\end{array}$} & \multirow{2}{*}{$\begin{array}{c}\text { Lower Granite } \\
\text { First } \\
\text { detection }\end{array}$} & \multicolumn{2}{|c|}{ Little Goose } & \multicolumn{3}{|c|}{ Lower Monumental } \\
\hline & & $\begin{array}{c}\text { First } \\
\text { detection }\end{array}$ & $\begin{array}{r}\text { Previous } \\
\text { detections } \\
\text { at } 1 \text { dam }\end{array}$ & $\begin{array}{l}\text { First } \\
\text { detection }\end{array}$ & $\begin{array}{l}\text { Previous } \\
\text { detections } \\
\text { at } 1 \text { dam }\end{array}$ & $\begin{array}{r}\text { Previous } \\
\text { detections } \\
\text { at } 2 \text { dams }\end{array}$ \\
\hline 21 Apr & 1 & & & & & \\
\hline 29 Apr & 1 & & & & & \\
\hline 03 May & 1 & & & & & \\
\hline 06 May & & $2(3)$ & & & & \\
\hline 07 May & 1 & $3(4)$ & & & & \\
\hline 08 May & $3 \quad 4)$ & & & 1 & & \\
\hline 09 May & 2 & & & & & \\
\hline 11 May & & 1 & & & & \\
\hline 12 May & 1 & & & & & \\
\hline 13 May & & & & 1 & & 1 \\
\hline 14 May & 1 & & & & & \\
\hline 15 May & & 1 & & 1 & & \\
\hline 18 May & & & & & & \\
\hline 19 May & & & & & & \\
\hline 21 May & & 1 & & & & \\
\hline
\end{tabular}


Appendix Table 19A. Continued.

\begin{tabular}{|c|c|c|c|c|c|c|}
\hline \multirow[b]{2}{*}{$\begin{array}{c}\text { Detection } \\
\text { date }\end{array}$} & \multirow{2}{*}{$\begin{array}{c}\text { Lower Granite } \\
\text { First } \\
\text { detection }\end{array}$} & \multicolumn{2}{|c|}{ Little Goose } & \multicolumn{3}{|c|}{ Lower Monumental } \\
\hline & & $\begin{array}{c}\text { First } \\
\text { detection }\end{array}$ & $\begin{array}{l}\text { Previous } \\
\text { detections } \\
\text { at } 1 \text { dam }\end{array}$ & $\begin{array}{l}\text { First } \\
\text { detection }\end{array}$ & $\begin{array}{l}\text { Previous } \\
\text { detections } \\
\text { at } 1 \text { dam }\end{array}$ & $\begin{array}{l}\text { Previous } \\
\text { detections } \\
\text { at } 2 \text { dams }\end{array}$ \\
\hline 25 May & & & & & 1 & \\
\hline 26 May & & & & 1 & & \\
\hline 03 Jun & & & & 1 & & \\
\hline 07 Jun & & $1(2)$ & & & & \\
\hline 09 Jun & & 1 & & & & \\
\hline 10 Jun & & 1 & & & & \\
\hline 14 Jun & & & & & & \\
\hline 20 Jun & & & & & & \\
\hline 22 Jun & & & & & & \\
\hline 28 Jun & & & & & & \\
\hline 30 Jun & & & & & & \\
\hline $01 \mathrm{Jul}$ & & & & & & 1 \\
\hline $02 \mathrm{Jul}$ & & & & & & 1 \\
\hline $05 \mathrm{Jul}$ & 1 & & & & & \\
\hline Totals & 14 & (14) & 6 & 5 & 8 & 3 \\
\hline
\end{tabular}


Appendix Table 19B. Detections of PIT-tagged smolts by date at three Columbia River dams for wild chinook salmon from Chamberlain Creek. 1995. Numbers in parentheses are first detections at the dams that have been adjusted for spill.

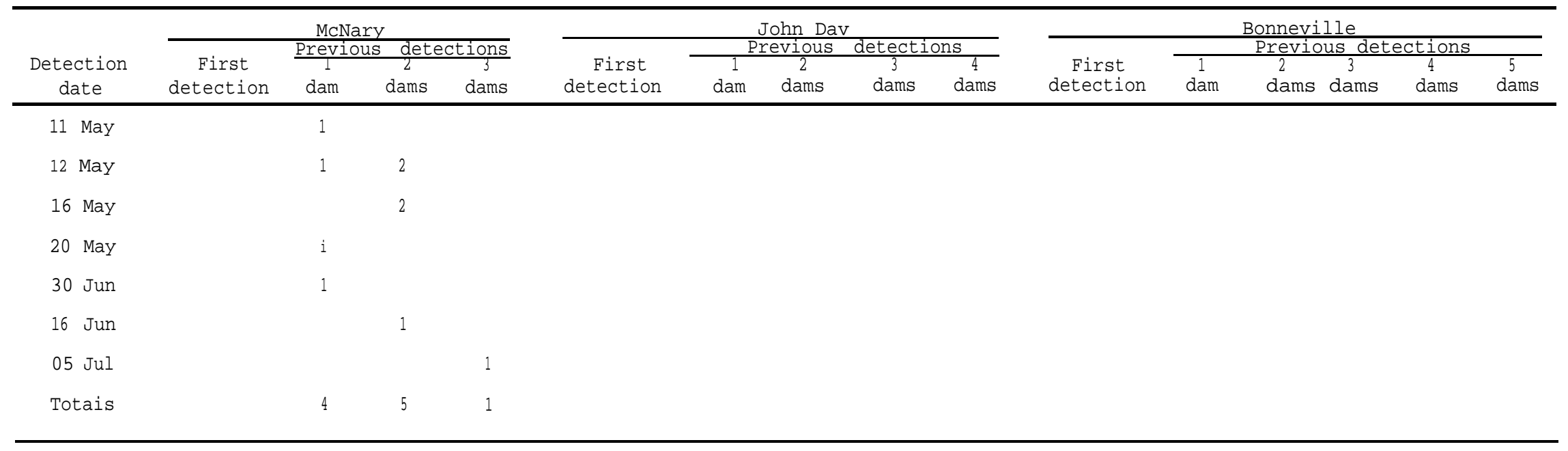


Appendix Table 20A. Detections of PIT-tagged smolts by date at three Snake River dams for wild chinook salmon from Secesh River. 1995. Numbers in parentheses are first detections at the dams that have been adjusted for spill.

Tagging site: Secesh River

Release date: 23 - 24 Aug 1994

Release site: Secesh River

Number released: 1.551

Release river kilometer(s) above Lower Granite Dam: 430 - 432

\begin{tabular}{|c|c|c|c|c|c|c|}
\hline \multirow[b]{2}{*}{$\begin{array}{c}\text { Detection } \\
\text { date }\end{array}$} & \multirow{3}{*}{$\begin{array}{c}\text { Lower Granite } \\
\begin{array}{c}\text { First } \\
\text { detection }\end{array} \\
2\end{array}$} & \multicolumn{2}{|c|}{ Little Goose } & \multicolumn{3}{|c|}{ Lower Monumental } \\
\hline & & $\begin{array}{c}\text { First } \\
\text { detection }\end{array}$ & $\begin{array}{l}\text { Previous } \\
\text { detections } \\
\text { at } 1 \text { dam }\end{array}$ & $\begin{array}{l}\text { First } \\
\text { detection }\end{array}$ & $\begin{array}{l}\text { Previous } \\
\text { detections } \\
\text { at } 1 \text { dam }\end{array}$ & $\begin{array}{l}\text { Previous } \\
\text { detections } \\
\text { at } 2 \text { dams }\end{array}$ \\
\hline 10 Apr & & & & & & \\
\hline 12 Apr & 2 & & & & & \\
\hline 13 Apr & 4 & 1 & & & & \\
\hline 14 Apr & 2 & & & & & \\
\hline 15 Apr & 6 & & & & & \\
\hline 16 Apr & 3 & 1 & & & & \\
\hline 17 Apr & 2 & 1 & & & & \\
\hline 18 Apr & 3 & & & & & \\
\hline 19 Apr & 2 & 1 & & & & \\
\hline 20 Apr & 1 & & & & & \\
\hline 21 Apr & 4 & & & & & \\
\hline 22 Apr & 3 & $1(2)$ & & & & \\
\hline 23 Apr & 1 & $1(2)$ & & & & \\
\hline 24 Apr & 2 & & & & & \\
\hline 26 Apr & 1 & 1 & & 1 & & \\
\hline
\end{tabular}


Appendix Table 20A. Continued

\begin{tabular}{|c|c|c|c|c|c|c|c|}
\hline \multirow{2}{*}{\multicolumn{2}{|c|}{$\begin{array}{l}\text { Detection } \\
\text { date }\end{array}$}} & \multirow{2}{*}{$\begin{array}{c}\text { Lower Granite } \\
\text { First } \\
\text { detection }\end{array}$} & \multicolumn{2}{|c|}{ Little Goose } & \multicolumn{3}{|c|}{ Lower Monumental } \\
\hline & & & $\begin{array}{l}\text { First } \\
\text { detection }\end{array}$ & \multirow{2}{*}{$\begin{array}{r}\text { Previous } \\
\text { detections } \\
\text { at } 1 \text { dam } \\
1\end{array}$} & $\begin{array}{l}\text { First } \\
\text { detection }\end{array}$ & $\begin{array}{l}\text { Previous } \\
\text { detections } \\
\text { at } 1 \text { dam }\end{array}$ & $\begin{array}{l}\text { Previous } \\
\text { detections } \\
\text { at } 2 \text { dams }\end{array}$ \\
\hline 27 & Apr & & & & 1 & 1 & \\
\hline 28 & Apr & 1 & 1 & & & 1 & \\
\hline 29 & Apr & 2 & & & & 2 & \\
\hline 30 & Apr & 4 & 1 & 1 & & 2 & \\
\hline 01 & May & 5 & $3(4)$ & & $3(4)$ & & \\
\hline 02 & May & 1 & & 1 & & & \\
\hline 03 & May & 3 & 1 & 1 & 1 & 1 & \\
\hline 04 & May & & 1 & & 1 & & 2 \\
\hline 05 & May & $2(3)$ & 1 & 1 & & 3 & \\
\hline 06 & May & $5(6)$ & $3(4)$ & 3 & 1 & & \\
\hline 07 & May & & & 1 & & 1 & 1 \\
\hline 08 & May & & & 1 & 1 & 3 & 1 \\
\hline 09 & May & $3(4)$ & & 1 & 1 & & \\
\hline 10 & May & $5(6)$ & 1 & & 1 & 1 & \\
\hline 11 & May & 1 & $3(4)$ & 2 & & & 2 \\
\hline 12 & May & $3(4)$ & $4(5)$ & & 1 & 2 & \\
\hline 13 & May & & $1(2)$ & & $3(4)$ & 1 & 1 \\
\hline 14 & May & & & & & 3 & \\
\hline 15 & May & & 1 & & $2(3)$ & & \\
\hline
\end{tabular}


Appendix Table 20A. Continued.

\begin{tabular}{|c|c|c|c|c|c|c|}
\hline \multirow[b]{2}{*}{$\begin{array}{l}\text { Detection } \\
\text { date }\end{array}$} & \multirow{2}{*}{$\begin{array}{c}\text { Lower Granite } \\
\text { First } \\
\text { detection }\end{array}$} & \multicolumn{2}{|c|}{ Little Goose } & \multicolumn{3}{|c|}{ Lower Monumental } \\
\hline & & $\begin{array}{c}\text { First } \\
\text { detection }\end{array}$ & $\begin{array}{r}\text { Previous } \\
\text { detections } \\
\text { at } 1 \text { dam }\end{array}$ & $\begin{array}{l}\text { First } \\
\text { detection }\end{array}$ & $\begin{array}{l}\text { Previous } \\
\text { detections } \\
\text { at } 1 \text { dam }\end{array}$ & $\begin{array}{r}\text { Previous } \\
\text { detections } \\
\text { at } 2 \text { dams }\end{array}$ \\
\hline 16 May & & & 1 & & 1 & \\
\hline 17 May & & 1 & & 2 & 1 & \\
\hline 18 May & & & 1 & 1 & & 1 \\
\hline 20 May & & & & 1 & 1 & \\
\hline 21 May & 1 & 1 & & & & \\
\hline 22 May & & $2(3)$ & & 1 & & \\
\hline 24 May & $3(4)$ & 1 & & & & \\
\hline 25 May & 1 & & & 1 & & \\
\hline 27 May & 1 & & & & & \\
\hline 28 May & 1 & 2 & & & & \\
\hline 29 May & & 1 & & & & \\
\hline 31 May & & & & i & & \\
\hline 01 Jun & 1 & 1 & & & & 1 \\
\hline $03 \mathrm{Jun}$ & & & & & & \\
\hline 04 Jun & & 1 & & & & \\
\hline 06 Jun & 1 & & & & & \\
\hline 07 Jun & 2 & & & 1 & & \\
\hline 08 Jun & & & & & & 1 \\
\hline 09 Jun & & 1 & & & & \\
\hline
\end{tabular}


Appendix Table 20A. Continued.

\begin{tabular}{|c|c|c|c|c|c|c|}
\hline \multirow[b]{2}{*}{$\begin{array}{c}\text { Detection } \\
\text { date }\end{array}$} & \multirow{2}{*}{$\begin{array}{c}\text { Lower Granite } \\
\text { First } \\
\text { detection }\end{array}$} & \multicolumn{2}{|c|}{ Little Goose } & \multicolumn{3}{|c|}{ Lower Monumental } \\
\hline & & $\begin{array}{c}\text { First } \\
\text { detection }\end{array}$ & $\begin{array}{l}\text { Previous } \\
\text { detections } \\
\text { at } 1 \text { dam }\end{array}$ & $\begin{array}{l}\text { First } \\
\text { detection }\end{array}$ & $\begin{array}{l}\text { Previous } \\
\text { detections } \\
\text { at } 1 \text { dam }\end{array}$ & $\begin{array}{l}\text { Previous } \\
\text { detections } \\
\text { at } 2 \text { dams }\end{array}$ \\
\hline 10 Jun & & & 1 & & & \\
\hline 11 Jun & & 1 & & & & \\
\hline 12 Jun & & & & & & 1 \\
\hline 13 Jun & & & & & & \\
\hline 14 Jun & & & & & & \\
\hline 18 Jun & & & & & & \\
\hline 21 Jun & & & & & & \\
\hline 22 Jun & & 1 & & 1 & & \\
\hline 24 Jun & & & & 1 & & \\
\hline 28 Jun & & & & & & \\
\hline $10 \mathrm{Jul}$ & & & & & & \\
\hline 13 Jul & & 2 & & & & \\
\hline $15 \mathrm{Jul}$ & & 1 & & & & \\
\hline $26 \mathrm{Jul}$ & & & & & 1 & \\
\hline $27 \mathrm{Jul}$ & & & & 1 & & \\
\hline Totals & $86 \quad(92)$ & $44 \quad(52)$ & 28 & $28 \quad(31)$ & 36 & 11 \\
\hline
\end{tabular}


Appendix Table 20B. Detections of PIT-tagged smolts by date at three Columbia River dams for wild chinook salmon from the Secesh River. 1995. Numbers in parentheses are first detections at the dams that have been adjusted for spill.

\begin{tabular}{|c|c|c|c|c|c|c|c|c|c|c|c|c|c|c|c|c|c|}
\hline \multirow{2}{*}{\multicolumn{2}{|c|}{$\begin{array}{l}\text { Detection } \\
\text { date }\end{array}$}} & \multicolumn{5}{|c|}{$\begin{array}{c}\text { YCNary } \\
\text { Previous }\end{array}$} & \multicolumn{5}{|c|}{ John Day } & \multicolumn{6}{|c|}{ Bonneville } \\
\hline & & \multicolumn{2}{|c|}{$\begin{array}{c}\text { First } \\
\text { detection }\end{array}$} & \multirow[t]{2}{*}{$\begin{array}{l}\frac{\text { Previ }}{1} \\
\text { dam }\end{array}$} & \multirow[t]{2}{*}{$\begin{array}{c}2 \\
\text { dams }\end{array}$} & $\overline{3}$ & $\begin{array}{c}\text { First } \\
\text { detection }\end{array}$ & \multicolumn{2}{|c|}{\begin{tabular}{cc} 
& \multicolumn{2}{c}{ Previous } \\
1 & 2 \\
dam & dams \\
\end{tabular}} & \multirow[t]{2}{*}{$\begin{array}{c}\text { detectr } \\
3 \\
\text { dams }\end{array}$} & $\frac{\frac{1 s}{4}}{4 a m s}$ & $\begin{array}{c}\text { First } \\
\text { detection }\end{array}$ & $\begin{array}{c}1 \\
\text { darn }\end{array}$ & $\begin{array}{c}\text { Previou } \\
2 \\
\text { dans }\end{array}$ & \multirow[t]{2}{*}{$\begin{array}{l}\text { dis det } \\
3 \\
\text { dams }\end{array}$} & \multirow[t]{2}{*}{$\begin{array}{c}\text { Ctions } \\
4 \\
\text { darns }\end{array}$} & $\begin{array}{c}5 \\
\text { dams } \\
\end{array}$ \\
\hline 25 & Apr & & & & & & & & & & & & & & & & \\
\hline 27 & Apr & & & 1 & & & & & & & & & & & & & \\
\hline 28 & Apr & 1 & & 1 & & & & & & & & & & & & & \\
\hline \multicolumn{18}{|c|}{$30 \mathrm{Apr}$} \\
\hline 01 & May & 2 & 3 & 1 & & & & & & & & & & & & & \\
\hline 02 & May & & & 3 & & & & & & & & & & & & & \\
\hline 04 & May & & & 1 & & & & & & & & & & & & & \\
\hline 07 & May & & & 1 & & 1 & & & & & & & & & & & \\
\hline 09 & May & & & & & 1 & & & & & & & & & & & \\
\hline 10 & May & & & & 2 & 1 & & & & & & & & & & & \\
\hline 11 & May & 1 & $2:$ & 1 & & & & & & & & & & & & & \\
\hline 12 & May & & & & 4 & 1 & & & & & & & & & & & \\
\hline \multicolumn{18}{|c|}{13 May } \\
\hline \multicolumn{18}{|c|}{15 Yav } \\
\hline 16 & y & & & 3 & 1 & 1 & & & & & & & & & & & \\
\hline & May & & & 1 & 2 & & & & & & & & & & & & \\
\hline 18 & MaY & & & & & 1 & & & & & & & & & & & \\
\hline 19 & May & & & & & & & & 1 & & & & & & & & \\
\hline 21 & May & & & 1 & & & & & & & & & & & & & \\
\hline 22 & May & & & & & & & & & & & & & & & & \\
\hline 23 & May & 1 & 2 & 1 & & & & & & & & & & & & & \\
\hline 25 & May & & & & $=$ & & & & & & & & & & & & \\
\hline
\end{tabular}


Appendix Table 20B. Continued.

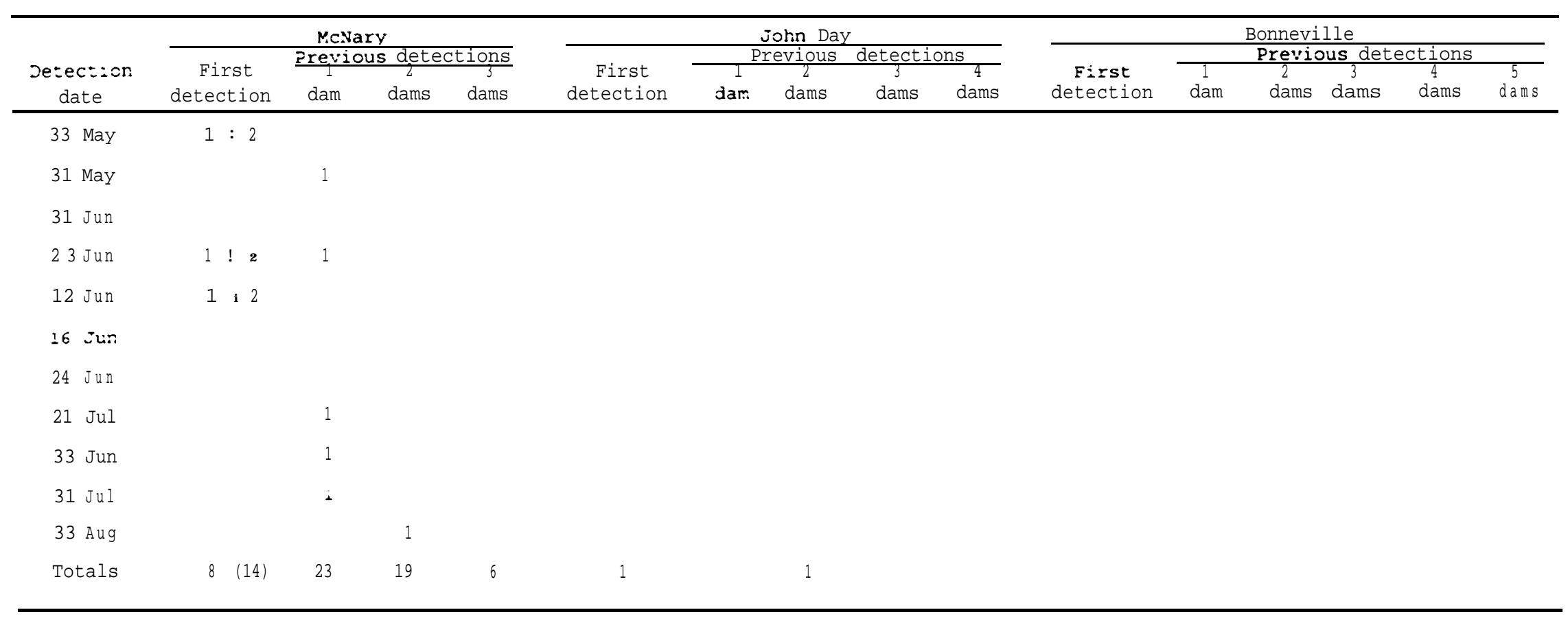


Appendix Table 21 A. Detections of PIT-tagged smolts by date at three Snake River dams for wild chinook salmon from Lake Creek. 1995. Numbers in parentheses are first detections at the dams that have been adjusted for spill.

Tagging site: Lake Creek

Release date: 24 Aug 1994

Release site: Lake Creek

Number released: 405

Release river kilometer(s) above Lower Granite Dam: 452

\begin{tabular}{|c|c|c|c|c|c|c|}
\hline \multirow[b]{2}{*}{$\begin{array}{c}\text { Detection } \\
\text { date }\end{array}$} & \multirow{3}{*}{$\begin{array}{c}\text { Lower Granite } \\
\begin{array}{c}\text { First } \\
\text { detection }\end{array} \\
1\end{array}$} & \multicolumn{2}{|c|}{ Little Goose } & \multicolumn{3}{|c|}{ Lower Monumental } \\
\hline & & $\begin{array}{c}\text { First } \\
\text { detection }\end{array}$ & $\begin{array}{l}\text { Previous } \\
\text { detections } \\
\text { at } 1 \text { dam }\end{array}$ & $\begin{array}{l}\text { First } \\
\text { detection }\end{array}$ & $\begin{array}{l}\text { Previous } \\
\text { detections } \\
\text { at } 1 \text { dam }\end{array}$ & $\begin{array}{l}\text { Previous } \\
\text { detections } \\
\text { at } 2 \text { dams }\end{array}$ \\
\hline 14 Apr & & & & & & \\
\hline 16 Apr & 1 & & & & & \\
\hline 17 Apr & 2 & & & & & \\
\hline 18 Apr & 1 & & & & & \\
\hline 21 Apr & & & 1 & & & \\
\hline 23 Apr & 1 & & & & & \\
\hline 25 Apr & 1 & & & & & \\
\hline 27 Apr & 1 & & 1 & & & \\
\hline 28 Apr & & & 1 & & & \\
\hline 29 Apr & & 1 & & & & \\
\hline 01 May & 1 & & 1 & & 1 & \\
\hline 02 May & 1 & & & & & \\
\hline 03 May & & 1 & & & & \\
\hline 04 May & 1 & & & & & \\
\hline 05 May & & 1 & 1 & & 1 & \\
\hline
\end{tabular}


Appendix Table 2 I A. Continued.

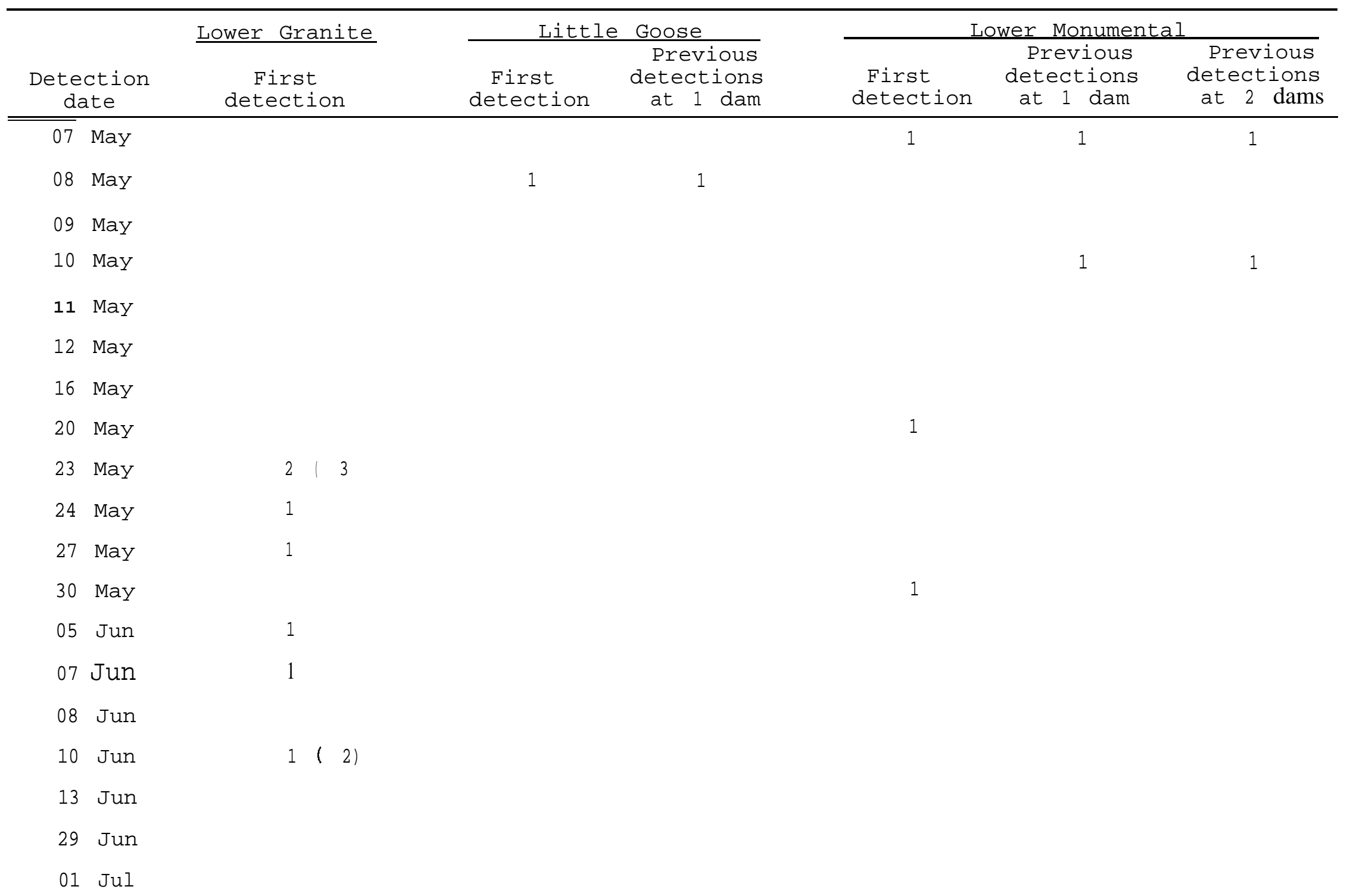


Appendix Table 2 I A. Continued.

\begin{tabular}{|c|c|c|c|c|c|c|}
\hline \multirow[b]{2}{*}{$\begin{array}{c}\text { Detection } \\
\text { date }\end{array}$} & \multirow{2}{*}{$\begin{array}{c}\text { Lower Granite } \\
\text { First } \\
\text { detection }\end{array}$} & \multicolumn{2}{|c|}{ Little Goose } & \multicolumn{3}{|c|}{ Lower Monumental } \\
\hline & & $\begin{array}{c}\text { First } \\
\text { detection }\end{array}$ & $\begin{array}{l}\text { Previous } \\
\text { detections } \\
\text { at } 1 \text { dam }\end{array}$ & $\begin{array}{l}\text { First } \\
\text { detection }\end{array}$ & $\begin{array}{l}\text { Previous } \\
\text { detections } \\
\text { at } 1 \text { dam }\end{array}$ & $\begin{array}{l}\text { Previous } \\
\text { detections } \\
\text { at } 2 \text { dams }\end{array}$ \\
\hline $20 \mathrm{Jul}$ & 1 & & & & & \\
\hline 09 Aug & & & & 1 & & \\
\hline Totals & $(26)$ & 6 & 8 & 4 & 6 & 3 \\
\hline
\end{tabular}


Appendix Table 2 I B. Detections of PIT-tagged smolts by date at three Columbia River dams for wild chinook salmon from Lake Creek. 1995. Numbers in parentheses are first detections at the dams that have been adjusted for spill.

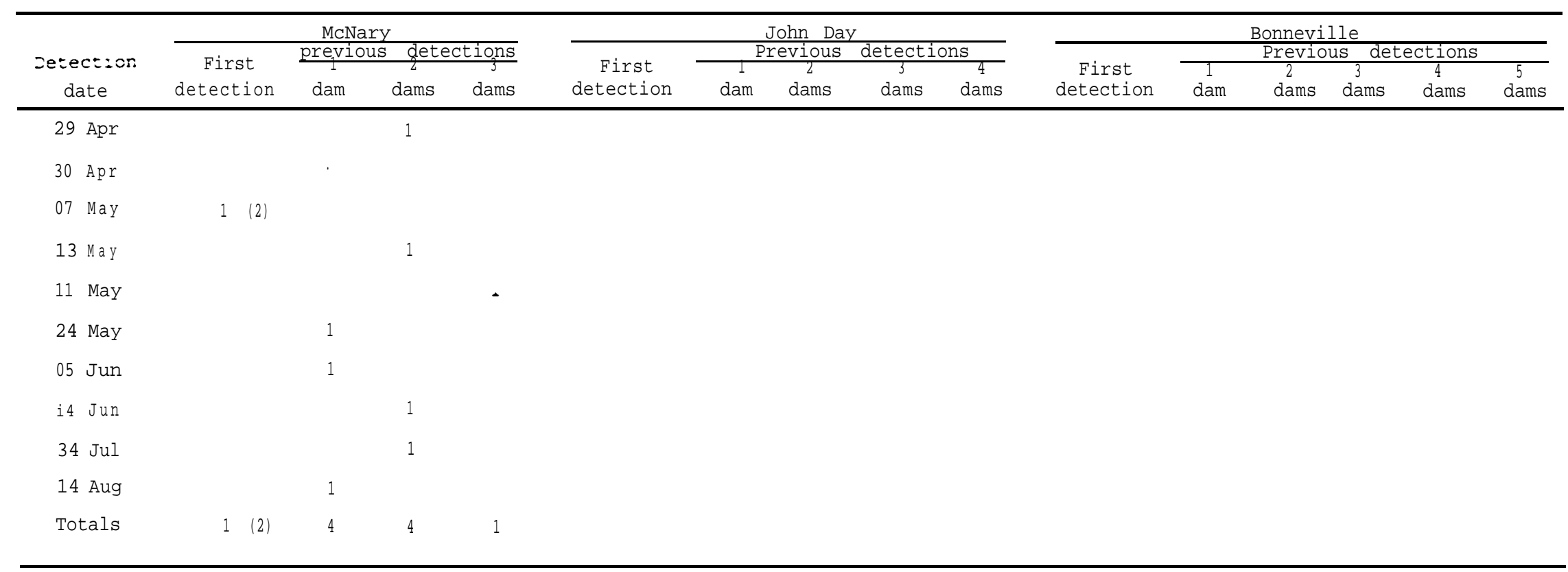


Appendix Table 22. A summary of the tagging dates, start tagging times and temperatures $\left({ }^{\circ} \mathrm{C}\right)$, release dates, times, and temperatures, methods of capture, distance (in kilometers) from the stream's mouth to the release point, number released, unadjusted number detected, and unadjusted percent detected for each tag group at six downstream dams during 1995.

\begin{tabular}{|c|c|c|c|c|c|c|c|c|c|c|c|c|}
\hline Stream & Tag group & $\begin{array}{l}\text { Tagging } \\
\text { date }\end{array}$ & $\begin{array}{l}\text { Tagging } \\
\text { time }\end{array}$ & $\begin{array}{l}\text { Release } \\
\text { date }\end{array}$ & $\begin{array}{l}\text { Release } \\
\text { time }\end{array}$ & $\begin{array}{l}\text { Tagging } \\
\text { temp. }\left({ }^{\circ} \mathrm{C}\right)\end{array}$ & $\begin{array}{l}\text { Release } \\
\text { temp. }\left({ }^{\circ} \mathrm{C}\right)\end{array}$ & $\begin{array}{l}\text { Capture } \\
\text { method }\end{array}$ & $\begin{array}{l}\text { Release } \\
\text { River km }\end{array}$ & $\begin{array}{l}\text { Number } \\
\text { Released }\end{array}$ & $\begin{array}{l}\text { Number } \\
\text { detected }\end{array}$ & $\begin{array}{l}\text { Percent } \\
\text { detected (t) }\end{array}$ \\
\hline \multirow[t]{11}{*}{ Bear Valley Creek } & SA94208.BVI & $21 \mathrm{Jul}$ & 07.39 & $27 \mathrm{Jul}$ & 09:30 & 15.5 & 15.5 & Shock & 9 & 90 & 9 & 10.0 \\
\hline & SA9420S.BV2 & $27 \mathrm{Jul}$ & 0836 & $27 \mathrm{JuI}$ & $10: 00$ & 15.5 & 15.5 & Shock & 10 & 125 & 13 & 10.4 \\
\hline & SA9420S.BW & $27 \mathrm{J"} 1$ & 095 & $28 \mathrm{Jul}$ & 06:00 & 16.0 & 16.0 & Shock & 11 & 18 & 9 & 11.5 \\
\hline & SA94209.BVI & $28 \mathrm{Jul}$ & $06: 31$ & 28 JU1 & 08.30 & 15.5 & 15.5 & Shock & 11 & 70 & 4 & 5.1 \\
\hline & SA94209.BV2 & 28 JU1 & $07: 27$ & 28 JU1 & $10: 00$ & 15.5 & 16.0 & Shock & 12 & 211 & 15 & 7.1 \\
\hline & SA94209.BV3 & 28 JUI & OS: 25 & 29 Jul & $06: 30$ & 16.0 & 15.5 & Shock & 12 & 153 & 10 & 6.5 \\
\hline & SA94209.BV4 & $28 \mathrm{Jul}$ & $09: 44$ & $29 \mathrm{Jul}$ & $0-I: 30$ & 16.0 & 15.5 & Shock & 13 & 215 & 12 & 5.6 \\
\hline & SA94210.BV1 & $29 \mathrm{Jul}$ & $06: 5-1$ & 29 JU1 & 08:00 & 15.5 & 15.5 & Shock & 13 & 119 & 8 & 6.7 \\
\hline & SA94210.BV2 & $29 \mathrm{Jul}$ & $07: 36$ & $29 \mathrm{Jul}$ & 09:30 & 15.5 & 15.5 & Seine & 14 & 132 & 9 & 6.8 \\
\hline & SA94210.BV3 & $29 \mathrm{Jul}$ & os: 32 & $29 \mathrm{Jul}$ & $09: 30$ & 15.5 & 15.5 & Seine & 14 & 150 & 13 & 6.7 \\
\hline & SA94210.BV4 & 29 Jul & $09: 20$ & 29 Jul & $10: 30$ & 15.5 & 16.5 & Shock & 14 & 112 & 5 & 4.5 \\
\hline \multirow[t]{10}{*}{ Elk Creek } & SA94210.EC1 & 29 Jul & $09: 39$ & $30 \mathrm{Jul}$ & $-^{-}$ & - & - & Seine & 1 & 159 & 17 & 10.7 \\
\hline & KMC94211.EC1 & $30 \mathrm{Jul}$ & $11: 32$ & $30 \mathrm{Jul}$ & 13.30 & 16.0 & 17.0 & Shock & 1 & 100 & 4 & 4.0 \\
\hline & KMC94211.ЕС2 & $30 \mathrm{Jul}$ & $12: 21$ & $31 \mathrm{Jul}$ & oe:०० & 16.5 & 14.0 & Shock & 1 & 67 & 10 & 14.9 \\
\hline & KMC94212.BC1 & $31 \mathrm{Jul}$ & $06: 27$ & $31 \mathrm{Jul}$ & - & 14.5 & 15.0 & Shock & 1 & & 10 & 5.3 \\
\hline & КМС 94212. ВC2 & 31 Jul & OS: 21 & 31 Jul & $12: 00$ & 14.0 & 17.5 & Shock & 2 & 111 & 12 & 10.3 \\
\hline & $\mathrm{mc} 94212$ BC3 & 31 Jul & $09: 35$ & $31 \mathrm{Jul}$ & 12.00 & 15.5 & 17.5 & Shock & 2 & 156 & 17 & 10.9 \\
\hline & КМС 94212. ВС 4 & $31 \mathrm{Jul}$ & 11.13 & 01 Aug & $06: 30$ & 17.0 & - & Shock & 2 & 162 & 12 & 7.4 \\
\hline & KMC94213.EC1 & 01 Aug & $07: 39$ & 01 xug & 09:30 & 14.0 & 15.0 & Shock & 3 & $3 s$ & 5 & 13.2 \\
\hline & $\mathrm{mlC} 94213 . \mathrm{BC2}$ & 01 Aug & OS: 01 & 01 Aug & 10 :so & 14.5 & 15.5 & Shock & 3 & 398 & 27 & 6.9 \\
\hline & KMC94213.ВC3 & $01 \mathrm{Ag}$ & $10: 10$ & $01 \mathrm{Ag}$ & $11: 30$ & 15.0 & 16.0 & Shock & 4 & 126 & 8 & 6.3 \\
\hline
\end{tabular}


Appendix Table 22. Continued.

\begin{tabular}{|c|c|c|c|c|c|c|c|c|c|c|c|c|}
\hline stream & Tag group & $\begin{array}{l}\text { Tagging } \\
\text { date }\end{array}$ & $\begin{array}{l}\text { Tagging } \\
\text { time }\end{array}$ & $\begin{array}{l}\text { RelCFISe } \\
\text { date }\end{array}$ & $\begin{array}{l}\text { Release } \\
\text { time }\end{array}$ & $\begin{array}{l}\text { Tagging } \\
\text { temp. }\left({ }^{\circ} \mathrm{C}\right)\end{array}$ & $\begin{array}{l}\text { Release } \\
\text { temp. }\left({ }^{\circ} \mathrm{C}\right)\end{array}$ & $\begin{array}{l}\text { capture } \\
\text { method }\end{array}$ & $\begin{array}{l}\text { Release } \\
\text { River } \mathbf{k m}\end{array}$ & $\begin{array}{l}\text { Number } \\
\text { Released }\end{array}$ & $\begin{array}{l}\text { Number } \\
\text { detected }\end{array}$ & $\begin{array}{l}\text { Percent } \\
\text { detected ( }\end{array}$ \\
\hline \multirow[t]{6}{*}{ Sulphur Creek } & SA94212.SUl & $31 \mathrm{JuI}$ & 08:30 & 31 Jul & $09: 30$ & 11.0 & 11.0 & Seine & 5 & 42 & 7 & 16.7 \\
\hline & SA94212.SU2 & 31 Jul & $10: 00$ & 01 Aug & --- & 11.0 & - $^{-}$ & shock & 5 & 15 & 11 & 14.7 \\
\hline & SA94212.SU3 & $31 \mathrm{~J} \mathrm{ul}$ & $12: 17$ & 01 Aug & 07:00 & 13.0 & 11.0 & Shock & 6 & 198 & 18 & 9.1 \\
\hline & SA94213, SU1 & 01 Aug & 08:14 & 01 Aug & $09: 30$ & 11.0 & 11.5 & Shock & 6 & 124 & 22 & 17.7 \\
\hline & SA94213.S42 & 01 Aug & $10: 00$ & 01 Aug & $12: 00$ & 11.5 & 13.5 & Shock & -- & 101 & 18 & 17.8 \\
\hline & SA94213.SU3 & 01 Aug & $11: 29$ & 01 Aug & $13: 30$ & 12.5 & 14.5 & Shock & 7 & 188 & 35 & 18.6 \\
\hline \multirow[t]{5}{*}{ Marsh Creek } & SA94214.MCI & 02 Aug & 07:43 & 02 Aug & $12: 00$ & 9.0 & 12.5 & seine & 14 & 383 & 39 & 10.2 \\
\hline & SA94214.MC2 & 02 Aug & $09: 37$ & $03 \quad$ A"g & $\ldots$ & 9.5 & $\ldots$ & Shock & 11 & 128 & 14 & 10.9 \\
\hline & SA94214.MC3 & 02 Aug & $10: 58$ & 02 Aug & $12: 30$ & 11.5 & 13.0 & Shock & 32 & 178 & 19 & 10.7 \\
\hline & SA94214.MC4 & 02 Aug & $11: 39$ & 02 Aug & $13: 30$ & 12.5 & 15.5 & Seine & 14 & 406 & 45 & 11.1 \\
\hline & КMC94214.MCI & 02 A"g & 12:03 & 02 Aug & $13: 30$ & $12.5-$ & 15.5 & 8eine & 14 & 480 & 51 & 10.6 \\
\hline \multirow[t]{5}{*}{ Cape Horn Creek } & $\mathrm{SA} 94215 . \mathrm{CH} 1$ & 03 Aug & $07: 36$ & 03 Aug & 11:oo & 9.5 & 12.5 & Seille & 6 & 295 & 27 & 9.2 \\
\hline & $\mathrm{SA} 94215 . \mathrm{CH} 2$ & 03 Aug & $09: 32$ & 04 A"g & $06: 30$ & 10.0 & 9.5 & SdII.2 & 6 & 175 & 20 & 11.4 \\
\hline & $\mathrm{SA} 94215 . \mathrm{CH} 3$ & 03 Aug & $10: 24$ & 03 Aug & $13: 00$ & 11.0 & 14.5 & Seine & 6 & 454 & 50 & 11.0 \\
\hline & SA94215.CH4 & 03 Aug & $12: 39$ & $03 \mathrm{Aug}$ & $13: 30$ & 14.5 & 14.5 & Shock & 6 & 60 & 4 & 6.7 \\
\hline & KMC94215.CIil & 03 Aug & $12: 16$ & $03 \mathrm{Aug}$ & 13:30 & 13.0 & 14.5 & Seine & 6 & 459 & 47 & 10.2 \\
\hline \multirow[t]{4}{*}{ Valley Creek } & SA94216.VCl & 04 Aug & 08:11 & $04 \mathrm{Aug}$ & $10: 30$ & 11.5 & 14.5 & Shock & 4 & 675 & 36 & 5.3 \\
\hline & SA94216.VCZ & 04 Aug & 10:29 & $05 \mathrm{Aug}$ & 08:30 & 14.5 & 11.0 & Shock & 4 & 111 & 6 & 5.4 \\
\hline & SA94217.vCl & 05 A"g & o-J:53 & 05 Aug & $10: 00$ & 10.0 & 13.0 & Shock & 18 & 55 & 3 & 5.5 \\
\hline & SA94217.VC2 & 05 Aug & 08:15 & 05 Aug & $10: 30$ & 11.0 & 13.0 & seine & $\cdots$ & 711 & 50 & 7.0 \\
\hline
\end{tabular}


Appendix Table 22. Continued.

\begin{tabular}{|c|c|c|c|c|c|c|c|c|c|c|c|c|}
\hline stream & Tag group & $\begin{array}{l}\text { Tagging } \\
\text { date }\end{array}$ & $\begin{array}{l}\text { Tagging } \\
\text { time }\end{array}$ & $\begin{array}{l}\text { Release } \\
\text { date }\end{array}$ & $\begin{array}{l}\text { Release } \\
\text { time }\end{array}$ & $\begin{array}{l}\text { Tagging } \\
\text { temp. }\left({ }^{\circ} \mathrm{C}\right)\end{array}$ & $\begin{array}{l}\text { Release } \\
\text { temp. }\left({ }^{\circ} \mathrm{C}\right)\end{array}$ & $\begin{array}{l}\text { capture } \\
\text { method }\end{array}$ & $\begin{array}{l}\text { Release } \\
\text { River } \mathrm{km}\end{array}$ & $\begin{array}{l}\text { Number } \\
\text { Released }\end{array}$ & $\begin{array}{l}\text { Number } \\
\text { detected }\end{array}$ & $\begin{array}{l}\text { Percent } \\
\text { detected ( }(t)\end{array}$ \\
\hline \multirow[t]{7}{*}{ camas Creek } & SA94220.CAI & 08 Aug & $11: 32$ & 09 Aug & $06: 30$ & 11.0 & 11.0 & Seme & 22 & 43 & 7 & 16.2 \\
\hline & SA94220.CA2 & 08 Aug & $12: 01$ & 09 Aug & $09: 00$ & 12.0 & 10.5 & Shock & 22 & 499 & 47 & 9.4 \\
\hline & SA94221.CAI & 09 Aug & $06: 49$ & 09 Aug & $12: 30$ & 11.0 & 14.5 & Shock & 23 & 73 & 11 & 15.1 \\
\hline & SA94221.CA2 & 09 Aug & $08: 18$ & 09 Aug & $13: 15$ & 10.5 & 15.0 & Shock & 20 & 298 & 19 & 6.3 \\
\hline & SA94221.CA3 & 09 Aug & $10: 52$ & 10 Aug & $06: 30$ & 11.5 & 10.0 & Shock & 21 & 266 & 14 & 5.1 \\
\hline & $\mathrm{SA} 94222 . \mathrm{CAI}$ & 10 Rug & $08: 32$ & 10 Aug & $09: 45$ & 9.5 & 10.5 & Shock & 22 & 20 & - & - \\
\hline & SA94222.CA2 & 10 Aug & $08: 45$ & 10 Aug & $10: 00$ & 9.5 & 10.5 & Shock & 21 & 329 & 24 & 7.3 \\
\hline \multirow[t]{5}{*}{ Loon Creek } & DJK94221.LN1 & 09 Aug & $08: 50$ & $09 \mathrm{~A} " \mathrm{~g}$ & $12: 45$ & 9.5 & 12.0 & Shock & 33 & 303 & 40 & 13.2 \\
\hline & DJK94221.LN2 & 09 Aug & $12: 41$ & 09 Aug & $14: 15$ & 9.0 & 12.0 & Shock & 33 & 196 & 33 & 16.8 \\
\hline & DJK94221.LN3 & 09 Aug & $13: 21$ & 09 Aug & $14: 15$ & 9.0 & 12.0 & Shock & 34 & 148 & 28 & 18.9 \\
\hline & DJK94221.LN4 & 09 Aug & $13: 55$ & 09 Aug & $14: 45$ & 9.0 & 12.0 & Shock & 35 & 132 & 21 & 15.9 \\
\hline & DJX94221.W5 & 09 Aug & $14: 15$ & 09 Aug & $15: 15$ & 12.0 & 12.0 & Shock & 35 & 185 & 33 & 17.8 \\
\hline \multirow[t]{2}{*}{ Herd Creek } & $\mathrm{SA} 94224 . \mathrm{HCl}$ & 12 Aug & $10: 39$ & 12 Aug & $12: 00$ & 12.0 & 12.5 & Shock & 0 & 311 & 40 & 12.7 \\
\hline & SA94225. HC1 & 13 Aug & $09: 44$ & 13 Aug & II $: 45$ & 12.0 & 14.0 & Shock & 2 & 223 & 25 & 11.2 \\
\hline \multirow[t]{4}{*}{ B. F. Salmon River } & SA94224 .BP1 & 12 Aug & 09:01 & 12 Aug & 11:00 & 11.5 & 12.5 & Shock & 13 & 270 & 20 & 7.4 \\
\hline & SA94224.BF2 & 12 Aug & $11: 58$ & 12 Aug & 13:०0 & 14.0 & 14.0 & Shock & 14 & 173 & 22 & 12.7 \\
\hline & SA94225.BPI & 13 Aug & $08: 41$ & 13 Aug & $11: 30$ & 11.0 & 14.0 & Shock & 15 & 253 & 23 & 9.1 \\
\hline & SA94225.BP2 & 13 Aug & $\mathrm{VI}: 46$ & 13 Aug & $12: 00$ & 13.0 & 14.5 & Shock & 16 & 290 & 30 & 10.3 \\
\hline \multirow[t]{3}{*}{ Big Creek (upper) } & MBB94229. BC1 & 17 Aug & $07: 42$ & 17 Aug & $-^{-}$ & 8.0 & 14.0 & Seine & 52 & 358 & 38 & 10.6 \\
\hline & PAO94229. BC2 & 17 Aug & $11: 22$ & 17 Aug & ----- & 12.0 & 14.0 & Shock & 53 & 216 & 34 & 15.7 \\
\hline & MBE94229.BC3 & 17 Aug & $13: 13$ & 17 Aug & ${ }^{-}$ & ---- & - & Shock & 55 & 183 & 21 & 11.5 \\
\hline
\end{tabular}


Appendix Table 22. Continued.

\begin{tabular}{|c|c|c|c|c|c|c|c|c|c|c|c|c|}
\hline Stream & Tag group & $\begin{array}{l}\text { Tagging } \\
\text { date }\end{array}$ & $\begin{array}{l}\text { Tagging } \\
\text { time }\end{array}$ & $\begin{array}{l}\text { Release } \\
\text { date }\end{array}$ & $\begin{array}{l}\text { Release } \\
\text { time }\end{array}$ & $\begin{array}{l}\text { Tagging } \\
\text { temp. }\left({ }^{\circ} \mathrm{C}\right)\end{array}$ & $\begin{array}{l}\text { Release } \\
\text { temp. }\left({ }^{\circ} \mathrm{C}\right)\end{array}$ & $\begin{array}{l}\text { capture } \\
\text { method }\end{array}$ & $\begin{array}{l}\text { Release } \\
\text { River km }\end{array}$ & $\begin{array}{l}\text { Number } \\
\text { Released }\end{array}$ & $\begin{array}{l}\text { Number } \\
\text { detected }\end{array}$ & $\begin{array}{l}\text { Percent } \\
\text { detected ( }\end{array}$ \\
\hline \multirow[t]{5}{*}{ S. P. Salmon River } & SA94228.SFI & 16 Aug & $07: 57$ & 16 Aug & $11: 30$ & 12.0 & 14.0 & Seine & 112 & 367 & 24 & 6.5 \\
\hline & SA94228.SF2 & 16 Aug & $10: 52$ & 16 Aug & $12: 30$ & 13.0 & 14.5 & Seine & 113 & 328 & 20 & 6.1 \\
\hline & SA94229.SFI & 17 Aug & $10: 12$ & $1.8 \mathrm{Aug}$ & ----- & 10.0 & ---- & seine & 117 & 54 & 7 & 13.0 \\
\hline & SA94229.SF2 & 17 Aug & $12: 54$ & $18 \mathrm{Ang}$ & …. & 13.5 & - & Shock & 122 & 313 & 36 & 11.5 \\
\hline & SA94230.SPI & 1 Aug & 08:05 & 18 Aug & $12: 15$ & 9.0 & 112.5 & Shock & 123 & 507 & 39 & 7.7 \\
\hline \multirow[t]{3}{*}{ Big Creek (lower) } & DJK94233.BCI & 21 Aug & $09: 28$ & 21 Aug & $, \times 3: 00$ & 13.0 & 15.0 & Shock & 10 & 262 & 71 & 27.1 \\
\hline & D) K94233.BCZ & 21 Aug & 11:00 & 21 Aug & 13:20 & 14.0 & 15.0 & Shock & 11 & 210 & 52 & 24.8 \\
\hline & DJK94233.BC3 & 21 Aug & $11: 47$ & $21 \mathrm{Aug}$ & $----=$ & 14.0 & 15.0 & Shock & 13 & 255 & 55 & 21.6 \\
\hline Rush Creek & DJK94233.RCI & 21 Aug & $13: 30$ & $21 \mathrm{~A} " \mathrm{~g}$ & $14: 00$ & 15.0 & 15.0 & Shock & 1 & 15 & 4 & 26.7 \\
\hline \multirow[t]{2}{*}{ w. P. Chamberlain Creek } & SA94233.WCI & 21 Aug & 08:35 & 21 Aug & $11: 30$ & 9.0 & 11.5 & Seine & 1 & 450 & 40 & 8.9 \\
\hline & SA94233.WC2 & $21 \mathrm{~A} " \mathrm{~g}$ & 10:44 & 21 Aug & $13: 00$ & 10.5 & 13.5 & seine & 2 & 467 & 33 & 7.1 \\
\hline Chamberlain Creek & SA94234 .CBI & 22 Aug & $11: 46$ & 22 Aug & $13: 45$ & 10.5 & 11.5 & Shock & 26 & 241 & 30 & 12.4 \\
\hline \multirow[t]{3}{*}{ Secesh River } & SA94235.SBI & 23 Aug & $09: 46$ & 23 Aug & 13:45 & 7.0 & 15.0 & Shock & 26 & 405 & 56 & 13.8 \\
\hline & SA94235.SB2 & 23 Aug & 11:29 & 23 A"g & 14:45 & 10.0 & 15.0 & Shock & 27 & 810 & 75 & 9.3 \\
\hline & KMC94236.SBI & 24 Aug & $09: 55$ & 24 Aug & $12: 00$ & 9.0 & 11.0 & Shock & 28 & 336 & 36 & 10.7 \\
\hline Lake Creek & KMC94236.LCI & 24 A"g & $12: 11$ & 24 Aug & $15: 00$ & 11.0 & 13.5 & Shock & 3 & 405 & 35 & 8.6 \\
\hline
\end{tabular}


Appendix Table 23. Daily detections of PIT-tagged wild spring/summer chinook salmon smolts from Idaho at Lower Granite Dam during 1995, with associated river flows (kcfs), spill (kcfs), and water temperatures $\left({ }^{\circ} \mathrm{C}\right)$ at the dam. Adjusted numbers detected are calculated during spill.

\begin{tabular}{|c|c|c|c|c|c|c|}
\hline \multicolumn{2}{|c|}{ Date } & \multirow{2}{*}{\begin{tabular}{c}
\multicolumn{2}{c}{ Average } \\
flow (kcfs) \\
46.9
\end{tabular}} & \multirow{2}{*}{ 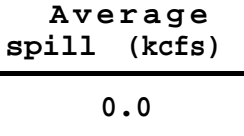 } & \multirow{2}{*}{$\begin{array}{c}\begin{array}{c}\text { Scroll-case water } \\
\text { temperature } \\
\left({ }^{\circ} \mathrm{C}\right)\end{array} \\
9.4\end{array}$} & \multirow{2}{*}{$\frac{\begin{array}{c}\text { Numbers } \\
\text { detected }\end{array}}{0}$} & \multirow{2}{*}{$\begin{array}{c}\text { Adjusted } \\
\text { numbers detected } \\
0\end{array}$} \\
\hline 07 & Apr & & & & & \\
\hline 08 & Apr & 60.1 & 0.0 & 10.0 & 0 & 0 \\
\hline 09 & Apr & 71.1 & 0.0 & 10.0 & 0 & 0 \\
\hline 10 & Apr & 64.2 & 0.0 & 10.0 & 3 & 3 \\
\hline 11 & Apr & 68.2 & 0.0 & & 8 & 8 \\
\hline 12 & Apr & 65.1 & 0.0 & 10.0 & 10 & 10 \\
\hline 13 & Apr & 61.2 & 0.0 & 10.0 & 20 & 20 \\
\hline 14 & Apr & 73.4 & 0.0 & 10.0 & 13 & 13 \\
\hline 15 & Apr & 70.3 & 0.0 & 8.9 & 15 & 15 \\
\hline 16 & Apr & 69.8 & 0.0 & 8.9 & 15 & 15 \\
\hline 17 & Apr & 52.0 & 1.8 & ---- & 18 & 19 \\
\hline 18 & Apr & 64.5 & 0.0 & 9.4 & 17 & 17 \\
\hline 19 & Apr & 64.2 & 0.0 & ---- & 13 & 13 \\
\hline 20 & Apr & 64.4 & 0.0 & 9.4 & 18 & 18 \\
\hline 21 & Apr & 63.6 & 0.0 & 9.4 & 24 & 24 \\
\hline 22 & Apr & 59.3 & 0.0 & 9.4 & 8 & 8 \\
\hline 23 & Apr & 52.5 & 0.0 & 9.4 & 7 & 7 \\
\hline 24 & Apr & 53.7 & 0.0 & 9.4 & 8 & 8 \\
\hline ' 25 & Apr & 60.1 & 0.0 & 9.4 & 9 & 9 \\
\hline 26 & Apr & 67.2 & 0.0 & 9.4 & 18 & 18 \\
\hline 27 & Apr & 72.5 & 0.0 & 10.6 & 21 & 21 \\
\hline 28 & Apr & 73.9 & 0.0 & 10.6 & 30 & 30 \\
\hline 29 & Apr & 79.4 & 0.0 & ---- & 23 & 23 \\
\hline 30 & Apr & 84.1 & 0.0 & & 14 & 14 \\
\hline 01 & May & 81.1 & 0.0 & 10.6 & 33 & 33 \\
\hline 02 & May & 88.4 & 0.0 & 10.6 & 33 & 33 \\
\hline 03 & May & 93.1 & 9.9 & 10.0 & 30 & 34 \\
\hline 04 & May & 94.7 & 20.7 & 10.0 & 28 & 36 \\
\hline 05 & May & 96.9 & 21.7 & 10.6 & 15 & 19 \\
\hline 06 & May & 98.9 & $\begin{array}{ll}2 & 1\end{array}$ & 10.6 & 16 & 21 \\
\hline
\end{tabular}


Appendix Table 23. Continued.

\begin{tabular}{|c|c|c|c|c|c|c|c|c|}
\hline \multicolumn{2}{|c|}{ Date } & \multirow{2}{*}{$\frac{\begin{array}{c}\text { Average } \\
\text { flow } \quad(\text { kcfs) }\end{array}}{118.1}$} & \multirow{2}{*}{$\begin{array}{c}\begin{array}{c}\text { Average } \\
\text { spill }\end{array} \\
23.5\end{array}$} & \multirow{2}{*}{$\begin{array}{r}\begin{array}{c}\text { Scroll-case } \\
\text { temperature }\end{array} \\
10.6\end{array}$} & \multirow{2}{*}{$\frac{\begin{array}{l}\text { Numbers } \\
\text { detected }\end{array}}{17}$} & \multicolumn{3}{|c|}{$\begin{array}{c}\text { Adjusted } \\
\text { numbers detected }\end{array}$} \\
\hline 07 & May & & & & & & 21 & \\
\hline 08 & May & 128.8 & $27 \cdot 4$ & 10.0 & 11 & & 14 & \\
\hline 09 & May & 114.8 & 22.0 & 10.0 & 67 & & 83 & \\
\hline 10 & May & 115.0 & 21.9 & 10.6 & 45 & & 56 & \\
\hline 11 & May & 118.2 & 24.9 & 10.6 & 32 & & 41 & \\
\hline 12 & May & 119.6 & 21.8 & 10.6 & 22 & & 27 & \\
\hline 13 & May & 112.4 & 21.7 & 11.7 & 23 & & 29 & \\
\hline 14 & May & 101.9 & 21.8 & 11.1 & 32 & & 41 & \\
\hline 15 & May & 95.6 & 21.9 & 11.1 & 17 & & 22 & \\
\hline 16 & May & 101.4 & 21.9 & 11.7 & 9 & & $\begin{array}{ll}1 & 1\end{array}$ & \\
\hline 17 & May & 106.2 & 21.7 & $11.7^{\prime}$ & 5 & & 6 & \\
\hline 18 & May & 109.4 & 22.2 & 12.2 & 10 & & 13 & \\
\hline 19 & May & 112.7 & 24.3 & 12.8 & 7 & & 9 & \\
\hline 20 & May & 114.7 & 27.4 & 12.8 & 15 & & 20 & \\
\hline 21 & May & 116.1 & 27.4 & 13.3 & 11 & & 14 & \\
\hline 22 & May & 118.5 & 27.3 & 13.3 & 3 & & 4 & \\
\hline 23 & May & 120.5 & 27.3 & 13.3 & 13 & & 1 & 7 \\
\hline 24 & May & 117.5 & 27.4 & 13.3 & 20 & & 26 & \\
\hline 25 & May & 116.5 & 19.9 & 13.3 & 13 & & 16 & \\
\hline 26 & May & 111.9 & 19.8 & 13.3 & 7 & & 9 & \\
\hline 27 & May & 108.3 & 6.3 & 13.3 & 26 & & 28 & \\
\hline 28 & May & 109.0 & 6.4 & 13.3 & 21 & & 22 & \\
\hline 29 & May & 102.0 & 6.4 & 13.3 & 19 & & 20 & \\
\hline 30 & May & 110.9 & 6.5 & 13.9 & 5 & & 5 & \\
\hline 31 & May & 122.6 & 6.8 & 15.0 & 7 & & 7 & \\
\hline 01 & Jun & 133.0 & 10.9 & 15.0 & 16 & & 17 & \\
\hline 02 & Jun & 133.6 & 10.0 & 15.0 & 6 & & 6 & \\
\hline 03 & Jun & 133.8 & 11.3 & 15.0 & 11 & & 12 & \\
\hline 04 & Jun & 141.2 & 13.4 & 15.0 & 7 & & 8 & \\
\hline 05 & Jun & 144.0 & 17.4 & 15.0 & 13 & & 15 & \\
\hline 06 & Jun & 149.1 & 25.4 & 15.0 & 13 & & 16 & \\
\hline 07 & Jun & 145.8 & 23.5 & 15.0 & 14 & & 17 & \\
\hline 08 & Jun & 130.6 & 11.8 & 13.3 & 6 & & 7 & \\
\hline
\end{tabular}


Appendix Table 23. Continued.

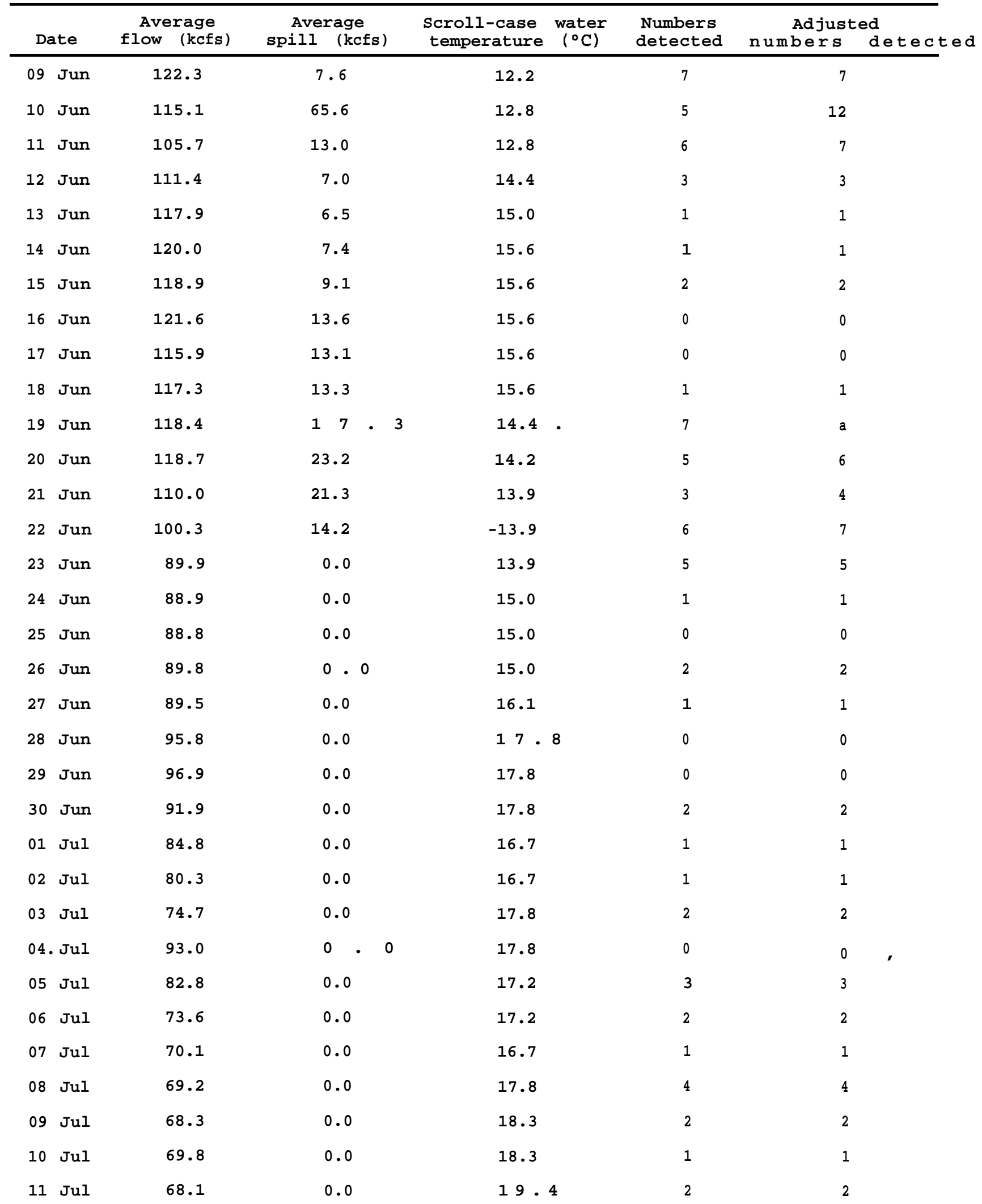


Appendix Table 23. Continued.

\begin{tabular}{|c|c|c|c|c|c|}
\hline Date & $\begin{array}{l}\text { Average } \\
\text { flow (kcfs) }\end{array}$ & \begin{tabular}{cc}
\multicolumn{2}{c}{ Avkrage } \\
spill & (kcfs)
\end{tabular} & $\begin{array}{l}\text { Scroll-case water } \\
\text { temperature }\left({ }^{\circ} \mathrm{C}\right)\end{array}$ & $\begin{array}{l}\text { Numbers } \\
\text { detected }\end{array}$ & $\begin{array}{c}\text { Adjusted } \\
\text { numbers detected }\end{array}$ \\
\hline $12 \mathrm{Jul}$ & 69.1 & 0.0 & 19.4 & 0 & 0 \\
\hline $13 \mathrm{Jul}$ & 66.3 & 0.0 & 19.4 & 3 & 3 \\
\hline $14 \mathrm{Jul}$ & 66.0 & 0.0 & 19.4 & 0 & 0 \\
\hline $15 \mathrm{Jul}$ & 56.8 & 0.0 & 18.9 & 1 & 1 \\
\hline $16 \mathrm{Jul}$ & 54.8 & 0.0 & 18.9 & 0 & 0 \\
\hline $17 \mathrm{Jul}$ & 50.9 & 0.0 & 21.1 & 0 & 0 \\
\hline $18 \mathrm{Jul}$ & 54.9 & 0.0 & 19.4 & 2 & 2 \\
\hline $19 \mathrm{Jul}$ & 51.8 & 0.0 & 19.4 & 0 & 0 \\
\hline $20 \mathrm{Jul}$ & 49.7 & 0.0 & 19.4 & 1 & 1 \\
\hline $21 \mathrm{Jul}$ & 48.5 & 0.0 & 19.4 & 0 & 0 \\
\hline $22 \mathrm{Jul}$ & 49.2 & 0.0 & 19.4 & 1 & 1 \\
\hline $23 \mathrm{Jul}$ & 45.5 & 0.0 & 20.0 & 0 & 0 \\
\hline $24 \mathrm{Jul}$ & 45.8 & $0: 0$ & 20.0 & 0 & 0 \\
\hline $25 \mathrm{Jul}$ & 51.8 & 0.0 & 20.0 & 0 & 0 \\
\hline $26 \mathrm{Jul}$ & 50.9 & 0.0 & 20.9 & 0 & 0 \\
\hline $27 \mathrm{Jul}$ & 50.1 & 0.0 & 20.0 & 0 & 0 \\
\hline $28 \mathrm{Jul}$ & 47.2 & 0.0 & 20.0 & 1 & 1 \\
\hline $29 \mathrm{Jul}$ & 50.5 & 0.0 & 20.0 & 0 & 0 \\
\hline 22 Sep & 26.7 & 0.0 & 19.9 & 1 & 1 \\
\hline
\end{tabular}




\section{6}

Appendix Table 24. Daily detections of PIT-tagged wild spring/summer chinook salmon smolts from Idaho at Little Goose Dam during. 1995, with associated river flows (kcfs), spill (kcfs), and water temperatures $\left({ }^{\circ} \mathrm{C}\right)$ at the dam. Numbers detected represent fish not detected- at a previous dam. Adjusted numbers detected are calculated during spill.

\begin{tabular}{|c|c|c|c|c|c|c|c|}
\hline \multicolumn{2}{|c|}{ Date } & \multirow{2}{*}{\begin{tabular}{c}
\multicolumn{2}{c}{$\begin{array}{c}\text { Average } \\
\text { flow (kcfs) }\end{array}$} \\
64.0
\end{tabular}} & \multirow{2}{*}{$\begin{array}{c}\text { Average } \\
\text { spill (kcfs) } \\
0.0\end{array}$} & \multirow{2}{*}{$\begin{array}{r}\begin{array}{r}\text { Scroll, -case } \\
\text { temperature }\end{array} \\
6.1\end{array}$} & \multirow{2}{*}{$\begin{array}{c}\text { water } \\
\left({ }^{\circ} \mathrm{C}\right)\end{array}$} & \multirow{2}{*}{$\frac{\begin{array}{c}\text { Numbers } \\
\text { detected }\end{array}}{0}$} & \multirow{2}{*}{$\begin{array}{c}\text { Adjusted } \\
\text { numbers detected } \\
0\end{array}$} \\
\hline 10 & Apr & & & & & & \\
\hline 11 & Apr & 74.9 & 0.0 & $-\cdots$ & & $0^{\prime}$ & 0 \\
\hline 12 & Apr & 61.4 & 0.0 & 6.7 & & 0 & 0 \\
\hline 13 & Apr & 63.6 & 0.0 & 7.2 & & 1 & 1 \\
\hline 14 & Apr & 74.7 & 2.0 & 7.2 & & 0 & 0 \\
\hline 15 & Apr & 80.1 & 6.4 & 10.0 & & 2 & 2 \\
\hline 16 & Apr & 76.2 & 8.0 & 7.8 & & 1 & 1 \\
\hline 17 & Apr & 49.0 & 2.8 & ---- & & 6 & 6 \\
\hline 18 & Apr & 63.9 & 12.2 & 8.9 & & 1 & 1 \\
\hline 19 & Apr & 61.7 & 14.4 & 9.4 & & 1 & 1 \\
\hline 20 & Apr & 73.9 & 17.7 & 9.4 & & 0 & 0 \\
\hline 21 & Apr & 68.1 & 20.2 & 9.4 & & 0 & 0 \\
\hline 22 & Apr & 62.6 & 21.0 & 9.4 & & 3 & 5 \\
\hline 23 & Apr & 51.2 & 20.2 & 9.4 & & 3 & 5 \\
\hline 24 & Apr & 53.3 & 21.2 & 10.0 & & 0 & 0 \\
\hline 25 & Apr & 63.5 & 21.8 & 10.0 & & 1 & 2 \\
\hline 26 & Apr & 65.7 & 21.7 & 10.0 & & 2 & 3 \\
\hline 27 & Apr & 72.4 & 20.4 & 10.0 & & 0 & 0 \\
\hline 28 & Apr & 74.3 & 19.5 & 10.0 & & 4 & 5 \\
\hline 29 & Apr & 81.6 & 19.1 & ---- & & 4 & 5 \\
\hline 30 & Apr & 85.4 & 19.3 & ---- & & $8^{\prime}$ & 10 \\
\hline 01 & May & 81.0 & 19.3 & 10.6 & & 8 & 11 \\
\hline 02 & May & 83.6 & 20.1 & 10.6 & & 8 & 11 \\
\hline 03 & May & 91.8 & 21.1 & 10.6 & & 8 & 10 \\
\hline 04 & May & 97.9 & 22.7 & 10.6 & & 9 & 12 \\
\hline 05 & May & 97.7 & 23.9 & 10.6 & & 10 & 13 \\
\hline 06 & May & 96.9 & 24.3 & 10.0 & & 22 & 29 \\
\hline 07 & May & 115.9 & 33.1 & 10.6 & & 16 & 22 \\
\hline 08 & May & 126.7 & 39.2 & 11.1 & & 15 & 22 \\
\hline 09 & May & 113.5 & 35.0 & 11.1 & & 8 & 12 \\
\hline
\end{tabular}


Appendix Table 24. Continued.

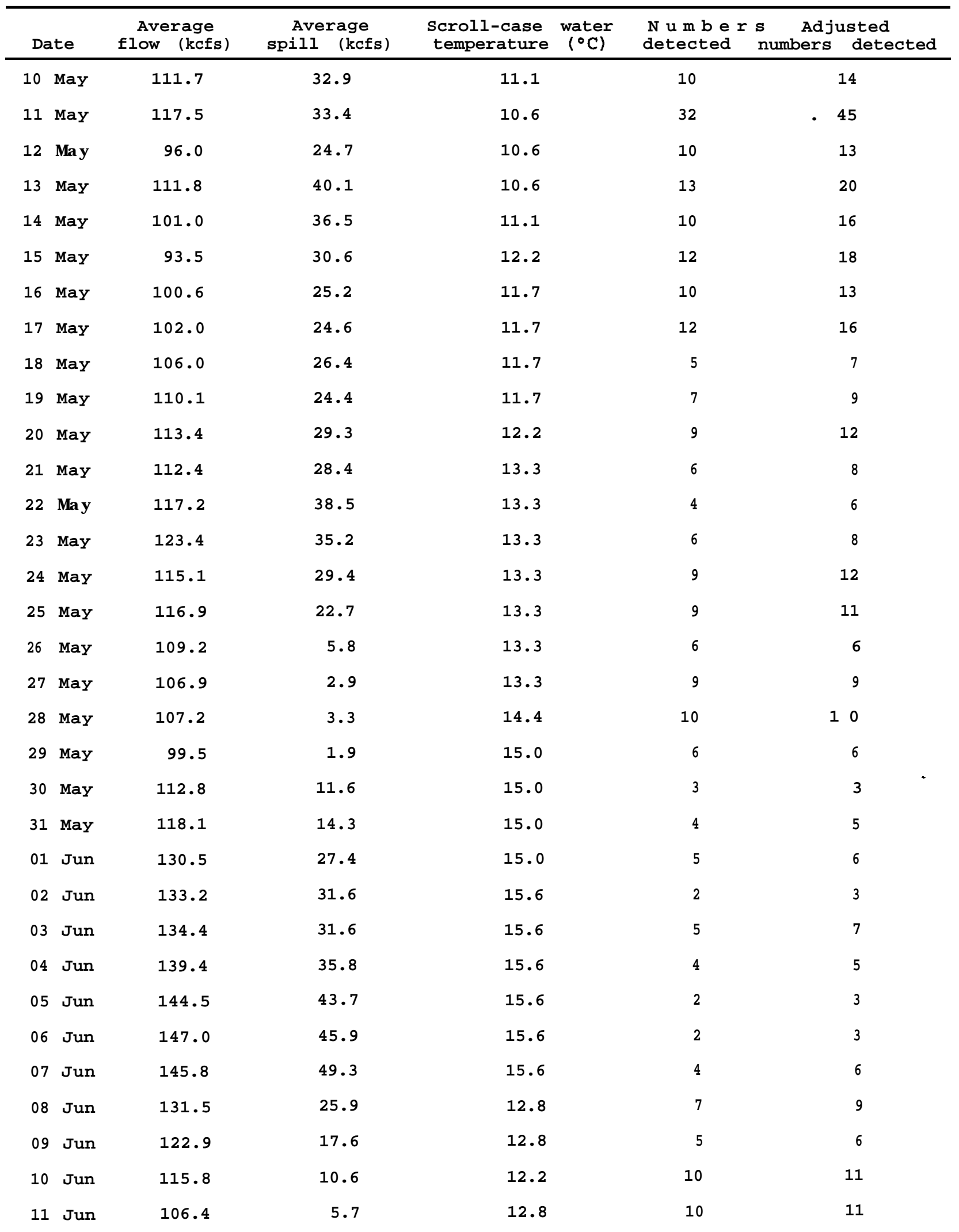


Appendix Table 24. Continued.

\begin{tabular}{|c|c|c|c|c|c|c|c|}
\hline \multicolumn{2}{|c|}{ Date } & \multirow{2}{*}{$\begin{array}{c}\text { Average } \\
\text { flow (kcfs) }\end{array}$} & \multirow{2}{*}{\begin{tabular}{c}
\multicolumn{2}{c}{ Average } \\
spill (kcfs)
\end{tabular}} & \multirow{2}{*}{$\begin{array}{c}\begin{array}{c}\text { Scroll-case } \\
\text { temperature }\end{array} \\
12.8\end{array}$} & \multirow[t]{2}{*}{$\begin{array}{c}\text { water } \\
\left({ }^{\circ} \mathrm{C}\right)\end{array}$} & \multirow{2}{*}{$\begin{array}{c}\begin{array}{c}\text { Numbers } \\
\text { detected }\end{array} \\
4\end{array}$} & \multirow{2}{*}{\begin{tabular}{c}
\multicolumn{2}{c}{ Adjusted } \\
numbers \\
4
\end{tabular}} \\
\hline 12 & Jun & & & & & & \\
\hline 13 & Jun & 118.1 & 12.9 & 13.3 & & 3 & 3 \\
\hline 14 & Jun & 116.6 & 22.6 & 13.9 & & 4 & 5 \\
\hline 15 & Jun & 119.0 & 19.0 & 14.4 & & 1 & 1 \\
\hline 16 & Jun & 121.3 & 20.3 & 15.6 & & 2 & 2 \\
\hline 17 & Jun & 117.0 & 15.1 & 15.6 & & 1 & 1 \\
\hline 18 & Jun & 117.6 & 16.1 & 15.6 & & 1 & 1 \\
\hline 19 & Jun & 117.9 & 17.5 & 15.6 & & 0 & 0 \\
\hline 20 & Jun & 117.8 & 17.0 & 14.4 & & 1 & 1 \\
\hline 21 & Jun & 111.7 & 12.1 & 14 & . 4 & 1 & 1 \\
\hline 22 & Jun & 101.5 & 10.2 & 13.9 & & 2 & 2 \\
\hline 23 & Jun & 90.3 & 10.4 & 15.6 & & 2 & 2 \\
\hline 24 & Jun & 92.0 & 14.2 & 15.6 & & 2 & 2 \\
\hline 25 & Jun & 89.3 & 19.1 & 15.6 & & 1 & 1 \\
\hline 26 & Jun & 89.3 & 11.8 & 15.6 & & 0 & 0 \\
\hline 27 & Jun & 90.9 & 0.0 & 15.6 & & 0 & 0 \\
\hline 28 & Jun & 97.6 & 6.0 & 15.6 & & 3 & 3 \\
\hline 29 & Jun & 102.0 & 11.6 & 15.6 & & 5 & 6 \\
\hline 30 & Jun & 92.7 & 0.0 & 16.1 & & 1 & 1 \\
\hline 01 & Jul & 86.4 & 0.0 & 17.2 & & 2 & 2 \\
\hline 02 & Jul & 81.7 & 0.0 & 17.8 & & 0 & 0 \\
\hline 03 & Jul & 74.3 & 0.0 & 17.8 & & 0 & 0 \\
\hline 04 & Jul & 91.2 & 0.0 & 17.8 & & 0 & 0 \\
\hline 05 & Jul & 87.7 & 0.0 & 18.3 & & 1 & 1 \\
\hline 06 & Jul & 75.8 & 0.0 & 17 & 8 & 0 & 0 \\
\hline 07 & Jul & 71.0 & 0.0 & 18.3 & & 0 & 0 \\
\hline 08 & Jul & 73.6 & 0.0 & 18 & 3 & 1 & 1 \\
\hline 09 & Jul & 67.7 & $0.1)$ & 17.8 & & 2 & 2 \\
\hline 10 & Jul & 74.6 & 0.0 & 17.8 & & 0 & 0 \\
\hline 11 & Jul & 72.3 & 0.0 & 18.9 & & 0 & 0 \\
\hline 12 & Jul & 73.9 & 0.0 & 19.4 & & 0 & 0 \\
\hline 13 & Jul & 71.6 & 0.0 & 18.9 & & 3 & 3 \\
\hline 14 & Jul &, 67.6 & 0.0 & 19.4 & & 0 & 0 \\
\hline
\end{tabular}


Appendix Table 24. Continued.

\begin{tabular}{|c|c|c|c|c|c|}
\hline Date & $\begin{array}{l}\text { Average } \\
\text { flow (kcfs) }\end{array}$ & $\begin{array}{c}\text { Average } \\
\text { spill } \\
\text { (kcfs) }\end{array}$ & $\begin{array}{c}\text { Scroll-case water } \\
\text { temperature }\left({ }^{\circ} \mathrm{C}\right)\end{array}$ & $\begin{array}{l}\text { Numbers } \\
\text { detected }\end{array}$ & $\begin{array}{c}\text { Adjusted } \\
\text { numbers detected }\end{array}$ \\
\hline $15 \mathrm{Jul}$ & 57.0 & 0.0 & 20.0 & 1 & 1 \\
\hline $16 \mathrm{Jul}$ & 56.5 & 0.0 & 21.7 & 0 & 0 \\
\hline $17 \mathrm{Jul}$ & 51.4 & 0.0 & 21.1 & 0 & 0 \\
\hline $18 \mathrm{Jul}$ & 56.4 & 0.0 & 22.2 & 0 & 0 \\
\hline $19 \mathrm{Jul}$ & 52.7 & 0.0 & 21.1 & 0 & 0 \\
\hline $20 \mathrm{Jul}$ & 50.4 & 0.0 & 20.6 & 0 & 0 \\
\hline $21 \mathrm{Jul}$ & 49.1 & 0.0 & 20.6 & 1 & 1 \\
\hline $22 \mathrm{Jul}$ & 48.8 & 0.0 & 21.1 & 1 & 1 \\
\hline $23 \mathrm{Jul}$ & 46.6 & 0.0 & 21.1 & 0 & 0 \\
\hline $24 \mathrm{Jul}$ & 46.5 & 0.0 & 21.1 & 0 & 0 \\
\hline $25 \mathrm{Jul}$ & 49.8 & 0.0 & 21.1 & 0 & 0 \\
\hline $26 \mathrm{Jul}$ & 51.2 & 0.0 & ---- & 0 & 0 \\
\hline $27 \mathrm{Jul}$ & 50.2 & 0.0 & 21.1 & 0 & 0 \\
\hline $28 \mathrm{Jul}$ & 48.4 & 0.0 & 21.1 & 0 & 0 \\
\hline $29 \mathrm{Jul}$ & 51.5 & 0.0 & 21.1 & 0 & 0 \\
\hline $30 \mathrm{Jul}$ & 46.1 & 0.0 & 21.1 & 0 & 0 \\
\hline $31 \mathrm{Jul}$ & 46.3 & 0.0 & 21.1 & 0 & 0 \\
\hline 01 Aug & 46.5 & 0.0 & 21.1 & 0 & 0 \\
\hline 02 Aug & 45.9 & 0.0 & 21.1 & 0 & 0 \\
\hline 03 Aug & 45.1 & 0.0 & 21.1 & 0 & 0 \\
\hline 04 Aug & 44.7 & 0.0 & 21.1 & 0 & 0 \\
\hline 05 Aug & 45.1 & 0.0 & 20.6 & 0 & 0 \\
\hline 06 Aug & 41.5 & 0.0 & 20.6 & 0 & 0 \\
\hline 07 Aug & 44.9 & 0.0 & 20.6 & 0 & 0 \\
\hline 08 Aug & 42.8 & 0.0 & 20.0 & 0 & 0 \\
\hline 09 Aug & 41.3 & 0.0 & 21.1 & 0 & 0 \\
\hline 10 Aug & 42.3 & 0.0 & 20.0 & 1 & 1 \\
\hline 11 Aug & 34.1 & 0.0 & 19.4 & 0 & 0 \\
\hline 12 Aug & 35.5 & 0.0 & 20.5 & 0 & 0 \\
\hline 13 Aug & 33.3 & 0.0 & 20.0 & 0 & 0 \\
\hline 14 Aug & 34.3 & 0.0 & 20.0 & 0 & 0 \\
\hline 15 Aug & 35.2 & 0.0 & 20.0 & 0 & 0 \\
\hline 16 Aug & 34.4 & 0.0 & 19.4 & 0 & 0 \\
\hline
\end{tabular}


Appendix Table 24. Continued.

\begin{tabular}{|c|c|c|c|c|c|c|c|}
\hline \multicolumn{2}{|c|}{ Date } & \multirow{2}{*}{$\begin{array}{c}\begin{array}{c}\text { Average } \\
\text { flow }(\text { kcfs })\end{array} \\
35.0\end{array}$} & \multirow{2}{*}{$\begin{array}{c}\text { Average } \\
\text { spill (kcfs) } \\
0.0\end{array}$} & \multirow{2}{*}{$\begin{array}{r}\begin{array}{r}\text {-Scroll-case } \\
\text { temperature }\end{array} \\
18.9\end{array}$} & \multirow[t]{2}{*}{$\begin{array}{c}\text { water } \\
\left({ }^{\circ} \mathrm{C}\right)\end{array}$} & \multirow{2}{*}{$\frac{\begin{array}{c}\text { Numbers } \\
\text { detected }\end{array}}{0}$} & \multirow{2}{*}{$\begin{array}{c}\text { Adjusted } \\
\text { numbers detected } \\
0\end{array}$} \\
\hline 17 & Aug & & & & & & \\
\hline 18 & Aug & 38.9 & 0.0 & 20.2 & & 0 & 0 \\
\hline 19 & Aug & 37.9 & 0.0 & 20.4 & & 0 & 0 \\
\hline 20 & Aug & 36.1 & 0.0 & 18.9 & & 0 & 0 \\
\hline 21 & Aug & 34.9 & 0.0 & 18.9 & & 1 & 1 \\
\hline 22 & Aug & 34.8 & 0.0 & 20.3 & & 0 & 0 \\
\hline 23 & Aug & 34.9 & 0.0 & 18.9 & & 0 & 0 \\
\hline 24 & Aug & 36.4 & 0.0 & 18.3 & & 0 & 0 \\
\hline 25 & Aug & 35.0 & 0.0 & 20.2 & . & 0 & 0 \\
\hline 26 & Aug & 34.8 & 0.0 & 19.6 & & 0 & 0 \\
\hline 27 & Aug & 33.9 & 0.0 & 18.3 & & 0 & 0 \\
\hline 28 & Aug & 31.8 & 0.0 & 18.3 & & 1 & 1 \\
\hline 29 & Aug & 34.7 & 0.0 & 18.3 & & 0 & 0 \\
\hline 30 & Aug & 34.5 & 0.0 & 18.3 & & 0 & 0 \\
\hline 31 & Aug & 33.5 & 0.0 & 20.0 & & 0 & 0 \\
\hline 01 & Sep & 31.7 & 0.0 & 20.4 & & 0 & 0 \\
\hline 02 & Sep & 30.3 & 0.0 & 21.2 & & 0 & 0 \\
\hline 03 & Sep & 27.7 & 0.0 & 22.2 & & $Q$ & 0 \\
\hline 04 & Sep & 25.4 & 0.0 & 19.4 & & 0 & 0 \\
\hline 05 & Sep & 24.4 & 0.0 & 19.4 & & 0 & 0 \\
\hline 06 & Sep & 27.1 & 0.0 & 18.3 & & 0 & 0 \\
\hline 07 & Sep & 28.8 & 0.0 & 18.3 & & 1 & 1 \\
\hline 08 & Sep & 28.7 & 0.0 & 18.9 & & 0 & 0 \\
\hline 09 & Sep & 24.6 & 0.0 & 18.9 & & 0 & 0 \\
\hline
\end{tabular}


Appendix Table 25. Daily detections of PIT-tagged wild spring/summer chinook salmon smolts from Idaho at Lower Monumental Dam during 1995, with associated river flows (kcfs), spill (kcfs), and water temperatures ("C) at the dam. Numbers detected represent fish not detected at a previous dam. Adjusted numbers detected are calculated during spill.

\begin{tabular}{|c|c|c|c|c|c|c|c|c|}
\hline \multicolumn{2}{|c|}{ Date } & \multirow{2}{*}{\begin{tabular}{c}
\multicolumn{2}{c}{$\begin{array}{c}\text { Average } \\
\text { flow (kcfs) }\end{array}$} \\
65.8
\end{tabular}} & \multirow{2}{*}{ 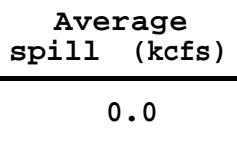 } & \multirow{2}{*}{$\begin{array}{r}\begin{array}{c}\text { Scroll-case } \\
\text { temperature }\end{array} \\
8.9\end{array}$} & \multirow{2}{*}{$\begin{array}{c}\text { water } \\
\left({ }^{\circ} \mathrm{C}\right)\end{array}$} & \multirow{2}{*}{$\frac{\begin{array}{c}\text { Numbers } \\
\text { detected }\end{array}}{0}$} & \multicolumn{2}{|c|}{$\begin{array}{c}\text { Adjusted } \\
\text { numbers detected }\end{array}$} \\
\hline 13 & Apr & & & & & & & 0 \\
\hline 14 & Apr & 78.1 & 2.0 & ---- & & 0 & & 0 \\
\hline 15 & Apr & 78.9 & 5.3 & 8.9 & & 0 & & 0 \\
\hline 16 & Apr & 78.6 & 5.3 & 8.9 & & 0 & & 0 \\
\hline 17 & Apr & 77.3 & 4.9 & ---- & & 0 & & 0 \\
\hline 18 & Apr & 64.7 & 4.9 & 9.4 & & 0 & & 0 \\
\hline 19 & Apr & 62.2 & 6.5 & 9.4 & & 1 & & 1 \\
\hline 20 & Apr & 76.9 & 10.1 & 9.4 & & 0 & & 0 \\
\hline 21 & Apr & 73.5 & 12.0 & 9.4 & & 0 & & 0 \\
\hline 22 & Apr & 63.3 & 13.2 & 9.4 & & 1 & & 1 \\
\hline 23 & Apr & 52.8 & 15.7 & 9.4 & & 0 & & 0 \\
\hline 24 & Apr & 56.1 & 15.3 & 9.4 & & 3 & & 4 \\
\hline 25 & Apr & 63.7 & 15.4 & 9.4 & & 1 & & 1 \\
\hline 26 & Apr & 65.7 & 15.7 & 9.4 & & 3 & - & 4 \\
\hline 27 & Apr & 75.2 & 14.3 & 9.4 & & 1 & & 1 \\
\hline 28 & Apr & 75.5 & 14.0 & 9.4 & & 0 & & 0 \\
\hline 29 & Apr & 82.7 & 13.8 & 9.4 & & 3 & & 4 \\
\hline 30 & Apr & 85.6 & 14.0 & --- & & 1 & & 1 \\
\hline 01 & May & 82.0 & 15.2 & 10.0 & & 7 & & 9 \\
\hline 02 & May & 77.4 & 15.6 & 10.6 & & 2 & 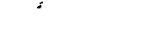 & 3 \\
\hline 03 & May & 95.9 & 17.0 & 10.6 & & 4 & & 5 \\
\hline 04 & May & 100.0 & 18.1 & 10.6 & & 4 & & 5 \\
\hline 05 & May & 101.3 & 19.6 & 10.6 & & 14 & & 17 \\
\hline 06 & May & 98.2 & . 20.2 & 10.6 & & 10 & & 13 \\
\hline 07 & May & 117.0 & 24.6 & 10.6 & & 9 & & 11 \\
\hline 08 & May & 128.1 & 31.6 & 10.6 & & 8 & & 11 \\
\hline 09 & May & 115.5 & 27.9 & 10.6 & & 4 & & 5 \\
\hline 10 & May & 110.0 & 26.2 & 10.6 & & 9 & & 12 \\
\hline 11 & May & 113.5 & 23.8 & 10.6 & & 2 & & 3 \\
\hline 12 & May & 98.8 & 18.1 & 10.6 & & 7 & & 9 \\
\hline
\end{tabular}


Appendix Table 25. Continued.

\begin{tabular}{|c|c|c|c|c|c|c|}
\hline \multicolumn{2}{|c|}{ Date } & \multirow{2}{*}{$\begin{array}{c}\text { Average } \\
\text { flow (kcfs) }\end{array}$} & \multirow{2}{*}{$\begin{array}{c}\text { Average } \\
\text { spill (kcfs) } \\
23.2\end{array}$} & \multirow{2}{*}{$\begin{array}{r}\begin{array}{c}\text { Scroll-case } \\
\text { temperature }\end{array} \\
10.6\end{array}$} & \multirow{2}{*}{$\begin{array}{c}\begin{array}{c}\text { Numbers } \\
\text { detected }\end{array} \\
14\end{array}$} & \multirow{2}{*}{$\begin{array}{c}\text { Adjusted } \\
\text { numbers detected } \\
18\end{array}$} \\
\hline 13 & Ma y & & & & & \\
\hline 14 & May & 102.9 & 17.7 & 10.6 & 12 & 14 \\
\hline 15 & May & 91.9 & 18. 5 & 10.6 & 8 & 10 \\
\hline 16 & Ma y & 103.0 & 1' 9.4 & $-\cdots$ & 7 & 9 \\
\hline 17 & May & 99.6 & 18.6 & 11.1 & 16 & 20 \\
\hline 18 & May & 108. 3 & 19.0 & 11. 7 & 9 & 11 \\
\hline 19 & May & 109.8 & 21. 1 & $-\cdots$ & 4 & 5 \\
\hline 20 & May & 117.7 & 26.4 & $-\cdots$ & 12 & 15 \\
\hline 21 & May & 111.6 & 24.9 & --- & 7 & 9 \\
\hline 22 & May & 117.2 & 25.1 & 13. 3 & 6 & 8 \\
\hline 23 & May & 120.7 & 26.0 & 13. 3 & 5 & 6 \\
\hline 24 & May & 113.9 & 22.9 & 13. 3 & 3 & 4 \\
\hline 25 & May & 115. $0^{\prime}$ & 17.4 & 13. 3 & 6 & 7 \\
\hline 26 & May & 111.1 & 4. 5 & 13. 9 & 4 & 4 \\
\hline 27 & Ma y & 109.3 & 2. 0 & 13.9 & 1 & 1 \\
\hline 28 & May & 108. 3 & 2. 5 & 14.4 & 3 & 3 \\
\hline 29 & May & 98.7 & 15.8 & 14.4 & 1 & 1 \\
\hline 30 & Ma y & 117.1 & 11. 2 & 14.4 & 2 & 2 \\
\hline 31 & May & 117.9 & 10.8 & 14.4 & 4 & 4 \\
\hline 01 & Jun & 131.9 & 26.3 & 14.4 & 1 & 1 \\
\hline 02 & Jun & 132.4 & 34.8 & 14.4 & 3 & 4 \\
\hline 03 & Jun & 134.0 & 27.2 & 14.4 & 1 & 1 \\
\hline 04 & Jun & 139.5 & 33.1 & 14.4 & 1 & 1 \\
\hline 05 & Jun & 145.0 & 37.2 & 15.0 & 5 & 7 \\
\hline 06 & Jun & 148.7 & 41.7 & 15.0 & 1 & 1 \\
\hline 07 & Jun & 143. 1 & 39.2 & $\cdots$ & 3 & 4 \\
\hline 08 & Jun & 132.1 & 24.9 & 15.0 & 3 & 4 \\
\hline 09 & Jun & 123.6 & 16. 5 & 14.4 & 5 & 6 \\
\hline 10 & Jun & 116.3 & 9.4 & 14.4 & 4 & 4 \\
\hline 11 & Jun & 103.6 & 0.0 & 13. 9 & 2 & 2 \\
\hline 12 & Jun & 110.6 & 5.3 & 13.9 & 4 & 4 \\
\hline 13 & Jun & 118. 2 & 14.5 & 13. 9 & 3 & 3 \\
\hline 14 & Jun & 114.0 & 16. 2 & 12.8 & 2 & 2 \\
\hline
\end{tabular}


Appendix Table 25. Continued.

\begin{tabular}{|c|c|c|c|c|c|c|}
\hline Date & \multirow{2}{*}{$\begin{array}{c}\begin{array}{c}\text { Average } \\
\text { flow (kcfs) }\end{array} \\
119.0\end{array}$} & \multirow{2}{*}{$\begin{array}{c}\text { Average } \\
\text { spill (kcfs) } \\
18.7\end{array}$} & \multirow{2}{*}{$\begin{array}{r}\begin{array}{c}\text { Scroll-case } \\
\text { temperature }\end{array} \\
12.8\end{array}$} & \multirow{2}{*}{$\frac{\begin{array}{c}\text { Numbers } \\
\text { detected }\end{array}}{0}$} & \multicolumn{2}{|c|}{$\begin{array}{c}\text { Adjusted } \\
\text { numbers detected }\end{array}$} \\
\hline 15 Jun & & & & & & 0 \\
\hline 16 Jun & 122.7 & 18.9 & 12.8 & 2 & & 2 \\
\hline 17 Jun & 117.1 & 13.7 & 12.8 & 0 & & 0 \\
\hline 18 Jun & 116.8 & 13.0 & 15.6 & 2 & & 2 \\
\hline 19 Jun & 117.9 & 13.8 & 15.6 & 1 & & 1 \\
\hline 20 Jun & 118.2 & 15.6 & 14.4 & 0 & & 0 \\
\hline 21 Jun & 112.3 & 11.5 & 15.6 & 0 & & 0 \\
\hline 22 Jun & 100.1 & 10.2 & 15.6 & 1 & & 1 \\
\hline 23 Jun & 85.9 & 9.5 & 15.6 & 0 & & $0--$ \\
\hline 24 Jun & 89.9 & 9.9 & 15.6 & 4 & & 4 \\
\hline 25 Jun & 89.6 & 9.5 & 15.6 & 0 & & 0 \\
\hline 26 Jun & 89.6 & 11.7 & 15.6 & 0 & & 0 \\
\hline 27 Jun & 90.5 & 14.7 & 15.6 & 1 & & 1 \\
\hline 28 Jun & 99.0 & 8.0 & 15.6 & 1 & & 1 \\
\hline 29 Jun & 103.2 & 0.0 & 15.6 & 1 & & 1 \\
\hline 30 Jun & 96.4 & 0.0 & 16.7 & 0 & & 0 \\
\hline $01 \mathrm{Jul}$ & 90.3 & 0.0 & 16.7 & 1 & & 1 \\
\hline $02 \mathrm{Jul}$ & 82.2 & 0.0 & 17.2 & 2 & & 2 \\
\hline $03 \mathrm{Jul}$ & 74.4 & 0.0 & 17.8 & 1 & & 1 \\
\hline $04 \mathrm{Jul}$ & 92.3 & 0.0 & 17.8 & 1 & & 1 \\
\hline $05 \mathrm{Jul}$ & 93.7 & 0.0 & 19.4 & 0 & & 0 \\
\hline $06 \mathrm{Jul}$ & 76.3 & 0.0 & 18.3 & 0 & & 0 \\
\hline $07 \mathrm{Jul}$ & 70.9 & 0.0 & 18.3 & 0 & & 0 \\
\hline $08 \mathrm{Jul}$ & 77.2 & 0.0 & 18.3 & 0 & & 0 \\
\hline $09 \mathrm{Jul}$ & 69.5 & 0.0 & 18.3 & 1 & & 1 \\
\hline $10 \mathrm{Jul}$ & 78.0 & 0.0 & 18.3 & 1 & & 1 \\
\hline $11 \mathrm{Jul}$ & 74.8 & 0.0 & 18.3 & 0 & & 0 \\
\hline $12 \mathrm{Jul}$ & 76.1 & 0.0 & 18.3 & 0 & & 0 \\
\hline $13 \mathrm{Jul}$ & 74.3 & 0.0 & 18.3 & 0 & & 0 \\
\hline $14 \mathrm{Jul}$ & 68.9 & 0.0 & 18.3 & 0 & & 0 \\
\hline $15 \mathrm{Jul}$ & 58.3 & 0.0 & 19.4 & 0 & & 0 \\
\hline $16 \mathrm{Jul}$ & 57.7 & 0.0 & 19.4 & 0 & & 0 \\
\hline $17 \mathrm{Jul}$ & 52.7 & 0.0 & 19.4 & 0 & & 0 \\
\hline
\end{tabular}


Appendix Table 25. Continued.

\begin{tabular}{|c|c|c|c|c|c|c|}
\hline \multicolumn{2}{|c|}{ Date } & \multirow{2}{*}{$\begin{array}{c}\text { Average } \\
\text { flow (kCfs) }\end{array}$} & $\begin{array}{c}\text { Average } \\
\text { spill } \quad \text { (kcfs) }\end{array}$ & \multirow{2}{*}{$\begin{array}{r}\begin{array}{r}\text { Scroll-case } \\
\text { temperature }\end{array} \\
20.0\end{array}$} & \multirow{2}{*}{$\frac{\begin{array}{c}\text { Numbers } \\
\text { detected }\end{array}}{0}$} & \multirow{2}{*}{\begin{tabular}{c}
\multicolumn{2}{c}{ Adjusted } \\
numbers detected \\
0
\end{tabular}} \\
\hline 18 & Jul & & 0.0 & & & \\
\hline 19 & Jul & 53.7 & 0.0 & 21.1 & 0 & 0 \\
\hline 20 & Jul & 52.1 & 0.0 & 21.1 & $0^{\prime}$ & 0 \\
\hline 21 & Jul & 50.6 & 0.0 & 21.1 & 0 & 0 \\
\hline 22 & Jul & 49.2 & 0.0 & 21.1 & 0 & 0 \\
\hline 23 & Jul & 47.8 & 0.0 & 21.1 & 0 & 0 \\
\hline 24 & Jul & 46. 2 & 0.0 & 21.1 & 0 & 0 \\
\hline 25 & Jul & 51.6 & 0.0 & 21.1 & 0 & 0 \\
\hline 26 & Jul & 52.2 & 0.0 & 22.2 & 0 & 0 \\
\hline 27 & Jul & 51.9 & 0.0 & 21.1 & 1 & 1 \\
\hline 28 & Jul & 49.4 & 0.0 & 21.1 & 0 & 0 \\
\hline 29 & Jul & 52.4 & 0.0 & 21.1 & 0 & 0 \\
\hline 30 & Jul & 47.6 & 0.0 & 21.1 & 0 & 0 \\
\hline 31 & Jul & 46. 7 & 0.0 & 21. 1 & 0 & 0 \\
\hline 01 & Aug & 47. 1 & 0.0 & 22. 5. & 0 & 0 \\
\hline 02 & Aug & 48. 6 & 0.0 & 22.6 & 0 & 0 \\
\hline 03 & Aug & 45. 0 & 0.0 & 22.4 & 0 & 0 \\
\hline 04 & Aug & 45.9 & 0.0 & 21.9 & 0 & 0 \\
\hline 05 & Aug & 46. 5 & 0.0 & 21.9 & 0 & 0 \\
\hline 06 & Aug & 40. 7 & 0.0 & 22.1 & 1 & 1 \\
\hline 07 & Aug & 46. 5 & 0.0 & 21.8 & 0 & 0 \\
\hline 08 & Aug & 43. 5 & 0.0 & 21.7 & 0 & 0 \\
\hline 09 & Aug & 41.5 & 0.0 & 21.6 & 1 & 1 \\
\hline 10 & Aug & 43. 0 & 0.0 & 21.9 & 0 & 0 \\
\hline 11 & Aug & 34.3 & 0.0 & 21.4 & 0 & 0 \\
\hline 12 & Aug & 36.7 & 0.0 & 21.4 & 0 & 0 \\
\hline 13 & Aug & 33.9 & 0.0 & 22. 2 & 0 & 0 \\
\hline 14 & Aug & 34.4 & 0.0 & 22.5 & 0 & 0 \\
\hline 15 & Aug & 35.3 & 0.0 & 22.5 & 0 & 0 \\
\hline 16 & Aug & 35.8 & 0.0 & 24.0 & 0 & 0 \\
\hline 17 & Aug & 35.0 & 0.0 & 24.0 & 0 & 0 \\
\hline 18 & Aug & 39.9 & 0.0 & 23.0 & 0 & 0 \\
\hline 19 & Aug & 38.8 & 0.0 & 22. 2 & 0 & 0 \\
\hline
\end{tabular}


Appendix Table 25. Continued.

\begin{tabular}{ccccccc}
\hline Date & $\begin{array}{c}\text { Average } \\
\text { flow }\end{array}$ (kcfs) & $\begin{array}{c}\text { Average } \\
\text { spill }\end{array}$ (kcfs) & $\begin{array}{c}\text { Scroll-case } \\
\text { temperature }\end{array}$ & $\begin{array}{c}\text { water } \\
\left({ }^{\circ} \mathrm{C}\right)\end{array}$ & $\begin{array}{c}\text { Numbers } \\
\text { detected }\end{array}$ & $\begin{array}{c}\text { Adjusted } \\
\text { numbers }\end{array}$ \\
\hline 20 Aug & 36.7 & 0.0 & 22.6 & 0 & 0 \\
21 Aug & 34.7 & 0.0 & 23.1 & 0 & 0 \\
22 Aug & 35.8 & 0.0 & 21.6 & 0 & 0 \\
23 Aug & 35.1 & 0.0 & 21.0 & 0 & 0 \\
24 Aug & 36.1 & 0.0 & 20.5 & 0 & 0 \\
25 Aug & 34.8 & 0.0 & 20.7 & 0 & 0 \\
26 Aug & 35.7 & 0.0 & 20.5 & 0 & 0 \\
27 Aug & 33.9 & 0.0 & 20.8 & 1 & 0 \\
28 Aug & 32.4 & 0.0 & 19.4 & 0 & 0 \\
29 Aug & 34.2 & 0.0 & 19.4 & 0 & 0 \\
30 Aug & 35.0 & 0.0 & 19.4 & 0 & 0 \\
31 Aug & 33.1 & 0.0 & 19.4 & 0 & 0 \\
\hline
\end{tabular}


Appendix Table 26. Daily detections of PIT-tagged wild spring/summer chinook salmon smolts from Idaho at McNary Dam during 1995, with associated river flows (kcfs), spill (kcfs), and water temperatures $\left({ }^{\circ} \mathrm{C}\right)$ at the dam. Numbers detected represent fish not detected at a previous dam. Adjusted numbers detected are calculated during spill.

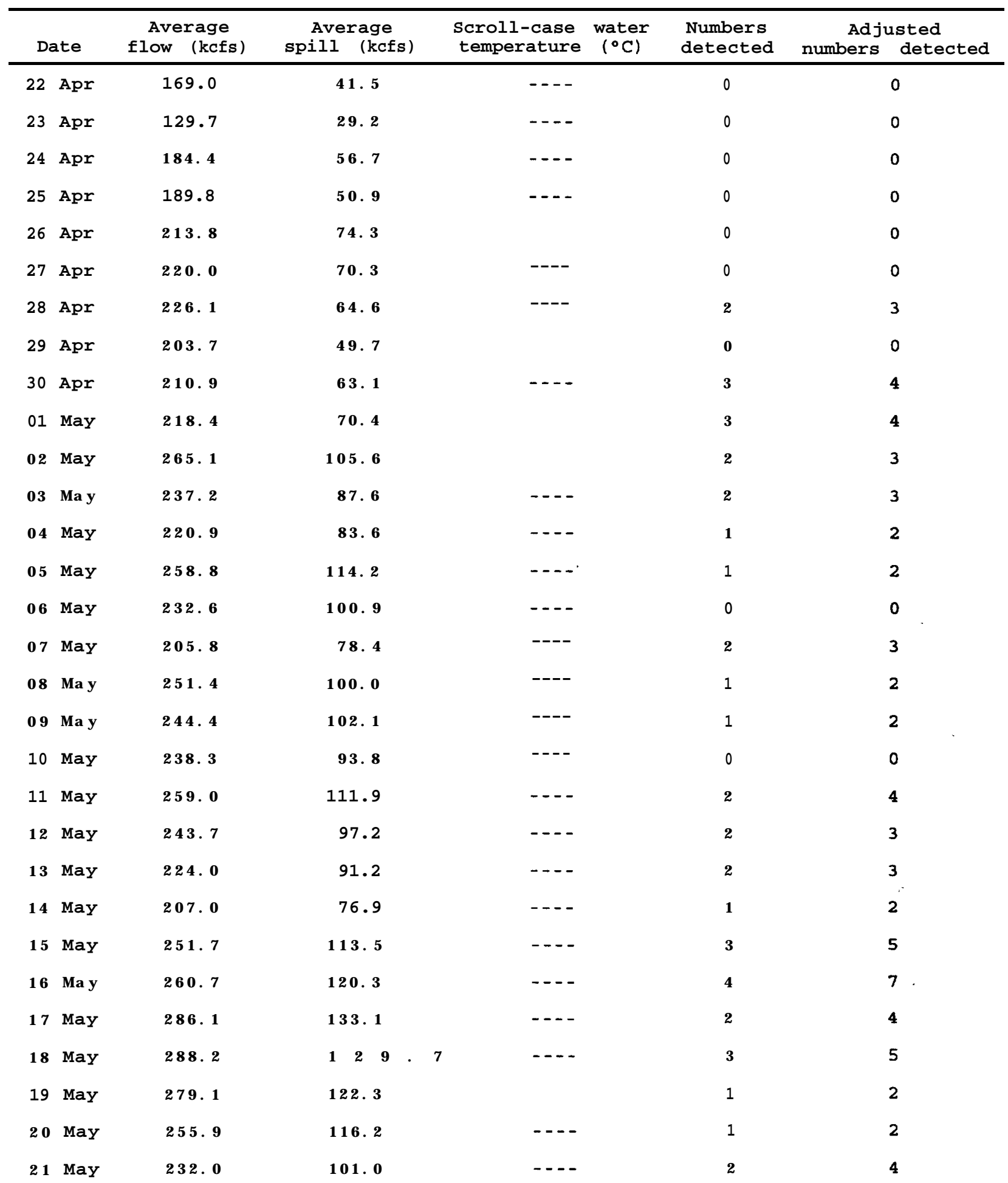


Appendix Table 26. Continued.

\begin{tabular}{|c|c|c|c|c|c|c|c|}
\hline \multicolumn{2}{|c|}{ Date } & \multirow{2}{*}{\begin{tabular}{c}
\multicolumn{2}{c}{$\begin{array}{c}\text { Average } \\
\text { flow (kcfs) }\end{array}$} \\
259.8
\end{tabular}} & \multirow{2}{*}{$\begin{array}{c}\begin{array}{c}\text { Average } \\
\text { spill (kcfs) }\end{array} \\
114.9\end{array}$} & \multicolumn{2}{|c|}{$\begin{array}{c}\text { Scroll-case water } \\
\text { temperature }\left({ }^{\circ} \mathrm{C}\right)\end{array}$} & \multirow{2}{*}{$\begin{array}{c}\begin{array}{c}\text { Numbers } \\
\text { detected }\end{array} \\
1\end{array}$} & \multirow{2}{*}{\begin{tabular}{c}
\multicolumn{2}{c}{ Adjusted } \\
numbers detected \\
2
\end{tabular}} \\
\hline 22 & May & & & 12.8 & & & \\
\hline 23 & May & 272. 3 & 127.1 & 12.8 & & 5 & 9 \\
\hline 24 & May & 265. 1 & 119.0 & 12. 8 & & 0 & 0 \\
\hline 25 & May & 277.1 & 133. 3 & 12. 8 & & 0 & ${ }^{0}$ \\
\hline 26 & May & 269.6 & 121.8 & 13.9 & & 1 & 2 \\
\hline 27 & Ma y & 247.3 & 101.9 & 12. 8 & & 0 & 0 \\
\hline 28 & Ma y & 213.5 & 78.6 & 13. 9 & & 1 & 2 \\
\hline 29 & May & 228.6 & 92.5 & 14.4 & & 0 & 0 \\
\hline 30 & Ma y & 282. 8 & 129.7 & 14.4 & & 2 & 4 \\
\hline 31 & May & 309.7 & 119.9 & 14.4 & & 0 & 0 \\
\hline 01 & Jun & 292.4 & 118.1 & 14. 4 & & 2 & 3 \\
\hline 02 & Jun & 286.4 & 109.3 & 14.4 & & 0 & 0 \\
\hline 03 & Jun & 264.9 & 110.2 & 15.0 & & 3 & 5 \\
\hline 04 & Jun & $2 i \mathrm{i} 0.0$ & 107. 3 & 15. 0 & & 1 & 2 \\
\hline 05 & Jun & 276.3 & 117.0 & 15.0 & & 0 & 0 \\
\hline 06 & Jun & 285.5 & 123.7 & 15.0 & & 3 & 5 \\
\hline 07 & Jun & 260.0 & 135.1 & 15.0 & & 0 & 0 \\
\hline 08 & Jun & 274.5 & 12711 & 14.4 & & 0 & 0 \\
\hline 09 & Jun & 270.0 & 118.8 & 14.4 & & 0 & 0 \\
\hline 10 & Jun & 280.6 & 125.1 & 14. 4 & & 0 & 0 \\
\hline 11 & Jun & 277.9 & 140.7 & 14.4 & & 0 & 0 \\
\hline 12 & Jun & 290.1 & 134.8 & 15.0 & & 1 & 2 \\
\hline 13 & Jun & 289.6 & 131.6 & 15. 0 & & 0 & 0 \\
\hline 14 & Jun & 295.6 & 137.3 & $15 \cdot 0$ & & 0 & 0 \\
\hline 15 & Jun & 298.1 & 134.6 & 15. 0 & & 1 & 2 \\
\hline 16 & Jun & 274.6 & 109. 3 & 15.0. & & 0 & 0 \\
\hline 17 & Jun & 253. 1 & 92.2 & 15.0 & & 1 & 2 \\
\hline 18 & Jun & 247.6 & 95.5 & 15. 0 & & 0 & 0 \\
\hline 19 & Jun & 254.6 & 105.0 & $15 \cdot 0$ & & 0 & 0 \\
\hline 20 & Jun & 286.0 & 124.7 & 13.9 & & 0 & 0 \\
\hline 21 & Jun & 279.6 & 109.9 & 15.0 & & 0 & 0 \\
\hline 22 & Jun & 285.6 & 116.3 & 15.0 & & 0 & 0 \\
\hline 23 & Jun & 277.0 & 122. 2 & 15.0 & & 0 & 0 \\
\hline
\end{tabular}


Appendix Table 26. Continued.

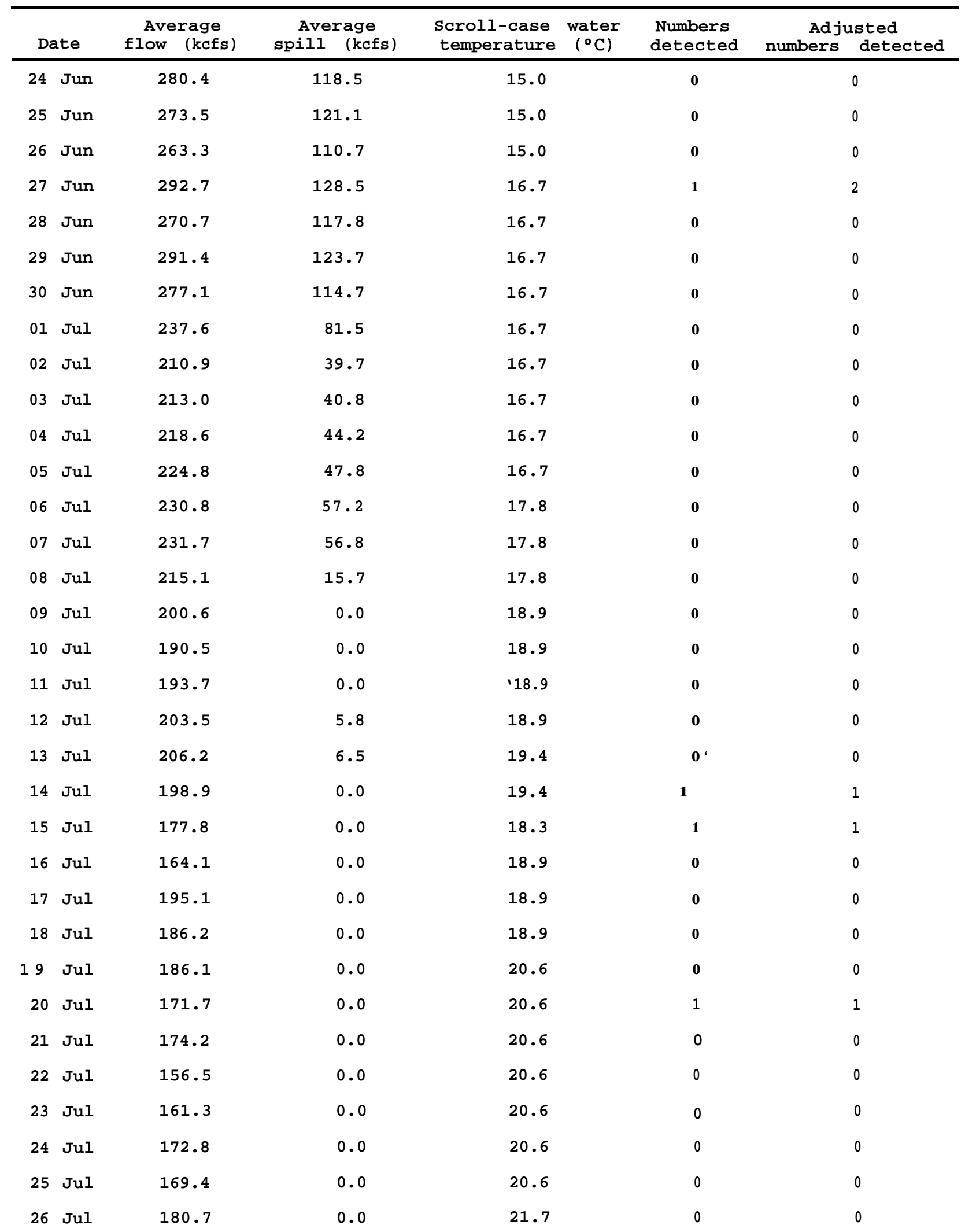


Appendix Table 26. Continued.

\begin{tabular}{|c|c|c|c|c|c|c|}
\hline Date & $\begin{array}{l}\text { Average } \\
\text { flow (kcfs) }\end{array}$ & $\begin{array}{c}\text { Average } \\
\text { spill } \quad \text { (kcfs) }\end{array}$ & $\begin{array}{l}\text { Scroll-case } \\
\text { temperature }\end{array}$ & $\begin{array}{l}\text { water } \\
\left({ }^{\circ} \mathrm{C}\right)\end{array}$ & $\begin{array}{l}\text { Numbers } \\
\text { detected }\end{array}$ & $\begin{array}{c}\text { Adjusted } \\
\text { numbers detected }\end{array}$ \\
\hline $27 \mathrm{Jul}$ & 169.5 & 5.2 & 20.6 & & 0 & 0 \\
\hline $28 \mathrm{Jul}$ & 206.9 & 19.9 & 20.6 & & 0 & 0 \\
\hline $29 \mathrm{Jul}$ & 165.8 & 0.0 & 20.6 & & 0 & 0 \\
\hline $30 \mathrm{Jul}$ & $164 . i$ & 0.0 & 20.6 & & 0 & 0 \\
\hline $31 \mathrm{Jul}$ & 164.5 & 0.0 & 20.6 & & 0 & 0 \\
\hline
\end{tabular}


Appendix Table 27. Minimum, maximum, and average depth (in feet) by month at five monitoring sites in the Salmon River drainage from August 1994 through July 1995. These data were provided by Pacific Northwest Laboratories.

Marsh Creek (RKm 179.5 from mouth of the Middle Fork Salmon River)

\begin{tabular}{|c|c|c|c|c|c|c|c|c|c|c|c|c|}
\hline & August & September & October & November & December & January & February & March & April & May & June & July \\
\hline Average & 0.76 & 0.64 & 1.05 & 1.23 & 1.58 & 1.53 & 1.07 & 0.74 & 1.04 & 2.35 & 3.34 & 2.84 \\
\hline Minimum & 0.40 & 0.30 & 0.65 & 0.60 & 0.83 & 0.88 & 0.40 & 0.37 & 0.75 & 1.20 & 3.05 & 2.30 \\
\hline Maximum & 1.10 & 0.90 & 1.33 & 2.17 & 2.34 & 2.46 & 1.51 & 1.11 & 1.38 & 3.47 & 3.79 & 3.40 \\
\hline
\end{tabular}

\section{Middle Fork Salmon River near Thomas Creek (RKm 97.6)}

\begin{tabular}{|c|c|c|c|c|c|c|c|c|c|c|c|c|}
\hline & August' & September & October & November & December & January & February & March & April & May $^{\mathbf{a}}$ & June' & July" \\
\hline Average & ---- & 1.28 & 1.28 & 1.18 & 1.35 & 1.51 & 1.90 & 2.12 & 2.79 & ---- & ---- & $i^{---}$ \\
\hline Minimum & ---- & 1.00 & 0.86 & -0.86 & 1.03 & 1.26 & 1.01 & 1.40 & 2.16 & - & - & - \\
\hline Maximum & ---- & 1.60 & 1.90 & 1.46 & 1.72 & 1.70 & 2.71 & 2.87 & 3.33 & ---- & ---- & ---- \\
\hline
\end{tabular}

Salmon River near Sawtooth Hatcher-v (RKrn 627.9)

\begin{tabular}{|c|c|c|c|c|c|c|c|c|c|c|c|c|}
\hline & August & September & October & November & December & January & February & March & April & May & June & July \\
\hline Average & 1.00 & 0.98 & 1.26 & 2.66 & 2.81 & 2.85 & 2.89 & 2.86 & 3.07 & 3.63 & 4.49 & 4.55 \\
\hline Minimum & 0.70 & 0.70 & 1.10 & 2.14 & 2.46 & 2.45 & 2.21 & 2.47 & 2.71 & 3.09 & 4.18 & 4.05 \\
\hline Maximum & 1.30 & 1.30 & 1.45 & 3.18 & 3.12 & 3.33 & 3.35 & 3.30 & 3.33 & 4.34 & 5.05 & 4.97 \\
\hline
\end{tabular}


Appendix Table 27. Continued.

Valley Creek (RKm 609.4 from the mouth of the Salmon River)

\begin{tabular}{|c|c|c|c|c|c|c|c|c|c|c|c|c|}
\hline & August & September & October & November & December & January & February & March & April & May & June & July \\
\hline Average & 0.54 & 0.46 & $-\ldots$ & 1.97 & 2. 04 & 2.05 & 2. 17 & 1. 81 & 2. 25 & 3. 06 & 3. 82 & 3. 41 \\
\hline Minimum & 0.20 & 0.10 & $\ldots$ & 1. 57 & 1. 70 & 1.70 & 1.54 & 1. 37 & 1. 91 & 2. 35 & 3. 45 & 2. 80 \\
\hline Maximum & 0.90 & 0.70 & -- & 2.41 & 2.32 & 2. 42 & 2. 73 & 2. 25 & 2.65 & 3. 83 & 4. 24 & 4. 03 \\
\hline
\end{tabular}

$\underline{\text { Salmon River below confluence with Yankee Fork (RKm 590.7) }}$

\begin{tabular}{|c|c|c|c|c|c|c|c|c|c|c|c|c|}
\hline & August & September & October & November & December & January & February & March & April & May & June & July \\
\hline Average & 0.36 & 0.20 & 1.61 & 2.40 & 2.51 & $\ldots$ & 2.15 & 2.31. & 3.06 & 4. 93 & 6.98 & 5. 59 \\
\hline Minimum & 0.00 & 0.10 & 1.57 & 1.97 & 2.29 & ---- & 2.15 & 1.87 & 2. 26 & 3. 41 & 6.08 & 3. 96 \\
\hline Maxi mum & 0.80 & 0.50 & 1. 67 & 2. 79 & 2. 75 & $\ldots$ & 2. 15 & 2. 79 & 3. 5 ; & 6.51 & 7.90 & 7. 07 \\
\hline
\end{tabular}

'Data loss occurred when high flows washed out the monitor. 
Appendix Table 28. Minimum, maximum, and average water temperatures $\left({ }^{\circ} \mathrm{C}\right)$ by month at five monitoring sites in the Salmon River drainage from August 1994 through July 1995. These data were provided by Pacific Northwest Laboratories.

Marsh Creek (RKm 179.5 from the mouth of the Middle Fork Salmon River)

\begin{tabular}{|c|c|c|c|c|c|c|c|c|c|c|c|c|}
\hline & August & September & October & November & December & January & February & March & April & May & June & July \\
\hline Average & 11.91 & 8.88 & 4.81 & 0.73 & 0.59 & 0.63 & 1.77 & 1.98 & 3.42 & 4.71 & 7.48 & 10.65 \\
\hline Minimum & 5.40 & 3.10 & 0.20 & 0.10 & 0.30 & 0.30 & 0.20 & 0.10 & 0.20 & 0.40 & 1.40 & 4.90 \\
\hline Maximum & 20.10 & 15.30 & 11.60 & 4.10 & 3.40 & 3.40 & 6.90 & 8.60 & 10.10 & 12.90 & 14.70 & 18.10 \\
\hline
\end{tabular}

$\underline{\text { Middle Fork Salmon River near Thomas Creek (RKm 97.6) }}$

\begin{tabular}{|c|c|c|c|c|c|c|c|c|c|c|c|c|}
\hline & Augusta & September & October & November & December & January & February & March & April & May" & June' & July" \\
\hline Average & ---- & 11.57 & 6.48 & 0.95 & 0.23 & 0.22 & 1.34 & 3.08 & 5.23 & ----- & --- & ---- \\
\hline Minimum & ----- & 8.90 & 1.30 & 0.20 & 0.20 & 0.20 & 0.20 & 0.20 & 2.00 & ----- & ----- & ----- \\
\hline Maximum & ----- & 14.20 & 12.60 & 4.50 & 0.60 & 0.60 & 4.30 & 7.00 & 7.80 & ----- & ---- & ---- \\
\hline
\end{tabular}

$\underline{\text { Salmon River near Sawtooth Hatchery (RKm 627.9) }}$

\begin{tabular}{|c|c|c|c|c|c|c|c|c|c|c|c|c|}
\hline & August & September & October & November & December & January & February & March & April & May & June & July \\
\hline Average & 13.95 & 11.40 & 8.70 & 1.29 & 1.48 & 1.45 & 2.81 & 3.56 & 6.16 & 7.85 & 9.70 & 13.24 \\
\hline Minimum & 8.40 & 6.60 & 4.50 & 0.10 & 0.10 & 0.10 & 0.10 & 0.30 & 1.00 & 3.30 & 4.10 & 8.40 \\
\hline Maximum & 20.80 & 17.20 & 17.10 & 5.30 & 4.60 & 4.90 & 7.60 & 9.80 & 11.50 & 13.10 & 15.90 & 19.00 \\
\hline
\end{tabular}


Appendix Table 28. Continued.

Valley Creek (RKm 627.9 from the mouth of the Salmon River)

\begin{tabular}{|c|c|c|c|c|c|c|c|c|c|c|c|c|}
\hline & August & September & October & November & December & January & February & $\mathrm{March}$ & April & May & June & July \\
\hline Average & 15.26 & 11.56 & ------ & 0.74 & 0.76 & 0.74 & 1.41 & 2.61 & 4.59 & 7.66 & 9.74 & 13.21 \\
\hline Minimum & 8.10 & 4.90 & ---- & 0.30 & 0.30 & 0.20 & 0.20 & $0.30^{\prime}$ & 0.30 & 1.60 & 3.50 & 7.90 \\
\hline Maximum & 23.40 & 18.90 & ----- & 1.70 & 1.40 & 1.50 & 6.40 & 9.60 & 11.30 & 14.30 & 16.10 & 20.40 \\
\hline
\end{tabular}

$\underline{\text { Salmon River below confluence with Yankee Fork (RKm 590.7) }}$

\begin{tabular}{|c|c|c|c|c|c|c|c|c|c|c|c|c|}
\hline & August & September & October & November & December & January & February & March & April & May & June & July \\
\hline Average & 14.11 & 13.32 & 9.51 & 0.39 & 0.63 & ----- & 1.40 & 2.74 & 5.12 & 6.53 & 7.95 & 12.77 \\
\hline Minimum & 9.10 & 9.10 & 6.50 & 0.20 & 0.20 & ----- & 0.40 & 0.20 & 0.80 & 2.60 & 3.70 & 7.50 \\
\hline Maximum & 18.60 & 17.30 & 12.40 & 1.20 & 2.30 & ----- & 2.20 & 7.20 & 9.60 & 11.10 & 12.90 & 18.00 \\
\hline
\end{tabular}

"Data loss occurred when high flows washed out the monitor. 
Appendix Table 29. Minimum, maximum, and average $\mathrm{pH}$ by month at five monitoring sites in the Salmon River drainage from August 1994 through July 1995. These data were provided by Pacific Northwest Laboratories.

Marsh Creek (RKm 179.5 from the mouth of the Middle Fork Salmon River)

\begin{tabular}{|c|c|c|c|c|c|c|c|c|c|c|c|c|}
\hline & August & September & October & November & December & January & February & March & April & May & June & July \\
\hline Average & 8.00 & 8.13 & 8.24 & 7.92 & 7.86 & 8.06 & 8.54 & 7.75 & 7.59 & 8.22 & 9.37 & 8.19 \\
\hline Minimum & 7.62 & 7.84 & 7.96 & 7.38 & 7.57 & 7.67 & 7.65 & 7.54 & 7.34 & 7.29 & 7.71 & 7.07 \\
\hline Maximum & 8.57 & 8.79 & 9.03 & 9.22 & 8.35 & 8.89 & 9.85 & 8.39 & 8.34 & 10.09 & 10.26 & 9.73 \\
\hline
\end{tabular}

$\underline{\text { Middle Fork Salmon River near Thomas Creek (RKm 97.6) }}$

\begin{tabular}{|c|c|c|c|c|c|c|c|c|c|c|c|c|}
\hline & August" & September & October & November & December & January & February & March & April & May & June' & July \\
\hline Average & ----- & 7.56 & 7.93 & 7.99 & 7.98 & 8.06 & 8.34 & 8.57 & 8.67 & $\overline{-----}$ & $\overline{-----}$ & $\overline{-----}$ \\
\hline Minimum & ----- & 7.16 & 7.53 & 7.79 & 7.78 & 7.89 & 7.86 & 8.31 & 8.38 & ---- & ---- & ----- \\
\hline Maximum & ----- & 7.90 & 8.23 & 8.37 & 8.60 & 8.80 & 9.50 & 9.41 & 9.50 & ----- & ---- & ---- \\
\hline
\end{tabular}

Salmon River near Sawtooth Hatchery (RKm 627.9)

\begin{tabular}{|c|c|c|c|c|c|c|c|c|c|c|c|c|}
\hline & August & September & October & November & December & January & February & March & April & May & June & July \\
\hline Average & 7.70 & 7.83 & 7.88 & 8.21 & 8.29 & 8.42 . & 8.60 & 8.29 & 8.31 & 8.14 & 7.96 & 7.87 \\
\hline Minimum & 7.30 & 7.53 & 7.62 & 7.79 & 7.98 & 8.14 & 8.22 & 7.97 & 7.97 & 7.77 & 7.64 & 7.38 \\
\hline Maximum & 8.30 & 8.28 & 8.30 & 8.97 & 8.80 & 8.91 & 9.35 & 8.73 & 8.98 & 8.80 & 8.66 & 8.62 \\
\hline
\end{tabular}


Appendix Table 29. Continued.

Valley Creek (RKm 627.9 from the mouth of the Salmon River)

\begin{tabular}{|c|c|c|c|c|c|c|c|c|c|c|c|c|}
\hline & August & September & October & November & December & January & February & March & April & May & June & July \\
\hline Average & 8.27 & 8.48 & ----- & 7.48 & 7.53 & 7.50 & 7.73 & 7.71 & 7.56 & 7.49 & 7.49 & 7.47 \\
\hline Minimum & 7.74 & 7.96 & ---- & 7.28 & 7.19 & 6.99 & 7.16 & 7.31 & 7.16 & 7.13 & 7.02 & 6.76 \\
\hline Maximum & 8.87 & 9.12 & ----- & 7.85 & 8.60 & 8.87 & 8.85 & 8.72 & 8.61 & 8.12 & 8.38 & 8.55 \\
\hline
\end{tabular}

$\underline{\text { Salmon River below confluence with Yankee Fork (RKm 590.7) }}$

\begin{tabular}{|c|c|c|c|c|c|c|c|c|c|c|c|c|}
\hline & August & September & October & November & December & January & February & March & April & May & June & July \\
\hline Average & 8.77 & 8.85 & 8.88 & 8.09 & 8.14 & ----- & 7.76 & 7.77 & 7.82 & 7.85 & 7.82 & 7.94 \\
\hline Minimum & 8.24 & 8.38 & 8.53 & 8.00 & 8.02 & ----- & 7.64 & 7.57 & 7.58 & 7.64 & 7.66 & 7.65 \\
\hline Maximum & 9.42 & 9.42 & 9.40 & 8.23 & 8.60 & ----- & 8.08 & 8.19 & 8.25 & 8.26 & 8.07 & 8.70 \\
\hline
\end{tabular}

'Data loss occurred when high flows washed out the monitor. 
Appendix Table 30. Minimum, maximum, and average specific conductance $\left(\mu \mathrm{S} / \mathrm{cm}^{3}\right)$ by month at five monitoring sites in the Snake River drainage from August 1994 through July 1995. These data were provided by Pacific Northwest Laboratories.

Marsh Creek (RKm 179.5 from the mouth of the Middle Fork Salmon River)

\begin{tabular}{|c|c|c|c|c|c|c|c|c|c|c|c|c|}
\hline & August & September & October & November & December & January & February & March & April & May & June & July \\
\hline Average & 66.10 & 68.50 & 71.95 & 60.17 & 62.31 & 65.77 & 63.09 & 50.60 & 47.97 & 36.79 & 32.85 & 38.97 \\
\hline Minimum & 61.00 & 65.00 & 66.00 & 45.00 & 47.00 & 58.00 & 39.00 & 33.00 & 37.00 & 27.00 & 24.00 & 27.00 \\
\hline Maximum & 71.00 & 74.00 & 76.00 & 75.00 & 72.00 & 72.00 & 69.00 & 60.00 & 57.00 & 45.00 & 39.00 & 69.00 \\
\hline
\end{tabular}

Middle Fork Salmon River near Thomas Creek (RKm 97.6)

\begin{tabular}{|c|c|c|c|c|c|c|c|c|c|c|c|c|}
\hline & August $^{a}$ & September & October & November & December & January & February & March & April & May $^{\mathbf{a}}$ & June" & July" \\
\hline Average & ----- & 97.13 & 95.12 & 90.50 & 88.84 & 94.27 & 89.70 & 92.01 & 84.76 & ----- & ----- & ----- \\
\hline Minimum & ----- & 95.00 & 85.00 & 73.00 & 77.00 & 83.00 & 76.00 & 80.00 & 74.00 & ----- & ----- & ----- \\
\hline Maximum & ----- & 98.00 & 98.00 & 103.00 & 106.00 & 111.00 & 99.00 & 104.00 & 97.00 & ----- & ----- & ----- \\
\hline
\end{tabular}

Salmon River near Sawtooth Hatchery (RKm 627.9)

\begin{tabular}{|c|c|c|c|c|c|c|c|c|c|c|c|c|}
\hline & August & September & October & November & December & January & February & March & April & May & June & July \\
\hline Average & 163.17 & 175.87 & 182.96 & 137.73 & 135.36 & 140.91 & 138.76 & 145.25 & 135.34 & 100.73 & 78.62 & 70.63 \\
\hline Minimum & 152.00 & 168.00 & 179.00 & 124.00 & 110.00 & 121.00 & 129.00 & 132.00 & 121.00 & 76.00 & 64.00 & 58.00 \\
\hline Maximum & 173.00 & 186.00 & 188.00 & 149.00 & 154.00 & 158.00 & 157.00 & 161.00 & 157.00 & 125.00 & 97.00 & 88.00 \\
\hline
\end{tabular}


Appendix Table 3 0. Continued.

Valley Creek (RKm 627.9 from the mouth of the Salmon River)

\begin{tabular}{|c|c|c|c|c|c|c|c|c|c|c|c|c|}
\hline & August & September & October & November & December & January & February & March & April & May & June & July \\
\hline Average & 84.01 & 91.66 & ----- & 69.23 & 71.26 & 74.82 & 74.03 & 64.30 & 53.18 & 42.64 & 29.15 & 32.72 \\
\hline Minimum & 76.00 & 85.00 & ----- & 64.00 & 64.00 & 67.00 & 64.00 & 55.00 & 43.00 & 35.00 & 21.00 & 21.00 \\
\hline Maximum & 93.00 & 104.00 & ----- & 79.00 & 80.00 & 82.00 & 86.00 & 179.00 & 68.00 & 51.00 & 40.00 & 45.00 \\
\hline
\end{tabular}

$\underline{\text { Salmon River below confluence with Yankee Fork RKm 590.7) }}$

\begin{tabular}{|c|c|c|c|c|c|c|c|c|c|c|c|c|}
\hline & August & September & October & November & December & January & February & March & April & May & June & July \\
\hline Average & 146.20 & 163.11 & 166.53 & 106.69 & 103.60 & ----- & 94.73 & 87.56 & 80.61 & 63.71 & 47.79 & 54.70 \\
\hline Minimum & 116.00 & 153.00 & 160.00 & 97.00 & 94.00 & ----- & 91.00 & 61.00 & 73.00 & 48.00 & 41.00 & 45.00 \\
\hline Maximum & 165.00 & 173.00 & 176.00 & 128.00 & 119.00 & ----- & 101.00 & 101.00 & 93.00 & 96.00 & 58.00 & 70.00 \\
\hline
\end{tabular}

a Data loss occurred when high flows washed out the monitor. 
Appendix Table 3 1. Minimum, maximum, and average dissolved oxygen (percent -saturation) by month at five monitoring sites in the Salmon River drainage from August 1994 through July 1995. These data were provided by Pacific Northwest Laboratories.

Marsh Creek (RKm 179.5 from the mouth of the Middle Fork Salmon River)

\begin{tabular}{|c|c|c|c|c|c|c|c|c|c|c|c|c|}
\hline & August & September & October & November & December & January & February & March & April & May $^{\mathrm{a}}$ & June' & July $y^{a}$ \\
\hline Average & 85.75 & 88.95 & 90.60 & 90.23 & 100.36 & 90.08 & 52.86 & 72.26 & 81.62 & ----- & ----- & \\
\hline Minimum & 77.38 & 81.41 & 84.84 & 81.70 & 92.05 & 74.42 & 32.82 & 63.50 & 75.20 & ----- & ----- & \\
\hline Maximum & 95.78 & 99.69 & 99.93 & 106.00 & 106.45 & 103.64 & 78.28 & 81.90 & 92.30 & ----- & ----- & \\
\hline
\end{tabular}

$\underline{\text { Middle Fork Salmon River near Thomas Creek (RKm 97.6) }}$

\begin{tabular}{|c|c|c|c|c|c|c|c|c|c|c|c|c|}
\hline & August ${ }^{b}$ & September & October & November & December & January & February & March & April & Mayb & June $^{b}$ & July" \\
\hline Average & ----- & 83.30 & 64.35 & 73.83 & 96.62 & 100.10 & 102.49 & 101.78 & 98.98 & ---- & ---- & ----- \\
\hline Minimum & ----- & 76.00 & 52.80 & 50.50 & 92.40 & 96.90 & 99.90 & 95.30 & 95.00 & ----- & ---- & ---- \\
\hline Maximum & ----- & 93.70 & 86.70 & 95.80 & 103.60 & 104.50 & 107.20 & 106.70 & 105.50 & ----- & ---- & ----- \\
\hline
\end{tabular}

Salmon River near Sawtooth Hatchery (RKm 627.9)

\begin{tabular}{|c|c|c|c|c|c|c|c|c|c|c|c|c|}
\hline & August* & September' & October & November & December & January & February & March & April & May & June & July \\
\hline Average & ------ & ----- & 105.11 & 79.02 & 86.94 & 91.76 & 95.08 & 93.81 & 98.58 & 103.42 & $1 \mathrm{~d} 5.88$ & 93.52 \\
\hline Minimum & ------ & ------ & 93.50 & 73.36 & 79.33 & 86.53 & 88.00 & 86.10 & 89.20 & 86.30 & 84.00 & 74.70 \\
\hline Maximum & ------ & ------ & 142.30 & 88.11 & 97.93 & 102.15 & 113.03 & 109.10 & 121.20 & 121.00 & 122.80 & 120.70 \\
\hline
\end{tabular}


Appendix Table 3 1. Continued.

Valley Creek (RKm 627.9 from the mouth of the Salmon River)

\begin{tabular}{rrccccccccccc}
\hline & August & September & October & November & December & January & February & March & April & May & June & July \\
\hline Average & 92.80 & 83.19 & ----- & 87.41 & 91.54 & 91.09 & 94.19 & 86.36 & 87.50 & 88.25 & 90.37 & 86.31 \\
Minimum & 73.40 & 52.50 & ---- & 83.46 & 87.23 & 82.93 & 70.29 & 81.40 & 82.40 & 83.10 & 79.80 & 73.70 \\
Maximum & 112.60 & 98.00 & ----- & 92.84 & 98.64 & 98.72 & 105.31 & 95.20 & 97.00 & 98.60 & 103.50 & 102.40 \\
\hline
\end{tabular}

$\underline{\text { Salmon River below confluence with Yankee Fork (RKm 590.7) }}$

\begin{tabular}{|c|c|c|c|c|c|c|c|c|c|c|c|c|}
\hline & Auqust & September & October & November & December & January & February & March & April & May & June & July \\
\hline Average & 72.70 & 59.68 & 57.10 & 91.66 & 93.89 & ----- & 100.19 & 101.91 & 112.51 & 118.45 & 121.67 & 118.62 \\
\hline Minimum & 59.50 & 30.90 & 51.70 & 87.76 & 90.83 & ----- & 94.90 & 93.60 & 103.60 & 113.20 & 117.50 & 111.00 \\
\hline Maximum & 88.70 & 65.60 & 62.70 & 96.09 & 97.67 & ----- & 114.00 & 123.40 & 122.10 & 123.60 & 125.40 & 136.60 \\
\hline
\end{tabular}

a Data not presented due to equipment problems.

$b$ Data loss occurred when high flows washed out the monitor. 